Portland State University

PDXScholar

\title{
Modeling the Co-Production of Public Sector Innovation: Strategic Dimensions of Organizational Innovation within the Public Maritime Ports of the Pacific Northwest
}

Christopher R. Davis

Portland State University

Follow this and additional works at: https://pdxscholar.library.pdx.edu/open_access_etds

Part of the Public Affairs Commons, and the Public Policy Commons Let us know how access to this document benefits you.

\section{Recommended Citation}

Davis, Christopher R., "Modeling the Co-Production of Public Sector Innovation: Strategic Dimensions of Organizational Innovation within the Public Maritime Ports of the Pacific Northwest" (2018). Dissertations and Theses. Paper 4231.

https://doi.org/10.15760/etd.6115

This Dissertation is brought to you for free and open access. It has been accepted for inclusion in Dissertations and Theses by an authorized administrator of PDXScholar. Please contact us if we can make this document more accessible: pdxscholar@pdx.edu. 
Modeling the Co-Production of Public Sector Innovation:

Strategic Dimensions of Organizational Innovation within the Public Maritime Ports of the Pacific Northwest

by

Christopher R. Davis

A dissertation submitted in partial fulfillment of the requirements for the degree of

Doctor of Philosophy
in
Public Affairs and Policy

Dissertation Committee:

Craig Shinn, Chair

Marcus Ingle

Birol A. Yesilada

Sy Adler

Portland State University

2018 
C 2017 Christopher R. Davis 


\begin{abstract}
Innovation is vital to employing resources in times when the complexity and the demand for public goods and services strain organizational capacities. While innovation in the private sector is the subject of intense academic interest, the study of this phenomenon in the public sector pales in comparison. This is troubling because innovation is an important tool for overcoming resource limitations that plague the public sector. This dissertation's unique contribution to the field is the creation and empirical validation of a model that explains and predicts the co-production of public sector innovation. The model explains the causal mechanism of innovation and has predictive value.
\end{abstract}

No generally agreed upon or empirically tested theory exists for understanding or predicting the social interactions that lead to public sector innovation. This dissertation closes this gap by using prior research and empirical observations to build and validate a model that explains the co-production of public sector innovation at the nexus of leadership, the organization, and the customer or client of the organization. The findings, conclusions, and recommendations of this study bridge theory and practice to define the conditions that lead to co-production of public sector innovation.

This dissertation employed a deductive-inductive typological approach that used grounded theory to describe the conditions present when innovation occurs. These conditions exist as antecedents that include adaptive interest alignment, client-based prioritization, co-production readiness, organizational incentives, and organizational structure and culture. This study defined and then measured six independent variables 
that indicate the antecedents' presence. These antecedents served to predict the opening of a pathway to co-production of public sector innovation.

Empirical measurement of the six independent variables served to indicate the presence or absence of the antecedents that operate in three intersecting domains (leadership, organization, and clients or customers). The independent variables are present when the dependent variable of co-produced public sector innovation emerged. The creation of two unique indices provided an aggregate summary of the variables. The indices served as proxy measures of co-produced public sector innovation. Special districts served as the empirical setting for this research.

A case study approach served to validate the model using indices of the expected and actual measurement of co-production of innovation in the public sector. This dissertation validated the theoretical framework that served as a heuristic tool for conceptualizing the dynamics that moderate the co-production of public sector innovation within a defined political economy. The findings, conclusions, and recommendations that emerged from this research contribute to the ongoing dialogue about the conditions necessary for public sector innovation to occur. 


\section{DEDICATION}

In dedication to my wife Patti. Without her, I would not be the person that I am today. I am profoundly grateful for your love, encouragement—and occasional prodding - along our journey together. Thank you for giving me the freedom and support to chase my dreams and create a better tomorrow. 


\section{ACKNOWLEDGEMENTS}

I wish to acknowledge Dr. Craig Shinn for his unwavering support and encouragement in pursuing my own path. I wish to thank Dr. Marcus Ingle for inspiring me to undertake this dissertation. I wish to express gratitude to Dr. Birol A. Yesilada and Dr. Phillip Cooper, without their guidance and willingness to share their knowledge this dissertation would not have been possible. I would also like to thank my cohort, especially Kelly Sherbo and Andrew Dzeguze. I have profited greatly by my collaboration with Kelly on special districts. Andrew has been an inspiration, fount of knowledge, and a wonderful colleague. Finally, I wish to thank Dr. Sy Adler and the countless other individuals at Portland State University that believed in my dream and provided support along the way. 


\section{TABLE OF CONTENTS}

Abstract $\quad$ i

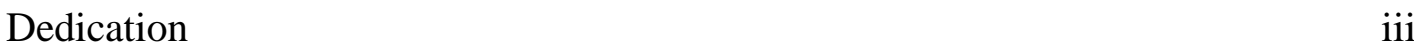

Acknowledgements iv

List of Tables $\quad$ X

List of Figures $\quad$ xii

Chapter 1: Innovation in the Public Domain $\quad 1$

$\begin{array}{ll}\text { Introduction } & 2\end{array}$

$\begin{array}{ll}\text { Background and Purpose of the Study } & 6\end{array}$

Public Versus Private Sector Innovation: Is There Really a Difference? 15

$\begin{array}{lr}\text { Problem Statement } & 19\end{array}$

$\begin{array}{ll}\text { Implied Theoretical Basis } & 22\end{array}$

$\begin{array}{ll}\text { Empirical Setting and Study Population } & 27\end{array}$

$\begin{array}{ll}\text { Research Steps } & 35\end{array}$

$\begin{array}{ll}\text { Assumptions and Limitations } & 38\end{array}$

The Road Ahead: A Summary of the Dissertation 44

$\begin{array}{ll}\text { Chapter 2: Setting the Stage } & 50\end{array}$

Introduction $\quad 52$

Innovation at the Organizational Level $\quad 58$

Summary and Synthesis of Innovation at the Organizational Level $\quad 70$

$\begin{array}{ll}\text { Measuring Public Sector Innovation } & 77\end{array}$

$\begin{array}{lr}\text { Innovation Below the Organizational Level } & 80\end{array}$ 
Innovation Above the Organizational Level 85

$\begin{array}{ll}\text { Leadership, Organizational Culture, and Innovation } & 88\end{array}$

$\begin{array}{ll}\text { Public Sector Co-production } & 94\end{array}$

$\begin{array}{ll}\text { The Origins of Special Districts } & 100\end{array}$

$\begin{array}{ll}\text { Special Districts as Empirical Setting } & 106\end{array}$

$\begin{array}{lr}\text { Conclusion } & 111\end{array}$

Chapter 3: Public Sector Organization Innovation Model 113

$\begin{array}{ll}\text { Introduction } & 114\end{array}$

$\begin{array}{lr}\text { Definition of Terms } & 118\end{array}$

$\begin{array}{lr}\text { Conceptualization } & 122\end{array}$

$\begin{array}{lr}\text { Associational Statements } & 129\end{array}$

$\begin{array}{ll}\text { Empirical Orientation } & 133\end{array}$

Public Sector Innovation Organizational Index (PSIOI) 136

Organizational Antecedents of Public Sector Innovation (OAPSI) 144

Ethical Considerations and Statement of Disclosure 155

$\begin{array}{lr}\text { Conclusion } & 160\end{array}$

Chapter 4: Operationalizing the Model 162

$\begin{array}{ll}\text { Introduction } & 162\end{array}$

$\begin{array}{ll}\text { Research Strategy } & 164\end{array}$

$\begin{array}{ll}\text { Research Approach } & 165\end{array}$

$\begin{array}{ll}\text { Research Design } & 174\end{array}$

$\begin{array}{lr}\text { Tools, Techniques, and Procedures } & 180\end{array}$

$\begin{array}{ll}\text { Data Collection and Instrumentation } & 184\end{array}$ 
Public Sector Innovation Organizational Index (PSIOI) Formulation

Organizational Antecedents of Public Sector Innovation

(OAPSI) Formation

Case Study Organization and Layout

Conclusion

Chapter 5: Case Study Analysis and Presentation

Introduction

Case 1: Life Sciences Facility

Background and Setting

Public Sector Innovation Organizational Index (PSIOI)

Organizational Antecedents of Public Sector Innovation (OAPSI) 218

Leadership-Client Co-Production

Client-Organization Co-Production

Organization-Leader Co-Production

Within-case Analysis

Case 2: Creating Intergovernmental Capacity

Public Sector Innovation Organizational Index (PSIOI)

Organizational Antecedents of Public Sector Innovation (OAPSI) 
Case 3: Reconciling Conflicting Demands among Customers and Clients 257

$\begin{array}{ll}\text { Background and Setting } & 257\end{array}$

Public Sector Innovation Organizational Index (PSIOI) 259

Organizational Antecedents of Public Sector Innovation (OAPSI) 262

Leadership-Client Co-Production 262

Client-Organization Co-Production 265

Organization-Leader Co-Production 269

$\begin{array}{ll}\text { Within-case Analysis } & 273\end{array}$

Between-Case or Cross-Case Analysis 276

Leader-Client Co-Production Domain $\quad 279$

Client-organization Co-Production Domain 281

Organizational-Leader Co-Production Doman 286

$\begin{array}{ll}\text { Conclusion } & 293\end{array}$

$\begin{array}{ll}\text { Chapter 6: Conclusion } & 295\end{array}$

$\begin{array}{ll}\text { Introduction } & 296\end{array}$

$\begin{array}{ll}\text { Analytical Approach } & 299\end{array}$

Data Collection and Analysis Methodology 303

Findings, Conclusions, and Recommendations 309

Finding 1: Adaptive Interest Alignment 314

Finding 2: Client-based Prioritization 318

Finding 3: Co-Production Readiness 324

Finding 4: Organizational Structure and Culture 330

Finding 5: Organizational Incentives 335 
Summary of Findings and a Revision of the Model 345

Value of Study to Practice, Theory, and Public Policy 348

Future Research Opportunities 356

$\begin{array}{ll}\text { References } & 361\end{array}$

Appendix A: Data Collection Instrument (Interview Guide) 380

Appendix B: Case Content Analysis Coding Book (Codebook) 384 


\section{LIST OF TABLES}

Table 1. Special District Functional Classifications 108

Table 2. Definition of Key Terms 122

Table 3. Test of Implied Model Validity 132

$\begin{array}{ll}\text { Table 4. Definition of PSIOI Terms } & 143\end{array}$

Table 5. Organizational Antecedents of Public Sector Innovation (OAPSI) 152

Table 6. Comparisons of Methodological Research Approaches 170

Table 7. Unit of Analysis Typology in the Case Study Approach 176

Table 8. Case 1: Public Sector Innovation Organizational Index 231

Table 9. Case 1: Organizational Antecedents of Public Sector Innovation 233

Table 10. Case 2: Public Sector Innovation Organizational Index 254

Table 11. Case 2: Organizational Antecedents of Public Sector Innovation 256

Table 12. Case 3: Public Sector Innovation Organizational Index 274

Table 13. Case 3: Organizational Antecedents of Public Sector Innovation 275

Table 14. PSIOI Leader-Client Co-Production Cross-Case Analysis 279

Table 15. OAPSI Leader-Client Co-Production Cross-Case Analysis 280

Table 16. PSIOI Client-Organization Co-Production Cross-Case Analysis 282

Table 17. OAPSI Client-Organization Co-Production Cross-Case Analysis 283

Table 18. PSIOI Organization-Leader Co-Production Cross-Case Analysis $\quad 287$

Table 19. OAPSI Organization-Leader Co-Production Cross-Case Analysis $\quad 288$

Table 20. Study Findings, Conclusions, and Recommendations 313

Table 21. Revised Organizational Antecedents of Public Sector Innovation 345 
Table 22. Emergent Themes and/or Topical Insights 


\section{LIST OF FIGURES}

Figure 1. Strategic Dimensions of Public Sector Organizational Innovation Model 10

Figure 2. Dissertation Research Steps 36

Figure 3. Leader-Client Co-Production Domain 148

Figure 4. Client-Organization Co-Production Domain 149

Figure 5. Organization-Leader Co-Production Domain 150

Figure 6. Philosophy of the Social Sciences Research Design Paradigm 166

Figure 7. Case study Approach Research Design 175

$\begin{array}{lr}\text { Figure 8. Comparative Case Selection Criteria } & 182\end{array}$

Figure 9. Method of PSIOI/OAPSI Comparative Analysis 184

Figure 10. Dissertation Data Collection Methodology 188

$\begin{array}{ll}\text { Figure 11. PSIOI Case Selection Methodology } & 197\end{array}$

Figure 12. Revised Strategic Dimensions of PSOI Model 348 


\section{CHAPTER 1: INNOVATION IN THE PUBLIC SECTOR}

To hear John Micklethwait and Adrian Woodridge (2014) tell it, public sector governance is the reason that the Western world has achieved unparalleled economic, social, and political success in the twenty-first century. In their view, the emergence of the modern liberal nation-state prevailed because it provided the conditions necessary for social harmony, political stability, and economic prosperity. The modern liberal nationstate accomplished this by bringing order and efficiency to the chaos of a Hobbesian world in the late sixteenth century, and then fostering the liberty and freedom necessary for prosperity in the eighteenth century.

The result was the emergence of the modern welfare state in the twentieth century. This would not have been possible, contend Micklethwait and Woodridge (2014), without public governance. Moreover, not just any governance, but good public governance. Meritocracy, accountability, and scientific thinking are the basis of such a government. According to their line of reasoning, if you wish to improve the human condition all you need is a state capable of the production and implementation of the best possible ideas.

Micklethwait and Woodridge (2014) go on to state that the success of contemporary Western governance is now threatened. From their viewpoint unsustainable public debt, coupled with an explosion in information technology, and the emergence of alternate governance paradigms - in particular the authoritarian models of China and Singapore - are leading to a revolution in modern public governance. Added to this mix 
is an increasing demand by constituents and clients for public goods and services (Micheli et al. 2012)

Micklethwait and Woodridge (2014) are not alone in suggesting that obsolete forms of public governance are inadequate to address the task of creating a better tomorrow (Dror 2001). Micheli et al. (2012) assert, moreover, that resistance to change, risk aversion, and hierarchical structures act as barriers to improving public governance. What is at stake, Micklethwait and Woodridge (2014) assert, is nothing less than which form of public governance will prevail in a globalized world. They contend that the worldwide race to reinvent the state is now underway.

\section{Introduction}

The reinvention and reconstruction of public governance systems is nothing new. As already noted the structure of public governance has undergone reinvention and reconstruction since well before the time of Hobbes. In this regard, the evolution of pubic governance is happening in much the way that it always has. While the dust has hardly settled, it is nevertheless clear that innovation will play a necessary role. This perspective suggests that one of the defining characteristics of the winning system of public governance is the capability to implement innovations that result in marginally better results than do their competitive counterparts.

The innovations of which Dror (2001), Drucker (1998), and Micklethwait and Woodridge (2014) write spring from the institutional routines imbedded in governing organizations. In this context, organizational outcomes are the artifacts of innovation. Organizational innovation represents an adaptive mechanism. Through it, organizations 
increase their chances of survival in their respective strategic environments. Micheli et al. (2012) assert that recent initiatives by public sector organizations to adopt new business models that leverage public-private partnerships reflect this perspective.

Drucker (1998) posits that innovation is both scalable and critical to organizational, national, and regional success. He goes on to propose that innovation emerges though the combination of two or more fairly well known things in new and unique ways. In the context of the public sector, these can be new ideas, processes, policies, procedures, technology, or services (Edquist 2001). Innovation also requires a culture capable of absorbing new and surprising things in a familiar and anticipatory manner (Morgan 2006).

Innovation in the public sector is imperative for good governance and the achievement of the common good. The unit of analysis for good governance ties to the organizational level while the unit of analysis for the common good links to the societal level. In this context, the values of a society shape the political economy. These values, in turn, embed within societies institutions. The political economy shapes the strategic environment that, in turn, conditions the public sector organization, its leaders, and their customers and clients. Innovation in this regard is an instrument of good governance and serves the common good.

Innovation, like good governance, is normative in nature. Innovation represents a metaphorical empty vessel the contents of which are the result of the dynamic interplay between the organization, its leaders, and their customer and clients within a contextual strategic environment. In this respect, the values that leaders bring to the practice of governance and the norms that predominant in the organization and its strategic 
environment imbue. This suggests that innovation serves, therefore, as one-albeit one of the more important- - tools of good governance.

This dissertation seeks to frame the theoretical and practical relationship between public sector innovation, leadership, the organization, and its customers or clients. These relationships, moreover, are imbed in a strategic environment that places constraints and restraints on the actions of both institutions and individuals. This dissertation advances a theoretical framework that describes and explains the co-production of public sector innovation. The findings, conclusions, and recommendations that emerge from this study will contribute to the ongoing dialogue about what is necessary for public sector innovation to occur.

The concept of co-production provides a theoretical framework for defining the relationship between the economy and society. Olson (1965) posits that in the absence of coercion the provision of common public goods and services becomes increasingly suboptimal as the size of group increases. Ostrom (1996) counters this orthodoxy. She argues that community, and not competition, is at the core of providing public goods and services. Co-production describes the process or mechanism by which individuals and organizations mobilize to cooperate in the production of public goods and services.

The co-production of public sector innovation is a necessary avenue for improving social outcomes (Micheli et al. 2012). It springs from a normative base. This dissertation offers a holistic model with descriptive and explanatory powers. The theoretic framework this dissertation puts at risk describes and explains the causal pathway to the co-production of public sector innovation. The objective of this study is to 
provide practitioners of public sector governance insight into the theory and practice of co-producing innovation.

Chapter 1 provides some background on why the study of public sector innovation is so important. The intent in doing so is to provide an answer to the "so what" and "as compared to what" questions. The next section of this chapter provides some background on the subject of co-producing public sector innovation, serving to frame this complex phenomenon and place it in perspective. A careful review of the literature on public sector innovation reveals that what is lacking is a generally agreed-upon empirical model that explains public sector innovation. The next section of this chapter introduces a model that fills this gap, providing an analytically robust explanation of the causes of coproduction of innovation in the public sector.

The section that follows explores various perspectives on public and private sector innovation. In doing so, it places in perspective the model that this dissertation puts at risk. The fourth section presents the problem this dissertation seeks to solve. This dissertation will close the gap in our understanding of public sector innovation using the case studies. The section that follows presents the implied theoretical basis for this dissertation. The next section defines the empirical setting and study population of this inquiry.

Special districts represent a little-studied but critically important species of public governance. They are independent governmental units that exist as separate entities with substantial administrative and fiscal independence. They represent the study population of this dissertation. This chapter also includes a section that presents the research steps in this empirical setting. The eight section addresses some of the assumptions and 
limitations inherent in this study. The final section provides a brief summary of this dissertation.

\section{Background and Purpose of the Study}

Sorensen and Torfing (2012) conclude that "the dearth of studies focusing on... innovation in the public sector is not least regrettable in light of the increasing demand for public sector innovation" (844). This is, consequently, an area of particular interest to today's public sector theorists and practitioners (Walker 2008). Today the complexity and demand for public goods and services strains organizational capacities. This is the case on a fiscal and institutional level. Rising economic inequity also serves to create increased demand. The need for innovation is rooted in the strategic environment.

While innovation in the private sector is the subject of intense academic interest, the study of this phenomenon in the public sector pales in comparison (Shin and McClomb 1998). This is especially troubling because innovation is an important avenue for overcoming resource limitation in the public sector. Governments today face an extraordinary range of challenges from shifting demographics, increasing populism, transforming economic structures, and changing attitudes towards public policy (Salamon 2003). Innovation is vital to employing resources in a time when the complexity and the demand for public goods and services strains organizational capacities.

Today's challenges demand that public sector organizations nurture innovation. Innovation, in the best of times, is often difficult, challenging, and fraught with risk to the individual and the organization. A commitment to change and cognitive flexibility is a prerequisite (West and Farr 1990). Additionally, an organization's leadership plays a 
central role in fostering innovation and change (Vasccaro et al. 2012). Likewise, the organization itself can either resist or be an impetus for innovation and change (Zimmermann 1999).

It is possible to observe innovation at the individual, team, organizational, institutional, and even societal levels (Porter 1990; Van de Ven, Angle, and Pool 1989a). At each of these levels, it is the subject of definition and study through empirical observation. This dissertation seeks, however, to explore public sector innovation at the organizational level. The unit of analysis for this dissertation is the individual engaged in organizationally appropriate relationships that result in the co-production of innovation in the public sector.

Mintzberg (1983) advises that organizations exist to the extent that individuals cooperate in completing a task. He goes on to suggest that this requires both the division and coordination of labor among its members. Scott (2003) expands upon this rationalist approach to propose that organizations exist in terms of roles and relationships. Mintzberg (1983) and Scott (2003) are describing largely hierarchical relationships that exist within an institutional setting. The empirical setting for this study is special districts and they are typically small to medium sized organizations.

While work of earlier rational theorist like Zaltman, Duncan, and Holbek (1973) and Van de Ven and Poole (1989) provides a useful backdrop for understanding the complexity and interrelated nature of innovation, it is the later theorists - in particular, Walker (2010) and Bason (2010) — that provide the best point of departure. Subsequent chapters will consider the conceptualization of public sector innovation in detail. In this dissertation, the definition of the co-production of public sector innovation is systemic 
behavioral change that results from practices imbedded in institutions that both

outperform previous practices and result in the common good.

The topic of this study is the creation and validation of a model that explains the co-production of public sector innovation. In other words, this dissertation is an exercise in theory creation. Co-production serves to expand organizational boundaries. It does so by including as active participants in the process of innovation the customers or clients of the organization (Ostrom 1996). Co-production, in this framework, is a form of political economy. Ostrom (1996) posits that co-production results in the joint creation of a mutually valued outcome.

Co-production involves the equal and reciprocal cooperation in the creation of public goods and services. This occurs in the context of a relationship between those professionals delivering public goods and services and those individuals consuming the goods and services. In this dissertation, these individuals are the organizations customers or clients. Co-production exists, furthermore, for making better use of each other's assets and resources (Ostrom 1996). The underlying assumption is that this will result in improved efficiency and effectiveness in achieving positive social outcomes.

Early theories of innovation within organizations depicted it as a linear process involving rational decision-making (Weber 1964). From this perspective, structural variables affect the outcome of organizational activities. According to this viewpoint, organic and mechanistic structures have an important impact on innovation (Burns and Stocker 1961). Constructing a social theory by focusing on the development of a sociological view of institutions dominated early approaches to studying innovation. This 
form of institutionalism focused on how individuals interact in a social setting and the effect that institutions have on society.

Zaltman, Duncan, and Holbek (1973) define "innovation" as "any idea, practice, or material artifact perceived to be new by the relevant unit of adoption" (158). They assert that "innovation" differs from "change" in that innovation represents an alteration of the structure or functioning of an existing social system and, therefore, causes change. Only the perception of new change, however, results in innovation according to their theory of the phenomenon. Their working definition of innovation serves as the jumpingoff point for this dissertation.

The dominant tendency in the study of innovation is to employ qualitative methodologies, such as case studies and surveys, to infer particularistic theories which are fragmented and inconclusive in explaining the observed phenomenon (Van de Ven, Harold, and Poole 1989a; Walker 2008; Bason 2010). Indeed no generally agreed-upon and empirically tested theory of innovation in the public sector currently exists. This suggests the need for the development and operationalization of a flexible and adaptive theoretical framework that explains innovation in the public sector. The critical first step is defining a theoretical construct for the co-production of organizational innovation at the nexus of the organization, its leadership, and its customers or clients.

This dissertation proposes an integrated model that describes the co-production of organizational innovation in the public sector. The model also provides an explanation of this systemic phenomenon. The intent is to provide an archetypal framework for understanding the co-production of public sector innovation. Figure 1 depicts the strategic dimensions of public sector organizational innovation model. This framework 
posits that the actions of three principle actors - the organizational leader, the organization itself, and the organization's customer or client-are central to the coproduction of organizational innovation.

Figure 1. Strategic Dimensions of Public Sector Organizational Innovation Model

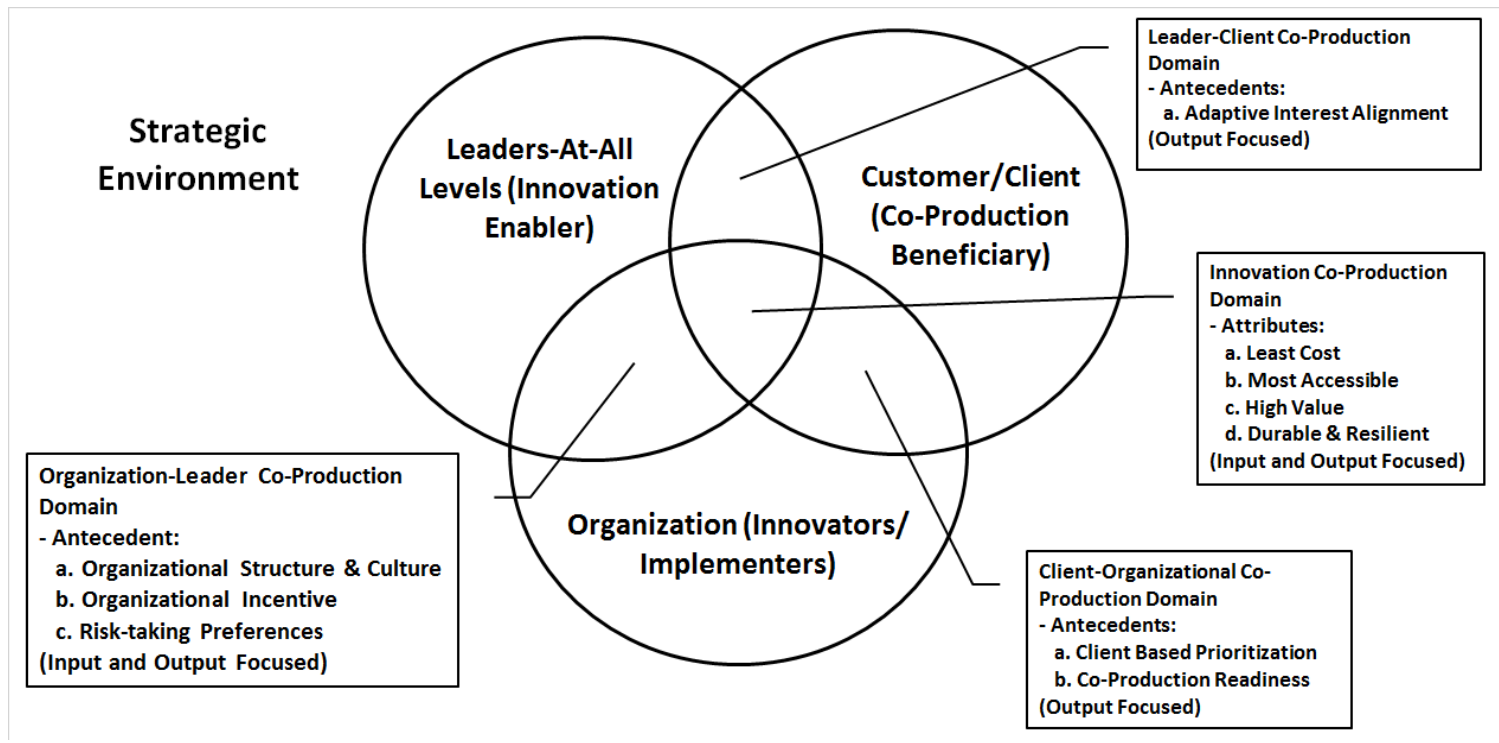

While each actor shares unique domains in relation to themselves and other actors, all three share the domain of innovation. These principle actors, however, exist within a strategic environment. This strategic environment shapes the actions of individuals and organizations. In addition, individuals and organizations shape the strategic environment. In this manner, the creation of the strategic environment occurs through an iterative process (Rainey 1997). The strategic environment reflects the context in which individuals act with a knowledgeable expectation of risk and reward for themselves and their respective organization. 
In the context of this dissertation "leadership" manifests as the institutionally embedded and situationally appropriate practice of creating relationships for the purposeful achievement of the public good (Shove, Pantzar, and Watson 2012; Goeman, Boyatzis, and McKee 2002). In this regard, leadership can appear at any level of the organization depending on institutional conditions. The term "customers and clients" used in this dissertation refers to individuals in relationships for the purposeful achievement of the public good. Subsequent chapters deal with the conceptualization of leadership, customers, and clients in more detail.

It is important to note that customers and clients differ from citizens. The latter reside in the jurisdiction of a government, such as a special district, and possess the ability to impose accountability by democratically sanctioning the special district (Manin, Przeworski, and Stokes 1999). This may or may not be the case with customers or clients. It is possible, however, for customers or clients to influence special districts through market mechanisms. Special districts by their very nature, however, can produce both public and private goods (Morgan et al. 2013).

Van Acker and Bouckaert (2017) argue, "Issues of power and politics should receive equal attention in the study of public sector innovations" (17). While public sector organizations are public regarding, the customers and clients of these organizations are private or self-regarding. Special districts, because they tend to focus on delivering a single or small set of public good or services, are inclined to provide benefits that are concentrated while the costs are diffuse or partially borne by the customer or client. The concentration of benefits serves to create incentives for a narrow band of customer or clients to maintain the good or service in perpetuity. 
Organizations provide the institutional mechanism for collective action (Olson 1971). Organizations nominally exist for a particular purpose and are composed of formal and informal structures (Mintzberg 1983). Organizations are ubiquitous. They exist as manifestations of the near-decomposability means of securing the benefits of coordination while holding down the costs by an appropriate division of labor among sub-units (Simon 2000). One focus of this dissertation is on the organization and its role in the co-production of public sector innovation.

In the framework of this dissertation, the strategic environment represents culturally cognitive patterns and normative codes of thought and behavior that exhibit rule-like regularity on individuals and institutions (Scott 2003). The strategic environment largely reflects the prevailing political economy. It is a manifestation of a complex and dynamic milieu in which a wide range of social factors continually interact (Morgan 2006). As such, assessing the strategic environment and making a correct prognosis are some of the most difficult tasks in the field of public policy.

The social factors that animate the model include, for example, the legal regime. Laws represent artifacts of the values imbedded within institutions at their founding (Weber 1964). Demographic differences also shape the social dialogue, while technology often defines the limits of social achievement (Morgan 2006). Evolving economic structures modulate individual and institutional behavior in new and different ways (Galbraith 1998). The social and cultural context in which the political economy imbeds also molds it in unique ways (Wallerstein 1974).

The goal of this research agenda is to advance a practical framework that describes and explains public sector innovation for the practitioner and academic alike. 
This framework will provide a tool for pursuing and fostering innovation within public sector organizations. This dissertation proposes an organic, heuristic model that recommends situationally dependent best practices. It is, by design, not mechanistic in nature. In other words, the model requires judgement in application. It is also contextual in nature.

In seeking to achieve this goal, it is important to understand what the coproduction of innovation looks like within the space carved out for innovation at the nexus of leadership, the organization, and the customer or client. Co-production must occur while acknowledging the salience of environmental factors. Therefore, it is important to take into consideration the general and specific environmental conditions in which the organization finds itself (Rainey 1997). Environmental conditions are those factors that influence the organization such as the political economy.

The model suggests certain antecedents are present when innovation occurs. This study defines and measures ten independent variables that indicate the antecedents are present when the dependent variable of public sector innovation emerges. Antecedents are those conditions that manifest in the presence of innovation and provide the causal pathway for innovation. The purpose of this study is to validate the model. Confirming that the antecedents suggested by the model are operative when public sector innovation occurs validates model. Operationalizing the concept of innovation occurs through the measurement of variables that suggest the presence of the antecedents. Subsequent chapters define each variable and frame its relationship to the antecedent.

The power of this model is its ability to provide a descriptive and causal explanation of the phenomenon. Operationalization of the model requires the 
establishment and empirical measurement of variables. Subsequent chapters outline the manner in which it is possible to ascribe value to these variables. The ordinal composite scale employed to measure the variables uses the number, significance, robustness, and direction of each to assign a value. The subsequent aggregation of the measures serves to answer the question of how innovative is a public sector organization in theory and practice.

Operationalizing the model by identifying and measuring independent variables associated with the antecedents will allow for a causal understanding of the dependent variable of innovation in the public sector. Antecedents create the conditions that exist in the presence of innovation. It is possible, moreover, to observe the variables associated with the antecedents. As such, the variables are subject to empirical measurement. The independent variables that the model suggests serve as indicator variables of the antecedents include, for example, the existence of feedback loops, leading with a vision, ability to experiment, and incentives for improvement activities. The underlying assumption is that the higher the organization ranks on the aggregate summaries of the independent variables, the more innovative the organization.

This dissertation employs the case study approach to validate the model. Since this is an exercise in theory creation, only those cases in which innovation occurred were selected (Yin 1994). In other words, the presence of the dependent variable of innovation was the criteria for case selection. This study documents the development and employment of a composite index for measuring the degree of innovation expected in an organization. Public sector innovation organizational index (PSIOI) serves to represent 
the concept of expected innovation. The creation of the index involved coding available documents.

This PSIOI provides an indication of expected innovation. This served as the basis for case selection. An organizational antecedent of public sector innovation (OAPSI) rating constructed from empirical observations provided a measure of actual innovation. For each case with a positive PSIOI an OAPSI rating was developed. Field interviews provided empirical observations of the independent variables. The empirical measurement of the variables served to indicate the presence of the antecedents. The antecedents created the pathway to the co-production of public sector innovation. Through the empirical observation of this process, it was possible to validate the model. The model, therefore, provided an explanation of the causal mechanisms of innovation that has predictive value.

\section{Public Versus Private Sector Innovation: Is There Really a Difference?}

There is an unsettled debate regard the nature and extent of the difference between innovation in the public and private sectors (Rainey 1997; Sorensen, and Torfing 2011; Hartley et al. 2013). This debate flows chiefly from differing perspectives on the influence of the strategic environment on public and private sector organizations. How this debate plays out has enormous implications for the study of innovation. Chief among these implications is the ability of theoretical constructs to span the sectors and still maintain currency.

It is possible that the drivers and barriers to innovation are so exclusive to each sector that two uniquely separate theories are necessary to understand the phenomenon of 
innovation. While it is beyond this dissertation to recommend an answer to this question, it is, nevertheless, beneficial to take note of the drivers and barriers to innovation that exists, regardless of the sector. This is because any theory on innovation at the organizational level must take into account the impact of differing environmental factors (Morgan 2006).

At first glance, there are many easily observable environmental differences between the public and private sectors. The most salient of which is the profit motive (Sorensen and Torfing 2011). A number of theorists contend that the private sector is largely profit-driven (Porter 1990; Hartley 2005; Sorensen and Torfing 2011). The public sector relies on the market to condition organizational behavior and adjudicate completing values. Public organizations have, alternatively, multiple and often competing socio-political drivers.

It is the political process, conversely, which governs the behavior of public sector organizations. Values arbitration is through political institutions. One view holds that the absence of market forces creates a significant barrier to public sector innovation (Hartley 2005). This stems from the perspective that economic competition is the primary motivator of innovation (Porter 1990). Absent economic competition, according to this view, there is little impetus for innovation. Lack of a profit motive, therefore, equates to an absence of innovation.

Another difference between the public and private sector is the type of goods and services the organization focuses on delivering (Olson 1971). The prevailing view is that private sector firms mainly focus on exclusive or private goods and services (Hartley et al. 2013). They are exclusive to the extent that it is possible to restrict or limit their 
benefits to a particular individual or organization (Olson 1971). The effectiveness of these products in meeting economic needs largely defines organizational success in the private sector.

The delivery of public or inclusive goods and services dominants the activities of public sector organizations. The inclusive nature of the goods and services derives from the fact that an individual's consumption of the goods and services does not affect another's consumption. The opposite is also true. An example is national defense and public parks — where all citizens' benefit from the service provided regardless of whether they contribute. The focus in the public sector milieu, moreover, is on the efficiency and equity of the delivery process (Morgan 2006). There is, in other words, a normative aspect to the production of public sector goods and services.

There is also a belief that public and private sector organizations differ in their risk preferences. One view is that public sector organizations are more risk adverse (Hartley et al. 2013). This is the case for two reason. The first is that these organizations are under the guidance of risk-adverse politicians. The second reason is that any failure is sure to evoke public scrutiny and condemnation (Micheli et al. 2012). This is especially the case when members of society perceive their individual contributions are being lost or squandered. There is, moreover, a perception that the private sector is the domain of risktaking entrepreneurs. From this perspective, it is possible to argue that not only do business entrepreneurs tolerate risk more than in the public sector but also that failures generally receive little in the way of examination and condemnation.

There are a number of theorists, conversely, who contend that the drivers and barriers to innovation in the public and private sectors are similar (Van de Ven 1986; 
Sorensen and Torfing 2011; Hartley et al. 2013). This viewpoint reflects the perspective that organizations inhabit an environment in which their survival depends on their ability to respond to a wide variety of sanctions and incentives. The individuals that inhabit public and private sector organizations, moreover, similarly react to environmental stimulus. The need for personal growth, enhanced professional reputation, or the security of belonging are just as salient drivers of individual behaviors as is profits (Morgan 2006).

Hartley, Sorensen, and Torfing (2013) conclude that public and private sector firms face many of the same organizational and bureaucratic challenges. Their contention is that the domain of innovation involves either open co-production or closed-ended inhouse efforts - regardless of the sector. Powerful and influential interests are just as likely to inhabit the pubic as the private sector according to this perspective. The result is the inevitable contestation of goals. This view holds that any difference between the public and private sector is largely superficial. Hartley, Sorensen, and Torfing (2013) conclude there are few differences in the drivers and barriers that organization face in the public and private sectors.

Special districts add to the contested nature of the pubic-private debate over innovation. The empirical setting for this dissertation is one such special district. In this regard, public marine ports provide their customers and clients access to facilities and services that are a source of economic benefit. Often these facilities represent exclusive goods. In other words, the customer or client of the port that is using the port's facilities has sole access to its benefits (Olson 1971). The result is that pubic ports have one proverbial foot in the public sector and one in the private sector. In studying innovation in 
special districts, therefore, this dissertation adds additional perspective to the publicprivate debate.

Public sector innovation reflects systemic behavioral change that results from practices imbedded in institutions that both outperform previous practices and results in the common good. This reflects the normative aspect of public sector innovation and distinguishes it from the private sector variety. This is not to suggest that private sector innovation is devoid of social benefit. Indeed, market mechanisms can, and do, produce such benefits (Salamon 2003). This is the case when there exists an alignment between the goals of the individual or organization and those of the larger society. The normative aspect of public sector innovation is, instead, a recognition that these organizations "operate under political authority and without economic markets for their outputs" (Rainey 1997, 87).

\section{Problem Statement}

The purpose of this dissertation is to develop an applied theoretical framework for understanding and explaining the co-production of public sector innovation at the nexus of leadership, the organization, and the customer or client of the organization. This model suggests that certain antecedents are present when innovation occurs in the public sector. Leaders at all levels of an organization can use this knowledge to foster these antecedents in the expectation of innovation. This framework reflects a model or theory that represents a closed system of processes and outcomes from which it is possible to make a prediction of the nature of the social world and that is open to some kind of empirical test (Dubin 1969). 
Co-production embeds, moreover, within a unique social and political milieu. This milieu is itself the result of political, social, and economic traditions. Constitutional provisions and their codification within legislative, judicial, and social institutions reflect in the existing political economy (Rainey 1997). Social, demographic, political, and technological influences in the strategic environment likewise serve to contextualize coproduction. Any theory or model of public sector innovation, therefore, must take into account the strategic environment.

Previous studies of innovation in the public sector provide the basis for the theoretical framework this study puts at risk. This theoretical framework describes and explains how leadership, the organization, and the customer or client of public sector organizations interact to co-produce innovation within a distinct political economy. This study assumes, as a starting point, that improving the delivery of public goods and services has a social utility. This can only occur if there is an institutionalized process for arbitrating self-interested values when they come into conflict with the common public interest.

If there are no value conflicts then markets are capable of efficiently and effectively delivering public goods and services. Market mechanisms, however, are incapable of arbitrating value disputes and thus political conflict often results from market failures (Morgan et al. 2013). All this implies, furthermore, the existence of legitimizing democratic norms such as an idea that those who bear the consequences of a decision have a say in that decision (Dahl 1956). This basic democratic norm reflects the integral role that individuals play in legitimizing sovereignty. 
This study provides worthwhile insights into the theory and practice of innovation in the public sector. This benefits both practitioners and theorists alike. The practitioner gains a perspective that enables public sector organizations to engage effectively and efficiently in activities leading to the co-production of innovation. The theorist will gain additional insight upon which to advance the study of public sector innovation. This dissertation builds on the existing literature and suggests opportunities for future research.

Special-purpose governments or special districts are independent government entities (Burns 1994). They exist separate and apart from general-purpose governments (Morgan et al. 2013). The definition of school districts excludes school districts while general-purpose government includes county and municipal governments (Foster 1997). Both general-purpose governments and special districts are creatures of state government. They have elected or politically appointed governing boards and use public funds to accomplish tasks allowed under state legislation.

The literature indicates that few studies on organizational innovation within special districts exist. Special districts, moreover, continue to proliferate and have grown at rates that surpass general-purpose governments (Burns 1994). The specific empirical setting in which this study is embedded is special districts, and specifically public marine port authorities. These governmental authorities represent one cross-section of special districts-albeit an important one. In the Pacific Northwest, public marine ports exist to foster economic development in the communities in which they operate. This provides an impetus to innovate since innovation provides one potential avenue for fostering economic development within the community. 
The strategic environment sets the stage for the co-production of innovation (Ostrom 1990). The term strategic environment refers to that composite of general and specific conditions, situations, and circumstances that influence the organization. A wide variety of factors influences the strategic environment. This study does not assume a fixed or static strategic environment in which public sector organizations operate. Relaxing the assumption of a fixed strategic environment allows for the reality that innovation is a contextually dependent phenomenon.

The strategic environment is volatile, uncertain, complex, and ambiguous (Stiehm and Townsend 2002). These elements create the context in which organizations contend with their past, current and future states. This occurs through the dynamic interplay of social, political, economic, and legal factors (Morgan 2006; Scott 2003). I assert that while the strategic environment serves to modulate individual and institutional behavior the behavior of individuals and institutions can also serve to shape the strategic environment (Morgan 2006). The strategic environment is the single most influential exogenous factor effecting an organization.

\section{Implied Theoretical Basis}

Antecedents of innovation embed in leadership, the organization, and their customers or clients. This section defines each within the context of organizational innovation in the public sector. In addition, it considers the strategic environment from a political economy perspective. The intent is to establish the context for the dynamic interplay this model suggests. This implied theoretical base serves as the foundation for the model and is the cornerstone of this research agenda. The aim of this dissertation is to 
provide a heuristic tool for conceptualizing the dynamics that moderate the co-production of public sector innovation within a defined political economy.

Social and economic change outpaces the ability of governing structures to adapt (Huntington 1968). Governing organizations must innovate in an effort to respond to change and address emergent issues. This dissertation adopts the perspective that innovation and change are essential in the public sector since gaps inevitably emerge in the form and extent of public governance (Micheli et al. 2012). It is possible, however, that conditions emerge which challenge this basic assertion. This could raise doubt over the efficacy of this model. The proposed study design and research methodology should uncover evidence that counters the model if this is the case.

Leadership in this context is the capacity to animate other actors towards the creation and sustainment of organizational innovation. This includes, for example, the ability to mobilize the leader's organization and engage customers or clients. In this regard, leadership is unrelated to an individual's formal role within the organization (Micheli et al. 2012). In other words, leadership emerges from multiple levels within the organization. This suggests that the power to innovate can originate from diffuse sources. It also implies that innovation is highly context-dependent (Pfeffer 1981).

Innovation can emerge, for example, from the capability of the leader to structure factors that define the innovation space. This occurs through the creation of interpersonal alliances, networks, and informal organizations (Morgan 2006). In contrast with their private sector counterparts, leaders in the public sector face unique challenges. Therefore, for example, they must adopt a comprehensive perspective that takes into account the complex social, political, and economic milieu in which they must operate (Rainey 
1997). This includes addressing issues of social equity and political accountability. In contrast, private sector leaders can choose to externalize or ignore key aspects of their environment (Cooper 2003).

The organization represents the essential capacity for innovation. It embodies that collection of individual talents and the collective resources necessary for innovation. This suggests that organizations represent the human, financial, technological or decisionmaking means necessary to achieve the ends of innovation. While this model makes no attempt to fix the specific image of the organization it, nonetheless, acknowledge that it is "based on implicit images or metaphors that lead us to see, understand, and manage organizations in distinctive yet particular partial ways" (Morgan 2006, 4).

The model posits the elasticity of organizational boundaries. In other words, they are not fixed but are instead transitory and permeable. This suggests that boundaries can expand to include organizational subgroups necessary to maintain the space for innovation. Implied in this model is the existence of normative principles that are indigenous to the organization. Latent values embed in organizational subroutines and structures. Prior learning also conditions organizations (Levitt and March 1988). In this regard, normative values are operative under conditions of innovation.

Customers or clients often serve to frame the conditions for innovation and change within the model. These agents serve to signal the existence of gaps between existing socio-economic conditions and the central governing capacity of the public sector organization. This suggests that the existence of such a disparity results in a dynamic tension. The model posits that the emergence of this tension contributes to the 
creation of the space necessary for innovation. The existence of feedback loops ensures that information symmetry exists around the gaps.

The existence of information asymmetry, furthermore, creates conditions for the active involvement of customers or clients. The extent of the information asymmetry, therefore, would appear to play a role in innovation. Therefore, for example, the greater the asymmetry favors the customers or clients, the larger their expected involvement and likely influence on the outcome. The model suggests that while customers or clients are necessary to the process of co-producing innovation they are, nevertheless, not sufficient to trigger innovation in and of themselves.

The strategic environment is the domain of the complex and often-intractable problems faced by today's public sector organizations. This model conceives of the strategic environment as conditioning the responses of the various actors. This is not to suggest that the strategic environment controls the actions of the protagonists; however, it does exert a strong influence. The model posits the result is a simultaneous constraint and restraint on available courses of action. This is especially the case in the public sector where, unlike the private sector, it is not possible to ignore or externalize environmental factors (Cooper 2003; Rainey 1997). It is, therefore, critical to consider the range of social costs and benefits attributable to innovation.

This dissertation assumes an institutional perspective when approaching the subject of public sector innovation. March and Olsen (2009) posit that an institutional framework of rules, norms, and knowledge modulates and regulates the behavior of individuals within organizations. Mintzberg (1983) asserts that organizations institutionalize their structure along the lines of a limited and contextually based 
repertoire of configurations. This is the case in the private sector where markets function as institutions in which rules, norms, and routinized practices serve to regulate competition between market participants (Hartley et al. 2013).

Institutions represent the comprehensible and routinized processes that drive the behavior of individuals. Mintzberg (1983) holds that "organizations formalize behavior to reduce its variability, and ultimately to predict and control it" (34). This results in either continuity or change. It also serves to create the elements of order and predictability. An institution shapes individual behavior at the same time that individual action and other institutions influence an institution (March and Olsen 2009). In this context, identification and habituation are fundamental mechanisms for shaping behavior. This social behavior, moreover, is subject to theoretical modeling in a manner that allows for understanding human interactions and predicting the outcomes of these interactions (Dubin 1969).

This dissertation uses a comparative case study approach to investigate this phenomenon. Yin (1994) defines a case study as "an empirical inquiry that investigates a contemporary phenomenon within its real-life context, especially when the boundaries between phenomenon and context are not clearly evident" (13). Thomas (2011) posits that the holistic and interpretive nature of the case-oriented approach is well suited for theory building. Yin (1994) asserts that it is possible to validate a theory by logically linking data from two or more cases to a theoretical proposition.

Ragin (1987) advises that the holistic and interpretative nature of the case study approach makes it particularly well suited to theory building. It is possible to consider multiple cases as one would consider multiple experiments (Yin 1994). It is replication, 
and not sampling logic, that proves the theory. This replication is possible though comparative case analysis that aggregates and synthesizes results across a set of individual cases (Yin 1994). Theoretical replication is possible, therefore, through the contrasting of results between cases. This dissertation adheres to the recommendations of Ragin (1987) and Yin (1994) in applying the case study method to validate the theoretical model through empirical observation.

\section{Empirical Setting and Study Population}

Special districts, specifically public maritime ports, are the empirical setting for this dissertation. Foster (1997) points out that while the formation of local governments has exploded during the twentieth century the growth has come primarily from specialpurpose governments. Burns (1994) conceives of local governments as bundles of particular types of institutions that states allow and the federal government conditions. These institutional arrangements include, for example, control over service provision, taxing, zoning, debt issuance, and eminent domain (Burns 1994).

Burns (1994) views the consequences of institutional choice in terms of powers, services, financing, accountability, and participation. She argues that the first three relate to the structure of government while the latter two reflect the extent to which institutional choices reflect democratic values. This serves to illustrate how differing institutional arrangements result in varying organizational outcomes. In this regard, Burns (1994) implies special districts reflect routine-based, history-dependent organizations that exhibit path dependencies. 
General-purpose, special-purpose, non-profit, and for-profit institutional arrangements each exist as unique bundles of institutions. Each institutional arrangement is particular to the role of that institution. They also each possess a differing organizational form predicated on functional requirements (Mintzberg 1983). They exist as separate and distinct forms of organizational governance. The scope of interests and the arbitration of values largely drive the institutional form that emerges (Morgan et al. 2013). This reflects institutional choice that results from temporal proclivities.

General-purpose, special-purpose, non-profit, and for-profit organizations exist as creatures of state government by specifying the conditions under which these organizations can be created and operate. While they are the progeny of the state, the federal government also places restraints and constraints on the actions of organizations. This is the case since federal and state laws are the institutions that delineate the governing powers available to mobilize private resources for the common good. In this regard, state and federal laws "define the way in which citizens employed these governments achieve to their own private ends" (Burns 1994, 113).

Public general-purpose governments include counties, municipalities, cities, and towns. Burns (1994) states that new cities emerge when a mutually beneficial partnership arises between large groups of citizens desiring services and entrepreneurs intent on making a profit. The challenge, as Burns (1994) points out, is that the number of counties, cities, and towns has been in sharp decline. Morgan et al. (2013) advise that this institutional form will emerge when a common scope of interests coincides with the need for the political arbitration of values. 
Local general-purpose governments have traditionally been the primary provider of inclusive public goods and services like roads, police protection, and fire services. Local governments also provide an institutional mechanism for dealing with externalities like environmental pollution and common-pool resource management (Morgan et al. 2013). It is unlikely the polis would provide these goods and services absent the coercive power of local government (Olson 1971). General-purpose government receives funding largely through taxes and debt issues backed by their taxing powers. They can also receive grants from the federal government.

Morgan et al. (2013) concludes that one of the defining characteristics of generalpurpose governments is a rule-driven and process-oriented approach to providing good and services. General-purpose governance reflects the classical public administration philosophy in which the theories of political science, public policy, and organizational theory dominant (Morgan et al. 2013). The emphasis on hierarchical and professional control reflects the values of efficiency and effectiveness in achieving organizational outputs (Perrow 1986).

This results in a bureaucratic general-purpose organizational governance structure in which authority is hierarchal and centralized (Weber 1964). The focus is on providing citizens with equity in the provision of goods and services. General-purpose governments, moreover, that are embedded in democratic regimes are nominally accountable and responsive to the expressed wishes of their citizens (Manin, Przeworski, and Stokes 1999). The decline in the number of general-purpose governments suggests that the leadership approach and organizational values associated with classical public administration are no longer salient in meeting public needs. 
Private sector organizations include for-profit firms and corporations. Morgan et al. (2013) assert that a private sector institutional form emerges when parochial interests coincide with the market arbitration of values. Private sector organizations have historically enjoyed success in meeting public demand for exclusive goods and services when imbedded in legal institutions that place primacy on protecting private and perpetuating the rule of law (Fukuyama 2014). This is in large measure because the American political economy provides a relatively efficient mechanism for matching consumers with goods and services.

This is the case since the quantity of market goods is fixed, divisible, and, therefore, subject to private ownership and exclusive transfer rights (Olson 1971). Private sector organizations receive funding principally through proceeds from the sale of goods and services in a nominally open market (Olson 1971). Private sector firms provide goods and services to the private and public sector on a for-profit basis. Many public institutions purchase goods and services from private sector organizations using market mechanisms (Cooper 2003).

Morgan et al. (2013) propose that another one of the defining characteristics of private sector organizations is that they largely operate based on self-interest in meeting their preference for profits (Morgan et al. 2013). The focus is on flexibility and innovation in meeting customers' needs. This involves infusing the organization with an entrepreneurial spirit. This spirit empowers employees to satisfy customer demands in a flexible manner (Morgan et al. 2013). These firms, therefore, are results-oriented and mission-driven. In recent years, private firms have increasingly offered services to public sector organizations on a for-profit basis (Cooper 2003). 
The public sector has contracted with the private sector for a wide range of traditional services including the running of jails and the provision of sanitary services. This reflects the New Public Management (NPM) public administration philosophy in which the theories of rational choice and scientific management dominant (Morgan et al. 2013). The emphasis in NPM is on organizational performance and the use of the market and contracts to arbitrate values (Cooper 2003). Organizational performance in satisfying customers as efficiently and effectively as possible is the primary focus in this paradigm (Morgan et al. 2013).

Non-profit organizations represent "entities dedicated to mobilizing private initiative for the common good" (Salamon 2003, 2). The relatively recent growth in this sector is the result of a public reluctance to expand the size and scope of general or special-purpose government (Salamon 2003). Morgan et al. (2013) write that this institutional form emerges when a common scope of interests coincides with the market arbitration of values. Since the 1960s, non-profit organizations have provided an everincreasing range of public goods to citizens in the fields of healthcare, education, the arts, and social assistance.

Salamon (2003) concludes this increase is because while American citizens continue to demand public goods, they are unwilling to have governments provide the goods directly. As a result, non-profits have become a popular mechanism for delivering public goods and services. The result has been an explosion in the scale, roles, and functions of the non-profit sector. These organizations receive funding through federal, state, and local grants and contracts (Cooper 2003). They also receive philanthropic 
funding from private individuals or organizations (Salamon 2003). This sets them apart from the other institutional forms.

Morgan et al. (2013) assert that one of the defining characteristics of non-profit organizations is their voluntary commitment to meeting client needs through informal coordination. They provide flexibility for their target population. Non-profits seek to meet their clients' needs with minimal rules or questions asked (Salamon 2003). These value-driven organizations see themselves as fulfilling a moral duty to provide unmet social needs (Morgan et al. 2013). In this respect, normative values prevail when establishing organizational priorities.

The emergence and growth of the non-profit sector reflects the New Public Governance (NPG) public administration philosophy in which the theories of agency and networks dominant (Morgan et al. 2013). The emphasis is on the co-production of value by networks and partners through mutual agreement on actions (Mogren 2013). Value arbitration occurs through networks and relational contacts. These types of organizations have a "minicentric" orientation and an external focus on the local community (Morgan et al. 2013, 123).

Special districts are "independent local governments that generally perform only a few local government functions" (Burns 1994, 8). Excluded from this category are school districts. This is the case because the ubiquity of school districts skews the data such that all special districts would resemble school districts. Granular specificity on special districts as a distinct entity requires the segregating of data on school districts. Morgan et al. (2013) propose that the special district institutional form emerges when parochial interests coincide with the political arbitration of values. 
A special district "allows citizens to purchase additional levels of service that the government is not able to provide" (Morgan et al. 2013). They provide a wide range of public goods and services including housing, hospitals, public utilities, industrial development, public ports, cemeteries, and firefighting. It is worth noting that generalpurpose governments may also provide the same types of services. Special-purpose governments receive their funding largely through the services they provide, in addition to taxes and debt issuance.

One of the benefits of this institutional arrangement is that it provides consumers of special district goods and services some control over the price they pay (Morgan et al. 2013). This, however, raises questions of equity and makes it difficult to create a sense of community. Morgan et al. (2013) advise that one of the defining characteristics of special-purpose governments is their technical expertise and functional competence. Another is their narrow legal authority. Special-purpose governance reflects the New Public Management (NPM) philosophy that takes the business or market model as the standard for measuring government success (Morgan et al. 2013). The emphasis is on the delivery of efficient and effective services.

The ninety-eight public ports in Washington and Oregon provide the study population for observing organizational innovation in the public sector. These special districts use public funds to develop and/or operate publicly owned facilities that provide both recreational and commercial benefits to private citizens. Each port is, however, distinctive in the uses to which it puts public and private funding. Those located along the upper Columba River and Puget Sound support the international agriculture and natural 
resources trades while coastal ports support tourism, recreation, commercial, and sport fishing industries.

The deep-water facilities in Tacoma, Seattle, Portland, Astoria, and Coos Bay provide access vital to the development and expansion of agricultural and commodity markets in the US and Canada. The markets these ports serve are regional, national, and international in scope. While the establishment of each port was for a similar social purpose, they underwrite a wide assortment of economic sectors key to sustaining the Pacific Northwest economy and the quality of life in the region. In this regard, they serve as economic engines that provide regional access to global markets.

Oregon and Washington ports vary in size, organization, funding, mission, strengths, and challenges. There are 23 ports in Oregon State and 75 in Washington State. They each support state, regional, national, and international needs. They have the potential to serve as hubs of both commerce and innovation. This dissertation uses the Washington and Oregon public port authorities as the population for validating the proposed model. The unit of analysis for this study is the individual engaged in organizationally appropriate relationships that result in the co-production of innovation in the public sector.

This dissertation is an exercise in theory building. The analytical focus is on understanding social interactions and predicting when and under what conditions the coproduction of public sector innovation will occur. This dissertation, therefore, proposes and then puts at risk a theoretical model. Empirical observations serve to test the model's validity. This involves case studies using instances of innovation in the public port authorities of Washington and Oregon states. The goal of this dissertation is to increase 
understanding of co-production of innovation in the public sector. I hope to accomplish this by providing a useful explanatory framework for conceptualizing the phenomenon.

In advancing a coherent intellectual framework, this dissertation will contribute to the existing body of knowledge on public sector innovation. The objective of this research is to provide both theorists and practitioners alike the insights necessary to, for example, identify a set of practical tools for developing and sustaining the co-production of innovation within the public sector. The study of organizational innovation within the unique historical, political, and organizational context of public port authorities in Washington and Oregon adds to the body of knowledge on innovation. It provides additional insight into the theory and practice of the co-production of innovation in the public sector since no generally agreed-upon model of innovation in the public sector currently exists.

\section{Research Steps}

A theory is good to the extent it accurately mirrors the empirical domain it is attempting to describe (Dubin 1969). Judging a theory good, therefore, requires empirical research to validate the adequacy of the theoretical model. Freeman (1977) asserts, "As there is no one path to discovery, there is no one set of steps in research" (223). This dissertation, nevertheless, follows a path laid out in the research design literature for theory building (Dubin 1969; Freeman 1977; Yin 1994; Johnson and Joslyn 1995; Kumar 2011). What follows is a brief summary of the key components, or steps, of the empirically based research process that guided this dissertation. Figure 2 depicts this dissertations research steps. 
Although the steps outlined in Figure 2 appear to reflect a linear or sequential process, this is not the case. At its core, the process of discovery is iterative and nonsequential in nature. An epiphany awaits the researcher around every corner. New learning loops back to inform and reform previous understanding. Each step informs not only the proceeding steps, but the preceding steps as well. In other words, these steps loop back into previous steps. The result is a nuanced and flexible pathway to understanding the causal mechanisms of an exceedingly complex social phenomenon that moves in the general direction that figure 2 suggests.

Figure 2. Dissertation Research Steps

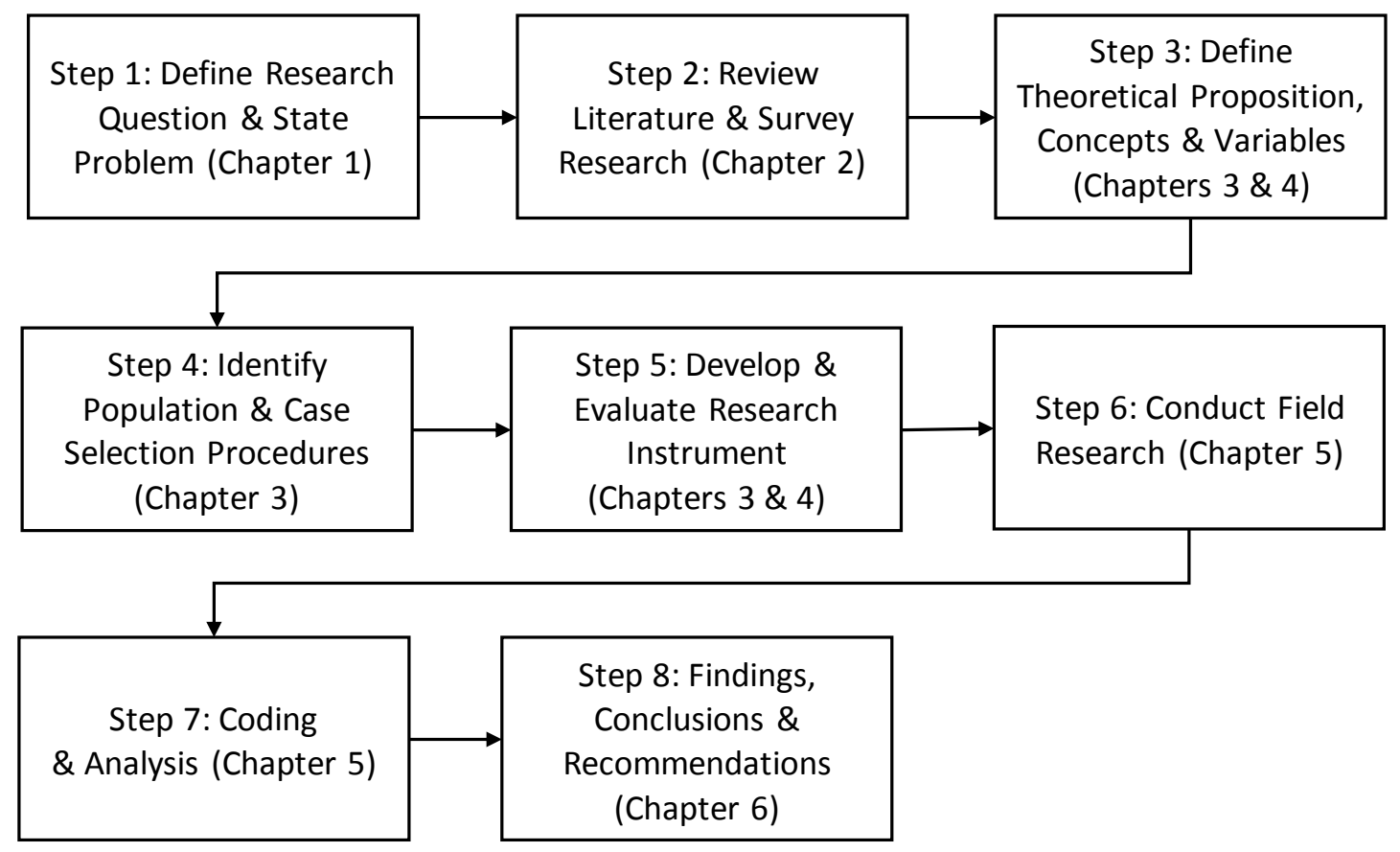

(Source: Freeman 1977; Yin 1994; Johnson and Joslyn 1995; Kumar 2011) 
The first step outlined the central research question. This step also involved identifying the problem that this dissertation solved. This is the focus of chapter 1. Step 2, the subject of chapter 2, outlined the salient results of a review of the literature. In other words, chapter 2 reported the results of a survey of previous research on this topic. Included in the survey was research literature on topics integral to answering the central research questions. This research served as the basis for developing this dissertations theoretical and conceptual framework. Since theory exists as a synthesis of a long succession of empirical results, this approach allows for the integration of earlier research findings (Dubin 1969).

The third step is to propose a theory. The proposed theory that this dissertation puts at risk describes and explains the phenomenon of co-produced public sector innovation. This theory benefits from the research reviewed in the previous step. Step 3 also defined the concepts, variables, and the units of measure used to operationalize the theory. Chapters 3 and 4 contain the results of step three. Step 4 identifies the population under study and establishes the sampling procedures. The outcome of this step in contained in chapter 3.

The fifth step was to draft, test, and validate the research instrument. Chapters 3 and 4 outline this process in detail. This step also included designing the coding manual for interpreting the interviews. Appendix B contains the case content codebook used in this dissertation. The codebook contains the numerical values assigned to different categories of responses to the interview questions. The sixth step is to conduct the field research. Chapter 5 discusses in detail the approach and activities involved in this step. 
The seventh step involved coding the interviews and analyzing the results. Chapter 5 presents the results of the coding and analysis in the case study format.

The eighth and final step is to present the research findings. This is the subject of Chapter 6 . This chapter explores the extent to which the model accurately portrays the observable world. In this regard, empirical data collected in the earlier steps serves as the basis for judgements related to adjusting, changing, or discarding the model. The concluding chapter also outlines the broader implications of the theory to the field of public administration and political science. This chapter postulates the generalizability of the theoretic framework and the research conclusions. Chapter 6 also presents suggestions for the direction of future research in this field.

\section{Assumptions and Limitations}

Theory and practice requires a flexible, feasible, and adoptable framework for public sector innovation (Van de Ven, Harold and Poole 1989a; Walker 2008; Bason 2010). What the literature on public sector innovation reveals, however, is that an agreedupon and empirically tested theory regarding the co-production of innovation in the public sector is missing. This dissertation seeks to fill this void. It does so by providing an empirically valid model that provides fresh insight into the conditions and causes of the co-production of public sector innovation.

The goal of this study is to bridge the gap between theory and practice. This dissertation accomplishes this by defining the conditions that lead to the co-production of public sector innovation. These conditions are termed the antecedents. This study defines and measures ten independent variables. These independent variables serve to indicate 
the presence of the antecedents that open the pathway to the co-production of public sector innovation. The independent variables are present when the dependent variable of public sector innovation emerges. This research agenda is, therefore, directly relevant to the practice of public sector innovation. It employs a deductive approach that uses a grounded theory to describe the conditions present when innovation occurs. Empirical observation validates the model.

The empirical setting for this study is special-purpose governments or "special districts." These two terms are interchangeable for the purpose of this study and represent the same type or form of governing institution. These types of governmental organizations typically focus on delivering one type or a discreetly bounded portfolio of public goods or services (Burns 1994). Special districts are distinct from general-purpose governments like counties and municipalities such that the latter two provide a wider range of public goods and services (Foster 1997).

For the proposed model to represent the phenomenon it is describing, it must have boundaries that encompass innovation in the public sector (Dubin 1969). This dissertation established such boundaries. It did so by limiting the population of special districts to one particular type: public marine ports. This had the effect of limiting the scope or extent of the study. Limiting the study population to one particular type of special district also had the potential of limiting the generalizability of the findings to other types of special districts.

Causal conditions could have emerged that are not translatable to the coproduction of innovation in special districts other than public marine ports. It is also conceivable that this study could have failed to reveal antecedents that are consistently 
present when this phenomenon is manifest in other types of government organizations including general-purpose governments. Chapter 2 takes up the subject of generalization. Generalizing in the context of this dissertation is the process of extending theoretic framework, findings, conclusions, and recommendations to other organizational types or forms (Kuman 2011).

Selecting a study population and gaining access to that population is often a unique challenge (Kumar 2011). This study makes use of a personal and professional network of individuals to gain access to the study population. I have direct involvement in this study population through my affiliation with public maritime ports in Oregon and Washington State. As such, I am a participant in this area of research. There is, therefore, a potential for bias and a subsequent loss of subjectivity in this study (Kumar 2011). The research design provided protection against these threats to the validity and reliability of the dissertation.

This study has a number of safeguards to ensure that personal affiliations do not compromise the results of this study. These safeguards include, for example, the development of a standard interview guide in which each participant receives identical questions and probes. Appendix A contains the list of questions used to conduct this research. Another possible protection to insure an objective, value-free, and unbiased approach is intersubjectivity. In this regard, the achievement of intersubjectivity occurred through shared cognition and consensus around ideas about public sector innovation and the relationships that the model suggested.

The study population selected for this dissertation is the twenty-three public marine ports in Oregon State and the seventy-five ports in Washington State. This study 
is limited to the geographic region of the Pacific Northwest (PNW)—although important exceptions to the regional affiliation of the ports exist. This study excludes, for example, foreign ports in Canada and ports in the neighboring state of California. The primary purpose of an individual port can vary from local commercial or sport fishing to international commerce in bulk or general commodities.

Public marine ports are special districts established to foster economic development. One mechanism for achieving this common good is though international trade and commerce. Public ports also support local tourism, recreation, and commercial or sport fishing. With regard to business lines it is, therefore, impossible to assume that a "generic" or "standard" public maritime port exists in Oregon or Washington. Although the public maritime ports in this study provide a uniquely appropriate setting, future research is required to rigorously test this model and determine the generalizability of the finding.

The small pool of Oregon and Washington ports represents a potential study limitation. This could have important implications for any conclusions drawn regarding the theoretical framework, specifically regarding limitations on the generalizability of the findings, conclusions, and recommendations. The size and primary function of the various public marine ports in the study population also has the potential to create further limitations. In this context, the size of a port relates to the type and amount of resources available. This can include real estate, financing, social, or political capital.

There is a danger, furthermore, in generalizing findings, conclusions, and recommendations from such a highly contextualized approach. This primarily relates to the validity and reliability of the study's findings. This study used a non-random design 
to select cases. This is a targeted approach to studying this phenomenon. This approach is also in alignment with this dissertations theory development protocol. In this dissertation, the cases selected were from a population in which the phenomenon under study manifest.

One of the factors considered in selecting the sample was the size of port. There was a division of the ports into three broad categories: small, medium, and large. The annual revenue of the port authority determines the size of a port for the purposes of this study because annual revenue is a strong indicator of the size of a port. This is the case since it reflects both the volume and value of the ports activities. Higher volumes and values result in higher annual revenue and reflect a larger potential resource base. Annual revenue also provides some indication of the economic impact of the port.

This research project involved interviews and other data collection methodologies tied to human subjects and, therefore, fell under the purview of the Portland State University Institutional Review Board (IRB). The PSU IRB granted the necessary approvals and provided oversight for this study. In addition, dissertation committee members oversaw the research to ensure that the appropriate protections, protocols, and timelines were in place. Each study participant granted his or her informed consent prior to participation in this study. In this manner, the study protocols provided maximum protection to the study participants. These protections served to guarantee the generation of valid and reliable study results.

There were a number of challenges faced during this study. There was a risk that the study participants may have given socially or politically correct answers regardless of the reality on the ground. To the extent possible, the study protocol served to guard 
against this by ensuring the confidentiality of each participant. The research protocol ensured the privacy and secrecy of the identities and responses of the subjects. There was also the danger that the study participants may reveal potentially sensitive information. In this case, the confidentiality through anonymity granted each study participant served to minimize, to the extent possible, any resulting harm.

This dissertation, while limited in focus, developed greater insight into the internal and external antecedents of organizational innovation. The value of this research is in providing a model for the co-production of public sector innovation that aligns closely with what the practitioner experiences in the real world. I plan to use the results of this dissertation to present, publish, disseminate, or otherwise share insights on the coproduction of public sector innovation. This is an important contribution to the fields of political science, public policy, and public administration. This is the case since there is an increasing appetite for insight into the mechanism of public sector innovation (Sorensen and Torfing 2012).

Technology can play a dual role as both a driver of innovation and driven by innovation. Technology is ubiquitous and, therefore, it imbeds deeply in both the process of innovation and its results. Weerawarden (2003) concludes that innovation research grants technological innovation primacy of place. The process of technological innovation, however, is a field unto itself. This in no way denigrates or rejects the important role that technological innovation plays. It has both necessary and beneficial social effects. It is, however, beyond the scope of this dissertation. This study, therefore, excludes the subject of technological innovation. 


\section{The Road Ahead: A Summary of the Dissertation}

This dissertation seeks to understand the co-production of innovation at the organizational level. Organizations represent the near-decomposability means of securing the benefits of coordinating collective action while holding down the costs by an appropriate division of labor among sub-units (Simon 2000). They also provide a means of managing change, chaos, and transformation (Morgan 2006). Organizational innovation, furthermore, offers an opportunity for adaption to change critical for organizational survival. Organizations also provide the framework for thinking and acting that is necessary if individuals are to provide social value (Denhardt et al. 2013). Organizations, therefore, provide the context for what follows.

Chapter 1 of this dissertation briefly describes and summarizes the research agenda for this dissertation. It frames the study and brings into focus the approach to the exploration of the co-production of innovation in the public sector contained in this dissertation. The chapter opens by providing some background on the study of innovation in the public sector. In doing so, it presents a perspective on the phenomenon that argues that innovation is necessary to foster good governance. Chapter 1 also includes a presentation of the framework for understanding and predicting this phenomenon. There is also an exploration of the nature of public versus private sector innovation presented in this chapter.

The problem facing the public sector is that no generally agreed-upon or empirically tested theory of public sector innovation exists. This dissertation fills this gap by providing an explanatory model that will give theorists and practitioners alike unique insights into this phenomenon. In other words, this model explains the causality of public 
sector innovation. Chapter 1 presents the model's implied theoretical basis. This dissertation employed the case study approach that empirically validates a model that explained what causes innovation to occur in the public space. Comparative case studies represent the best methodologic approach to building such a theory (Yin 1994). This is the case because the co-production of public sector innovation is highly complex and contextual in nature.

Chapter 1 contains an outline of the role and importance of special districts in public governance and then presents the empirical setting and study population for this dissertation. The special district public maritime ports in the states of Oregon and Washington provide a representative venue for this study of innovation. This chapter also presents the research steps and addresses several assumptions and limitations inherent in this study. Chapter 1 concludes with an overview of the proceeding chapters of this dissertation.

Chapter 2 contains the results of a review of the relevant literature, the objective of which is to bring the subject of public sector innovation into focus. This survey of the literature serves to frame salient issues around the co-production of innovation in the public sector. This chapter provides a point of departure for this study. Chapter 2 presents the results of an exploration of innovation at, below, and above the organizational level. It also contains an examination of several additional issues including the measurement of innovation and the relationship between leadership, organizational culture, and innovation. This chapter also contains a summary of the literature on co-production and special districts. 
Three general themes animate the research findings on innovation. The first is that institutions and organizations matter. The second is that leadership is critical to the process of innovation. The third is the need for the active and often intimate involvement of customers or clients in the innovation process. While existing theories of innovation touch on each of these themes to varying degrees, none provides a clear depiction of the dynamics of co-producing innovation in the public sector. These theories, moreover, remain devoid of empirical evidence.

Chapter 3 opens with a section on the definition of relevant terms used in this dissertation. It then presents a conceptualization of the integrated model of organizational innovation this dissertation subsequently puts at risk through empirical observation. The model suggests that the co-production of organizational innovation is dependent on the actions of organizational leaders, the organization itself, and the customer or client. This model, furthermore, embeds in a strategic environment conditioned by the prevailing political economy.

A research question is an answerable inquiry into a specific topic, issue, or concern (Kumar 2011). It represented the initial step in this dissertation. The research question this dissertation seeks to answer is how leaders at all levels of the organization, the organization within which they exercise leadership, and their customers or clients interact to co-produce innovation in the public sector. Put another way, what are the causal mechanisms of innovation? The presence of certain antecedents related to leadership, the organization, and their customers or clients provides an explanation. This model is, therefore, useful in conceptualizing this phenomenon. 
The causal relationships the model suggests occur within a strategic environment that mediates the actions of individuals and organizations. These same individuals and organizations can also shape the strategic environment. The model posits the existence of antecedents that are present when public sector innovation occurs. Variables exist that serve as indicators of antecedents. These independent variables, furthermore, are subject to empirical observation. It is possible, therefore, to operationalize the model through these indicator variables. In other words, the identification and measurement of certain variables serve as indicators of the antecedents. These antecedents serve as the pathway to the co-production of innovation in the public sector.

Chapter 3 presents an empirically oriented approach to validating the model. This chapter introduces a composite index for measuring the degree of innovation that a researcher should expect to observe in an organization. This is the public sector innovation organizational index (PSIOI). The composite index uses four attributes of the variable to measure the concept of innovation. These attributes include resilience, quality, accessibility, and efficiency. The proposed index seeks to answer the question of how much innovation the researcher should expect to observe in a public sector organization.

The organizational antecedents of public sector innovation (OAPSI) rating introduces a methodological approach to empirically measuring actual public sector innovation. The protocols for creating the rating requires the researcher to identify and assign values to variables that serve to operationalize the antecedents. The measurement of the variables serve to indicate that the model is operative. Both the PSIOI and the OAPSI rating use an ordinal scale. This chapter concludes with a brief discussion of ethical considerations related to this study and a statement of disclosure. 
Chapter 4 presents a description of the process used in this dissertation to operationalize the model. This chapter contains an outline of the strategy, approach, and design employed in this dissertation. This chapter also includes an explanation of the tools, techniques, and procedures used in the study. The intent of this theory building exercise is to provide a holistic perspective of the phenomenon under study. This chapter, therefore, contains a summary of the methodological approach to instrumentation and data collection. It provides sufficient detail to give future researchers a roadmap for replicating this study. Chapter 4 insures transparence in the way the theoretical model is constructed and validated.

This dissertation uses naturalistic inquiry and emergent design flexibility to understand the phenomenon (Patton 2002). Data collection occurred using primary and secondary sources. The principle primary source of data was semi-structured, open-ended interviews with additional probes to yield insight into the perceptions, knowledge, and opinions of individuals involved in innovation in the public sector. This study also relied on field observations and documentarian data collected coincidentally with the interview process. This data served to provide additional analytical insights.

In chapter 4, particular attention is devoted to explaining the methodological approach used to formulate the public sector innovation organizational index (PSIOI) and the organizational antecedents of public sector innovation (OAPSI) rating. The former provides an aggregate summary of the expected innovation and the latter a similar measure of actual organizational innovation. The aggregation or summary of the data serves to offer unique insight into this phenomenon. Chapter 4 concludes with a description of the layout and organization of the cases presented in chapter 5 . 
Chapter 5 presents a series of three case studies involving public sector innovation. This dissertation used a strategy that employed inductive analysis and creative synthesis to express the results of the data collection and analysis in a narrative case study format. George and Bennett (2005) argue that, "There is a growing consensus that the strongest means of drawing inferences from case studies is the use of a combination of within-case analysis and cross-case comparisons" (18). This dissertation follows their recommendation. This chapter presents a within-case analysis of each case in isolation, followed by a cross-case comparison involving the three cases studies.

Chapter 6 summarizes and concludes the dissertation. The chapter accomplishes this by presenting the synthesized results of the comparative case study analysis. Chapter 6 opens with an evaluation of the methodological approach taken to data collection and analysis. The findings, conclusions, and recommendations that emerged from the case studies are the focal point of this chapter. The findings led to a revision of the model. The chapter, therefore, contains the revised theoretic framework. Chapter 6 also includes a discussion of the implications and the value of this research to practitioners and theorists of public sector innovation.

Chapter 6 contains suggested actions for enhancing our understanding of public sector innovation and lays out a research agenda for the future. This study raises, but does not fully answer, several questions related to the generalizability of the findings. It is up to future research to test the model proposed in this dissertation in a rigorous manner. 


\section{CHAPTER 2: SETTING THE STAGE}

While organizational innovation in the private sector is the subject of intense academic interest the study of this phenomenon in the public sector pales in comparison (Shin and McClomb 1998; Walker 2008). Innovation is vital to employing resources in a time when the complexity of delivering public goods and services strains organizational capacities (Micheli 2012). The challenges that face public sector organizations today are often transnational in nature and span multiple jurisdictions. Today's leaders confront a complex mix of problems that crosscut traditional environmental, economic, social, and political issues. Today's challenges demand that public sector organizations nurture innovation on a broad scale to remain relevant.

Past research reflects a bias towards technological innovation (Weerawardena 2003). Meyer and Goes (1988) warn that, "Innovations embodied in new equipment have few inherent attributes that can be ascertained unequivocally without reference to a specific organization at a specific time" (900). This leads them to conclude that technological innovation often has unintended consequences that result from second and third order effects. Technology may solve problems or create new opportunities. It may also place well-established institutional norms and regimes in jeopardy (Meyer 1985). While technology can play a role enabling public sector innovation, it is not the focus of this dissertation.

An organization's leadership plays a central role in fostering innovation and change (Vasccaro et al. 2012; Meyer and Goes 1988). Likewise, the organization can either resist or be an impetus for innovation and change. Clients or customers of the 
organization provide the organization and their leaders opportunities to collaborate in the co-creation of public sector innovation. This chapter seeks to frame what is known about the theoretical and practical relationship between public sector innovation, leadership, the organization, and its customers or clients in the co-production of innovation. The goal is to provide practitioners of public sector governance insight into the contemporary literature on the theory and practice of innovation. This chapter provides the foundation and serves as the point of departure for this dissertation.

The focus of this study is on the co-production of innovation within the organizational context. The first section of this chapter introduces this topic. The second section reviews and analyzes the literature on organizational-level innovation within the public sector. While this section is anchored in the unique contributions of Zaltman, Duncan, and Holbek (1973), Van de Ven (1989a), and Bason (2010) it also highlight the contribution of a number of other theorists. The concepts set forth by these authors provide a starting point for the exploration of this topic. The third section of this chapter proves a synthesis and summary of some of the key conclusions drawn from the literature.

The fourth section of this chapter explores efforts to quantify or measure public sector innovation. It also touches upon why this has recently taken on a new level of urgency. The next section considers how innovation manifests below the organizational level. In this section, the work-group and individual levels of innovation are considered. Innovation at levels above the organization is topic of the sixth section. This section specifically considers the emerging "systems of innovation" theoretical framework. The seventh section considers the relationship between leadership and innovation. Public 
sector co-production is the topic of section eight. The next two sections review the literature on special districts. These unique governmental institutions represent the empirical setting for this study. A brief conclusion follows.

\section{Introduction}

Porter (1990) posits that organizations create a competitive advantage over other organizations through the act of innovation. In this context, "innovation... is defined broadly, to include both improvements in technology and better methods or ways of doing things" (Porter 1990, 45). This approach assigns innovation the central role of economic engine in a market-based economy. Porter's theory of competitive advantage, moreover, focuses exclusively on the manufacturing sector to the exclusion of services. It is the latter, however, that is prevalent in the public sector (Nankervis 2005). There is criticism that this framework is, therefore, inappropriate for the public sector. Osborne and Brown (2013) advise that this conceptualization has led to "a flawed understanding of the nature of innovation" in public services (2).

Walker (2008) defines public sector organizational innovation "as a process through which new ideas, objects, and practices are created, developed or reinvented, and which are new for the unit of adoption" (592). The results of research into innovation suggest that the strategy and structure of an organization, more so than its size, plays a role in fostering the range or degree of innovation (Ettlie, Bridges and O'Keefe 1984). This viewpoint posits that it is possible to reflect innovation on a categorical or classification scale. This is important because it infers that differing approaches or innovation strategies can exist. Contemporary research holds that a typology of 
innovation is present in the public sector as well (Damanpour 1987; Walker 2008). This means in practice is that researches tend to classify innovation into differing general types.

Emerging from the recent literature is the proposition that the degree and type of public sector innovation is salient in determining the intensity of innovation (Weerawardena 2003). Weerawardena (2003) and Walker (2008) suggest that the magnitude of a temporal or spatial quantity of innovation is capable of being known and thereby quantified. This creates a conceptual framework for understanding innovation in which both the degree and type of innovation play a central role in describing this phenomenon. Together these two factors yield a measure of innovative intensity that researchers have yet to quantify (Weerawardena 2003). Regardless, this lends support to the contention that the intensity of innovation relates to an organizations ability to sustain its competitive advantage (Weerawardena 2003; Walker 2008).

Contemporary innovation theory at the organizational level differentiates between two types of innovation ranging from incremental to radical (Hage 1980; Ettlie, Bridges and O'Keefe 1984). The former is incremental in the sense that it involves discontinuous change. It is limited to the transformation of products or services resulting from incremental process adoption (Hage 1980). In this regard, the underlying process remains fundamentally unchanged. The research suggests radical innovation is also discontinuous in nature manifesting in altogether new processes that represent a risky departure from past practice (Ettlie, Bridges and O'Keefe 1984). Embedded in the concept of innovation is, therefore, risk and change. 
Osborne and Brown (2003) conclude there is a danger in conflating incremental improvement with incremental innovation. Incremental improvement represents continuous change in which the current products, services, or processes remain fundamentally intact (Hartley 2005). Incremental innovation, on the other hand, is discontinuous such that the products and services that came before are adapted to meet emerging social needs (Ettlie, Bridges and O'Keefe 1984). This is akin to the emergence of a fundamentally new insight into a phenomenon such that a perception of novelty emerges.

Ideas of change and continuity around processes and practices tie closely to the concept of innovation. Martin and Gartland (2003) suggest that path dependency provides a set of options at a given time that are the result of past decisions. This is the case even though the circumstances may no longer be relevant—in other words, history matters. Small events can also create disproportionate amplification at later points and under differing circumstances. Cohen (1972) suggests this latter phenomenon is the "strong" form of path dependency that, while narrower in scope than the historical force, often provides a more satisfying explanation of outcomes over time.

Porter (1990) asserts that the nature of most innovation is incremental rather than radical. It occurs over time through the application of newly acquired skills, knowledge, or insights. Walker (2008) contends this is characteristic of an evolutionary approach to innovation. This approach is most likely to occur under conditions of complexity, formalization, and decentralization (Ettlie, Bridges and O'Keefe 1984). Other research concludes that radical innovation emerges under informal and centralized organizational conditions. Radical innovation, for example, may result from a technological 
breakthrough or a radically new and disruptive combination of existing technologies (Walker 2008).

In addition to the evolutionary approach to innovation, Nagji and Tuff (2012) spotlight the benefit of organizations adopting a portfolio strategy to innovation. This involves a centralized management approach in which the organizations leaders attempt to identify, prioritize, authorize, manage, and control innovation in such a manner that it achieves specific strategic objectives. They advise simultaneously investing in initiatives that focus on serving current markets, expanding existing markets, and creating entirely new markets. Although they admit, the exact ratios of their portfolios vary in practice.

Nagji and Tuff (2012) advance the notion that "firms that outperform their peers tend to allocate their investments in a certain ratio: $70 \%$ safe bets in the core, $20 \%$ to less sure things in adjacent spaces, and 10\% to high-risk transformational initiatives" (5). While governments tend to have few peers in the spaces in which they operate the research of Nagji and Tuff (2012) suggest a rough quantification of the allocation of resources that an organization should devote to public sector innovation. Their writings also point to the need for governing institution to refresh their portfolio of innovation in order to solve complex and multifaceted social problems.

Types of innovation include those related to product, process, or organization (Weerawardena 2003). This illustrates the additional nuance that researches continue to add to the study of public sector innovation. Product innovations attach to goods that the public consumes, while the creation of such products and services is the focus of process innovation. Organizational innovation relates to the embedded social structure and management processes. They include, for example, the degree of centralization, 
formalization, or specialization that characterizes the organization (Walker 2008). Within the context of public sector innovation Walker (2008) expands the list to include service and marketization innovation.

Walker (2008) posits that service innovation is akin to product innovation. He goes on to advise that the marketization of innovation infuse government contracting, the purpose of which is income or revenue generation. Damanpour (1987) identifies ancillary innovation to differentiate cross-boundary innovation from other types. This relates to innovations over which a public sector organization has limited control. It includes, for example, the development of local strategies by nominally independent entities or the implementation of statutory partnerships (Hartley 2005). It can also include serving new clients or customers (Hartley 2005).

Walker (2008) posits that distinguishing between innovation types is a necessary, but not sufficient, requirement for understanding the process of innovation. This is the case since organizational and environmental characteristics appear to modulate the nature of the relationships between the innovation types (Walker 2008). This suggests that innovation is an inherently dynamic process and that understanding the dynamics requires a holistic understanding of the organization. This further reinforces the viewpoint that organizational culture plays a dominant role in creating an environment in which public sector innovation flourishes (Morgan 2006).

Hartley (2005) posits that in relation to innovation, "different conceptions of governance and public management... have implications for the role of policy-makers, managers and the population" (29). Recent theorists have relied on this observation to identify the underlying mental model (or paradigm) of governance as a type of innovation 
(Bessant, Hughes and Richards n.d.). It is interesting to note that it appears this approach is the basis of theorizing the existence of co-production. In this regard, co-production assumes the active, rather than passive, involvement by customers or clients in the delivery of public goods and services (Parks et al. 1981).

Contemporary research implies that there are several characteristics of innovation that define the manner of its perception (Hartley 2005; Damanpour and Schneider 2009; Fuglsang and Sørensen 2011). These characteristics exist separate and apart from the degree or type of innovation. In this regard, it is important not to conflate these three concepts: degree, type, and characteristic of innovation. Characteristics include, for example, the proposition that innovation can result from either intentionally planned or unplanned tinkering (Fuglsang and Sørensen 2011). It is also possible to characterize innovation as emerging in an a posteriori or ad hoc manner. Zaltman, Duncan, and Holbek (1973) identified, moreover, twenty-two attributes that characterize innovation.

One of the central themes to emerge from the literature is that the scale or scope of innovation can change (Moore and Wesley 2011). The manner in which innovation is considered, therefore, varies depending on the level. The hierarchal perspective largely dominated public administration thinking during the late twentieth century. This legacy continues to influence contemporary perspectives on organizations. The literature implies that innovation occurs on differing plains including the work-group, network, organizational, institutional, and societal levels (Drucker 1998). All this serves to layer on additional layers of complexity to the study of public sector innovation.

The variance in the scale or scope of innovation requires a close reading of the literature to ascertain the relative unit of analysis that the author is employing. A review 
of the literature reveals that different levels tend to accentuate different aspects of innovation. At the work-group level, for example, the focus is on micro-level resource distributions and individual or group routines (West et al. 2003; King and Anderson 1995; Wallerstein et al. 19). While at the social level, the salient aspects include culture, legal regimes, and macro-economic resource distributions (Freeman 1987). All this suggests that the higher the level of innovation under consideration the greater the need to focus on the strategic aspects of innovation (Nagji and Tuff 2012).

\section{Innovation at the Organizational Level}

This section consists of a review and analysis of the literature on innovation at the organizational level. While the central focus is upon the unique contribution of three canonical theoretical conceptualizations of public sector innovation, it also highlights others. The first conceptualization considered is that of Zaltman, Duncan, and Holbek (1973). Their important contribution was in fusing innovation with implementation. In doing so, their work is valuable to understanding of the complex nature of innovation. In addition, they provide important insights into the attributes of innovation.

The second is the conceptualization of Van de Ven, Angle, and Poole (1989). Their unique contribution was to provide an integrating framework that attempted to make sense of the often conflicting and contradictory nature of innovation. They accomplished this by positing a metatheory of innovation. Their pioneering work developing a theory of theories at the University of Minnesota expanded our understanding of the complex interdependencies leading to innovation. The third is the conceptualization of Bason (2010). He presents a contemporary approach to the 
phenomenon. Bason (2010) presents a prescriptive, rather than descriptive, approach to innovation in the public sector.

Other academics have also made significant contributions to our understanding of public sector innovation. The work of these three theorists, however, represent the best contemporary reflection of the state of the literature on public sector innovation. As such, they provide a superlatively representative cross-section of research on this subject. These theorists also parallel, to a large degree, the evolution in the positivism within the philosophy of the social sciences. In other words, they chronical the development of how complex social phenomenon is understood. The juxtaposition of these three conceptual approaches is also useful in drawing distinctions between subsequent theories. They serve as the historical touchstone for understanding this phenomenon.

Early theories of innovation depict it as a linear process within the organization involving rational decision-making (Weber 1964). From this viewpoint, structural variables affect the outcome of organizational activities. Organic and mechanistic structures, moreover, have an important impact on innovation (Burns and Stocker 1961). Structural organization theory provides the point of departure for understanding the organization and its role in shaping innovation (Shafritz, Ott, and Jang 2011). Zaltman, Duncan, and Holbek (1973) exemplify this rational-linear approach to understanding innovation.

Zaltman, Duncan, and Holbek (1973) define innovation as "any idea, practice, or material artifact perceived to be new by the relevant unit of adoption" (158). In this context, innovation differs from change. In this regard, Zaltman, Duncan, and Holbek (1973) define change as an alteration of the structure or functioning of an existing social 
system. Innovation, therefore, causes change. Only the perception of new change, however, results in innovation. In other words, change and innovation coexist to a certain degree. In this construct, they dynamically interact in the space they simultaneous occupy. Zaltman, Duncan, and Holbek (1973) characterize the phenomenon as progressing through a series of steps that culminate in innovation.

Zaltman, Duncan, and Holbek (1973) maintain that there exists a creationadaption duality. Their model of innovation, therefore, proceeds in two stages from initiation through implementation. These stages each have associated decision sub-stages. Therefore, for example, the initiation stage consists of knowledge-awareness, attitude formation, and decision sub-stages. The implementation stage controls the process of initiating and sustaining the innovation. This later stage consists of an initial implementation sub-stage followed by a sustainment sub-stage.

The focus of Zaltman, Duncan, and Holbek (1973) was on the innovation process at the level of the organization. They identified three structural variables-complexity, formalization, and centralization - that are operative in their two-stage, five sub-stage model of innovation. Complexity, which is a form of diversity, emanates from the greater number and variation of occupational specialties and professionals in organizations with differing task structures (Mintzberg 1983). Formalization is akin to bureaucracy (Weber 1961) and centralization relates to the level at which the organization vests authority and decision-making power.

Zaltman, Duncan, and Holbek (1973) argued that the magnitude and direction of each variable changed depending on the decision stage. For example, they posit that the best coupling in the initiation stage is high complexity with low formalization and 
centralization. This is because of the diversity of information and viewpoints inherent in complexity and the need for low bureaucratic barriers. The converse is true in the implementation stage. In this stage, formalization and centralization provide the requisite structure for innovation. Likewise, a lower level of complexity provides greater organizational alignment since there is little in the way of controversy.

Because of the variability in the magnitude and direction of each variable, Zaltman, Duncan, and Holbek (1973) posit mediating factors. These factors are operative in the space between the two primary stages. These include having, respectively, a high capacity for effective interpersonal relations that involves dealing positively with conflict. In their model, the individual is an independent rational actor. In this regard, the process of legitimizing conflict and directly confronting areas of disagreement leads to consensus. This model views organizational change and innovation as a linear-as opposed to iterative-process.

This does not mean that organizations will not resist change. Indeed, the model suggests resistance to change will occur at both the individual and organizational level. The nature of resistance, however, changes throughout the model. For example, during the initial implementation sub-stage resistance may emerge at the organizational level to alter the innovation. When this happens, mediating factors are critical to overcoming resistance regardless of its nature. This model serves to highlight and further reinforce the complex nature of the phenomenon.

The theoretical model that Zaltman, Duncan, and Holbek (1973) propose has the benefit of focusing on an analysis of processes and, in so doing, contributes significantly to understanding this phenomenon. A number of attributes of innovation inhabit the 
model. These attributes relate independently to each of the five decision sub-stages. The larger setting in which innovation occurs provides context to the model and allows it to acquire a more complete meaning. The attributes operational in the knowledge sub-stage include the communicability of the innovation, its point of origin, and the presence of gatekeepers.

The Zaltman, Duncan, and Holbek (1973) model is highly contextual and situationally dependent. A large number of gatekeepers, for example, may either inhibit the likelihood of success or provide alternative channels for innovation to emerge. At the attitude formation sub-stage, financial cost, investment return, efficiency, risk, uncertainty, compatibility, complexity, and status quo ante are likely areas of consideration. Therefore, for example, the extent to which the proposed innovation is compatible with organizational norms affects the level of resistance one can expect to encounter.

The decision sub-stage involves cost, scientific status, perceived relative advantage, and commitment. Scientific status relates to the extent an innovation is reliable, valid, generalizable, and internally consistent. In the initial implementation substage the impact of interpersonal relationships, along with the ability to terminate the activity, and the private versus public nature of the innovation are operative. The latter attribute, for example, relates to the extent the good or service is exclusive or inclusive (Olson 1971). The susceptibility of an innovation to successive modifications and the respective capacity to innovate at the gateway are operative in the final phase. Gateway capacity is the extent to which the adoption of the innovation opens up avenues for the adoption of subsequent innovation. 
This mechanistic view of organizational innovation faced challenges from those viewing innovation as a complex iterative process in which cultural variables affect organizational outcomes. Indeed, Van de Ven and Poole (1989) advise that no single theory is sufficient to account for the complexity and diversity of the processes and patterns of innovation. In response, they proposed a metatheory specifying the conditions under which various theories or models best explain the phenomenon of innovation. This framework compares models of innovation according to the level of analysis (either global or local) and type of theory (historical, functional, or emergent process motors) to posit the contingencies under which each model holds.

The development of innovation, according to Van de Ven and Poole (1989), occurs on the local and global levels. Their approach seeks to provide a universal slant to understanding innovation. On the local level, models operate within the context of immediate action and exhibit a short-term focus. Global models, conversely, relate to the long range and overall holistic view of the process of innovation. While differing situations or contingencies determine which model to apply. Van de Ven and Poole (1989), nevertheless, propose three rules (type, temporal, and spatial) for determining when to switch between models. In other words, the three rules define the contingencies that apply to determining which model applies.

The first type of theory which Van de Ven and Poole (1989) advance is one in which a "historical motor of development" is operative (644). From this perspective, they posit that the process of innovation differs to the degree actions are constrained or restrained by institutional rules or natural programs. These exist because of logical necessity, institutional requirements, and biologic or natural law typologies. The latter 
views social change as predicated on continual evolution. Innovation, in other words, is a contextual social phenomenon subject to path dependencies and punctuated equilibrium.

Logical necessity relates to the natural order in which individuals or organizations pursue the process of innovation while institutional requirements reflect the historical modalities in which organizations operationalize innovation. This is consistent, for example, with Roethlisberger's (1941) observation that the endogenous development and stimulus-response modalities become operative under conditions of logical necessity at the local level. In this context, theories provide an opportunity to develop an understanding of the social relationships that underlie the phenomenon under study.

Van de Ven and Poole (1989) reason that the historical motor is applicable to innovation occurring in highly developed institutions. In this context, "the nature of the historical motor varies according to the nature of the relevant institution" (653). Using their example the sequence of steps required to produce legislative action reflects the order of stages in the global model while the local model reflects political negotiations. In this context, therefore, it is possible to view incrementalism as but one possible model for explaining the emergence of innovation in the political sphere (Lindblom 1959).

The "functional motor of development" is the second type of theory Van de Ven and Poole (1989) classify. In it, innovation processes differ to the degree to which there is a rational direction of action towards a functional outcome. Basic to this motor is the accumulation of power and resources necessary for innovation and, therefore, it proceeds from accumulation relationships, dependency relations, peer relations, and dominance typologies. The latter three relate to the level of power and resource asymmetry, which exists between the organization attempting innovation and its potential partners. 
As the name implies, accumulation relationships result from the attempt to gather power and resources from the external environment. Morgan (2006) argues that power is the central medium through which the resolution of conflicting interests occurs. Therefore, for example, a governmental sub-unit exists in a dominant relation to their contractors. Van de Ven and Poole (1989) posit a functional motor applies when key participants in the innovation process agree about the goals and end state of innovation. This can occur through institutional isomorphism (DiMaggio and Powell 1983).

The final development motor is the "emergent process." In this process, the progression of innovation differs to the extent actions arise almost randomly through either a socially constructed or a seemingly random set of occurrences. Variation exist in terms of evolutionary, dialectical, or enactment modalities. Evolution reflects a probabilistic process of variation and selection. Dialectical models result from the synthesis of countervailing theses. An example of this on the societal level is the emergence of structuration theory. This concept emerged from efforts to reconcile functionalist and behaviorist theories.

Van de Ven and Poole (1989) hold that enactment models, conversely, result from organizations enacting scripts. Perhaps the best know example is the garbage can model of choice (Cohen, March and Olsen 1972). This paradigm is only operative on a local level. The emergent process model holds when the conditions for the historical and functional motors are inoperative. Van de Ven and Poole (1989) posit an emergent process motor holds in the absence of strong institutional context. This is also the case when agreement regarding the goals or end state of innovation is missing. 
There are three points worth considering when reviewing the research up to this point. First, the research is primarily US-centric. While the organizations studied may operate internationally, the primary focus is domestic (Zaltman, Duncan, and Holbek 1973; Rowe et al. 1973; Van de Ven and Poole 1989; Hesselbein 2002). This orientation raises the concern over the universality and generalizability of the findings to other social settings. It also brings into question the models applicability to other cultures and governance systems.

The second point relates to the treatment of innovation in the public and private sphere. In many respects, there was little or no difference noted. While Zaltman, Duncan, and Holbek (1973) theorize a distinction, it primarily relates to the size of the decisionmaking body and the inclusive or exclusive nature of the goods or services. This distinction, moreover, mattered only in the initial implementation phase. In this phase, moreover, the individual innovators within the organization required some minimal amount of autonomy in order to operate.

The third point is that while many of the theorist took little note of the distinction between innovation in the public and private sphere they, nevertheless, postulate the potential universality of their theories (Rowe et al. 1973; Van de Ven, Angle, and Poole 1989). In this context, they assume innovation occurs in both sectors. Little attempt, however, was made to identify differences other than to acknowledge it was collectively complex. Finally, most theories were descriptive in nature and sought to understand and label this phenomenon. Thus, little progress in empirically testing the various theories has occurred. Walker (2010) posits this is primarily due to the complex and multifaceted nature of innovation. 
Bason (2010) addresses these three deficiencies in proposing a model of public sector innovation that represents a significant departure from past theorists. In contrast to earlier models, Bison's model is deliberately prescriptive rather than descriptive in nature. It derives from qualitative research methodologies. Bason (2010) also explores innovation outside of the United States. His research involved cases drawn from nations with longstanding and stable democratic traditions. Bason's (2010) exploration of innovation occurs within the context of transnational organizations (e.g. the European Commission). In addition to being politically similar, some of the nations are culturally congruent (e.g. Canada and Britain). Although this is by no means universally the case (e.g. Norway).

Bason's (2010) model posits that public sector innovation reflects an ecosystem composed of courage, consciousness, co-creation, and capacity. He operationalizes each, respectively, as leadership, awareness, process, and structure. The first attribute of his public sector ecosystem of innovation is the existence of a consciousness of an innovation landscape. This involves embedding a common understanding and awareness of the importance and function of innovation within the organization. In this regard, it closely aligns with the creation of an organizational culture of innovation (Schein 2004). Bason's model recognizes the existence of barriers that are in juxtaposition to the potential created by innovation.

Bason's (2010) model denotes some barriers to innovation, consisting of: lack of awareness, recognition, and reflection. His view is that the potential for innovation entails the opportunity to change culture through education, communication, and dialog. Capacity involves building innovation potential into the structure of the organization. In 
this regard barriers to, and potentials for, innovation exist in a strict hierarchy with political structure at the apex and the people and culture at the base. In the middle is, respectively, the strategy and the organization. At each level, it is necessary to understand the barriers to innovation in order to capitalize on the potential of innovation.

Unlike in previous models, Bason (2010) explicitly acknowledges the existence of co-creation. This is a process involving both the governing organization and its clients and citizens. Co-creation transpires within the context of a learning organization and occurs within a sequential process in which the ultimate goal is new learning. So while citizen involvement is important, so is design thinking in orchestrating, measuring, and learning within the framework of co-creation. Bason (2010) also considers the role of the strategic environment in cultivating innovation.

The final domain that the model addresses is leadership. Bason (2010) suggests the need to lead innovation at all levels. In this perspective, courage is the core requirement for visionary leadership. He frames leadership as the capacity to illuminate the innovation space and manage conflict. The latter presents a challenge because organizations build conflict into institutional structures (Morgan 2006). Bason (2010) posits the existence of a typology of leadership roles although it is unclear if an individual can simultaneously inhabit multiple roles without experiencing cognitive dissonance.

Culture is important to creating the conditions necessary for innovation (Schein 2004). There is also evidence that catalytic events provide the spark necessary for innovation (Van de Ven, Angle, and Poole 1989). Sahni, Wessel, and Christensen (2013), furthermore, posit that innovation cannot occur without the existence of certain enabling 
conditions. In accenting the twin attributes of change and performance inherent in innovation, Napier (2009) takes as his starting point that innovation is "change that outperforms previous practice" (30).

In this respect, Napier (2009) considers innovation as both a management function and as an environmental change. It is, nevertheless, clear that in either context there must exist a personal preference for risk-taking among the individuals involved in public sector innovation. This is the case since innovation is inherently risky. The research appears to suggest the necessity of creating a culture in which there is active celebration of experimentation, as opposed to mere passive protection (Walker 2010; Bason 2010). Risk and experimentation, therefore, appear central to spurring innovation.

Sahni, Wessel, and Christensen (2013) build on the work of earlier theorists to posit the existence of five conditions necessary for what they term "breakthrough innovation" (26). They view innovation through a public sector lens. In exploring the nature of change their research focuses on innovation at the municipal level. They argue that innovation breaks down along two dimensions. In the first dimension, the organization must possess the ability to both experiment and to "sunset outdated infrastructure" (28). These two conditions relate to the organic capabilities that individuals and organizations must possess. The second dimension includes the concurrent existence of feedback loops, incentives for product and service improvement, and budget constraints for end users. The latter three conditions point towards the need to consider the unique aspects inherent in public sector innovation. These revolve primarily around the issue of motivating collective actions in the public sector. Sahni, Wessel, and 
Christensen (2013) suggests a normative bent in theorizing about public sector innovation.

\section{Summary and Synthesis of the Literature at the Organizational Level}

This section proves a synthesis of the relevant literature. In recognizing the close link between innovation and implementation, the research of Zaltman, Duncan, and Holbek (1973) represents one of the important early contributions. Earlier theories tended to focus exclusively on innovation irrespective of other functions. This tended to oversimplify the subject and provided only limited utility. Another contribution was their formulation of a comprehensive list of attributes of innovation. This is one of the earliest attempts to provide a robust and holistic view of the phenomenon.

In characterizing the impact of each attribute on the process of innovation and implementation, they highlight the complex and multifaceted nature of this phenomenon. The themes that Zaltman, Duncan, and Holbek (1973) identified continue to resonate today. Their model, however, faces criticism that it is overly mechanistic. The model views innovation occurring in a linear fashion and each sub-stage serves as a link in a chain leading to innovation. This is problematic since Zaltman, Duncan, and Holbek (1973) provide a glimpse into the logic that shapes social life in a distinctive yet partial way. The manner in which they view organizations creates distortions that invites a more nuanced perspective (Morgan 2006). Subsequent theories on innovation took as their focus addressing these shortcomings (Rowe 1973).

One of the theorists who took on the challenge of addressing these weaknesses was Van de Ven (1989). His efforts to unravel this phenomenon resulted in a more 
comprehensive and nuanced understanding of innovation. The research program he led at the University of Minnesota spanned both the public and private sectors. This is a unique contribution since most theorists tend to focus on one sector to the exclusion of the other. His attempts at creating a theory-of-theories on innovation provided a comprehensive theoretical perspective in which his subject matter was explicitly the integration of various theories. The result was a metatheory.

In doing so, Van de Ven's (1989) research highlighted the complexity and interdependency of various approaches to understanding the phenomenon. Indeed, innovation, according to his model, is an iterative process that frequently results in restructuring not only the process itself but also the problem under consideration. His metatheory represents an ambitious attempt to reconcile often conflicting approaches to organizations, cognition, decision-making, and human behavior. Empirical evidence supporting the link between innovation and organizational performance, however, remains elusive (Walker 2010).

Bason (2010) subsequently explored the nature of public sector innovation in a variety of organizations outside of the United States. His research is prescriptive in nature and it was reliant on qualitative research methodologies. While this appears to meet the needs of practitioners, it also suggests the existence of inherent methodological limitations to developing, operationalizing, and empirically testing theories in this complex realm. In this framework, innovation is an iterative process that occurs within a dynamic environment (Bason 2010). Volatility, uncertainty, complexity, and ambiguity characterize this environment. 
One of the defining characteristics of Bason's (2010) approach was a focus on understanding and overcoming barriers to innovation through the creation of a common organizational culture. Morgan (2006) suggests that such an organizational culture "develops as an ethos created and sustained by social processes, images, symbols, and ritual" (128). In this context, innovation provides the framework for interpersonal interactions. Bason's (2010) research points to the need for public sector organizations to facilitate learning in order to transform themselves.

Theories on innovation universally assume a link exists between performance and innovation (Thompson 1973). The focus of research, therefore, is on both defining innovation and on understanding the various manifestations in which it appears. In the public sphere, this involves describing innovation in terms of both product and process (Goldsmith 2002). Technological innovation typically characterizes the former. The organizations strategy, structure, culture, and routinized behaviors are the result of processes (Rowe 1973).

The product of an organization, in this context, is either an inclusive or exclusive good or service. Each of the theories, furthermore, views the creation and diffusion of innovation as involving parties that are internal and external to the organization (Rogers 2003). The clear implication is that innovation spans various organizational echelons (Rogers 2003). Regardless of the type of innovation —or its source - the near universal baseline assumption is that it emerges from a perception that a gap exists in organizational performance (Segal 2006).

The pathway from the adoption decision to the final implementation of innovation is multilayered and complicated. Walker (2010) builds on the Van de Ven and Poole 
(1989) meta-theory of innovation. His major contribution is in reflecting this reality in his research. In surveying the field of public sector innovation, Walker (2010) notes the existence of four sets of observations regarding the relationship between innovation and performance. The first observation is the primacy of organizational characteristics in stimulating innovation.

The second observation is the implicit or explicit existence of a performance gap that the organization wishes to reduce or eliminate (Zaltman, Duncan, and Holbek 1973; Van de Ven, Angle, and Poole 1989). The third observation relates to the subject of the diffusion of innovation as the source of improving organizational performance (Rogers 2003). Researchers have only recently begun to consider the subject of the diffusion of innovation as a stand-alone topic. The final observation notes the link between performance and innovation from the perspective of typology. In this regard, Walker (2010) notes the existence of multiple performance goals that greatly complicate the organization's ability to define successful performance.

Walker (2010) notes that the lack of empirical studies calls into question the ability to make claims regarding both the measurement of innovation and the relationship between innovation and performance. For example, a question remains whether innovation leads to improved organizational success or organizational performance leads to innovation. The former would result in a focus on innovation while the latter would spur a concentration on improving organizational structure and building a supportive culture. Indeed, various researchers approach the study of organizations with a wide variety of academic and theoretical lenses. Each lens results in a different perspective on organizational performance. 
Walker (2010) also maintains that high levels of innovation may create organizational instability. This instability, Walker surmises, could lead to a degradation in individual and organizational performance. Walker identifies two longitudinal studies on the link between public sector innovation and performance. These studies are, however, limited to research conducted on one hundred US public libraries and over four hundred local governments in the United Kingdom. There is, moreover, a lapse of two decades between these sets of studies.

This limited dataset and the fact that Walker (2010) chooses to rely upon preexisting datasets to operationalize innovation and performance is a concern. Walker's (2010) overall findings infer that the adoption of innovation results in improving performance. So, he advanced a proposal for testing the innovation-performance hypothesis. In attempting to close the gap between theory and the corresponding lack of supporting data, Walker (2010) seeks to answer the question of whether the evidence warrants a decidedly pro-innovation bias in the research literature.

The research suggests that public sector leaders require many of the same management and leadership skills as private sector leaders (Zaltman, Duncan, and Holbek 1973; Shin and McClomb 1998). These include those traits associated with transformational leadership like visioning, inspiring, and risk-taking (Burns 1978). Given the diversity of public and private sector organizations, however, skill requirements vary greatly (Morgan et al. 2013). This reflects the contingent nature of organizational leadership. In this manner, leaders play a significant role in creating an organizational culture conducive to innovation regardless of the sector in which they operate (Jaskyte and Kisieliene 2006). 
Zaltman, Duncan, and Holbek (1973) argued that successful innovation requires the creation of an organizational climate in which it is possible to decentralize authority. This begins with leaders recognizing the need for organizational revitalization (Burns 1978). Tichy and Devanna (1986) suggest that it is possible to create a new vision of what innovation looks like by focusing the organization's attention on the future in a positive manner. They also imply the importance of institutionalizing innovation such that it survives the leader's tenure.

Leadership is a central feature of the co-production of public sector innovation. The leadership necessary to drive public sector innovation can emerge from the government or from the community (Lee, Hwang and Cho 2012). The research of Lee, Hwang, and Cho (2012) suggests leadership has multiple drivers. These differing sources of leadership, furthermore, reflect the existence of information asymmetry. There is, however, little in the literature regarding the role of information asymmetry in shaping public sector innovation. This gap is surprising considering the focus on information asymmetry in the field of political science and public administration.

This dissertations survey of the research on the co-production of public sector innovation revealed three general themes. These themes represent elements of a narrative that exhibit some level of universality or agreement between researchers in the literature. While existing theories of innovation touch on each of these themes to varying degrees, none provides a clear depiction of how they holistically relate to innovation. No theorist, moreover, has tied these three themes together in a meaningful or comprehensive manner under the rubric of public sector innovation. In other words, the dialytic linkages are missing or lack maturation between these three themes. 
The first of these themes is that institutions and organizations matter. They provide the setting and context for innovation. In this regard, institutions and organizations are a necessary, but not sufficient component, of public sector innovation. Leaders animate organizations towards a particular ends and have the power to either amplify or dampen the potency of innovation. Innovation requires leaders to reweave the social fabric of the organization. Leaders become social architects to the extent they are able to motivate individuals and exercise control over the bureaucracy.

The second theme that emerges from the literature is that leadership is critical to the co-production of public sector innovation. It is a necessary, but not sufficient, condition for innovation. Leadership can occur at any level of the organization. Likewise, innovation can arise at any level of the organization. Leadership is, moreover, highly contextual. It can manifest in any number of ways from transactional to transformational (Bass 1985). One constant theme in the literature is that leadership in the public sector has a normative component.

The final theme that emerges from the literature is the need for the active and often intimate involvement of customers or clients in public sector innovation. Customers and clients are a necessary, but not sufficient, component of public sector innovation. They play a central role in in the process of co-producing innovation. Co-production is process by which dynamic social interactions between groups result in a sharing of knowledge and the resources necessary to create public goods and services (Ostrom 1990). Ostrom (1990) reminds us that there is a uniquely social aspect to collective action. Customers and clients have the power to either advance or impede public sector innovation through the process of co-production. 


\section{Measuring Public Sector Innovation}

The measurement of innovation has taken on increasing salience for both academics and practitioners of innovation in recent years. The literature that is accumulating on the measurement of innovation ties it closely to the management of innovation (Adams, Bessant, and Phelps 2006). This reflects, to some degree, the emergence of the study of management as an independent field of academic specialization. In this regard, measurement is central to management (Aguilar 1988). Measurement extends to include management functions that relate to benchmarking and the process of making cross-organizational comparisons (Nagji and Tuff 2012). Measurement also provides a way of rewarding and awarding behavior that leads to innovation in such a manner as to encourage its continued practice.

Measurement requires an analytical framework (Ven, Angle, and Poole 1989; Adams, Bessant and Phelps 2006). This is a challenge, given the lack of any generally agreed-upon unifying theory of innovation. The result is a broad categorical approach to measurement. Adams, Bessant, and Phelps (2006) postulate that the literature on the measurement of innovation breaks down into seven categories, including: inputs, knowledge management, strategy, organization and culture, portfolio management, project management, and commercialization. This approach reflects an effort to measure all facets of innovation using multiple factors. The APSC (2011) authors claim this is necessary given the complex nature of innovation and the interdisciplinary approach to its study. 
The measurement of innovation at the organizational level often occurs in multiple dimensions. Most commonly, however, it involves elements of cost, quality, and time (Sahni, Wessel and Christensen 2013). Cost is typically, although not exclusively, viewed in economic terms. Monetization, however, is not always possible in the public sector where values often conflict (Morgan 2006; Gordon and Milakovich 1995). Monetization is also difficult when facing wicked problems involving complex, dynamic, and normative issues that are difficult to quantify. Therefore, for example, the linkages between environmental issues and normative or moral concerns surrounding quality of life are difficult to value in the traditional economic sense.

Qualitative measures of innovation involve subjective attributes or properties (Reese 1996). The question arises as to who determines the desirability of the attributes or properties. Quality represents the measure of benefits that accrue based on the attributes and properties of the good or service. In this regard, it is the role of the customer or client to define whether, and to what extent, quality exists in a good or service (Deming 1986). Often quality exists in relation to cost. The yardstick of quality is often manifest as a measure of value. Time is also subject to measurement. The quantification of time is typically an expression of lead-time. Lead-time is that temporal attribute which relates to the latency between the start and finish of a process. Lead-time represents, for example, the time between the initiation of a request and the complete execution of the process rendering a public good or service (Deming 1986).

The consideration of these three factors — cost, quality, and lead-time — often occurs in isolation and involves tradeoffs between them (Davis and Ingle 2013). This reflects an incremental perspective characteristic of a traditional maximizing 
methodology, as opposed to a more holistic and integrative approach. In traditional innovation, one or more of the elements improves while the other(s) remain constant or decrease by a comparatively small amount. Therefore, for example, it is possible to simultaneously experience a cost reduction, a slight lead-time increase, and hold quality constant. The ideal, however, is a simultaneous increase along all three dimensions. Davis and Ingle (2013) advise that when this is the case the proverbial innovation engine is hitting on all cylinders.

Recent efforts at measuring public sector innovation have also broken down along national lines (APSC 2011). The European Union's effort to create a European innovation scoreboard reflects this approach. The scoreboard represents a regional effort to analyze public sector innovation through the development of several innovation indicators. The goal of this initiative was to understand the various drivers and barriers to innovation (EU 2013). The scoreboard relies upon twenty-two quantitative and qualitative measures arrayed among three broad categories.

The APSC (2011) authors, however, do not weigh or aggregate the various measures so it is difficult to conclude their relative salience. This methodology has the effect of distorting the influence of various indications. The authors fail, moreover, to provide sufficient justification for the approach they took in reaching broad general conclusions across categories that are normative in nature. There is a risk, therefore, in generalizing their findings to the sub-national level. The APSC effort was descriptive in nature and did not suggest causal relationships.

Meyer and Goes (1988) are highly critical of nominal measurements. They assert that numerical quantification ignores the social context of innovation. This may well be 
the case; however, the authors narrow their research focus to an aggregate measure that ignores pre- and post-adoption events. This approach, therefore, fails to acknowledge the complex contextual milieu in which innovation occurs. There is also no assessment of the utility of specific innovations. Their ultimate effects are opaque and obscure.

\section{Innovation Below the Organizational Level}

A review of the literature suggests that four different levels of analysis exist in the study of innovation. They are the individual, group or team, organizational, and societal levels (King and Anderson 1995). These levels present a convenient way of bounding innovation theory. Each provides a unique lens through which to view this phenomenon. King and Anderson (1995) argue that these levels are artificial. They, nevertheless, conclude, "One of the most significant contributions which innovation research can offer to our understanding of organizational behavior is the possibility of integrating different levels of analysis" (King and Anderson 1995, 5).

The writings of King and Anderson (1995) illuminate the complex nature of innovation. In their view, differing levels interact to reveal a nuanced view of social interaction. Evaluating innovation along the continuum of levels offers the promise of a more holistic approach to understanding this phenomenon. It also presents a fundamental challenge to the social scientist attempting to model this phenomenon. There are limits to the cognitive capacities of the researcher such that they can only model and measure that which falls within their range of observation and comprehension (Dubin 1969).

The literature recommends two explanations for why the focus on innovation below the organizational level is necessary. The first is exemplified by West et al. (2003) 
who posit that among the reasons spurring research at the individual, work-group, and team level is the growing recognition that interventions at these points provide "the flexibility needed to respond effectively, appropriately, and quickly to the constantly changing demands in an organizations environment" (394). This suggests that it is possible to gain insight into innovation by disaggregating the organization and then studying its component parts.

Levitt and March (1988) illustrate the second explanation. They assert that the process of organizational learning inhibits innovation. The result of learning, they argue, is cognitive rigidity. This is the case since it is a challenge for individuals to change mental mindsets when confronted with contradictory information and instead attempt to bring the incongruous observation into conformity with existing mental models (Popper 1959). Put another way, the process of learning serves to reinforce an organizational commitment to the status quo (Hartley et al. 2013).

Burpitt and Bigoness (1977) propose that to overcome limiting factors research should focus on individuals, work-groups, and teams. The study of innovation below the organizational level that provides valuable insight into the process of innovation (West et al. 2003). A great deal of the literature on innovation at the sub-organizational level emerges from the fields of applied and behavioral psychology (King and Anderson 1995). This is in large measure the result of the prevalence of easily observable, measurable, and quantifiable individual behaviors at this level.

One possible conclusion is that the focus on research below the organizational level came about, in part, as a response to globalism. The premise is that the increase in international economic and military competition during the Second World War continued 
in the post-World War II era and subsequently spurred the need for quantifiable results to justify the allocation of scare economic resources. During this period, therefore, applied psychology held the promise of maximizing individual, group, or team contributions. Increasingly the view was that these contributions were essential to organizational success and institutional survival during this period (Porter 1990).

The research on innovation at the individual level emphasizes the empowerment of people and teams through self-actualization (West et al. 2003). Burpitt and Bigoness (1977) define empowerment as "any practice that enhances a belief in self-efficacy" and that "increases a sense of power" (415). Empowerment results in providing the team "freedom in developing solutions... providing early involvement in the initial planning stages of projects and participation throughout the life of the project" (Burpitt and Bigoness 1977, 422).

This reflects a social psychological approach to innovation. In this regard, intrinsic motivating factors inherent in the task itself, in addition to externally imposed personal rewards or punishments, drive innovative activity (King and Anderson 1995). In this perspective, greater allowances for freedom of action and the opportunity to increase personal knowledge and expertise provide the basis for engaging in innovation (Burpitt and Bigoness 1977). The motivation for action comes from within the individual such that self-actualization, for example, plays a role in fostering innovation.

At the individual level of analysis, creativity emerges in the literature as a salient factor in generating the conditions for innovation (West and Farr 1990; King and Anderson 1995; West 2002). King and Anderson (1995) acknowledge the difficulty of defining psychological concepts like creativity. They conceive of it in terms of persons, 
processes, and products. West (2002) defines creativity as the "development of ideas" and of "thinking new things" (356-357). There was little in the literature recommending financially contingent extrinsic rewards.

West (2002) goes on to posit a conceptual model of innovation composed of two components. The first is creativity. The role of creativity is to generate new ideas. The second component is implementation. West (2002) suggests this involves the organizational adoption of new ideas. In this regard, West (2002) defines innovation "as encompassing both stages - the development of ideas — creativity; followed by their application - the introduction of new and improved products, services, and ways of doing work" (357). West (2002) conceives of innovation, furthermore, as an iterative non-linear process.

Conversely, Rosenfeld and Servo (1990) posit a model of innovation that consists of conception, invention, and exploitation. In this context, “'conception' refers to any idea that is novel... 'Innovation' applies to any novel idea transformed into reality; and... 'Exploitation' normally implies wide acceptance and/or profitability” (252). They view innovation as a linear process that "almost always involves a prolonged battle amongst numerous people and that requires tremendous stamina and confidence on the part of the champion" (253). Their focus, therefore, is on optimizing the process of innovation. Rosenfeld and Servo (1990) also warn against conflating creativity and innovation. They view the former as a solitary starting point. It is, therefore, associated with conception.

Morgan (2006) observes that exogenous and endogenous turbulence and instability leads to greater autonomy and discretion in dealing with emergent organizational challenges. The result is adhocracy and the space for teams to flourish 
(Morgan 2006). The work-group or team serves to mediate between the individual and the larger organization of which they are a member. Scott (2003) defines a team as "groupings of personnel across departmental lines to carry on some portion of the regular work of the organization" (241).

There are a number of themes that appear in the literature on work-group or team innovation. They include leadership and the composition, structure, climate, and longevity of the group (King and Anderson 1995). The characteristics of each workgroup or team vary. Differing characteristics have a uniquely dynamic effect on innovation (Scott 2013). Research on innovation at the team and work-group level also emphasizes the opportunity to co-create with customers or clients (Burpitt and Bigoness 1977). The result of research into teams suggests that communication at this level is more personal, informal, and conversational. This runs counter to the Weberian perspective that prizes institutional formalization and routinization.

West (2002) identifies group tasks, diversity of knowledge and skills, and team integration as three prominent factors in achieving innovation at the work-group and team level. The group task represents the "fundamental influence on the work group, defining its structure, process, and functional requirements" (West 2002, 359). The diversity of knowledge and skills implies the need for creativity in problem solving. It also infers that innovation is a multistage process with different individual behaviors necessary depending on the situation (Scott and Bruce 1994). This does not imply, however, that innovation is a linear process (West 2002). Team integration suggests that the members are able to take advantage of their diverse skills and talents. The organization, however, continues to define the work-group, team, and individual routines. 
Leadership among and within teams and work-groups also has a prominent place in the literature. King (1990) advises that while there has been a significant amount of research on innovation and leadership, there is not yet any clear consensus on the exact nature and effect of leadership variables. West et al. (2003) advise that much of the research on team leadership has focused on the individual contribution of a single leader. This is because leadership "can also be provided by one or more individuals who are either formally appointed to the role or emerge from within the team" (West et al. 2003, 395). One of the central roles of leadership is in "developing team processes that facilitate innovation" (West et al. 2003, 394). This also highlights the important role of leadership in providing clarity around group tasks.

\section{Innovation Above the Organizational Level}

Theorists have advanced the concept of systems of innovation to account for imbalances in national economic performance (Lundvall 1988; Nelson 1993). This approach scales up innovation from the work group, division, institution, organization, and government level to a national political economy perspective. This theoretical framework, which has gained popularity in Europe, posits that innovation (both technological and organizational), institutional learning, and networks of social interactions are the analytical touchstones for understanding disparate national and regional economic performance. In this setting, the theoretical framework seeks to draw linkages among a national financial, political, legal, scientific, and technology institutional infrastructures to explain developmental outcomes. 
The definition of a system of innovation remains contested with a "wide variety of definitions" competing for preeminence (Oyelaran-Oyeyinka 2005, 7). Freeman (1987) advances the argument, regardless, that a national system of innovation consists of "the network of institutions in the public and private sectors whose activities and interactions initiate, import, modify, and diffuse new technologies” (2). Edquist (2001) asserts, furthermore, that innovation assumes the guise of an interactive learning process that occurs within an institutional context.

The salient unit of analysis for this model is a network of autonomous institutions and organizations operating within a state or region. In other words, the polity or national society emerges as the unit of analysis that various authors conceive of in terms of networks of institutions (Freeman 1987; Lundvall 1988; Nelson 1993). One possible origin of this line of reasoning is the logic of collective action. Olson (1971) suggests that coercive state based actions are necessary to create organizational outcomes on a large scale.

Systems innovation attempts to reframe the concept of innovation in a manner that moves the conception of innovation to levels above the organizational, departmental, team, or individual levels. The conceptual framework, however, subsumes these levels within the network of autonomous institutions and organizations. This perspective, moreover, reflects a broad social aggregation. As an emerging conceptual framework, rooted in political economy, the systems of innovation approach benefits from an international relations (IR) perspective.

The systems of innovation perspective considers the nation, and not necessarily the state, when analyzing political and public policy outcomes (Porter 1990). This 
suggests the social, rather than political, nature of this perspective. While rejecting dependency theory, the systems of innovation approach appears to be offering a competing narrative to world-systems theory (Wallerstein 1974). It is worth pointing out that one of the early proponents of the systems of innovation approach to explaining economic and political outcomes was the United Nations.

Central to the systems of innovation theory is the suggestion that "the elements and relationships which interact in the production, diffusion and use of new, and economically useful, knowledge... are either located within or rooted inside the borders of a nation state" (Lundvall 1992, 12). This approach suggests that national economic capacity building is an inherently complex process. Integral in this perspective is the need for a robust network of institutions. They represent the ante for economic and social prosperity.

The systems of innovation approach also appears to recommend against traditional developmental policies involving technology transfer. National or regional networks are successful; moreover, to the extent they are capable of modulating uncertainty, conflict, and the flow of information (Oyelaran-Oyeyinka 2005). Lundvall's (1992) approach to the systems of innovation approach, however, has the effect of creating one economy for knowledge acquisition and dissemination — or innovation — and another for commercial endeavors.

Innovation in this context represents the endogenous dynamism of the underlying institutional forms and practices necessary to foster the "creation, validation and distribution of learning and knowledge, which are prerequisites of economic change" (Oyelaran-Oyeyinka 2005, 9). According to this view, path-dependent institutions shape 
the social processes in which innovation imbeds. This approach suggests that specific national or sectorial factors, which determine international economic competitiveness, are themselves the result of national institutions. Economic capacity, therefore, imbeds within national institutions.

Institutions can be endogenous in nature or the exogenous result of a colonial or Western heritage (Oyelaran-Oyeyinka 2005). The system of innovation framework is a more or less static explanation of existing social and economic performance. It does not provide a robust explanation of the political change necessary to foster future development or the capacity to govern (Fukuyama 2015). It also does not explain the nature of change over time. This lack of dynamism presents a serious limitation to this theoretical approach.

\section{Leadership, Organizational Culture, and Innovation}

The research suggests that the ability of a leader to foster innovation stems from social influence and not from formal power or authority (Goleman 2000). The exercise of leadership is not a proverbial individual sport, and invariably requires others. Leadership styles can and should differ depending on circumstances (Northhouse 2004). Recently the literature has begun to stress the need to consider the cognitive characteristics of leadership. In this regard, Goleman, Boyatzis, and McKee (2002) assert that leadership is dependent on emotional intelligence. They define emotional intelligence as the ability to manage oneself and one's relationship with others.

Several themes animate the literature on leadership and innovation. They emerge from differing perspectives on leadership that researchers have adopted. Reuvers et al 
(2008) posit that, "Leadership has been studied in various ways, depending on the researchers' methodological preferences and definitions of leadership" (229). The research suggests, moreover, that the ability of a leader to foster innovation stems from social influence and not from formal power or authority (Goleman 2000) — there is a wider social and emotional aspect to leadership (Scott 2003).

The leader's efficiency and effectiveness in maximizing the effort of others nevertheless varies (Goleman 2000; Ericsson, Prietela, and Cokely 2007). From this perspective, there is no single path to effective leadership. Organizations exist as social systems. They differ from other social systems in the extent they focus on setting goals and mobilizing resources towards goal achievement (Scott 2003). The leader, furthermore, must have a vision for the organization. This represents an idea of what the organization should accomplish. In this context, the influence of the leader is in achieving a deliberately intended outcome (Collins 2005).

The leader must have a vision of what they would like the organization to accomplish (Bass 1985). A vision refers to an idea or notion about the future state of the organization. In this regard, the influence of the leader has a deliberate and intentional outcome (Collins 2005). Researches also posit that leadership styles can and should differ depending on circumstances the leader faces (Northouse 2004). The efficiency and effectiveness of leadership styles in maximizing the efforts of others nevertheless varies (Goleman 2000).

From this perspective, there is no single path to effective organizational leadership. Integrating the various themes into a coherent approach to leading innovation is critical for practitioners and theorist alike. With so many organizations asking their 
best employees to be both leaders and managers, there is an inherent danger of organizational dysfunction. This occurs through the repeated leveraging of competing requirements for leading innovation and managing change. To address this concern researchers have focused on leadership — as it relates to innovation—separate and apart from management—which stresses incremental improvements.

This dissertation echoes this theme and maintains a focus on leadership. Management roles and responsibilities related to innovation remains peripheral to this dissertation. This is the case since the role of manager is to seek stability and predictability. Their focus, therefore, is on the incremental improvement of existing processes and products. Leaders, on the other hand, opt for the turbulence of innovation in order to move the organization forward. The roles and functions of managers and leaders exist in diametrical opposition (Vaccaro 2012).

Two streams of research have emerged on leadership and innovation (Ekenkov, Judge, and Wright 2005). The first stream stresses a full range of leadership. It represents a synthesis of various approaches and is manifest in the seminal work of Bass (1985). Beginning in the late 1980s, his theory of transformational leadership, along with the competing theory of charismatic leadership, has come to dominant the literature. Theories on leadership charisma predate the work of Bass (1985). Charisma theory focuses on the innate ability of leaders to inspire and motivate followers towards high levels of commitment to an organization or the organizations mission (Morgan 2006).

The theory that Bass (1985) proposes takes a broad view of leadership. According to Bass' (1985) theory, leadership manifests in two differing forms. The forms exist in a dialectic relationship. The first form is transactional leadership. This form of leadership 
reflects an exchange whereby the emphasis is on the behaviors, activities, and attitudes of individuals in leadership and followership roles. Management is largely by exception and rewards are contingent on behavioral outcomes. The focus is on shorter-term tactical adjustments necessary to deal with immediate environmental challenges.

The second form is transformational in nature. In this form of leadership, the emphasis is on the leader's effect on followers. This includes such factors as the leader's ability to build a relationship of trust and confidence with followers (Bass 1985). It involves creating a shared vision of the future and then motivating individuals to accomplish the vision (Tichy and Devanna 1990). The focus is on long-term structural change within the organization necessary to take advantage of opportunities in their strategic environment.

While transactional and transformational leadership are distinct forms, they do not necessarily represent mutually exclusive manifestations of leadership. Reuvers et al. (2008) posit that transactional leadership serves as a base from which transformational leadership can extend or expand. Bass (1985) recommends that an important requirement of effective leadership is the judicious employment of both the transactional and transformational forms. He holds that the form of leadership—-transactional or transformation - that an individual may choose to exercise is dependent on the situation faced. The utility of transactional and transformational leadership to this dissertation stems in part from its manifestation at the organizational level (Bass 1985). In this context, it is a contingent form of leadership.

Research suggests that a strong theoretical foundation exists for concluding that there is a positive relationship between transformational leadership and organizational 
innovation (Jung et al. 2003; Reuvers et al. 2008). Hartley et al. (2013) posit this is the case because it "strengthens strategic responsibility for creating substantial organizational change (including innovation)" (825). Transformational leadership begins with the recognition of the need to make a fundamental change and it ends with the institutionalization of organizational change (Tichy and Devanna 1990). It involves creating a vision, mobilizing the organization, motivating individuals, and reweaving the social fabric of the organization. These actions are central to innovation at the organizational level (Elenkov 2005).

The empirical evidence supports the argument that a positive relationship exists between transformation leadership and organizational innovation (Gumusluoglu and Ilsev 2009; Hartley et al. 2013). The evidence suggests, however, that this occurs in part because of the mediating effect of support for innovation (Jung et al. 2003; Reuvers et al. 2008). There is some disagreement, however, on the salience of support-which is internal or external to the firm. The transformational form of leadership, moreover, serves to facilitate a diverse set of management tasks inherent in innovation. They include formulating effective operational and developmental strategies, making decisions that address complex challenges and opportunities, and helping individuals through the anxiety and trauma of the unfamiliar.

The second stream of leadership research emphasizes the central role of vision and reflects the narrow view of leadership with respect to innovation. The leader creates an image of organizational processes and outcomes that serves as the basis for innovation (Elenkov el al. 2005). In this context, "visionary leadership refers to the ability to create and articulate a realistic, credible, and attractive vision of the future for an organization or 
organizational unit" (668). Shin and McClomb (1998) propose that vision-setting leaders employ "an inventive, risk-taking style" (5). From this perspective vision setting, leadership behavior eclipses other more supportive or goal directed styles when it comes to driving organizational innovation.

The implication of this research stream is that it is possible to crosswalk the same leadership behaviors between the public and private sector in order to facilitate innovation (Shin and McClomb 1998). In this paradigm innovation is linked to strategic leadership which Elenkov et al. (2005) define as "the process of forming a vision for the future, communicating it to subordinates, stimulating and motivating followers, and engaging in strategy-support exchanges with peers and subordinates" (666). Elenkov et al. (2005) conclude that the presence of strategic leadership promotes organizational innovation. Shin and McClomb (1998) hold this is the case since leaders exhibiting this style are better suited for the challenges of organizational innovation than are leaders demonstrating other styles.

Regardless of the leadership stream, the subject of organizational culture remains closely allied with leadership. Morgan (2006) touches upon this when he states that, "effective organizational change implies cultural change" (138). Since innovation represents change, it is possible to expect a shift in organizational values, norms, beliefs and assumptions to accompany innovation. This has important organizational implications. Leaders, moreover, have a uniquely salient influence on organizational culture. They can use this influence to create the necessary conditions for innovation (Schein 2004). 
King and Anderson (1995) posit that understanding organizational culture will "take us further in making sense of innovation... than a focus solely on more tangible aspects such as leadership styles" (106). In this regard organizational culture plays an important, if not critical, role in supporting innovation. Jassawalla and Sashittal (2002) advise that organizational cultures that embrace the norms of collaboration, creativity, and risk-taking also enjoy highly efficient and effective new product development processes.

Veiseh (2014) asserts there is a "meaningful relation between transformational leadership and organizational culture" such that it is "affected by factors such as hopeful influence, inspirational motivation and personal observations" (113). Porter (1990) concludes that culture plays a necessary, but not sufficient, role in fostering innovation. Jaskyte and Dressler (2008) argue, regardless, for the "inclusion of organizational culture in models of innovation" (35). Jaskyte and Kisieliene (2006) assert the existence of a positive relationship between organizational innovation, organizational culture, and leadership.

\section{Public Sector Co-Production}

The concept of co-production provides a theoretical framework for defining the relationship between the economy and society. Olson (1965) posits that in the absence of coercion, the provision of common public goods and services becomes less and less optimal as the size of the community increases. Ostrom (1996) counters this orthodoxy by suggesting that it is nevertheless possible to increase the level of public goods and services, and thereby provide higher levels of social benefits, through co-production. Co- 
production seeks, therefore, to describe the process or mechanism by which individuals and organizations mobilize to provide public goods and services.

Co-production aligns with the emerging New Public Governance paradigm. This is the case given the emphasis on service processes and outcomes. Citizens participate in governance as active agents of social change. In this regard, "trust and relational contracts" defines the governance mechanism (Osborne 2006, 383). Co-production is a relationship-based conceptualization of public governance. Ostrom (1996) concludes that where activities are co-produced, both services and communities are effective agents of social, political, and economic change.

Co-production is rooted in the political economy concept of a core social economy. Boyle and Harris (2009) identify the core economy as representing the "social economy of family and neighborhood" (5). Co-production involves social units acting in a manner that creates value for those units. In this perspective, co-production is the underlying operating system within society. It represents the resources and relationships necessary for the generation and delivery of public goods and services (Boyle and Harris 2009). Together, resources and relationships compose the core economy.

Resources, in the traditional political economy sense, are those inputs that society uses to produce outputs. In this context, outputs represent public goods and services. Resources make up the first critical component of the core economy. They can include conventional inputs such as labor, capital, and land. Resources can also include the political and social capital necessary to operate on behalf of others. In co-production, one critical source of resources is the organization's clients. In this instance, resources embed in the everyday life of individuals. 
The concept of co-production presents a nuanced view of resources. In the context of co-production, they can include, for example, the time and energy a client is willing to invest in co-production. Likewise, the client may possess unique knowledge or experience related to a particular policy domain. Ostrom (1990) argues this is the case since clients are an essential source of insider information that local service providers depend upon. Finally, clients possess important skills and talents useful to the organization and their leaders.

Relationships represent the second critical component of the core economy. Relationships find expression through individual behavior. One important social relationship is between the customer or client and the service professional. The latter often identifies with their functional specialization. Co-production occurs through thick relationships rather than through thin market transactions (Ostrom 1990). Relationships are central to the creation and ongoing maintenance of social networks. They represent the type and manner of social connection between individuals or groups of individuals.

Therefore, for example, individuals can forge connections, links, and bonds based on a socially constructed identity. These social connections can manifest at the emotional level through feelings of compassion, empathy, or tolerance (Ostrom 1996).

Relationships reflect the way two or more individuals regard and behave towards each other (Morgan 2006). Key to co-production is the continual maintenance of a respectful and supportive relationship between the client and service-providing professional (Alford 1998).

Public goods and services are artifacts of the resources and relationships that animate the core economy. If the core economy represents the allegorical operating 
system of a computer, the organizational routines that result in public goods and services represent the metaphorical computer programs (Boyle and Harris 2009). In this perspective, collective action becomes the mechanism for creating specialized goods and services (Olson 1971). In other words, co-production involves individuals mobilizing the core economy to produce socially constructed goods and services.

The central idea of co-production is that individuals who consume public goods and services are the source of the resources and relationships necessary for the efficient and effective generation and delivery of those same goods and services (Boyle and Harris 2009). Co-production, therefore, invites us to focus on human resources in our efforts to "remake the social networks that reduce demand on professionals and support public service interventions" (Boyle and Harris 2009, 11). Human resources reside within the knowledge, motivation, skills, and life experiences of individual social actors.

The goal of co-production is to increase the marginal utility of public sector goods and services for clients of public sector organizations (Ostrom 1990). While coproduction plays a role in general-purpose governance, it is most reflective in the special district governance typology. The emphasis of special districts on the provision of a single or limited number of pubic goods or services enables clients to concentrate their resources on regulating special district output (Foster 1997). The client's focus is on modulating the marginal utility of goods or services in a manner that maximizes their utility.

Alford (1998) posits that a difference exists in the various roles of citizen, client, and volunteer in public sector activities. The typology he advances provides a more descriptive explanation of the incentives of individuals involved in co-production. In this 
regard "volunteers are analogous to the suppliers of inputs to the firm" and "clients are analogous to its buyers" (Alford 1998, 129). Roberts (2004) opines that it is the role of citizens to serve as volunteers and clients in a democratic society. This is an important distinction since some theories of co-production conflate these roles or fail to address them in a meaningful manner.

Co-production complements the philosophy of the New Public Governance movement by focusing on the relationship between public sector organizations and the for-profit or private sector individuals they serve. New Public Management, alternatively, characterizes the latter as preferred suppliers. Normann (1984) asserts that as the user of services, clients are competent and cognizant of the benefits of active participation in the development and delivery of common public goods and services. Co-production serves to connect individuals and, thereby, harmonize the process of governance (Ostrom 1990).

In the context of the New Public Governance movement, the co-production relationship is one of interdependent agents operating within ongoing institutionalized relationships. Governance becomes, therefore, a shared responsibility. Alford (1998) goes on to argue, "Value cannot be created or delivered unless the client actively contributes to its creation" (130). Individual agency, however, is both constrained and restrained by institutional factors. In this regard, the lack of intra-organizational competition and the unitary focus of special districts benefits the organization's customers and clients (Burns 1994).

This is in part because there is a limited potential for competition in the provision of alternative goods and services. This is largely due to the absence of alternative general-purpose or special district governing organizations. Client co-production is, 
therefore, vital to the creation and delivery of public goods and services. In this circumstance, co-production represents "an integrating mechanism and an incentive for resource mobilization" (Bovaird 2007, 846). There is, nevertheless, a vital role for government to play in insuring equity.

Co-production represents an important point of departure for restoring trust in public institutions. Levine and Fisher (1984) argue that public sector reform that relies on marketization for the delivery of pubic goods and services undercuts the core economy. This is due to an overreliance on contracting. Contracting represents a thin relationship that focuses narrowly on economic outcomes as performance criterion. The alternate view is that contracts are the manifestation of social values that imbed in institutions at the time of their formation (Cooper 2003). Contracting assumes the adjudication of value conflicts will occur through the legal systems.

Co-production assumes the adjudication of values conflicts will occur through the political process. Co-production is a normative process. It seeks to infuse socially constructed values into the creation and delivery of pubic goods and services. It is, therefore, at the heart of the New Public Governance paradigm. This movement emerged in response to concerns over the market orientation of New Public Management. In this regard, co-production seeks more than a voice for individuals to contribute their ideas. It suggests that society can only hope to achieve optimal levels of public goods and services though the active participation of the organization's clients.

Co-production raises important questions regarding power, control, and equity. It also holds the potential of reducing fiscal stress through the devolution of service responsibilities (Levine and Fisher 1984). Co-production is not an attempt to create a 
bridge to the withdrawal of services. It is instead an effort to improve the utility of public goods and services. Likewise, co-production should not allow resource-rich communities to benefit at the expense of resource-poor communities. While goods and services may appear different in differing communities, co-production represents a beneficial tailoring to local needs (Ostrom 1990).

Brudney and English (1983) theorize that passivity on the part of the client does not necessarily negate co-production. They suggest instead that inactivity indicates the existence of a typology of co-production that includes both active and passive components. Ferris (1984) recommends, furthermore, a modification of the concept of co-production to recognize the central role of revenue generation. He introduces the term co-provision to describe the voluntary acquiescence to public financing. This includes the exercise of taxing authority, utilization of the power of emanate domain, and the ability to issue public debt. Ferris (1984) concludes that co-provision is central to improving efficiency and equity.

\section{The Origins of Special Districts}

A special district, in the simplest form, represents a unit of local government created by an act of a state's legislature to perform certain delimited functions. At last count, a little over 35,000 special districts exist in the United States (Census 2002). This number is exclusive of school districts. Special districts represent roughly forty percent of the total number of local governments in the United States. Their growth in numbers, moreover, continues unabated while the number of municipalities, townships, counties, and independent school districts remains relatively stagnant (Census 2002). 
As narrowly focused, and nominally independent, professionally run organizations special districts reflect the zenith of the New Public Management movement. Governmental institutions are inherently normative and exist as artifacts of the fundamental beliefs and values of the society in which they embed (Morgan 2006). The coercive nature of governmental institutions, furthermore, provides a means though which a society can moderate conflicting values. In this perspective, special districts reflect an attempt to mediate the inherent tension between public and private interests (Porter et al. 1987).

It is through the canonical contributions of Burns (1994) and Foster (1997) that we have largely come to know the structure, function, and history of special districts. Burns (1994) observes that there have been more local American governments formed "in the twentieth century than when settling the West or the Midwest — or the East" (4). She remarks, "In the last fifty years, the large majority of these new governments have been special districts, not cities" (Burns 1994, 4). She concludes that new general and special-purpose governments resulted from a desire for purposeful racial exclusion (in the 1950s and 1960s), lower taxes (in the 1960s, 1970s, and 1980s), and new levels of public goods (in the 1950s and 1960s). Her central point is that institutional choices shape social outcomes in America.

Foster (1997) provides a slightly different take on the proliferation of special districts. She concludes that, "Special districts satisfy many needs from many different interest groups" (Forster 1997, 231). This contrasts with Burns' (1994) contention that special districts primarily serve the interest of landowners and real estate developers. Another topic over which they disagree is the fiscal impact of special districts. Foster 
(1997) calculates that metropolitan areas that rely on special districts for service delivery spend more per capita than do similar areas in which special districts are relatively nonexistent.

Foster (1997) asserts, moreover, that an upward spending bias exists over time in which special district costs grow at a rate distortional to that of general economic growth. This is inconsistent with Burns (1994) who posits that higher taxes—especially corporate taxes - should drive the formation of new cities. One can infer from these conclusions that the proverbial sun should be setting on special districts, considering their contribution to profligate public spending. This ought to be the case since citizens and politicians alike have a stake in quashing runaway organizational spending. Special districts, however, continue to thrive.

Burns (1994) posits that the reasons and resources available for government formation vary over time. For example, states empowered municipalities to create exclusionary zoning ordinances and to employ eminent domain after the Supreme Court struck down restrictive covenants. Burns (1994) posits, furthermore, that where the federal "Voting Rights Act preclearance requirements are in effect, the supply of all types of local governments is lower" (129). Burns (1994) further posits that private parochial interests can constrain governing institutions, if they so choose, by either abandoning them or simply by creating alternatives.

Burns (1994) recommends that private parochial interests have the ability to change government over time. This is the case since the degree of political participation and representation varies depending on the structural characteristics of local institutions. In the case of special districts, she argues that this privileges real estate developers. Burns 
(1994) also suggests that special districts serve to embed private market values in local public institutions. In doing so, she makes a normative recommendation for purging private values from public institutions.

Burns (1994) conceives of local governments as bundles of particular institutions, which states allow, and the federal government conditions. Her research illustrates how institutions grant the players political and economic power through control over taxing, zoning, debt, eminent domain, and service levels. She concluded this has four implications for governance. The first revolved around institutional definition in which the reasons for local government formation vary over time. The second is that businesses and developers can constrain governing institutions if they so choose. The third is that political actors have the ability to change government over time. The fourth is the embedding of private market values in local public institutions.

Burns (1994) asserts that, "Institutions of local governments are the laws created by state legislatures and sometime modified by the federal government that define the powers of local governments" (8). These "local governments... are bundles of particular institutions" that modulate social choice though the exercise of their powers (Burns 1994, 8). She posits that the various ways in which states allow political entrepreneurs to bundle these institutions, and the resulting manifestations of power at the local level, modulates social outcomes. She advocates from the perspective that various political entrepreneurs affect the local bundling of institutions to varying degrees. Successful political entrepreneurs then institutionalize their values in local governments.

Burns (1994) defines special districts as "independent local governments that generally perform only a few local government functions" (8). She goes on, however, to 
exclude school districts from this definition. The ubiquity of school districts leads to their disproportionally large effect on the results of special district research (Foster 1997). Without separating them out and segregating the data, all special districts tend to resemble school districts. Burns (1994) views the consequences of institutional choice in terms of powers, services, financing, accountability, and participation. Her research suggests that the first three relate to the structure of government while the latter two reflect the extent to which institutional choices reflect democratic values.

Burns (1994) conducts a longitudinal study of two-hundred randomly selected US counties to discern the factors leading to the formation of general and special-purpose governments. Her study covers thirty-seven years beginning in 1950 and she analyzes four separate periods: the 1950s, 1960s, 1970s, and 1980s. The unit of analysis is the county. She relies mainly on readily available US Census data for these periods. There are, however, gaps in this secondary source. This approach, for example, does not allow for data specifically tailored to her research question.

Burn (1994) uses the creation of general or special-purpose governments as the dependent variable. The author relies upon sixteen variables to infer five operative concepts that influence the creation of new cities and special districts. In this manner, Burns (1994) is able to operationalize the concept of special districts. The five conceptual components of her argument are race, services, taxes, supply, and entrepreneurs/small groups. These conceptual components represent mental images or perceptions regarding those things that create the conditions for special districts.

Burns (1994) asserts that political entrepreneurs embed private values in public institutions. As the author points out, this has implications for democratic governance. 
She argues that since special districts represent the private values of privileged developers they are ipso facto unrepresentative and unaccountable by nature. The counter argument, which the author fails to consider, is that policy decisions rarely deviate from the majority's essential preferences (Dahl 1956). This is the case since both citizens and their representatives share a consensus on important values. Dahl (1956) comments that with "such a consensus the disputes over policy alternatives are nearly always disputes over a set of alternatives that have already been winnowed down to those within the broad area of basic agreement" (131-132). The counter-counter argument is that special districts may not consider the majority's views because of their "hidden" nature and the potential for hijacking by business entrepreneurs.

Burns (1994) builds on the work of Foster (1997). While both authors take a qualitative approach and both rely upon US Bureau of Census data, they consider the study of special districts from two different perspectives. Burns (1994) takes a postpositivist approach to describe inductively the emergence of special districts. She appears to be attempting to build a theory. Although in the end, she chooses to avoid just such a pronouncement. She nevertheless postulates the causal mechanisms by which special districts form.

On the other hand, Foster (1997) takes a positivist approach in which she proposes a theory explaining the emergence of special districts and then tests her theory. In doing so, she develops and tests several hypothesizes. This reflects a realist view in which theories exist as true or false statements (Dubin 1969). Along the way, Foster (1997) considers and then rejects four alternative explanations for the emergence of 
special districts: institutional reform, public choice, metropolitan ecology, and structuralism. The result is a nuanced view of special districts.

Special districts are inherently normative organizations because they represent the institutionalization of values. The critical question, however, is: "Whose values?" At the heart of democratic theory resides the question of who benefits from government action and how. In this environment, the actions of special-purpose governments result in either the concentration or diffusion of costs and benefits throughout the district's jurisdiction. In this regard, the revenue that a special district receives signals the policy preferences of citizens. The goods or services the district provides represent those policy outcomes for which citizens will hold special district leadership accountable (Manin et al. 1999).

\section{Special Districts as Empirical Settings}

Special districts provide the setting for this study. As such, it is not only necessary to understand their function but also their form. In this regard, size often dictates form and special districts come in a variety of different sizes, which effects their shape. Indeed, no generic blueprint exists and each is unique in form if not specific function. Very small special districts may have no full-time staff and a small budget that requires the governing board to act as staff (SDAO 2013). The organization's operations are ad hoc in nature with little formal recordkeeping, standardization, or automation of key functions. Very small districts may lack standard written procedures necessary to routinize practices. Small organizations, on the other hand, generally have full-time managers but no professional support staff (SDAO 2013). Managers perform both line and staff functions. Associations and informal peer networks typically advise small organizations. 
In a medium-sized special district, professional staff support organizational leaders (SDAO 2013). Managers and staff may have responsibility for multiple functional areas (for example, the combination of accounting, administration, and human resources under a single manager is common in the authors' experience) and there is a delineation and specialization of functions. Larger budgets mean that these organizations can invest in professional training and consultative services. Large organizations maintain a fulltime professional staff for major functional areas (SDAO 2013). They engage in complex operations requiring ongoing planning. Large organizations typically have formalized policies and procedures.

Camison-Zornoza et al. (2004) assert, “The literature offers contradictory findings about the direction and the intensity of the relationship between size and innovation" (332). Damanpour (1992) argues, for example, that there is a positive relationship between innovation and size. This reflects the greater resources available to the organization for innovation. According to this view, the larger the organization the greater the disposition towards innovation. Hage (1980) offers a counterpoint in positing that the relationship is negative. According to this perspective, smaller organizations are more innovative. This is a reflection; the argument goes, of organizational flexibility.

The nature of special districts relates the types of goods and services they provide. Some special districts provide inclusive common goods while others provide exclusive public goods. The US Census Bureau conducts a census of governments every five years. This is perhaps the best source of information on special districts. The census of governments results in a universal "list of governmental units, classified according to type of government" which "includes tabulations... by state, type of government, size 
and county location" (v). The Bureau classifies special districts according to their functional orientation (Census 2002). This generally equates to the type of service they provide. Table 1 outlines the various categories, sub-categories, and classifications of special districts that the Bureau uses. The Bureau also delineates between single- and multiple-function districts.

Table 1. Special District Functional Classifications

\begin{tabular}{|c|c|c|c|c|c|}
\hline \multirow{2}{*}{$\begin{array}{l}\text { Function } \\
\text { Single }\end{array}$} & \multirow{2}{*}{$\begin{array}{l}\text { Categories } \\
\text { Education } \\
\end{array}$} & \multirow{2}{*}{$\begin{array}{l}\text { Sub- } \\
\text { categories }\end{array}$} & \multicolumn{3}{|l|}{ Classifications } \\
\hline & & & Education & Libraries & \\
\hline & Social & & Hospitals & Health & Welfare \\
\hline & Transportation & & Highways & Air & Other \\
\hline & \multirow[t]{2}{*}{$\begin{array}{l}\text { Environmental } \\
\text { and Housing }\end{array}$} & $\begin{array}{l}\text { Natural } \\
\text { Resources }\end{array}$ & $\begin{array}{l}\text { Drainage and } \\
\text { Flood Control }\end{array}$ & $\begin{array}{l}\text { Soil and } \\
\text { Water } \\
\text { Conservation }\end{array}$ & Other \\
\hline & & & $\begin{array}{l}\text { Parks and } \\
\text { Recreation }\end{array}$ & $\begin{array}{l}\text { Housing and } \\
\text { Community } \\
\text { Development }\end{array}$ & $\begin{array}{l}\text { Sewage and } \\
\text { Solid Waste }\end{array}$ \\
\hline & Utilities & & Water Supply & Other & \\
\hline & Fire & & & & \\
\hline & Cemeteries & & & & \\
\hline & $\begin{array}{l}\text { Industrial } \\
\text { Development }\end{array}$ & & & & \\
\hline Multiple & & & $\begin{array}{l}\text { Natural } \\
\text { Resources and } \\
\text { Water Supply }\end{array}$ & $\begin{array}{l}\text { Sewage and } \\
\text { Water Supply }\end{array}$ & \\
\hline
\end{tabular}

(Source: Census Bureau, 2002)

Given their diversity, classifying special districts is an inherently challenging task. Therefore, for example, although the Bureau specifically excludes school districts from its definition of special districts it does include authorities created for financing school infrastructure. It refers to those as educational special districts (Census 2002, 14). Another example of the challenge of neatly categorizing special districts is the "Other" 108 
utilities classification in which the Bureau includes electric power, gas supply, and public transit.

Similarly, there is a great deal of overlap within the natural resources domain such that the Bureau created a unique sub-category. This was not enough, however, to contain the various natural resource functions. The result is such that irrigation, reclamation, and natural resource functions carried out by special districts are often classified elsewhere (Census 2002, 14). This approach to bucketing the data is a source of confusion. It also raises a question of when does a multiple function special district become a general form of government.

Both Burns (1994) and Bauroth (2010) posit that changes in service demands drive special district formation. Foster (1997) indicates that public and political entrepreneurs signal their preferences at the inception of the special district by institutionalizing a specific set of values in the enabling legislation. Routinized institutional modalities provide the mechanism for values attainment (Bauroth 2010). Ostrom, Gardner, and Walker (1994) advise, for example, that institutions like special districts represent local agreements over the management of common pool resources. In this regard, non-market public choice decision-making results from trust, communication, and reciprocity. The result is the creation of a shared community. The special district is an artifact of this political and economic outcome.

Most of the over 35,000 special districts are involved in providing inclusive public goods and services like public health, public transportation, or the protection of natural resources. Local general-purpose governments face limitations on their power, which often makes special districts an attractive alternative for delivering government 
services. This is particularly so where limits on the ability of general-purpose governments to raise revenue through taxation or the assumption of debt (Foster 1997). Limitations - fiscal or otherwise - predominately emanate from the state. Federal limitations, however, also exist.

General- and special-purpose governments are creatures of the state. They exist at the sole discretion of the state in which they reside and can only exercise that power which the state explicitly grants (Burns 1994). The federal government, particularly the judicial branch, can also impose restraints such as prohibiting certain actions as discriminatory. This federalized arrangement insures the maintenance of vertical equity such that uniform legal standards exist. The latter serves to maintain national social and political cohesion. The federalized arrangement also provides some measure of accountability, although it is indirect at best.

The large number and wide variety of special districts results from two principle factors. The first is that certain states favor special districts, and where this is the case, their numbers proliferate (Census 2002; Bauroth 2009). Illustrative of this factor is that over forty percent of special districts exist in just eleven states. The second factor explaining the great variety of special districts is their single-purpose nature. This requires more entities than if the public goods and services were provided by a single general-purpose government. Over ninety percent of special districts are single purpose entities (Census 2002).

The various categories and sub-categories of government service have the potential to call forth yet another dedicated special district (Bauroth 2009). A case in point is Multnomah County in Oregon. In this county, forty-two separate governments 
exist. The number rises to forty-four if State and Federal governments are included. Special districts make up twenty-four of the county's governments. Each special-purpose district, moreover, has a jurisdiction that may overlap that of the six cities and eleven school districts in Multnomah County.

The proliferation and overlapping jurisdictions of special districts presents a number of challenges. One challenge is that the large number of special districts makes it difficult to coordinate the activities of local service providers (Bollens 1986). This can include organizations in the private, public, and non-profit sectors providing the same services to similar clients. This can occur along either geographical or functional lines. One possible effect is to limit the capacity of both special- and general-purpose governments to enact effective policies. It also has the potential of shielding their actions from the public (Killian 2009).

\section{Conclusion}

Returning to the significance and purpose of the literature review, the focus of which is to highlight the salient theoretical frameworks that define the co-production of public sector organizational innovation within a special district. While the work of earlier theorists like Zaltman, Duncan, and Holbek (1973) and Van de Ven, Angle, and Poole (1989) provides a useful backdrop for understanding the complexity and interrelated nature of innovation it is the later theorists-in particular, Bason (2010) and Walker (2008) - that provide the best point of departure.

No generally agreed-upon and empirically tested theory of innovation in the public sector currently exists. Camison-Zornoza et al. (2004) assert that the literature 
offers no common integrating concept of organizational innovation. This is a result, they conclude, of differing and particularistic conceptualizations of the phenomenon. Indeed, the literature reflects a dominant tendency to employ qualitative methodologies, such as surveys or preexisting data sets, to infer theories that are fragmented and inconclusive in explaining the phenomenon (Camison-Zornoza et al. 2004).

This suggests the need for the development and operationalization of a flexible and adaptive theoretical framework for describing and explaining the causal mechanisms behind the co-production of innovation in the public sector. In addition to framing the research question, the research reviewed is section suggests a direction and propositions for future research. The critical next step is defining a theoretical construct for the coproduction of organizational innovation at the nexus of the organization, its leadership, and its customer or clients nested within a unique strategic environment. This is the subject of the next chapter. 


\section{CHAPTER 3: PUBLIC SECTOR ORGANIZATIONAL INNOVATION MODEL}

Innovation is an inherently social process. A unique blend of cognition and emotional intelligence is required for organizational innovation in the public sector (West and Farr 1990). A positivist approach to the philosophy of the social sciences, therefore, provides a useful framework for inquiries into this phenomenon. The social sciences provides the necessary tools to approach the critical task of conceptualizing the research design necessary to investigate this phenomenon. In the context of this dissertation, the research design provides the proposed path or procedural plan for an inquiry into this social phenomenon (Kumar 2011). It involves defining the bounds of this examination. This includes the methodical approach to data collection and analysis. This chapter defines the framework employed to explore this phenomenon.

Successful co-production of innovation is dependent on leadership, the participation of select segments of the leader's organization, and customers or clients. They all interact within a larger strategic environment which itself is the result of the prevailing political economy. This environment serves to mediate individual behavior though institutional mechanisms. Institutions provide the common language and shared experiences necessary for the individual collaboration required to co-produce organizational innovation. Individuals co-produce innovation in an effort to address common social challenges within a strategic environment conditioned by political and economic factors. Individuals, furthermore, have the ability to shape the strategic environment through various institutional mechanisms. 
In the model this dissertation puts at risk, innovation is the dependent variable. It manifests in the presences of particular antecedents. Certain independent variables serve to operationalize these antecedents. The presence of innovation is the result of causal mechanisms that occur because of a complex social dynamic. Antecedents associated with leadership, the organization, and the organization's customers or clients serve to activate the pathway to innovation. The result is the opening of the space necessary for innovation to occur. The socio-political environment in which these institutions operate serves to either advance or hinder the formation of the necessary pathway to innovation.

\section{Introduction}

This chapter presents the theoretical framework that describes organizational innovation in the public sector. Explaining this framework involves the clear articulation of the definitions and concepts used to describe this phenomenon. The theoretical framework represents a deductive-inductive typological approach to theory creation (King, Keohane and Verba 1994). The literature presented in the previous chapter provides the foundation and lays the groundwork for this theoretical framework. This this model seeks to explain how antecedents interact to produce a path that results in public sector innovation (George and Bennett 2005).

Antecedents serve to open the pathway to co-produced public sector innovation.

They are the causal mechanisms that operate to open the innovation space in public sector organizations. Antecedents are capable of operationalization through independent variables that serve as indicators of the antecedents. The independent variables reflect indicators. They are, therefore, empirically observable in a public sector organization 
when that organization innovates. There are, in other words, specific organizational antecedents that are present when innovation occurs. These antecedents are the causal mechanisms through which the co-production of public sector innovation occurs.

The more explicit the theoretical concepts that are employed, the more valid the description of organizational innovation in the public sector. This chapter, therefore, opens - after this brief introduction - with a section that defines the terms used in the creation and operationalization of the model. The third section presents a conceptualization of the model. Following that, this dissertation advances several associational statements that reflect some of the conceptual relationships existing between the components of the model. Associational statements are expressions regarding reality — they state a relationship without implying cause (Reynolds 1971).

The section that follows further refines the empirical orientation employed to describe and explain this phenomenon. This section articulates the methodological approach employed to operationalize the model. This dissertation proposes to validate the model by comparing measures of expected and actual organizational innovation in the public sector. If there are high levels of expected organizational innovation, and the model is valid, this will lead to the empirical observation of high levels of actual organizational innovation. Conversely, if there are low levels of expected organizational innovation and the model is valid, empirical observation will reveal low levels of actual organizational innovation.

Since public sector innovation is a social phenomenon, it is inherently difficult observe the causal mechanisms that lead to its creation. It is possible, nevertheless, to measure the quantity and quality of antecedents that the model suggests are present when 
organizational innovation exists. This occurs by employing variables. These variables serve as the basis for constructing two aggregate or summary measures that represent the phenomenon of public sector innovation. They are the public sector innovation organizational index (PSIOI) and the organizational antecedents of public sector innovation (OAPSI) rating. The former serves as a proxy measure of expected innovation and the latter represents the actual value of innovation subject to empirical observation.

This dissertation rests on the premise that it is possible to identify the expected level of innovation in public sector organizations through the public sector innovation organizational index (PSIOI). The PSIOI represents an integrative framework for measuring expected innovation in the public sector. The PSIOI serves to answer the question of whether it is possible to expect to observe the presence of innovation in a particular public sector organization. Each case studied received a single aggregate PSIOI score based on the evaluation of secondary source data.

An innovative organization is one that is resilient, accessible, efficient, and quality-driven. These four attributes or factors attach to the antecedents and are capable of empirical observation. They manifest in the variables that serve as measures of the antecedents. The higher the level of these four factors, the greater the degree to which these attributes manifest in an antecedent. This will lead to the empirical observation of increasing levels of organizational innovation. The converse is also true in which low levels of resilience, quality, accessibility, and efficiency indicate an expectation of low levels of actual organizational innovation.

This chapter also introduces the organizational antecedents of public sector innovation (OAPSI) rating. The OAPSI rating represents the actual measure of public 
sector innovation. It serves to link the empirical observation of the variables with the antecedents to operationalize the model. The empirical observations that resulted in the ratings occurred in the field through the individual interview process. The coding of the responses to the interview questions allowed for the assignment of empirical measures to the variables. Each interview question linked to the measurement of a variable and supported the creation of the OAPSI summary rating.

This research agenda involves determining and then validating the quantity and quality of the variables that indicate the presence of the antecedents that the model suggests provide the pathway to public sector innovation. The aggregate summary of these variables serves as a proxy measure for the independent variable of organizational innovation. It is the intent of this dissertation to suggest a cause-and-effect relationship such that antecedents create innovation. In other words, the antecedents open the pathway to public sector innovation. It is possible, furthermore, to describe and explain this causal pathway.

The eight section of this chapter outlines some of the ethical considerations faced in conducting this dissertation. It includes a statement of disclosure. The intent of this section is to demonstrate transparency as well as the quality and integrity of this research agenda. Other ethical considerations touched upon include insuring informed consent, assuring voluntary participation, and avoiding harm to participants. This section demonstrates that the research process is independent and impartial. In doing so, it respected the confidentiality and anonymity of the research respondents. This chapter ends with a brief concluding section. 


\section{Definition of Terms}

The previous chapter illustrated the challenges inherent in developing an empirical model of innovation. It remains elusive for theorists and practitioners alike. This is largely the case because innovation is a social phenomenon. As a social phenomenon, public sector innovation is difficult to quantify in sufficient specificity to measure in concrete terms (Little 1991). One of the results of this is that no generally accepted causal model of public sector innovation exists. Likewise, no generally accepted definition of public sector innovation exists. This study overcomes this gap by rigorously quantifying and measuring the variables that serve to operationalize the model of public sector innovation presented in this dissertation.

The lack of a common frame of reference makes dialog around public sector innovation challenging (Camison-Zornoza et al. 2004). Defining what is, and is not, innovation is a necessary first step in understanding the phenomenon. So is defining terms related to the co-production of public sector innovation. The working definitions proposed in this section are for the purpose of this study. The intent of this section is to provide definitions that avoid ambiguity and achieve clarity. The working definitions may vary from those of contemporary theorists but they have as their basis the work of earlier researchers.

The lack of generally agreed upon definitions of the concepts surrounding innovation exist in both the private and public sector. A quick survey of the definitions that various authors have proposed for innovation is illustrative. These examples represent some the more widely presented definitions of innovation: 
- "The intentional introduction and application within a job, work team, or organization of ideas, processes, products, or procedures, which are new to that job, work team, or organization and which are designed to benefit the job, the work team, or the organization" (West and Farr 1990).

- "Ideas which are new to the organization and result in value to the organization or its customers or clients. Innovation, therefore, is broadly defined and is manifest in products, processes, services, or in work organizations" (Weerawardena 2003, 20).

- "An intentional and proactive process that involves the generation and practical adoption and spread of new and creative ideas, which aim to produce a qualitative change in a specific context" (Sorensen and Torfing 2011, 849).

- "Any idea, practice, or material artifact perceived to be new by the relevant unit of adoption” (Zaltman, Duncan, and Holbek 1973, 10).

- "The tendency of the organization to develop new or improved products/services and its success in bring those products/services to market" (Gumusluoglu and Ilsev 2009, 266).

- "Generation and implementation of a management practice, process, structure or technique that is new to the state of the art and is intended to further organizational goals" (Birkinshaw, Gary and Mol 2008, 829).

- "A collective effort to produce and deliver public value that is authorized or sponsored by federal, state, provincial, or local government" (Hartley, Sorensen and Torfing 2013, 822).

One salient reason behind the lack of agreement on a common definition is that individuals conflate the various levels of innovation. The result is differing units of analysis. Differing units of analysis are a concern because they affect the analysis process (Dubin 1969). This is the case, in large measure, since the underlying assumptions that guide the analysis are dependent on the underlying assumptions tied to the unit of analysis employed.

It is often the case that assumptions vary among differing analytical traditions that seek to understand social phenomenon (Little 1991). Therefore, for example, an 
analytical approach to a particular unit of analysis may assume the aggregation of individual preferences while another may not. This can, and often does, result in differing conclusions from similar observations or data sets. According to this line of reasoning, it is possible for innovation - that occurs at the individual, team, group, organizational, institutional, or societal levels - to have differing antecedents depending on the individual or institutional context in which innovation occurs.

For this study, the definition of public sector innovation is systemic behavioral change that results from practices imbedded in institutions that both outperform previous practices and results in the common good. It represents organizational change that the preponderance of individuals — with knowledge of the organization — perceives as new. This definition has its genesis in the canonical works of Zaltman, Duncan, and Holbek (1973). In this regard, the artifacts of innovation manifest in the actions of individuals. Institutions, however, matter.

Institutions serve to shape individual actions. Individual actions, moreover, serve to structure institutions. This dynamic interplay results in universal acceptance of a new way of doing something. Simply put, innovation is a "change that outperforms previous practice" (Napier 2012, 32). In this setting, public sector innovation focuses on common goods and services. These good and services are capable of being shared among multiple customers and clients. It is possible for the scope of common interests, however, to be quite parochial in nature.

This definition of innovation departs from the perspective of Zaltman, Duncan, and Holbek (1973) by suggesting that innovation is inherently normative. The distinguishing feature of public sector innovation is that it orients on community values. 
From this prospective, society's beliefs and norms matter. The question arises; moreover, as to whose values matter and what social norms dominant. A defining characteristic of the public sector is that political institutions serve to arbitrate society's values (Morgan et al. 2013). Private sector innovation, conversely, stems from the desire to achieve individual interests through market mechanisms. In this context, the market serves to arbitrate norms and values through the structuring of economic relationships (Morgan et al. 2013).

Public sector innovation is dependent on the process of co-production. Ostrom (1996) defines co-production as "the process through which inputs used to provide a good or service is contributed by individuals who are not in the same organization" (1073). In this sense, the focus is on the input rather than the process of transforming inputs into outputs. Boyle and Harris (2009) further refine this definition by suggesting that co-production reflects the "means for delivering public services in an equal and reciprocal relationship between professionals, people using services, their families and their neighbors" (11).

This dissertation adopts Bovaird's (2007) definition of co-production as the provision of goods or services through long-term routinized relationships between professionalized service providers and service users or other members of the community where all parties make substantial resource contributions. This definition is consistent with Ostrom's (1990) conception of co-production. The model identifies service users as the customers and clients of the organization. Other members of the community could include citizens of the port district. 


\section{Conceptualization}

Concepts represent the distinct building blocks of theory (Reynolds 1971). They provided the symbolic meaning necessary to define the model in sufficient detail for operationalization. Operationalization refers to the process of reducing relatively nebulous theoretical concepts, like innovation, into indicators of the concept (Gibbs 1972). These indicators are then subject to empirical observation and measurement. In this way, it is possible to develop knowledge of a complex social phenomenon. Table 2 contains the definitions of the key terms presented in this chapter and used throughout the dissertation.

\section{Table 2. Definition of Key Terms}

\begin{tabular}{|c|c|}
\hline Term & Definition \\
\hline $\begin{array}{l}\text { Public Sector } \\
\text { Innovation }\end{array}$ & $\begin{array}{l}\text { Systemic behavioral change which results from practices imbedded in } \\
\text { institutions that both outperform previous practices and results in the } \\
\text { common good (various) }\end{array}$ \\
\hline Co-Production & $\begin{array}{l}\text { Provision of goods or services through long-term routinized relationships } \\
\text { between professionalized service providers and service users or other } \\
\text { members of the community where all parties make substantial resource } \\
\text { contributions (Bovaird 2007) }\end{array}$ \\
\hline Leadership & $\begin{array}{l}\text { Institutionally embedded and situationally appropriate practice of creating } \\
\text { relationships for the purposeful achievement of the public good (Shove, } \\
\text { Pantzar and Watson 2012) }\end{array}$ \\
\hline Organization & $\begin{array}{l}\text { Manifestation of the near-decomposability means of securing the benefits } \\
\text { of coordination while holding down the costs by an appropriate division of } \\
\text { labor among sub-units (Simon 2000) }\end{array}$ \\
\hline $\begin{array}{l}\text { Customers } \\
\text { and/or Clients }\end{array}$ & $\begin{array}{l}\text { Individuals in relationships for the purposeful achievement of the public } \\
\text { good (various) }\end{array}$ \\
\hline $\begin{array}{l}\text { Strategic } \\
\text { Environment }\end{array}$ & $\begin{array}{l}\text { Culturally-cognitive patterns and normative codes of thought and behavior } \\
\text { which exhibit rule-like regularity on individuals and institutions (Scott 2003) }\end{array}$ \\
\hline
\end{tabular}


Reynolds (1971) suggests that theories represent stories that individuals tell each other about the nature of reality. They are, by definition, abstract and result from an inductive process informed by intuition. Theory construction, therefore, reflects theoretical construction at the abstract level using pragmatic generalizations and intuition (Dubin 1969). Whether inductive or intuitive, the objective of this process is to understand the phenomenon under study in order to improve the human condition (Gibbs 1972). The ultimate test of any idea, however, is its utility in the face of reality.

This section defines the concepts that populate the model in such a manner as to ensure consistency throughout this dissertation. The purpose of this section is to explore in depth the conceptual structure of the theoretical model this dissertation puts at risk. What follows is an exploration of each conceptual component or element of the model. The purpose is to provide a firm foundation from which to operationalize the model in the subsequent chapter. Describing the concepts is also necessary to determine the location of boundaries between the elements of the model. It also serves to define the system state of the model. This was useful in developing the empirical indicators used to measure the values of the independent variables (Dubin 1969).

This section reviews the individual elements of the model and suggests how they interact with other elements or components in the strategic environment. The latter is the milieu with which the individuals and institutions involved in the co-production of public sector innovation must contend. The interactions result in, or create, the following domains:

1. Leader-client co-production

2. Client-organization co-production 


\section{Organizational-leader co-production}

\section{Innovation co-production}

The research literature outlined in the previous chapter suggests antecedents associated with each of these domains. These antecedents create the pathway to the co-production of innovation.

The general trend in the literature is to conceive of leadership and innovation as separate subjects only tangentially linked through theory and practice. The conceptual model this dissertation puts at risk fuses them into an integrated whole. Public sector leadership is defined as the institutionally embedded and situationally appropriate practice of creating relationships for the purposeful achievement of the public good (Shove, Pantzar and Watson 2012). In this context, leadership is dependent on emotional intelligence. This reflects the ability to manage oneself and one's relationship with others (Goleman, Boyatzis and McKee 2002).

As outlined in the previous chapter transformational leadership is a style of leadership characterized by the identification of needed changes, the creation of a vision necessary to guide the organization from inspiration to implementation, and the ability to form the necessary partnerships to bring the vision to fruition. The leadership necessary for innovation is present whenever there is an alignment between the practice of leadership and the ethical and effective institutional efforts to secure the public good (Nye 2008). In the context of this model, leadership is necessary, but not sufficient, to foster the co-production of public sector innovation.

The organization is an artifact of the dominant values that existed at the time of its creation (Morgan 2006). Organizations reflect the institutionalization of norms that 
emerged from these dominant values. These norms, and their resulting values, serve to shape the goals and objectives of the organizations by both constraining and restraining behavior. Organizations exist to coordinate goal-directed behavior among likeminded individuals in an economical manner (Mintzberg 1983). Leaders do so by attempting to exercising control over individuals through rewards or coercion.

An organization is a manifestation of the near-decomposability means of securing the benefits of coordination while holding down the costs by an appropriate division of labor among sub-units (Simon 2000). The ability to exercise discretion in coordinating and carrying out assigned divisions of labor is the essence of an organization (Mintzberg 1983). Within the context of this model, organizations are necessary, but not sufficient, to foster the co-production of public sector innovation.

Organizational structure and culture either support or impede organizational innovation (Morgan 2006). Organizational structure defines a social construct or hierarchy within an organization. Organizational structure identifies each job, its function, and where it reports within the organization. The structure of the organization conditions how individuals interact (Mintzberg 1983). It defines their individual roles and relationships. Organizational structure can be vertical, hierarchal, and rigid. This characterizes a traditional bureaucratic organization (Weber 1964). It can also be horizontal, flat, and flexible (Cooper 2003).

Organizational culture reflects the shared assumptions, values, and beliefs that govern how individuals behave in organizations. Organizational culture conditions the level of individual and organizational risk tolerance. The theoretic framework holds that the higher the level of risk tolerance, the greater the propensity for innovation. In other 
words, the culture of an organization modulates the risk tolerances of individuals. This is salient because the model assumes that the co-production of innovation involves engaging in responsible risk-taking. So, innovation occurs to the extent that an organization's culture values risk-taking behavior and tolerates responsible failure. The incentives that the organization employs to reward or punish behavior reveals the degree to which an organizations culture embraces risk. Within the context of this model, risk tolerance is necessary, but not sufficient, to foster the co-production of public sector innovation.

The individual or organization that benefits from the co-production of innovation is the customer or client of the public sector organization. The customers or clients of the public sector organization exist in relationship to the organization and share a common interest with other customers or clients in securing a public benefit that the organization provides. This is a particularly important aspect of special districts since their customers or clients often have a direct economic stake in the delivery of goods and services. Customers or clients are individuals in relationships for the purposeful achievement of the public good. In this regard, they participate in the co-production of innovation. They do so to maximize benefits while minimizing the shared costs.

Customers or clients control resources - either directly or indirectly — and they leverage this control to secure public goods and services. One of the critical resources they control is information. The model assumes that innovation in the public sector is stimulated by low levels of information asymmetry. Equal access to information has a democratizing effect that empowers individuals to achieve the public good. The model 
assumes that information asymmetry is a necessary, but not sufficient, condition to foster the co-production of public sector innovation.

Leaders and their customers and clients interact to co-produce public sector innovation though collaboration, cooperation, and interest alignment. Interest alignment is particularly important to securing the public good because it contributes to the achievement of the consensus necessary to define the public good. Adaptive interest alignment is, therefore, salient in achieving shared interests. In this context, one important factor is the existence of feedback loops (Sahni et al. 2013). Successful adaptive interest alignment suggests that the number and type of engagement opportunities are important for organizational innovation. Measuring successful adaptive interest alignment requires the ability to monitor both the process of innovation and the value of ongoing innovation efforts to key customers and clients of the organization.

An exchange of resources occurs through interactions between the organization and their customer or clients. This exchange is social in nature and reflects the prevailing political economy. In this regard, it reflects the dynamic interplay between society's economic, social, legal, and political institutions. The exchange, therefore, is not purely economic in nature. Further complicating this exchange is the fact that the object of this transaction is the common public good. This gives rise to a collective action problem (Olson 1971).

The result of this exchange of resources is the creation of value. It is through the exchange of tangible or intangible resources that the creation of value occurs. Tangible resources, in this context, include public goods and services. In addition to physical assets, resources can be monetary. Intangible assets can comprise information or political 
support. Regardless of the nature of the resources, the attributes of quality, cost, and leadtime combine to provide value. It is possible to characterize the interactions between customers or clients as based on individual transactions or systemic relationships. The theory and practice of co-production suggests that the presence of systemic relationships is necessary, but not sufficient, for innovation to occur.

The strategic environment in which leaders, organizations, and customers or clients embed is complex, uncertain, and multifaceted. It is a reflection of the prevailing political economy and signifies a complex tapestry of constraints and restraints that exist at multiple levels. The term political economy refers to the way societies organize the means of production as it relates to goods and services (King 1948). Thus, the distribution and arrangement of resources is critical to public sector innovation, as is the degree of interdependency and independence that organizations can exercise.

The strategic environment is those culturally cognitive patterns and normative codes of thought and behavior that exhibit rule-like regularity on individuals and institutions (Scott 2003). The strategic environment conditions the behavior of leaders, organizations, and customer or clients. Leaders, organizations, and customers or clients can also influence the strategic environment (Scott 2003). Institutional structures that embed within a unique strategic environment serve to moderate individual and institutional behavior around innovation.

Through the process of operationalization, it is possible to comprehend public sector innovation. This is the case even though the phenomenon is not directly measurable. The empirical validation of the model is separate and distinct from the formulation of the model (Dubin 1969). Validating the model requires the 
operationalization of the model. Operationalization requires the clarification of the concept of co-produced public sector innovation in sufficient detail to distinguish and describe it in terms of empirical observations. Operationalization involves describing what is, and is not, a manifestation of the phenomenon (Dubin 1969). In this regard, it is possible to discern the phenomenon of organizational innovation in the public sector.

It is possible to infer the existence of public sector innovation through observable effects. Dubin (1969) asserts that, "the empirical indicator of a unit of a theoretical model is an operation performed by an observer with some kind of observing instrument... [that] produces a value on the unit" (186). In this study, this occurs through participant observation. Participant observation involves the narrative recording of individual experiences and recollections related to public sector innovation. The case study approach provides the mechanism for accomplishing this task (Yin 1994). This involves making empirical observations under natural conditions in the field. In this dissertation, the observations and interviews occur concurrently.

\section{Associational statements}

Associational statements are expressions regarding reality. They are conceptual reflections of theory. Associational statements may reflect a relationship without implying a particular cause (Reynolds 1971). In the context of this dissertation, associational statements reflect the claim that certain antecedents are present during the co-production of innovation within the public sector. These antecedents create the pathway to co-produced public sector innovation. In other words, they describe a causal relationship. Associational statements are a powerful tool for identifying, describing, and 
empirically observing indicators of a phenomenon. Once observed, these indicator variables are susceptible to empirical measurement.

Reynolds (1971) suggests that formal relational statements only include two concepts that may vary. This is because it is only possible to conduct hypothesis testing, whether quantitative or qualitative, on two variables. This is not the case in this dissertation. This dissertation is testing just one relationship: the relationship between the theoretical model and the observed data. This approach includes one or more constant concepts within a formal associational statement to restrict the domain of the statement (Reynolds 1971).

Successful innovation is that which the organization routinizes and, furthermore, is manifest in discrete artifacts embedded in the organization. The presence of these artifacts is, consequently, evidence of innovation. Leaders can emerge from anywhere within the organization to champion innovation. They must be, however, effective change agents. Successful innovation requires the active participation of customers or clients. Select segments of the leader's organization, furthermore, must mobilize around the innovation for success to occur. This research project, therefore, advances the following proposition:

$P_{1}$ - Innovation is dependent on leadership capable of championing organizational change, the mobilization of select segments of the leader's organization, and the active participation of customers or clients. The presence of the antecedents the model predicts determines if this proposition holds. If this occurs, it implies that the proposed conceptual framework is operative. 
The question arises over whether innovation manifests in the absence of one or more of the antecedents the model suggests, or in cases where the weak form of the antecedents exist. This will call into question the utility of the antecedents in providing a causal pathway to innovation. Doubt or uncertainty over the model's validity or accuracy will occur at the point at which innovation transpires in the absence of the antecedents that the model suggests. The validity or accuracy of the model will also come into question in instances where the predicted antecedents are absent yet innovation is present. If either of these conditions exists then one of the formidable advantages of the case study method is "the heuristic identification of new variables and hypotheses" (George and Bennett 2005, 20).

If the predicted antecedents are absent, the model suggests that the empirical observation of instances of innovation are not possible. In other words, the organizational space necessary for innovation will not open. If the antecedents are present to a lesser degree, or in a weaker form, then the model suggests then it is possible to observe innovation to a lesser degree or perhaps not at all. In these instances, the model implies that organizational change may occur; nevertheless, it will not rise to the level of innovation. Change short of innovation could include organizational accommodation by one or more individuals that results from dynamic tensions. These dynamic tensions could result from attempts to resolve organizational dissonance.

There is also the danger of encountering a confounding antecedent. A confounding antecedent is analogous to a confounding variable (Reynolds 1971). In this regard, it represents an extraneous antecedent whose presence affects the model such that innovation occurs without regard to the presence or absence of the antecedent. In other 
words, the confounding variable is irrelevant in describing the causality of the co-

production of innovation in the public sector. This highlights the importance of identifying and controlling for possible confounding antecedents in the research approach. One possible safeguard against a confounding antecedent is a comprehensive literature review. Another safeguard is the employment of the case study method (Dubin 1969).

George and Bennett (2005) advise, "case-studies allow for conceptual refinements with a higher level of validity over smaller number of cases" (19). This is because the case study approach allows for the identification of indicators and the measurement of variables that best represents the phenomenon of interest. Table 3 summarizes the various conditions under which it is possible to observe the co-production of innovation in the public sector. This table provides insight in the various conditions that can emerge when attempting to provide an explanation for public sector innovation. This table also lays out the conditions under which the model is valid.

Table 3. Test of Implied Model Validity

\begin{tabular}{|l|l|l|}
\hline \multicolumn{1}{|c|}{$\begin{array}{c}\text { Empirical Observation of } \\
\text { Antecedents }\end{array}$} & \multicolumn{1}{c|}{$\begin{array}{c}\text { Empirical Observation of Innovation } \\
\text { Condition }\end{array}$} & \multicolumn{1}{c|}{$\begin{array}{c}\text { Implies Model } \\
\text { Validity }\end{array}$} \\
\hline \multirow{2}{*}{ Present } & Innovation Present & Yes \\
\cline { 2 - 3 } & Innovation Absent & No \\
\hline \multirow{2}{*}{ Not Present } & Innovation Present & Yes \\
\cline { 2 - 3 } & Innovation Absent & No \\
\hline \multirow{2}{*}{ Weak Form Present } & Innovation Present & Yes or No \\
\cline { 2 - 3 } & Innovation Absent & Yes or No \\
\hline Confounding Antecedents & Innovation or No Innovation & Yes or No \\
\hline
\end{tabular}

(Adapted: Yin 1994; Ragin 2014) 


\section{Empirical Orientation}

Theories and models in the social sciences seek to describe social reality (Dubin 1969). Reynolds (1971) defines theory such that it represents the notion that a set of abstract associational statements exist capable of describing those things that it is possible to know will happen with a high degree of certainty. This would imply that it is possible to validate a theory through empirically falsifiable statements about reality (Little 1991). From this perspective, it is feasible to derive sufficient empirical support using a positivist approach to the social sciences that meets the criteria of science as an epistemology.

This is the case since it is reasonable to posit the falsifiability of an abstract theoretical or conceptual statement through empirical observation (Almond and Genco 1977). It is possible to derive indicators of a concept through associational statements. These statements reflect the causal pathway that leads to innovation. It is possible, therefore, to either reject or accept a theory through empirical observation. Abstract concepts, moreover, are not temporally bound and, therefore, ensue independent of unique conditions or specific circumstances (Reynolds 1971).

The operationalization of the model follows a logical path from theory to concept to variable. The latter is capable of empirical observation and measurement (Dubin 1969). This represents a traditional path to operationalization adopted and adapted for use in this dissertation (Kumar 2001). The theoretical model reflects abstract associational statements regarding the reality of co-producing innovation in the public sector. The model exists in an axiomatic format that suggests relationships as a set of axioms that manifest as antecedents (Reynolds 1971). 
In terms of this dissertation, the theory under study depicts co-production of public sector innovation. This theory rests on concepts that describe the operation of the model. These concepts, in turn, reflect in the propositions advanced in this research project. They manifest as associational statements. The associational statements embed in the data collection instrument (see Appendix A). From the propositions, it is possible to derive variables that are capable of empirical observation and measurement. The case content analysis coding contains the details of this approach (see Appendix B). In this regard, the antecedents that the model suggests reflect in the indicator variables operative in the model.

The independent variables represent indicators of the antecedents that support the operationalization of an abstract theoretical statement. The case study approach allows for the empirical observation of cause and effect relationships through pathways that vary predictably (George and Bennett 2005). The antecedents reflect in the changing quantities of independent variables. Innovation, on the other hand, represents the dependent variable. Innovation, therefore, is dependent upon the antecedents. Given the complexity and social nature of innovation, it is not possible or desirable to control the independent variable.

Previous sections of this chapter presented a description and definition of each concept using a proposition that describes the dynamic nature of the model. The concept of public sector innovation is associated with an indicator of an antecedent that is capable of empirical observation and measurement (Reynolds 1971). Concepts provide the symbolic meaning necessary to define the model in sufficient detail such that it is possible to operationalize the model (Reynolds 1971). It is feasible from an 
epistemological perspective to measure concepts either quantitatively or qualitatively. Either type of measurement is equally valid and objective. It is possible to measure concepts at the nominal level—indicating no inherent ranking—or ordinal level— indicating ranking without a continuous ordering (Reynolds 1971).

This dissertation explores the experiences, meanings, and perceptions of individuals relative to co-produced innovation. It involved a scientific study of human society and social relationships. This dissertation is, therefore, located in the intellectual domain of the social sciences. This research project adopts the qualitative methodology of the case study approach to explore this phenomenon. The case study approach affords the investigator the "ability to contribute to the development of theories that can accommodate various forms of complex causality" (George and Bennett 2005, 5).

A good theory provides a parsimonious representation of reality. It serves to understand interactions and predict outcomes (Dubin 1969). The aim of theory is to provide a simplified explanation or depiction of a complex social phenomenon. George and Bennett (2005) recommend that the most useful theories are typological in nature. They reflect the intervening mechanisms or pathways through which the phenomenon manifests. Covering laws, on the other hand, reflect only correlations between the independent and dependent variables (Little 1991).

The qualitative empirical orientation of this dissertation infuses the data collection and fieldwork strategy (Singleton and Straits 2010). The former includes interviews, field observations, and document collection. Research methodologies create a link between the empirical evidence and a defined social phenomenon (Little 1991). This dissertation's methodological approach lends itself to describing variations that may support an 
alternate proposition. Research in the social sciences provides the insight necessary to build a theory and then validate that theory through empirical observation.

\section{Public Sector Innovation Organizational Index (PSIOI)}

This section, and the one that follows, defines the two indices that serve to measure the dependent variable of co-produced public sector innovation. The creation of a grounded theory of co-produced public sector innovation preceded the development of the indices. The indices serve to quantitatively frame the empirical observations. The indices serve to validate the theoretic framework this dissertation put at risk. The PSIOI represents the expected value of co-produced public sector innovation. This measurement paradigm reflects two differing perspectives on public administration.

Two contending movements emerged to deal with the countervailing social demands for less government, on the one hand, and more public goods and services on the other. One response to these conflicting social expectations in the United States was the New Public Management (NPM) movement (Morgan and Shinn 2014). NPM sought to impose market discipline on the process of public governance. Imposing a business ethos on the bureaucratic structure would regulate relationships and frame individual behaviors in a socially beneficial manner (Green 2014).

The focus of the NPM paradigm was on the effective and efficient delivery of public goods and services. The New Public Governance movement, on the other hand, emerged to address issues of community and social connection absent from NPM. This movement sought to ground the authority of networked governance in the underlying social values and legal principles of the prevailing political system (Morgan and Shinn 
2014). The intent was to weave a tight social fabric. This movement was a reaction to the impersonal transactional nature of the NPM approach.

Good public governance is both accountable to its citizens for accomplishing the common public good and capable of achieving the common public good (Manin, Przeworski and Stokes 1999). The former is what NPG seeks to achieve while the latter is the focus of NPM (Morgan and Shinn 2014). The model of public sector innovation this dissertation puts at risk bridges the NPM and NPG paradigms. It seeks to combine those elements of good governance that most effectively and efficiently achieves a positive and beneficial social outcome. The model of public sector innovation, furthermore, is normative in nature. It accepts the proposition that public governance is laden with conflicting values and interests. In this sense, institutions endure as artifacts of the dominant values that exist at their formation (Schein 2004).

The public sector innovation organizational index (PSIOI) balances the NPM's focus on the proper administration of government and embeds it in the NPG's emphasis on the normative creation of public goods and services (Morgan and Shinn 2014). The literature review on the measurement of innovation in chapter 2 suggests that some organizational factors can emerge to form components useful in measuring organizational innovation (EU 2013; APSC 2011; Reese 1996). Various researches, furthermore, hold that it is possible to conceptualize organization innovation in terms of attributional indicators subject to empirical observation (Ven, Angle, and Poole 1989; Adams, Bessant and Phelps 2006). This suggests that it is conceivable to assign values to various factors or conditions that contribution to the emergence of public sector innovation (Walker 2008). 
The model suggests organizational innovation expected to be present when the antecedents of innovation exhibit four attributes: resilience, quality, accessibility, and efficiency. Each of these attributes reflects the salient qualities that exist concurrent with organizational innovation in the public sector. These attributes bias an organization towards innovation. Each of these four attributes represent a quality or feature of the antecedent that is a characteristic of, or inherent in, organizations that engage in public sector innovation.

When these attributes attach to the various antecedents then institutions exhibit an institutional preference for engaging in innovation. This is not to suggest that they necessarily spark innovation or that a mechanistic cause-and-effect relationship exists. The model suggests instead that a salient bias for innovation exists concurrent with high levels of organizational innovation and the presence of these antecedents. In other words, the presence of the antecedents causes innovation while the attributes of the antecedents define their salient characteristics.

The attributes of resilience, quality, accessibility, and efficiency define and shape the measurable indicators of the antecedents that a researcher would expect to observe when organizations innovate. While the direct measurement of the concept of innovation is not possible, it is conceivable to rely on other means that are logically reflective of the concept (Kumar 2011). It is feasible to operationalize these antecedent attributes within the scope of these indicators. In this regard, it is possible to assign variables. This involves the creation of a categorical scale that is both qualitative in nature and capable of empirically measuring indicators of public sector innovation. It is, furthermore, 
possible to combine these variables and their values to create a composite index that serves as a measurement of expected organizational innovation in the public sector.

The public sector innovation organizational index (PSIOI) serves to operationalize, in measurable terms, a potentially subjective concept. This involves identifying indicators of the attributes of resilience, quality, accessibility, and efficiency that can display variance across organizations. The qualitative approach represents the best methodology for dealing with perceptions or beliefs regarding occurrences of innovation. In the context of the PSIOI, attributes of quality and efficiency are characteristic of NPM while resilience and accessibility are hallmarks of NPG. The conceptual foundations of the antecedent's links to each of these four attributes and each attribute individually serves as a complement to the others.

The first attribute, quality, serves to provide an understanding of what constitutes performance and the resulting common good that an organization produces. The concept of innovation links to the attribute of quality because both concepts, at least in the context of the public sector, are in large measure a reflection of both processes and outcomes. The ISO 8402-1986 standard defines quality as "the totality of features and characteristics of a product or service that bears its ability to satisfy stated or implied needs." In other words, quality is a measure that is a standard of something compared to something else.

Deming (1986) argues, "Innovation, the foundation of the future, cannot thrive unless top management has declared unshakable commitment to quality" (25). This is the case, he goes on to theorize, in large measure because quality cannot emerge from an environment characterized by "unstable chaos" (11). Bason (2010) suggests, moreover, 
that some degree of organizational stability is necessary for an organization to innovate. Quality is, in other words, in large measure the product of stability. This is an apparent contradiction since stability runs counter to the disruptive nature of innovation.

The second attribute, efficiency, is the ability to produce a good or service with a minimum amount of effort (Deming 1986). In other words, it is the capacity to produce a desired good or service with a minimum of expense. Cost can exist, moreover, directly or indirectly as hidden opportunity costs or waste. An efficient organization is one that possesses the discipline necessary to focus on innovation in the pursuit of institutional success. Deming (1986) asserts that efficiency is, in large measure, a result of quality since improvements in quality lead to efficiency improvements. The insight - that productivity improves with quality_is Deming's (1986) essential contribution. In other words, quality improvements are the result of practices that outperform previous practices.

Resiliency is the third attribute and allows the organization the capacity to innovate and achieve organizational success in a volatile, uncertain, complex, and ambiguous strategic environment (Walker 2010; Kantur and Iseri-Say 2012). Kantur and Iseri-Say (2012) suggest that organizational resilience is the "capability that helps the organization to evade successfully the continuous disturbances of volatile business environments and reach ultimate organizational aims effectively" (764). They postulate a framework for conceptualizing organizational resilience.

The four-category framework that Kantur and Iseri-Say (2012) propose identifies the sources of organizational resilience as a perceptual stance, contextual integrity, strategic capacity, and strategic acting. They argue that a perceptual stance suggests the 
mental orientation necessary for resilience, whereas contextual integrity integrates cognitive and behavioral modalities that emerge from the perceptual stance. Strategic capacity and acting, on the other hand, are action-oriented and incorporate factors that turn a perceptual stance into reality (Kantur and Iseri-Say 2012).

Accessibility, the fourth attribute, is essentially normative and serves as the catalyst for systemic behavioral change. In the historical parlance of the United States, accessibility is almost exclusively legal or regulatory in nature. Therefore, for example, it refers to the government's role in assuring goods and services are available for individuals with various levels of physical capabilities. This is a US-centric view that contrasts with the European approach to accessibility. The latter approach recommends a much broader concept that allows individuals to achieve specified goals within the context of social inclusion (Farrington and Farrington 2005).

Farrington and Farrington (2005) propose a conceptual framework in which "the greater social justice cannot be achieved without greater social inclusion, which requires that people have access to a range of activities... greater social inclusion requires greater accessibility" (2). Accessibility, therefore, institutionalizes the relationships necessary to create the common good. Accessibility refers to the ability to design products, devices, services, or environments that maximize collective social benefits through social inclusion (Farrington and Farrington 2005).

The measurement of the variable involved assessing the frequency, direction, significance, and robustness of the individual samples collected during the research phase of this study. This study design served to operationalize the concept of public sector innovation by converting the concept of public sector innovation into various indicator 
variables and then assigning measurements to each (Kumar 2011). This allowed for the empirical measurement of public sector innovation. The intent of the PSIOI is to answer the question: "Is it possible to expect to empirically observe innovation in a given public sector organization?" This dissertation uses existing secondary data sets that are publicly available as the sample source for these variables. The use of these secondary sources of data had the benefit of providing a potentially neutral, independently derived data set.

Measuring the PSIOI variables involved assessing the resilience, quality, accessibility, and efficiency of each variable and then combining them to create a composite index. This measurement scale relied on descriptive statements contained in Table 4 to identify the variables in such a manner that it is possible to assign values (Kumar 2011). Regarding the unit of measurement, Kumar (2011) states that "qualitative variables are similar to categorical variables as both use either nominal or ordinal measurement scales" (72). From this perspective, it is possible to identify instances of the variable and assign values to the variables that reflected the frequency, direction, significance, and robustness of the attributional indicator. The unit of measure employed was a composite scale that used ordinal (or ranking) measurements to indicate high, medium, or low values.

The approach to the unit of measurement used in this dissertation suggests that the attributes that make up the PSIOI have a relationship to one another such that it is possible to identify meaningful variation. If this is not the case, it will not return a consequential result. The composite is characteristic of a nominal scale with regard to the antecedent's attributes. Each antecedent attribute, moreover, has a relationship such that it is possible to assign values to each variable in an ascending or descending order. The 
composite scale reflects this possibility. It is important to note that how a variable is measured drives the type of analysis the researcher can perform (Kumar 2011). This has the potential of limiting the utility of this index.

\section{Table 4. Definition of PSIOI Terms}

\begin{tabular}{|l|l|}
\hline \multicolumn{1}{|c|}{ Term } & \multicolumn{1}{c|}{ Definition } \\
\hline Quality & $\begin{array}{l}\text { Totality of features and characteristics of a product or service that enables } \\
\text { its ability to satisfy stated or implied needs. }\end{array}$ \\
\hline Efficiency & $\begin{array}{l}\text { Capacity to produce a desired good or service with a minimum of expense. } \\
\text { Resiliency } \\
\text { disturbances of volatile business environments and reach ultimate } \\
\text { organizational aims effectively. }\end{array}$ \\
\hline Accessibility & $\begin{array}{l}\text { Ability to design products, devices, services, or environments that maximize } \\
\text { collective social benefits through social inclusion. }\end{array}$ \\
\hline
\end{tabular}

(Adapted: ISO 8402-1986; Deming 1986; Kantur and Iseri-Say 2012; Farrington and Farrington 2005)

Each port in Oregon and Washington operates a publicly accessible website on which they publish a wide variety of material on the port and its operations. In addition, several organizations on the state, regional, and hemispheric level collect, compile, and report data on public ports in Oregon and Washington. This is typically secondary to their function of protecting and advancing the common interests of their member ports. These public sector organizations include the American Association of Port Authorities (AAPA), the Northwest Marine Terminal Association (NWMTA), the Washington Public Ports Association (WPPA), and the Oregon Public Ports Association (OPPA). 
The AAPA represents over 130 public port authorities throughout in the US, Canada, the Caribbean, and Latin America. Their member ports vary in size, structure, and mission (AAPA 2012). Through these four associations, Oregon and Washington ports seek a collective voice in advocating and protecting their common interests. As an example, the AAPA seeks to be "the industry's voice on governmental policies that strengthen and expand opportunities for member ports" (AAPA 2012, 3). There is a risk in using AAPA data since there is a possibility it could have a bias skewed in support of its membership.

\section{Organizational Antecedents of Public Sector Innovation (OAPSI)}

This section outlines the framework for operationalizing the model of the strategic dimensions of public sector organizational innovation. The implied theoretical basis for this model serves to frame the conditions under which the model is operative (Singleton and Straits 2010). The empirical observation of independent variables that serve as indicators of the antecedents associated with the leader, the public sector organization, and their customers or clients is possible. The presence of the antecedents leads to the existence of public sector innovation. What follows in this section is a discussion of the model's interrelationships.

The OAPSI reflects the actual level of organizational innovation. This section presents the methodological approach to measuring the ten variables that indicate the presence of the six antecedents that create the pathway to the co-production of public sector innovation. This model represents a middle-range typological theory. Typological theories, according to George and Bennett (2005), "identify recurring conjunctions of 
mechanism and provide hypotheses on the pathways through which they produce results" (8). The intent of the OAPSI is to answer the question of how innovative a given public sector organization is. It does so in an empirical manner.

In this model the leaders, the organization, and their customers or clients are the constituent parts. Each of these parts exists in relationship to the other parts and embed within a distinct strategic environment. Antecedents are the emergent artifacts of the dynamic interaction between the constituent parts. This theoretical framework takes into consideration internal and external factors - and especially the influence of the strategic environment. In other words, the model seeks to frame the circumstances under which innovation emerges in the public sector.

The model seeks to explain the pathway through which the antecedents coproduce public sector innovation. Units of analysis exist as the major entities that this dissertation seeks to analyze and understand (Heclo 2008). The unit of analysis for this study is the observed practice of individuals embedded in institutions engaged in relationships involving the co-production of innovation in the public sector (Heclo 2008). In other words, the unit of analysis for this dissertation is the individual engaged in organizationally appropriate relationships that result in the co-production of innovation in the public sector.

Antecedents manifest in the shared space between the leader, organization, and customers or clients. These shared spaces represent the domains of the model. Domains are the shared space that opens between two or more constituents in which co-production emerges. Differing independent variables emerge to shape and define the relationship in each of the domains. Therefore, for example, organizational structure, culture, and a 
shared sense of collective organizational consciousness modulate the relationship between the leader and their organization in the domain of organizational-leader coproduction. Each domain has a predominant focus. Therefore, for instance, an input and output focus characterizes the domain of organization-leader co-production.

The first of the three constituent components considered is leadership. This model posits that leaders emerge from differing hierarchal levels or functional areas within the organization to champion innovation. This does not mean that senior leadership is unaware or not involved in innovation; however, it does recognize that the capacity to mobilize the organization and engage customers or clients exists throughout the organization. Leaders animate other actors towards the creation and sustainment of organizational innovation. This can take the form of creating interpersonal alliances, networks, and informal organizations that operate alongside or within existing institutional structures for the purpose of innovation.

Leaders rely on diffuse sources of power to champion innovation. This, for example, can include referent or expert power capable of social influence (French and Raven 1959). It also includes the ability to address the psychological component of organizational innovation. Leaders also adopt a comprehensive, relationship-based perspective that takes into account the complex political, social, and economic environment within which they operate (Bass 1985). In this regard, leaders exercise leadership on a situational basis.

The second constituent component under consideration is the public sector organization itself. The model asserts that organizational boundaries adjust to include the subgroups necessary for organizational innovation. Organic to the organization are the 
resources necessary for innovation and the pathway to acquiring these resources. This includes the human, financial, technological, and decision-making means necessary to execute innovation. This is not to suggest that the organization's resources are the only means available. Indeed, public sector organizations often face constraints and restraints on the available resources. This often requires organizations to explore alternative sources of resources.

An important component of the ability to innovate is the organization's capacity to acquire the necessary resources. This occurs, in large measure, through the ability to bargain across or within organizational boundaries. The organization this model characterizes is risk tolerant such that it is both willing and able to experiment. Risk tolerance exists to the extent that the institutional culture celebrates failure alongside success. Core values and principles supportive of co-production are operative under conditions of innovation. This serves to condition the courses of action available.

The final constituent component is the organization's customers or clients. The model theorizes that customers or clients serve to signal the existence of gaps between existing socio-economic conditions and the organization's governing capacity. Conditions of information asymmetry that favor the customer or client result in increased opportunities for the active involvement of customers and clients. Conditions of information asymmetry that favor the leader or the organization result in fewer opportunities for customers and clients to initiate or participate in innovation.

The relationship between the leader and customer or client is manifest in the domain of leader-client co-production. Figure 3 contains a depiction of this relationship. It is from the domain of leader-client co-production that it is possible to observe one of 
the antecedents - adaptive interest alignment. The model suggests that it is possible to measure adaptive interest alignment thorough two variables. The first is the robustness and intensity of feedback loops. The second is leading with a vision. Together these two independent variables serve to operationalize adaptive interest alignment.

\section{Figure 3. Leader-Client Co-Production Domain}

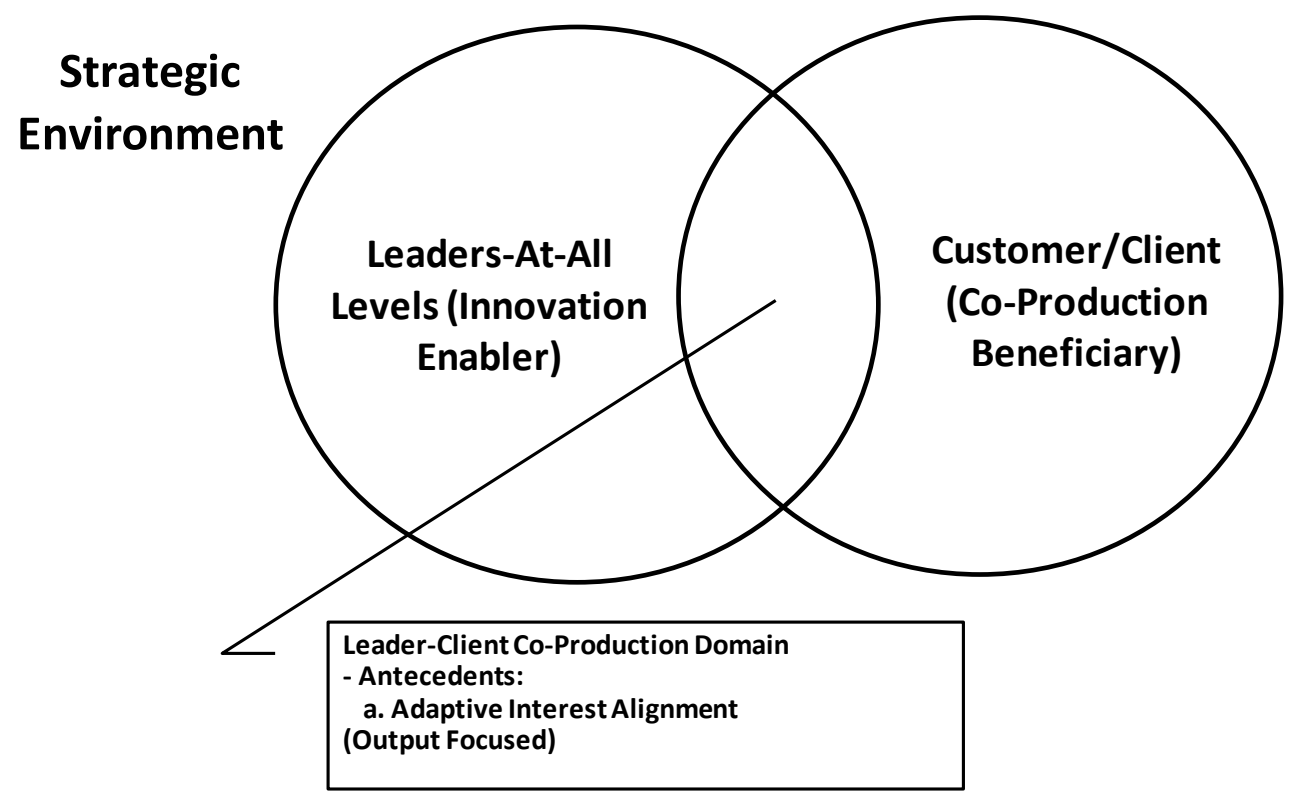

It is in the domain of client-organization co-production that the antecedents of client-based prioritization and co-production readiness manifest (see Figure 4). Clientbased prioritization and co-production readiness are the two antecedents operative in this domain. The model posits that there are two indicator variables tied, respectively, to each of these antecedents. It is possible to measure client-based prioritization through the existence of resource constraints and co-production gap awareness. It is feasible, 
furthermore, to measure co-production readiness though information symmetry and the ability to sunset products and processes.

\section{Figure 4. Client-Organization Co-Production Domain}

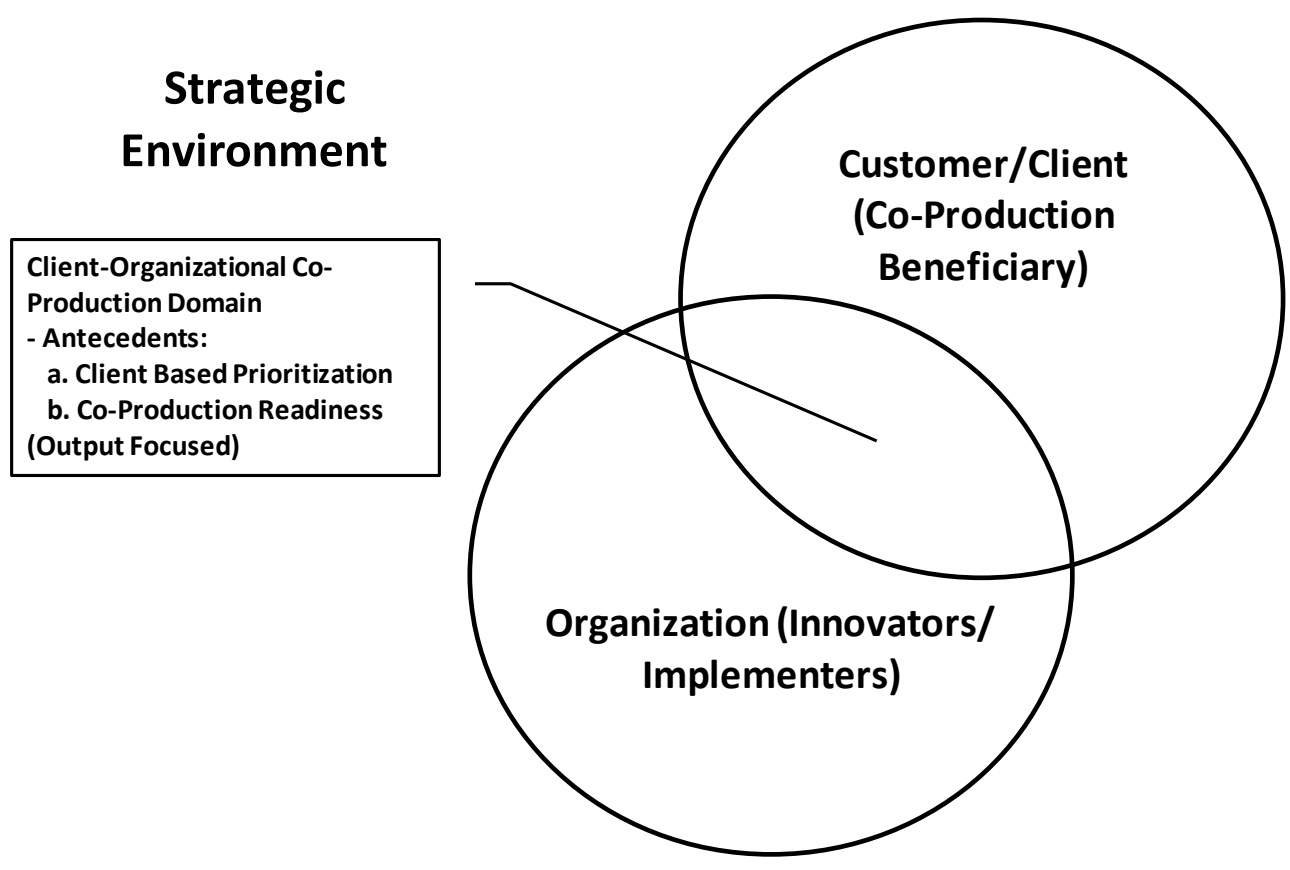

The model posits that the antecedents of organizational incentives, risk-taking preferences, and organizational structure and culture manifest in the domain of organizational-leader co-production (see Figure 5). Organizational structure is an artifact of organizational culture and reflects the values of an organization. It is possible to measure the antecedent of organizational culture and structure though the presence of transformational leadership and the ability to experiment. These two independent variables serve to operationalize organizational structure and culture. Incentives for improvement activity, conversely, serve as the measurable variable of the antecedent of 
organizational incentives. Individual risk-taking preferences serve as an independent variable of the antecedent of risk-taking preferences.

Figure 5. Organization-Leader Co-Production Domain

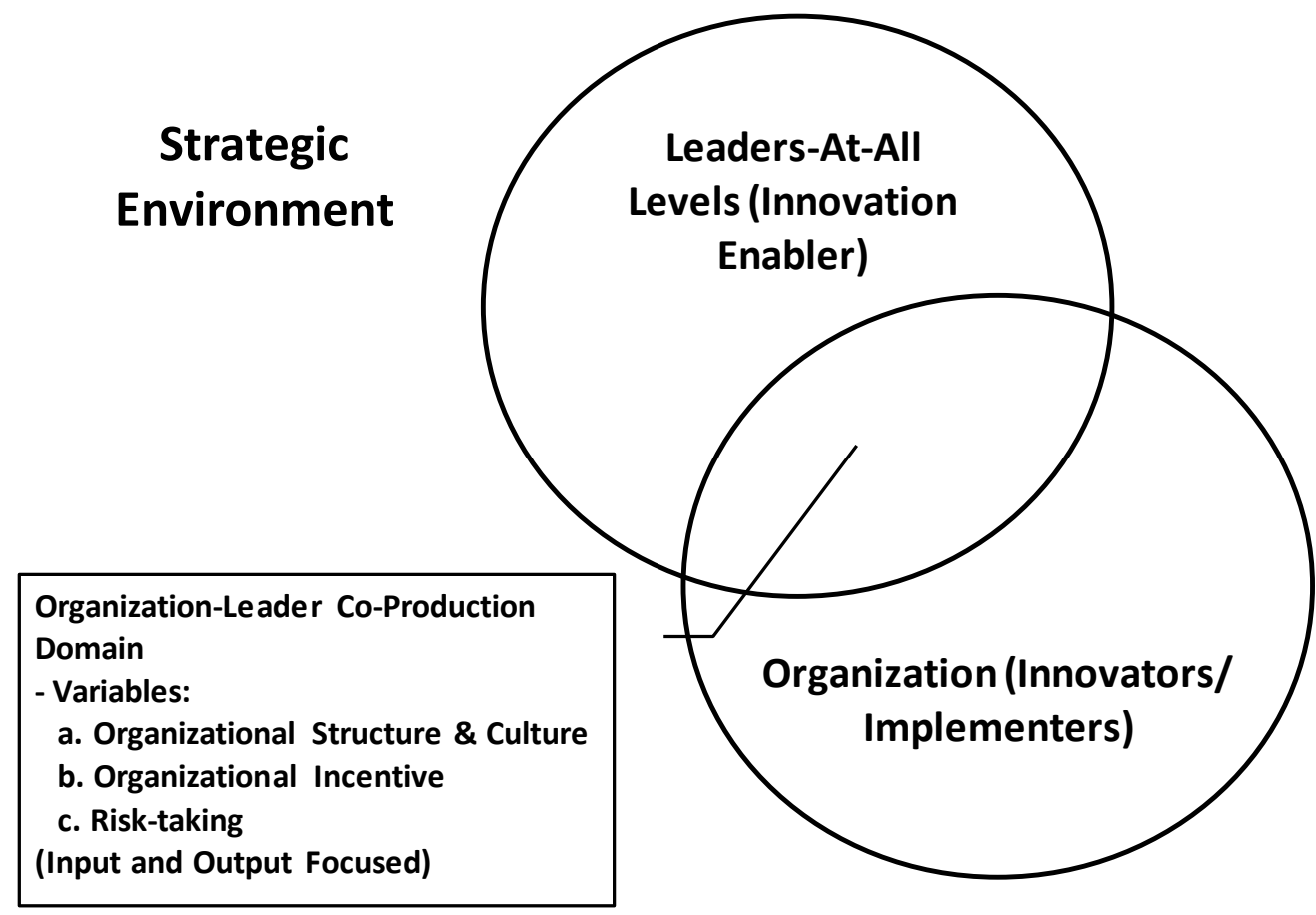

The model holds that the co-production of innovation occurs in the space that opens within the confluence of the various domains. It is possible to observe the independent variables that serve as indicators of the antecedents in each of these domains. The model posits that innovation is not possible without all three constituent parts acting in harmony. It occurs, furthermore, in the presences of the antecedents identified in this section. Each antecedent imbeds in the various domains the model postulates. Chapter 4 outlines in detail the process of operationalizing this dissertation's model. 
In the context of this model, the constituent components operate individually to create the synergy necessary to co-produce innovation. It takes a unity of effort borne of a unity of purpose to open the domain of innovation. It is important to note that the model suggests that antecedents operate through dyadic relationships in the leader-client, clientorganization and organization-leader co-production domains. These dyadic relationships are socially significant. Cumulatively, the antecedents operate to foster innovation through a distinct pathway.

Table 5 provides a summary of the domains, antecedents, and variables that animate the model. The ten independent variables serve as the basis for the PSIOI and the OAPSI rating. Each variable serves to operationalize one or more of the antecedents that creates a pathway for innovation to occur. Antecedents represent a concept the dissertation measured. In this regard, an antecedent may have multiple variables. Each variable, however, ties to the operationalization of only one antecedent. Each antecedent, moreover, is unique to a specific domain. The domains represent the core theoretical constraints of the model. Each of these independent variables serves as an image, perception, or concept that is capable of measurement (Kumar 2011). The PSIOI and OAPSI rating is an empirical measure composed of the individual variables.

The presence of feedback loops and leading with a vision are the two independent variables that operationalize the antecedent of adaptive interest alignment. Adaptive interest alignment resides within the leader-client co-production domain. Evidence of routinized communications regarding innovation processes and projects serves to measure the existence of feedback loops. Feedback loops serve to create and strengthen the pathway necessary for aligning interests. Evidence of the ability to articulate 
innovation in relation to an institutions vision or mission statement serves to measure leading with a vision. This is the case since interests adapt and align to organizational and individual interests.

Table 5. Organizational Antecedents of Public Sector Innovation (OAPSI)

\begin{tabular}{|c|c|c|c|c|c|}
\hline \multicolumn{2}{|r|}{ Domain } & \multicolumn{2}{|r|}{ Antecedent } & \multicolumn{2}{|r|}{ Independent Variables } \\
\hline \multirow[t]{2}{*}{1} & \multirow{2}{*}{$\begin{array}{l}\text { Leader-Client } \\
\text { Co-Production }\end{array}$} & \multirow[t]{2}{*}{1.1} & \multirow{2}{*}{$\begin{array}{l}\text { Adaptive Interest } \\
\text { Alignment }\end{array}$} & 1.1 .1 & Existence of feedback loops \\
\hline & & & & 1.1 .2 & Lead with a vision \\
\hline \multirow[t]{4}{*}{2} & \multirow{4}{*}{$\begin{array}{l}\text { Client- } \\
\text { Organization } \\
\text { Co-Production }\end{array}$} & \multirow[t]{2}{*}{2.1} & \multirow{2}{*}{$\begin{array}{l}\text { Client-Based } \\
\text { Prioritization }\end{array}$} & 2.1 .1 & Existence of resource constraints \\
\hline & & & & 2.1 .2 & Co-production gap awareness \\
\hline & & \multirow[t]{2}{*}{2.2} & \multirow{2}{*}{$\begin{array}{l}\text { Co-Production } \\
\text { Readiness }\end{array}$} & 2.2 .1 & Sunset through co-production \\
\hline & & & & 2.2 .2 & Information symmetry \\
\hline \multirow[t]{4}{*}{3} & \multirow{4}{*}{$\begin{array}{l}\text { Organization- } \\
\text { Leader Co- } \\
\text { Production }\end{array}$} & \multirow[t]{2}{*}{3.1} & \multirow{2}{*}{$\begin{array}{l}\text { Organizational } \\
\text { Structure/Culture }\end{array}$} & 3.1 .1 & Transformational leadership \\
\hline & & & & 3.1 .2 & Ability to experiment \\
\hline & & 3.2 & $\begin{array}{l}\text { Organizational } \\
\text { Incentives }\end{array}$ & 3.2 .1 & $\begin{array}{l}\text { Incentives for improvement } \\
\text { activity }\end{array}$ \\
\hline & & 3.3 & $\begin{array}{l}\text { Risk-Taking } \\
\text { Preferences }\end{array}$ & 3.3 .1 & Individual risk-taking preference \\
\hline
\end{tabular}

The existence of resource constraints and co-production gap awareness are the two independent variables that serve to operationalize the antecedent of client-based prioritization. The antecedent of client-based prioritization resides within the clientorganization co-production domain. Evidence of specific demands of customers or clients for innovation that reflects in organizational budget priorities serve to measure the existence of resource constraints. The existence of resource constraints drives prioritization. Evidence that organizations actively co-produced operational budgets that embraced needed public service innovations with customers or clients serves to measure the existence of a co-production awareness gap. While the existence of resource 
constraints drives prioritization, it is an awareness of gaps that creates a focus on customers or clients.

The ability to sunset through co-production and information symmetry are two independent variables that operationalize the concept of co-production readiness. To sunset something is to remove it from existence. The antecedent of co-production readiness resides within the client-organization co-production domain. Evidence of systematically examining and phasing out current processes, technology, or human resources in relation to innovation serves to measure the ability to sunset through coproduction.

The model holds that the manifestation of the phenomenon of public sector innovation is dependent on the readiness of the organization and the customer and client of the organization to engage in co-production. Phasing something out is one reflection of that readiness because it clears the temporal space for something new. Routinized information sharing and resource dedication to information sharing serves to measure the degree of information symmetry. Readiness to co-produce is also dependent on a shared base of information and knowledge.

The model posits that the existence of transformational leadership and the ability to experiment serve to operationalize the antecedent of organizational structure and culture. The antecedent of organizational structure and culture resides within the organizational-leader co-production domain. Evidence of three or more characteristics of transformational leadership serves to measure the existence of transformational leadership. Evidence of three or more systemic, time-phased experiments tied to one or more innovation initiative serves to measure the ability to experiment. 
The leader, the values they bring to the organization, and the way they animate the organization to achieve the leader's vision shapes the structure and culture of an organization according to the model. Two aspects of this are the nature of leadership and its manifestation. In this regard, the measure of the former relates to its transformation aspects and the later to an observable event like experimentation. The process of transformational leadership opens the pathway to innovation. Experimentation, on the other hand, is a manifestation of the route to innovation.

Incentives for improvement activities operationalize the antecedent of organizational incentives. The antecedent of organizational incentives resides within the organizational-leader co-production domain. The model suggests that evidence of both intrinsic and extrinsic incentives linked to supporting or participating in the coproduction of innovation serves as a measure of organizational incentives. Leaders control the provision of incentives that, in turn, drive organizational behavior. Control of these resources and the manner in which the leader distributes them can either impede or reinforce individual behavior towards innovation.

Risk-taking preferences operationalize the antecedent of risk-taking preferences. The antecedent of risk-taking preferences resides within the organizational-leader coproduction domain. Evidence of an expressed willingness to take risks when the reward is equal to or greater than the risk involved serves to measure individual risk-taking preferences. Innovation is an inherently risky activity. This is the case since failure to innovate successfully results in the loss of resources. The level of risk tolerance, therefore, signals an appetite for innovation. 
Appendix A contains the data collection instrument or interview guide used for this study. It includes the list of questions designed to elicit a measure of the independent variables during an in-depth subject interview. While it appears in a question and answer format, its main intent is to remind the researcher of the areas to cover during the interview. Appendix B contains the codebook used for this study. The codebook lists the numerical values assigned to the answers obtained from subjects during the field research phase of this study.

\section{Ethical Considerations and Statement of Disclosure}

Research ethics involves the moral principles that govern the planning, conduct, and reporting of the research results. The ethical practice of social science research requires this dissertation to confirm with a generally agreed-upon set of social science principles. It also requires the adherence to an academic code of professional conduct. Adhering to strict research standards the participants receive prudent protections from the real and perceived risks involved in this study. The first step in providing protection involves identifying the potential stakeholders.

Ascertaining the identity of the stakeholders makes it possible to identify, through careful analysis, the risks and benefits of this research agenda. The primary stakeholders in this research effort are the participants themselves, the researcher, and the sponsoring institution. In this dissertation, the author is the principle researcher. The sponsoring institution is the Portland State University (PSU). PSU is a public, nonprofit, coeducational research university located in Portland, Oregon. Because PSU is a research 
university, it has an institutional review process for social sciences research involving human subjects.

Secondary stakeholders include the academic theorists and public sector practitioners interested in public sector innovation. Academic theorists and public sector practitioners are the primary consumers of this research. The public - the ultimate benefactor of the study - represents an important secondary stakeholder as well. For these stakeholders, the researcher owes a duty to insure this dissertation represents a valid and beneficial contribution to the body of research on public sector innovation since the practice of public sector innovation is an inherently normative undertaking. If the study is undertaken based on a haphazard or ill-conceived methodology or conceptual understanding it may waste scarce public resources and fail to secure a common public good. In the worst-case scenario, it could end up doing harm.

There are a number of institutional safeguards built into this study, which insure that the primary and secondary stakeholders receive adequate protection. One of these safeguards is the Portland State University Institutional Review Board (IRB). The purpose of the board is to regulate research activities involving human subjects. The objective is to minimize the risks inherent in social sciences research. Copies of this dissertation's IRB application and supporting documentation are on file with Portland State University.

The IRB process provides an independent assessment of the methods, risks, and benefits present in the human subjects research this dissertation undertook. The purpose of the IRB process is to protect the rights of the participants. The intent of the process is to insure the informed consent of the study participants. The research, nonetheless, 
presents a very low risk to the participants. The dissertation committee also provides another layer of institutional protection. Committee oversight insured the research was in accordance with generally accepted codes of conduct and commonly agreed-upon scientific research principles.

These institutional safeguards provide important protections to stakeholders. Ultimately, however, the responsibility falls to the researcher to insure the use of proper research protocols. In this regard, there is a duty to conduct research that affords the highest possible level of protection to the research participant consistence with the risks involved. There are several possible sources of harm. As such, there is a requirement to define or identify the specific hazard or source of harm to a research subject. The primary source of harm comes from the disclosure of sensitive or proprietary information that could provide an embarrassment to the participant or their respective organization. Revealing sensitive information could damage the reputation or professional relationships of the participants. Additionally, the disclosure of confidential business information could result in economic harm to the organizations involved.

There is also a danger that participants could perceive their identity or identifying information did not, or could not, receive the proper level of protection. In this circumstance, it is likely they may not participate in the research or may provide incorrect or misleading information. Maintaining confidentially was, therefore, of utmost importance. Also of immediate importance was developing a high level of trust between participants and the researcher. This was vital for the collection of valid information. The researcher received informed consent from each participant. This occurred prior to the interview. This insured each subject was aware of the goals, objectives, risks, and 
benefits of research. Participation was purely voluntary and the subject could stop the interview at any time.

Ethical issues that involve the researcher can manifest in many ways. It is important to note that the researcher has a duty to avoid harming primary, secondary, and tertiary stakeholders. Harm can result from the existence of conscious or unconscious bias. There is also a danger of incorrect reporting or the inappropriate use of information that could indirectly harm the stakeholders by providing misleading, hurtful, or contradictory information. It is necessary to guard against these dangers by carefully considering the manner and method of study in close consultation with the dissertation committee members. The same goes for guarding against the use of inappropriate research methodologies. As a member of the community under study, there is a possibility that the researcher could inappropriately gain personally or professionally from information gathered during the course of this study.

There also exist potential ethical issues regarding the sponsoring organization-in this case Portland State University. This could involve the imposition of inappropriate research restrictions or issues involving the use of the information gathered from the participants. Research restrictions have the potential for limiting the scope of the inquiry in such a manner as to prevent a proper inquiry or a distortion of the results. It is also possible to use information from social sciences research in inappropriate ways. Portland State University is a public institution of higher education. Advancing a research agenda that benefits the public at large presents a minimal risk to the university.

It is important to disclose that I am a member of the study population so that it is possible to properly assess and evaluate my research. I have interacted closely with 
individuals employed at public marine ports in the states of Oregon and Washington. I have served as a private sector manager of several private marine-related enterprises that operate at public marine ports, including the Pacific Northwest. I have also been an employee at a public marine port in the Pacific Northwest. As expected, there were no ethical issues encountered during the study.

Further, I have been an active participant in the Northwest Marine Terminal Association (NWMTA) where I served in an official capacity representing a public port. I have also served on, and have chaired, various NWMTA committees and subcommittees involved in topics ranging from port security to NWMTA administration. I have retired from the US Navy after 30 years of service. During the course of my US Navy career, I was involved with national defense programs and naval operations across the globe. This includes extensive experience with foreign and domestic governmental organizations and dealing with matters related to public and private marine port operations.

I have, therefore, deep and extensive personal and professional connections with the subjects and organizations that are the subject of this study. The intent of this statement of disclosure is to ensure transparency regarding my personal and professional connections. While intimate familiarity with the subjects and organizations under study provides easy access, it may also result in inherent bias from experience or exposure to the population. By taking the steps outlined in this section, I was able to ensure this dissertation adhered to the highest ethical standards.

In order to minimize any potential bias that may result, this study excludes from the potential population of ports any organization in which I have a direct past, present, or known future employment or other economic interest. This minimized any potential 
conflicts of interest between a commitment to academic integrity and any inappropriate personal or professional gains that may result from this effort. By doing this, I was able to ensure the achievement of honesty and rigor in the research and any academic or professional dissemination of the information obtained in this study.

\section{Conclusion}

Sorensen and Torfing (2012) suggest that "the dearth of studies focusing on... innovation in the public sector is not least regrettable in light of the increasing demand for public sector innovation" (844). This dissertation closes this gap and adds to our knowledge of the theory and practice of innovation. This dissertation's unique contribution is to create and empirically validate a conceptual framework that explains and predicts the co-production of public sector innovation. This chapter lays out the model for the co-production of public sector innovation based on the research literature presented in the previous chapter. The next chapter outlines the process of validating this theory.

The empirical validation of the model is the topic of the remaining chapters of this dissertation. The validation of the model occurred through a study of three cases. In each of these cases, the phenomenon of public sector innovation was manifest. This dissertation relies on within-case analysis and cross-case comparisons to further our understanding of the pathway through which the antecedents that the model suggests creates the space for the co-production of public sector innovation. The empirical setting for the study of this phenomenon is special districts, specifically the Pacific Northwest ports of Oregon and Washington. 
The OAPSI establishes the expected level of innovation present in an organization. The PSIOI fixes the actual level of innovation in an organization. In other words, the OAPSI rating serves to validate the PSIOI. The PSIOI and OAPSI indices and their associated independent variables serve to measure the dependent variable of coproduced public sector innovation. The index and ratings measure innovation by assigning values to variables that indicate the presence of antecedents that open the pathway to innovation in the public sector. 


\section{CHAPTER 4: OPERATIONALIZING THE MODEL}

The previous chapter outlined the theoretical framework for understanding and predicting the co-production of innovation in the public sector. This chapter will outline how this dissertation puts the co-production model at risk. It does so through the lens of empirical observation. This theoretical framework posits that certain antecedents create the pathway through which public sector innovation occurs. It is possible to observe and predict the dependent variable of innovation through an understanding of the interaction of the antecedents. The model suggests several independent variables exist that indicate the presence of the antecedents.

The previous chapter contained a conceptualization of the model, outlined the research design, defined the terms used in this dissertation, and presented the empirical orientation used to validate the model. In the prior chapter, there was an identification of some of the problems and limitations of this study as well. Chapter 3 also introduced the public sector innovation organizational index (PSIOI) and the organizational antecedents of public sector innovation (OAPSI) rating. What follows is a discussion of the manner in which the PSIOI and the OAPSI rating serve to operationalize the model.

\section{Introduction}

This chapter opens with a presentation of the strategy, approach, and design employed in conducting the empirical research necessary to operationalize and validate a theory that explains and predicts the co-production of public sector innovation. While the previous chapter introduced the index, rating, and associated variables that measure the 
social phenomenon of co-produced public sector innovation, the sections that follow detail in some specificity the tools, techniques, and procedures employed in this study to operationalize the model. The previous chapter and what follows in this chapter lay out how the case study approach supported the operationalization of the model.

Dubin (1969) asserts that operationalization serves to articulate the model in terms of processes that illustrate its function. In doing so, it provides a measure of validity. In this regard, a model is valid if it mirrors the phenomenon under study in such a manner that it is possible to predict the co-production of innovation in a public sector organization in the physical world (Dubin 1969). This chapter contains an outline of the data collection and the instrumentation method employed in this dissertation. This study employs the method and logic of a focused, structured comparison (George and Bennett 2005). This involved using the case study approach to understand and predict the emergence of the co-production of innovation in the public sector.

This chapter contains a detailed description of the method used to create the public sector innovation organizational index (PSIOI) and the organizational antecedents of public sector innovation (OAPSI) rating. These indices served to operationalize the model. Chapter 5 presents three cases that used the approach outlined in this chapter to operationalize the model. This chapter wraps up with a section that outlines the case study orientation and layout followed in chapter 5. The strength of this study lies in "examining cases within the specified domain of the theory to see if their processes and outcomes are as the theory predicts" (George and Bennett 2005, 22). The cases are the result of empirical observation. The concluding chapter of this dissertation contains an analysis of the cases. 


\section{Research strategy}

The case study design strategy reflects a combination of naturalistic inquiry with emergent design flexibility (Patton 2002). The latter reflects a desire to avoid the existence of any predetermined constraints on the inquiry. The former intimates an open approach to adapting the inquiry as a deeper understanding of the social phenomenon emerges. This design strategy allows for the pursuit of new paths of discovery as they emerge (Singleton and Straits 2010). It should also prevent bias through the entry of predetermined constraints on findings or rigid design features that would prevent a robust exploration of the co-production of public sector innovation. The goal is to pursue a nonmanipulative and non-controlling study of a contemporary phenomenon.

Innovation represents a social phenomenon that is not amenable to understanding through a strictly quantitative approach. The lack of a generally agreed-upon theory of public sector innovation means that a combination of approaches is necessary depending on the object of the research study. In this context, it is misleading to categorize innovation as a strict function of express numerical values. Theory building requires a thick description of the social phenomenon to pave the path to a robust description, understanding, and causal explanation (Little 1991).

In this regard, "case-studies remain much stronger at assessing whether and how a variable mattered to the outcome than at assessing how much it mattered" (George and Bennett 2005, 25). Public sector innovation requires a thick explanation, which is only possible through a qualitative approach (Little 1991). What is at stake in selecting the lens through which innovation is viewed is the inevitably effect it has on understanding 
this phenomenon. Whatever approach is adopted, moreover, brings with it inherent strengths and weaknesses.

The philosophy of social sciences provides the most appropriate intellectual framework to improving the understanding of the co-production of public sector innovation. This is the case, furthermore, whether the researcher is seeking to observe, describe, explain, or predict occurrences of innovation in the public sector. Figure 6 places this dissertation within the philosophy of the social sciences. The philosophy of social sciences provides the context in which it is possible to define, describe, and explain the concept of innovation in the public sector.

Figure 6 reflects the ontology and epistemology of the research design. The epistemology of the case study approach-discussed in detail in the next section of this chapter describes how it is possible to know the existence of this phenomenon (Yin 1994). The ontology of the organization provides the basis of this dissertation's social reality. The contested nature of this phenomena emerged from the literature in chapter 2 . The positivist philosophy of the social sciences research design paradigm found within this dissertation provides a way to reconcile the contested nature of this phenomenon (Little 1991).

\section{Research Approach}

Three separate and distinct approaches to research dominant the social sciences. They are the experimental, survey, and case methodologies (Yin 1994; Thomas 2011). Ragin (1987) suggests that the former two approaches are quantitative in nature due to their orientation on variables. Conversely, Ragin (1987) asserts that the case approach is 
qualitative in nature. Survey research is a technique for the collection of data pertaining to a universe or population of interest (Freeman 1977). The researcher then subjects the data to statistical analysis that results in an associational description of the phenomenon (Little 1991). Experimental research is a technique for determining causality through the control of variables (Kumar 2011). The utility of this approach is limited to situations in which it is possible and desirable to manipulate social phenomenon.

\section{Figure 6. Philosophy of the Social Sciences Research Design Paradigm}

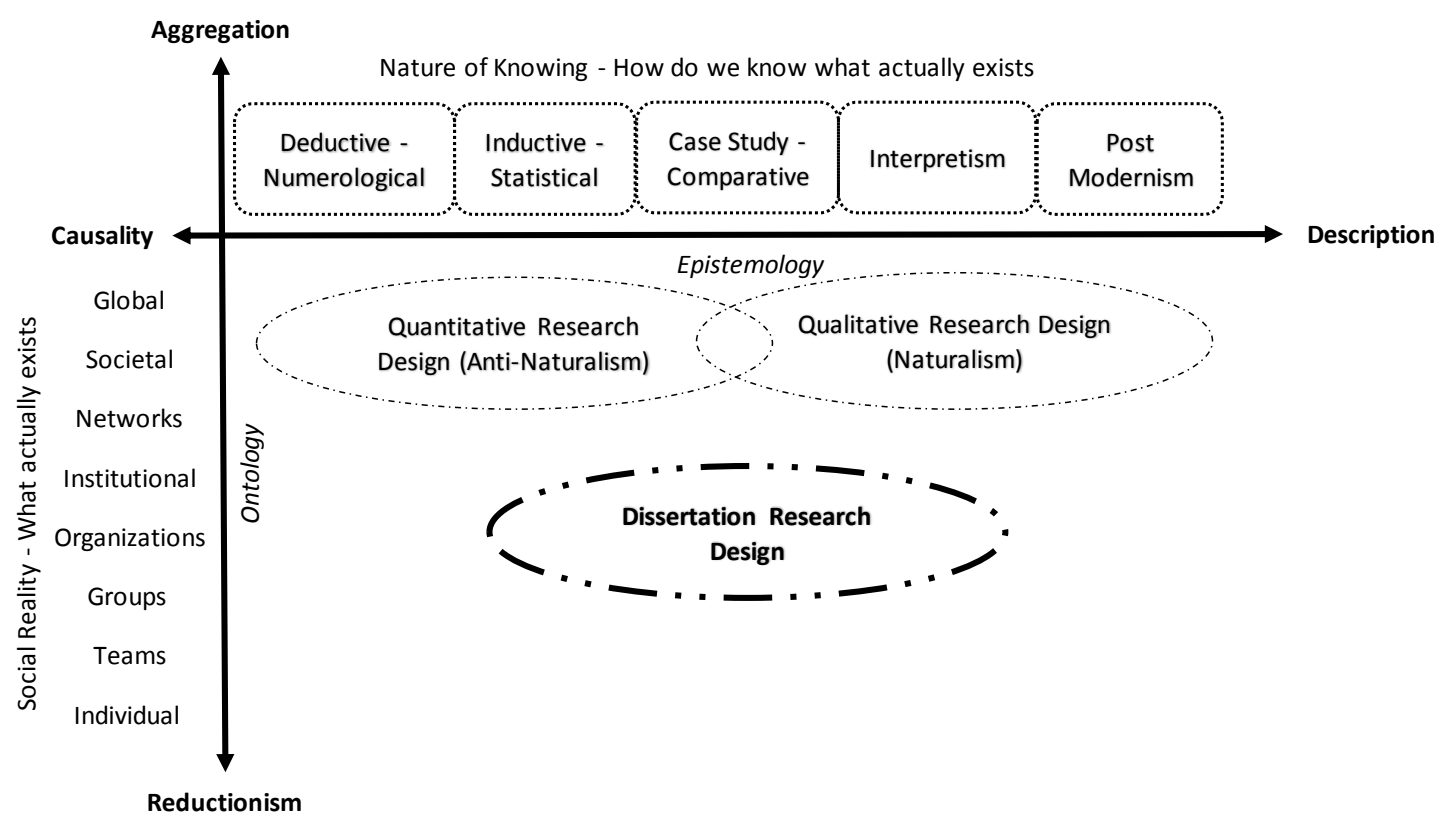

(Adapted from: Little 1991; Sen 1977; King, Keohane and Verba 1994)

To these three approaches, Yin (1994) would add the archival analysis and historical approaches. This dissertation omits these two from consideration since they are temporal in nature. This is case because this dissertation's study of innovation is contemporary in nature. It is necessary to undertake a contemporaneous study of this 
phenomenon if the implications, findings, and conclusions are to have practical utility today. In other words, understanding public sector innovation in the present rather than the distant past is the goal of this dissertation.

The experimental, survey, and case approaches each have advantages and disadvantages. The selection of a particular approach, therefore, is dependent on several factors related to the nature of the inquiry. Yin (1994) suggests that the type of research question, the degree of control the investigator is able to exercise, and the temporal focus conditions the selection of an approach. Thomas (2011) expands the list to include probability factors, the aim of the research, the nature of the information collected, and the complexity of the phenomenon. Ragin (1987) posits that the context of the inquiry as it relates to the complexity, diversity, and uniqueness of the phenomenon are factors as well. Pennings et al. (1999) would add to this the nature of the phenomenon (social or natural) that is the focus of the inquiry.

The experimental, survey, and case approaches to social sciences research are scientific in the sense that each encompasses a set of methods and procedures capable of formulating, testing, and modifying hypotheses through systematic observation and empirical measurement (Thomas 2011). Ragin (1987) posits that it is possible to use a case approach to approximate experimental rigor "by identifying comparable instances of a phenomenon of interest and then analyzing the theoretically important similarities and differences among them" (31). Yin (1994) asserts that it is feasible to validate a theory by logically linking data from two or more cases to the theoretical proposition. He goes on to suggest that replication of results is possible if data from subsequent cases links to the theoretical proposition. 
Yin (1994) argues that it is possible to consider multiple cases as one would consider multiple experiments. It is replication, and not sampling logic, that proves the theory. This is possible though comparative case analysis. In other words, theoretical replication is possible through the contrasting of results between cases. Multiple cases, therefore, equate to multiple experiments. Comparison does not require control. King, Keohane, and Verba (1994) argue that the conditions for a strict, controlled comparison are difficult to meet in the study of social phenomenon. This is the case because social phenomenon is the province of individuals acting as reflective agents (George and Bennett 2005).

Mill's (1843) method of agreement provides a useful paradigm for demonstrating the scientific nature of the case study approach. In this regard, Mill (1843) suggests that when a researcher observes two or more instances of a phenomenon, and they each have one specific circumstance in common, then that circumstance is the cause of the phenomenon. In essence, the case study becomes a search for patterns of invariance (Ragin 1987). Mill reasons, however, that the only way to establish a causal relationship is though experimentation.

George and Bennett (2005) criticize Mill's approach as being unrealistically strict, asserting that the "investigator cannot be sure that all the possible relevant independent variables have been identified or that the study has included a sufficient variety of cases of the phenomenon" (156). Another problem with Mill's method of agreement is the existence of multiple causation. In this regard, George and Bennett (2005) argue that differing causal patterns leading to similar outcomes, which they refer to as "equifinality," has profound implications for efforts to develop empirical theory. 
Ragin (1987) argues that when they are present, multiple causes are equivalent at the conceptual level. In other words, their presence constitutes a single invariant cause. The case approach, therefore, accommodates "multiple conjectural causation" in instances of causal complexity (Ragin 1987, 167). The case study is a focused investigation meant to describe and explain the dynamic sequence of human actions and complex social relationships inherent in a phenomenon. This approach, therefore, is ideally suited for the investigation of combinations of conditions that this model recommends. Causality is the result of the combination the antecedents. Each antecedent must be present in order for the co-production of public sector innovation to occur, as opposed to some combination of any two of them or something different altogether.

The approach a social science researcher adopts to study a phenomenon is dependent on the nature of the research. Ragin (1987) suggests that the case approach is best when dealing with invariant patterns common to a relatively small set of cases. This approach stimulates a rich dialogue between ideas and the case as a whole when the outcome of the phenomenon is more or less consistent across relevant observations. The cases are, in other words, contextually similar. The experimental and survey approaches are best for dealing for probabilistic relationships within a collection of variables (George and Bennett 2005). These approaches are particularly well suited to the study of phenomena with a small set of common variables.

Table 6 presents a summary comparison of the three dominant research approaches in the social sciences discussed in this chapter. Perhaps the greatest distinction between the case approach and the experimental or survey approach is the number and complexity of the components or mechanisms of the phenomenon under 
study. The case study approach seeks to develop a "three-dimensional picture" necessary to derive analytical insights (Thomas 2011,4 ). This approach is ideal for dealing with complex social phenomenon like the co-production of public sector innovation.

Table 6. Comparisons of Methodological Research Approaches

\begin{tabular}{|c|c|c|c|}
\hline \multirow[t]{2}{*}{ Features } & \multicolumn{3}{|c|}{ Research Approach } \\
\hline & Case & Experimentation & Survey \\
\hline Typology & Large; Complex & Small; Simple & Small; Simple \\
\hline Sample Size & Small & Large & Large \\
\hline Probability & No & Yes & Yes \\
\hline Control & No & Yes & No \\
\hline Quantification & Low Priority & High Priority & High Priority \\
\hline Method & Multiple & Single & Single \\
\hline Goal & $\begin{array}{l}\text { Relationship/ Process } \\
\text { Understanding }\end{array}$ & Causation & Generalizability \\
\hline
\end{tabular}

(Adapted from: Yin 1994; Pennings et al. 1999; Thomas 2011)

The case study approach is fundamentally holistic with an emphasis on understanding the organic or functional relationships between the parts and the whole. The experimentation and survey approaches, on the other hand, are atomistic in nature. They seek understanding through the division of the phenomenon into separate and often disparate elements (Pennings et al. 1999). None of these three approaches is inherently superior. They are, rather, complementary in nature. Each of these approaches has intrinsic strengths and weaknesses in pursuing description, explanation, and causality in the social sciences. It is, therefore, up to the researcher to select the approach that most closely aligns with their research agenda.

The ability of investigators to manipulate variables is one of the defining characteristics of experimentation. Both the experimental and survey approaches seek a 
sample of sufficient size to make probabilistic inferences regarding the population or phenomenon under study (Yin 1994; Pennings et al. 1999; Thomas 2011). The case approach, on the other hand, represents a multifaceted idiographic study of a phenomenon such that the sample size is relatively small (Thomas 2011). Cases are, therefore, non-probabilistic in nature.

Ragin (1987) observes that in the case and survey approaches it is often not possible or even desirable to achieve control over behavioral variables. Yin (1994) suggests that case studies are the preferred strategy "when the investigator has little control over events, and when the focus is on a contemporary phenomenon within some real-life context" (1). Yin (1994) defines a case study as "an empirical inquiry that investigates a contemporary phenomenon within its real-life context, especially when the boundaries between phenomenon and context are not clearly evident” (13).

For both the experimentation and survey approaches, obtaining quantifiable data is a high priority. This is necessary to establish causation in the experimental approach and the generalizability to larger populations in the survey approach (Yin 1994). Pennings et al. (1999) suggests that in this regard both the experimentation and survey approaches aim for breath of coverage. The focus of the case approach, on the other hand, is on depth of understanding and not quantification. This is because the goal is to understand complex relationships and social processes. In other words, the case approach represents a focus — not a methodology or procedure (Thomas 2011).

Public sector innovation presents itself as a multifaceted social phenomenon that is not easily unraveled (Walker 2010). This makes it difficult to explain in an atomistic manner. This is the case because individual, organizational, social, and political 
phenomena all combine in complex ways. Ragin (1987) suggests that the case approach provides an avenue for dealing with this complexity. This is because cases "tend to be very sensitive to human agency and to social processes in general” (Ragin 1987, 70). The observation of which does not require control over the behavior.

It is not possible or desirable for the researcher to manipulate the relevant behaviors in certain instances. The inability to control social phenomenon is one of the defining characteristics of social science research (Little 1991). King, Koehane, and Verba (1994) assert that while individual and organizational behavior is observable, the mental processes that create it are opaque. This perspective reflects the underlying assumption that individual and organizational behavior is organic and not subject to mechanistic controls (Scott 2003).

Thomas (2011) posits that the quality of a case study is dependent on its conception, construction, and conduct. He further suggests that a valid and reliable case study design for social sciences research consists of specific elements that exist within the following framework: subject, purpose, approach, and process. Thomas (2011) suggests that by applying this classification framework or typology it is possible to map out the design of a case study appropriate to a particular research agenda. This is especially the case when the research subject is a social process.

Applying the Thomas (2011) framework to the study of public sector innovation is useful in articulating the utility of the case study approach to understanding this phenomenon. The first element of the Thomas (2011) typology is, naturally enough, the subject or categorization of the inquiry. As pointed out in chapter 2, the choice, or subject, of public sector innovation is of inherent interest. Case selection hinged on the 
presence of co-produced public sector innovation. In other words, the basis for the selection is the outcome. In this regard, the Thomas (2011) framework suggests that a case study approach is appropriate. The second element is the purpose. In this instance, the purpose of the case study approach is instrumental.

Instrumentality reflects one of the purposeful elements of the classification framework (Thomas 2011). In this regard, the case approach serves a particular purpose. The aim is to provide a theoretical model for public sector innovation. The purpose is also explanatory in the sense that it may yield explanations based on a depth of understanding. Its purpose is also causal in the sense that it reflects the reasons for the phenomenon (King, Keohane and Verba 1994). The ultimate utility of a theory, in the final analysis, is the degree to which the framework accurately models complex social phenomenon (Dubin 1969).

According to the Thomas (2011) classification framework, the approach represents the third element of a valid case study. George and Bennett (2005) note that the case study methods supports several different types of theory-building research objectives. These include theory testing and plausibility probes. The purpose of the former is to assess the validity and scope of a single or competing theory. Plausibility probes involve preliminary studies of a relatively untested theory or hypotheses to determine whether more intensive or in-depth testing is necessary.

Thomas (2011) defines theory as an explanatory model and building a theory as developing "a framework of ideas, a model, that somehow explains the subject" (112). The final element in the Thomas (2011) typology is process. Process represents the structure of the case study. It is, therefore, critical to the research design. This dissertation 
employs multiple comparisons where "the focus is unequivocally on the phenomenon of which the case is an example" (141). This represents the comparative or cross-case analysis approach (Pennings et al. 1999; Ragin 1987).

This dissertation's approach passes the Thomas (2011) framework test. It is, therefore, a valid approach to understanding public sector innovation. Pennings et al. (1999) suggests that the "main advantage of the comparative approach in political and social sciences is to "test' theories by controlling contextual variation" (39). Ragin (1987) emphasizes the case-based nature of comparative research in which the analytic approach is to link configurations of causally relevant conditions to outcomes. In this regard, the aim of the comparative approach is to explain social processes using a theoretical framework. Ragin (1987) suggests that the goal of comparative social sciences is to explain and interpret macrosocial variation.

\section{Research Design}

The challenge the social science researcher faces is to construct meaning from social situations. Thomas (2011) points out that such "situations cannot be fractured into variables" (121). In this regard, the holistic and interpretive nature of the case-oriented approach is well suited for validating the theoretical model. Comparative case analysis, furthermore, represents a theory-driven method for conducting empirically analytical research. In other words, it represents a scientifically based research design paradigm (Pennings et al. 1999). Figure 7 outlines the research design using the case study approach followed in this dissertation. 
Research design, according to Pennings et al. (1999), reflects the number and cases selected, indicators of the phenomenon, and the analysis mode. They further note that a single case represents the unit of observation. Inherent in each case, therefore, are indicators of the phenomenon. Ragin (1987) also makes a distinction between observational and explanatory units. The former reflects the unit used in data collection and analysis (the case) while the latter accounts for the results (the outcome the model or theory seeks to explain).

\section{Figure 7. Case Study Approach Research Design}

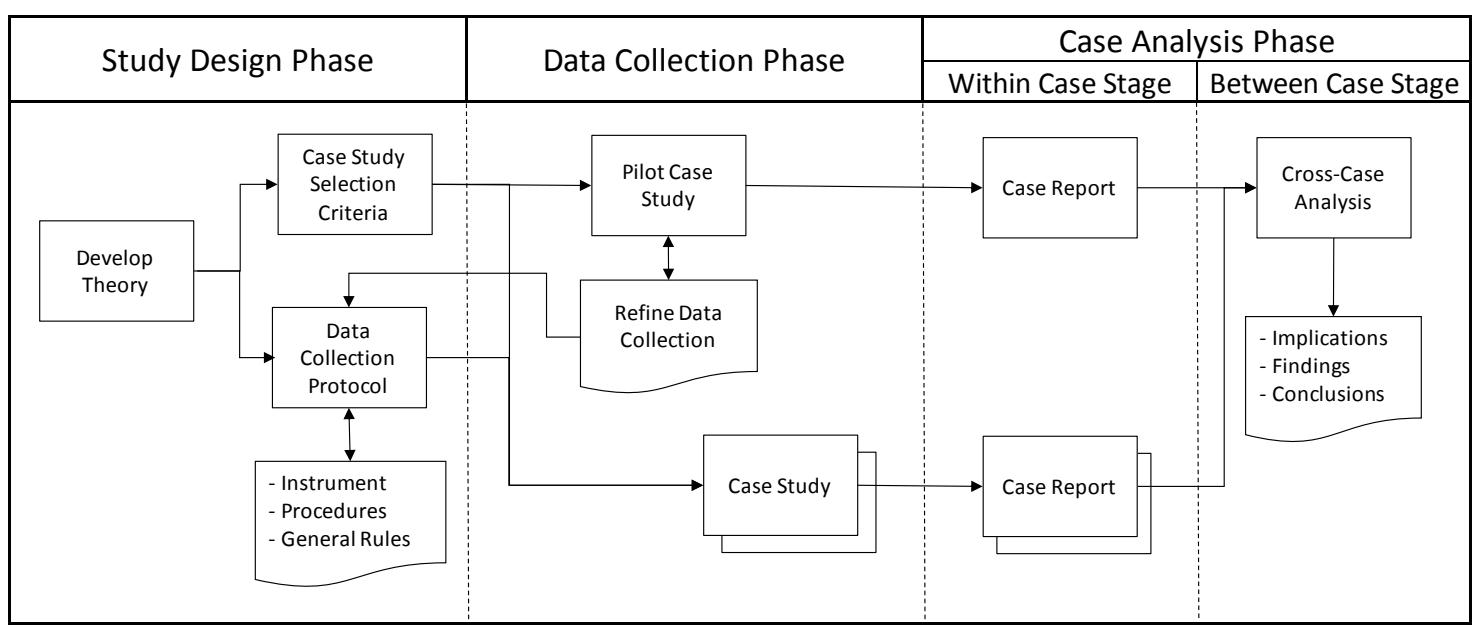

(Adapted: Ragin 1987; Yin 1994; Kumar 2011)

Pennings et al. (1999) suggest that indicators of the phenomenon vary. The indicators, thereby, serve as an inherent control in cross-case analysis since they should vary together. The operationalization of concepts occurs through units of variation and they are subject to quantification through units of measure. Pennings et al. (1999) assert that in the case approach, the concepts represented by the indicators of the antecedents 
are the variables. Case study variables, moreover, are typically dichotomous (either A or a; 0 or 1) in nature. The typology of case study units of analysis is contained in table 7.

Table 7. Unit of Analysis Typology in the Case study Approach

\begin{tabular}{|l|l|l|}
\hline \multicolumn{2}{|c|}{ Case Study Units of Analysis Typology } \\
\hline \multicolumn{1}{|c|}{ Unit of... } & \multicolumn{1}{|c|}{ Methodological Approach } & \multicolumn{1}{c|}{ Source } \\
\hline Observation & Case & $\begin{array}{l}\text { Pennings et al. 1999; } \\
\text { Ragin 1987 }\end{array}$ \\
\hline Explanation & Model/Theory & Ragin 1987 \\
\hline Variation & Indicator Variables/Antecedents & Pennings et al. 1999 \\
\hline Measurement & Value & Pennings et al. 1999 \\
\hline
\end{tabular}

Pennings et al. (1999) state that comparative analysis can lead to a complete explanation of the conditions leading to a phenomenon. While this is ambitious, at a minimum, Pennings et al. (1999) assert that it is possible to understand the circumstances preceding or accompanying the phenomenon. Ragin (1987) asserts that by orienting on variables, the experimental and survey approaches focus on strict causality. On the other hand, by orienting on context, the case approach focuses on when causality is important in defining an outcome.

Causal theories, like the one that is the subject of this dissertation, show the reasons for a social phenomenon (King, Keohane and Verba 1994). They are at once broad, parsimonious, and empirically testable. Causal theories include both a definition and an explanation of the phenomenon. The litmus test for any theory, regardless, is its ability to explain the social world (Dubin 1969). Theories, after all, represent 
metaphorical "nets cast to catch what we call 'the world': to rationalize, to explain, and to master it" (Popper 1959, 37-38).

Kumar (2011) suggests that one of the key differences in the experimental, survey, and case approaches is the importance attached to the concepts of validity and reliability. He infers that these concepts are more accepted and developed in the experimental and survey approaches than in the case study approach. This argument that somehow qualitative research is more scientifically valid than qualitative research is a pervasive yet contested proposition (Little 1991). Indeed, the literature suggests that the requirement for quantification varies depending on the research goal (Yin 1994; Pennington et al. 1999; Thomas 2011). Therefore, the approach taken is dependent on the outcome desired.

Thomas (2011) suggests that notions of reliability and validity have less meaning in a case study. This is because as a study of one thing, there is an assumption at the outset that a similar inquiry in a different time and place may not result in similar findings. The case approach, moreover, employs component methodologies in which the concepts of validity and reliability play differing roles (Thomas 2011). Kumar (2011) recommends using alternative criteria such as credibility, transferability, dependability, and confirmability to judge the quality of case studies.

Ragin (1987) advises that the holistic and interpretative nature of the case approach makes it particularly well suited to theory building. One of the principle weaknesses of this approach is the tendency towards particularization (Ragin 1987). It is possible to ensure that research is holistic and interpretative while guarding against particularization through the rigorous application of the concepts of reliability and 
validity. Yin (1994) and Pennings et al. (1999) effectively argue, furthermore, that it is possible to achieve reliability and validity in a case oriented approach.

Kumar (2011) defines reliability as "the ability of a research instrument to provide similar results when used repeatedly under similar conditions" (396). Reliability represents the presence or absence of measurement errors. That is, the degree to which the empirical observation corresponds with the actual conditions. Kumar (2011) asserts that reliability relates to the consistency and stability of the research approach. Pennings et al. (1999) defines the case study protocol as the research instrument in the case oriented approach to social sciences research.

Yin (1994) supports this contention by asserting that reliability is possible with well-documented research procedures. Documentation is possible in any number of mediums. It is possible for later researchers to follow the same protocol and replicate the findings and conclusions of the earlier researcher. Reliability is dependent, therefore, on the protocol and data base design (Pennings et al. 1999). The achievement of reliability occurs through a rigorous and methodical approach to data collection (King et al. 1994). The case study approach is, therefore, an inherently reliable research methodology if applied in the correct manner.

In addition to being a reliable research methodology, the case study approach is valid. Kumar (2011) suggests, "Validity refers to the appropriateness of each step in finding out what you set out to" (402). Validity is the degree to which indicator variables represent theoretical concepts. Kumar (2011) states that validity relates to the appropriateness and accuracy of the research approach. That it is impossible to prove a theory valid is a basic tenet of science. It is possible to gain confidence in the validity of a 
theory, however, by failing to prove it false. The greater the efforts succeed in failing to prove it false, the greater the confidence in the validity of a theory.

There are three types of validity present in this research: construct, internal, and external (Kumar 2011). Construct validity measures the extent to which the research tools are measuring the construct it is claiming to measure. In this dissertation, the construct is public sector innovation. The greater the variance attributable to the construct, the higher the validity of the instrument (Kumar 2011). Yin (1994) asserts that construct validity is possible with multiple sources, peer review, and a clear chain of evidence. The achievement of construct validity, in other words, occurs through sound construction and data collection methodologies.

Internal validity attempts to answer the question of whether it is possible to attribute the observed effects to the variable (Myer et al. 2010). The achievement of internal validity occurs in explanatory studies by establishing "a relationship, whereby certain conditions are shown to lead to other conditions, as distinguished from spurious relationships" (Yin 1994, 33). Yin (1994) writes that internal validity is possible through pattern matching and explanation building. In other words, by focusing on proper data analysis this dissertation establishes the validity its findings, conclusions, and recommendations.

External validity "refers to the extent to which the inferences that are drawn can be generalized to other conditions or populations" (Myer et al. 2010, 16). Yin (1994) suggests that external validity is possible through the logic of replication. It is, in other words, the outcome of sound research design. Yin (1994) asserts that the test for external validity involves "establishing the domain to which the study's findings can be 
generalized" (33). A review of the existing research literature, therefore, centers the evaluation of the research design.

Yin (1994) suggests this is possible because "case-studies, like experiments, are generalizable to theoretical propositions and not to populations" (10). An analytic generalization, and not statistical generalization, is the goal of the case approach (Yin 1994). Yin (1994) claims that in the case approach, analytical generalization is possible by logically linking case data to theoretical propositions. According to his reasoning, theory serves as a template with which to compare empirical results from multiple cases. If two or more cases support the theory it is possible to consider the theory valid. This section provides a concise explanation for the utility of the case study approach in the context of this dissertations theory building exercise.

\section{Tools, Techniques, and Procedures}

This dissertation explores public sector innovation through the lens of three unique cases. This number of cases is a measured compromise between the tyranny of limited resources and profligate rigor. Beck (1977) observes, "The improbability of using a single case to prove a hypothesis is widely accepted" (691). Johnson and Joslyn (1995) assert that a study design based on multiple cases has more explanatory power since it allows for repeated observation of the phenomenon. Bock (1977) goes on to postulate that "to prepare five or ten rigorously comparative cases, when it is possible at all, is a demanding, time-consuming effort: one too extravagant (for both tester and reader)" (691). He postulates that this is especially the case with "small-gauge propositions" like 
public sector innovation (Bock 1977, 691). Therefore, three cases is an adequate number to support theory building.

The selection of the cases for this study was purposeful. So was the method for selecting the participating individuals. The order, collection, coding, measurement, and analysis of the data also occurred according to specific criteria arrived upon deliberately. This all happened prior to the commencement of the field research phase of the study. This section provides a description of the process used to identify and develop the cases. It includes a description of the case screening and selection process. The sections that follow provide a detailed explanation of the tools, techniques, and procedures employed to construct the PSIOI and the OAPSI.

Johnson and Joslyn (1995) assert that the "careful observation of a small number of cases may suggest possible general explanation for the behavior or attributes that are observed" (144). The subject of each case was a specific program or project that meets the definition of innovation. These cases represent specific and discrete examples of systemic behavioral change that results from practices imbedded in institutions. In each case, there was a perception among the participants that these projects or programs both outperformed previous practices and resulted in the common good.

Selecting appropriate cases is a critical first step in gaining a deeper understanding of causal processes (Johnson and Joslyn 1995). The cases were all oriented around patterns of behavior and practices that resulted in innovation. In each case, leaders deliberately sought to embed new practices in the organization although the individual details of each case differed. In one case, the innovation revolved around the port providing facilities for incubating a nascent industry. Another involved building 
organizational capacity in allied governmental institutions. A third involved building organizational capacity to resolve conflicting community interests. These cases involved institutions, industries, and interests separate and apart from those the port traditionally supported.

This dissertation is an exercise in theory creation. For this reason, the choice of cases was specific. In all instances, public sector innovation was present (Ragin 1987; Yin 1994). Figure 8 illustrates the criteria for case selection. The cases selected were from a pool of instances in which there was a correspondingly high PSIOI and OAPSI rating. In other words, from the population of cases found in the upper left hand quadrant of figure 8. In this quadrant, the model suggests that there is an expectation that innovation will occur and that innovation actually occurs.

Figure 8. Comparative Case Selection Criteria

\begin{tabular}{|l|c|lc|c|}
\hline \multicolumn{2}{|c|}{ Measure } & \multicolumn{3}{|c|}{ PSIOI (Expected Value) } \\
\cline { 3 - 5 } & High & & \\
& $(+)$ & High & (Cases Selected) & (No Cases Selected) \\
OAPSI & & & \\
$\begin{array}{l}\text { Rating } \\
\text { (Actual } \\
\text { Value) }\end{array}$ & Low & & \\
\cline { 3 - 5 } & High & & \\
& $(-)$ & (No Cases Selected) & (No Cases Selected) \\
& Low & & \\
\end{tabular}

This dissertation excludes deviant cases in which the phenomenon manifests in a manner that diverges from what the theory suggests. This would suggest other ways in 
which innovation comes about. This dissertation also excluded cases in which the phenomenon's failure to manifest was consistent with the theory. Excluding these types of outcomes is not to suggest they lack value. Johnson and Joslyn (1995) assert that it is possible to learn a great deal by systematically observing these types of cases. They are reserved, however, for future studies seeking to rigorous test this theory. In other words, the study of deviant cases and those in which the phenomenon fails to manifest - while valuable - is beyond the scope of this study.

The following sections of this chapter outline the methodology used to formulate the expected values associated with the PSIOI and the actual value associated with the OAPSI rating. The intent of these indices is to provide a one-dimensional summary of public sector innovation. This approach assumes a relationship exists between the PSIOI and OAPSI summary variables that has explanatory and predictive power. It further presumes the summary variable is more meaningful than any single variable that composes the PSIOI and OAPSI.

One of the premises of this dissertation is that similar values of PSIOI and OAPSI infer the theory is valid. In other words, the PSIOI and the OAPSI rating represent two sides of the same metaphorical coin. In this regard, PSIOI signifies an expected value and OAPSI characterizes an actual value of the phenomenon. As such, the model suggests the same relative scalar results for both PSIOI and OAPSI will occur if the theory is valid. If this is the case, conditions 3A, 3B, or 3C depicted in figure 9 will occur. Conversely, conditions $1 \mathrm{~A}, 1 \mathrm{~B}, 2 \mathrm{~A}$, and $2 \mathrm{~B}$ in figure 9 represent conditions in which the relative scalar results vary by a significant margin and suggests the model is not valid. 
Figure 9: Method of PSIOI/OAPSI Comparative Analysis

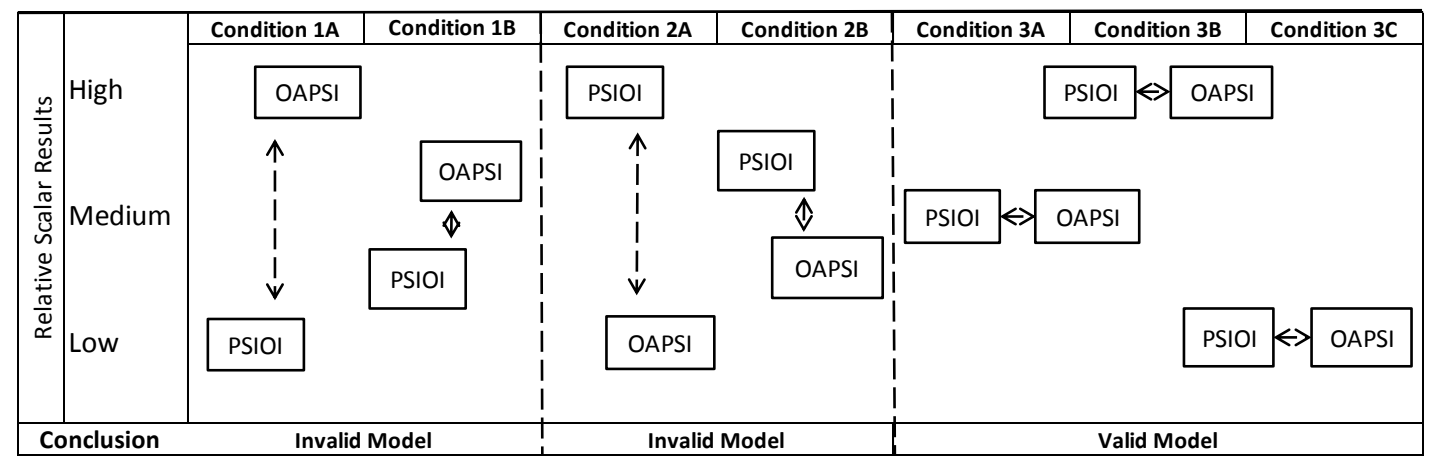

\section{Data Collection and Instrumentation}

This study seeks to define the pathway through which the antecedents operate to open the space for innovation at the nexus of leadership, the organization, and the customer or client. The interview methodology allows for the necessary range of flexibility in studying social phenomenon (Kumar 2011). It is also equally useful in descriptive, explanatory, and causal research (Singleton and Straits 2010). The study of organizational innovation within the unique historical, political, and organizational context of public port authorities in Oregon and Washington State provides additional insight into the theory and practice of innovation in the public sector.

This study employs cluster sampling and interviewing as the data collection methodology for creating the OAPSI rating. Sampling occurs for the roles and relationships integral to the strategic dimensions of the public sector organizational innovation model. Since the assignment of roles is to individuals — and relationships occur between individuals - the individuals in these roles and relationships are the 
appropriate sampling units (Davis and Ingle 2013). Individuals from the port community are the object of this interview effort.

The various social artifacts of public sector innovation can serve to suggest the presence of the requisite sampling units. In applying cluster sampling the primary sampling units are public maritime port communities in Oregon and Washington. The secondary sampling unit represents the individuals discussed above. Sampling units in a fixed geographic boundary provide an optimally efficient approach (Kumar 2011). It is possible to expect to see small differences between heterogeneous clusters (Singleton and Straits 2010).

A purposeful selection of a sample of individuals involved in co-producing public sector innovation should provide an accurate measure of this phenomenon. This is the case since the primary consideration in this sampling design is an informed judgment as to which individuals are best suited to provide the information necessary to achieve the study's objective. This research design selected only those individuals most likely to possess relevant information. Those selected were also willing to share information regarding their actions and opinions.

In-person interviews with individuals in roles and relationships that relate to the co-production of public sector innovation in the public marine port environment occurred during the field research phase of this dissertation. In the context of this research project, innovation manifests as the following artifacts in an organization:

1. Introducing new policies, processes, services, or programs

2. Changes to existing processes, services, or programs (i.e. extensions, redesigns, etc.)

3. Serving a new client base 
These are the forms of innovation and they represent dependent discrete events in this study. Conceptualizing and identifying innovation in this manner avoids the ambiguity inherent in organizational settings. This allows for the comparison of innovation on a case-by-case basis.

The primary method of data collection was through a semi-structured face-to-face interview process. While there is a limit to the scope of a semi-structured interview, the interviewer used discretion in selecting interview questions that met the specific objectives of this study (Singleton and Straits 2010). This method offers greater flexibility in the format and type of questions employed. The advantage of this approach is in providing the interviewer the opportunity to clarify questions and elicit additional responses (Singleton and Straits 2010).

This method allows for a rich understanding of the social phenomenon under study because it permits for the introduction of complex questions. Face-to-face interviews also allow for the easy alteration of the interview once it has begun (Kumar 2011). This methodology permits an extended investigation of the concepts, features, variables, or explanatory factors that underlie the model. While this approach will improve response rates, interviews are very expensive and time consuming to conduct. Interviews can also introduce interviewer bias. The design of this interview process, moreover, is cross sectional.

Interview subjects included individuals throughout the various levels of Oregon and Washington State port authorities. A standard battery of questions with associated follow-up questions allowed for the elicitation of individual experiences, perceptions, opinions, feelings, and knowledge related to innovation. Interviews yielded valuable 
direct quotations related to innovation. Interviews represented the primary methodology for data gathering. Stratified random and cluster sampling was used to increase the level of confidence necessary to support generalization.

The specific number of cases selected for this dissertation depended on the resources available at the time of the study. The specific number of individuals selected for interviews depended on the number of individuals that had relevant knowledge. Relevant knowledge included experience related to innovation in the public sector and specifically information with the innovation that was the subject of the case. Established research protocols involving informed consent and the protection of human subjects insured the maintenance of ethical standards and confidentially.

The strategic dimensions of public sector organizational innovation posit that a dynamic interrelationship exists between leadership, the customer or client, and the organization. Public sector innovation results from co-production. This occurs when the antecedents that the model suggests operate through a discrete pathway within specified domains. This meant that interview questions served to identify each of these antecedents. In other words, the questions tie directly to the discrete variable. Overlapping questions served to explore this complex topic (Singleton and Straits 2010). Appendix A contains the sample interview guide and interview instrument. It contains open-ended, free-response questions. These questions require individuals to respond in their own words (Singleton and Straits 2010). The interview instrument also lists several follow up questions to probe for responses, as necessary, to ensure clarity. These questions, and any follow up questions required of the interviewer, were as direct as possible. In addition, there was a standard battery of individual and organizational 
demographic questions. All questions were pretested and revised as appropriate for clarity, consistency, and meaning (Kumar 2011).

Figure 10 presents a graphical representation of the strategy for collecting data. The next sections of this chapter provide more detail on the data collection process as it relates to the construction of the PSIOI and the OAPSI rating. In addition to the primary sources of data, the dissertation used two kinds of secondary data: field observations and document collection (Singleton and Straits 2010). Field observations of participants occurred in conjunction with the interviews along with participant observations. Both further validated the interview results.

\section{Figure 10. Dissertation Data Collection Methodology}

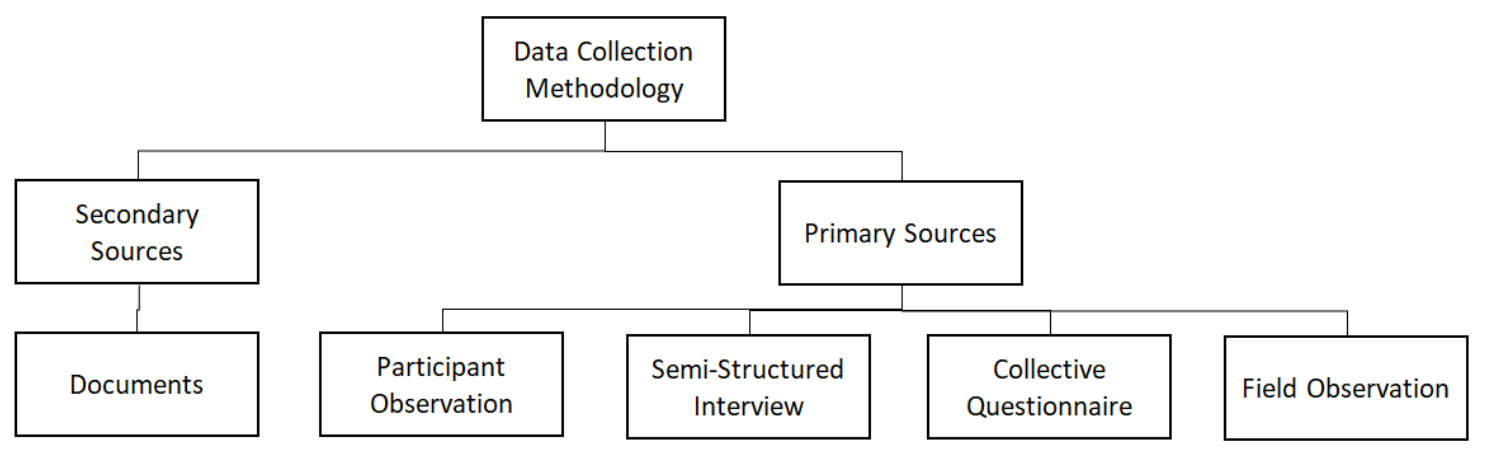

(Adapted from: Kumar 2011)

Field and participant observations included descriptions of behaviors, activities, and actions (Kuman 2011). These included any aspect of observable human experiences related to innovation. The intent was to gather insight into the deeper social construct that was operative in the public sector. These reveal themselves through interpersonal interactions conditioned on organizational or community processes. The data consisted of 
field notes that were rich in detailed descriptions. The observations provided useful context within which the interviews occurred.

The final type of qualitative data was contained in documents collected in conjunction with the interviews and field observations. These included documents created by both public and private sources (Singleton and Straits 2010). Written material represents a rich source of data. It includes documents from organizational or program records. This included memoranda and correspondences, official publications, government reports, letters, artistic work, photos, and memorabilia (Patton 2002). To support reproducibility this study only used publicly available documents. This included documents in the public domain and easily accessible online or in public archives. For this study, public documents were those subject to FOIA disclosure.

The challenge with using documentary data is to preserve a reasonable premise of participant confidentiality while capturing the written record in a way that preserves its context and meaning. Context is key to understanding the phenomenon of co-created public sector innovation since it relates to the intent of the author and the reason behind a documents creation (George and Bennett 2005). Understanding context is fundamental to documentary analysis. The research effort in this dissertation made no effort to exclude "gray" documents from collection or analysis. The fact that these were outward-facing agency documents could bias their content.

There are some weaknesses inherent in this approach to data collection. This includes, for example, the tendency of interview respondents to provide socially acceptable answers (Singleton and Straits 2010). Singleton and Straits (2010) suggest that "order effects can occur when respondents invoke the conversation principle of non- 
redundancy to answer similar questions differently" (328). There is also an intrinsic weakness in relying on reported rather than observed behavior.

There is a concern that this data collection approach is susceptible to reactivity resulting in the subsequent introduction of systematic measurement errors (Kumar 2011). There is also the danger of a variety of measurement errors ranging from a simple lack of question understanding and difficulty recalling past events, to unstable attitudes and opinions, or even a lack of truthfulness (Singleton and Straits 2010). It is possible to partially control for interviewer error through standardized interviewing. It is difficult, nevertheless, to fully control for these potential errors.

\section{Public Sector Innovation Organization Index (PSIOI) Formulation}

The purpose of this section, and those that follow, is to provide future researchers a metaphorical roadmap to replicating this study. This roadmap will provide the transparency necessary to analyze the research and evaluate the conclusions. This section, for example, on PSIOI formation describes the process used in this study to formulate the interview protocol and the data-gathering instrument. This section also reviews the protocol for screening and selecting the interview subjects. Finally, this section touches upon the wording, form, order, collection, coding, measurement, and data analysis protocols.

Indices allow for the presentation of a single representative value of a complex or complicated phenomenon - in this instance, public sector innovation (Johnson and Joslyn 1995). This section outlines the tools, techniques, and procedures used to formulate the Public Sector Innovation Organizational Index (PSIOI). The first level of analysis 
performed on a potential case was the PSIOI. Cases selected for further development and analysis had a PSIOI greater than one (PSIOI > 1.0). Instances where the PSIOI was less than one $(\mathrm{PSIOI}<1.0)$ were not included in this study.

The PSIOI represents a value of innovation a researcher would expect to observe in the organization. As such, the primary use of the PSIOI was to screen and select individual cases for inclusion in this study. The PSIOI was constructed using secondary data. Empirical observations of publicly available records form the basis of the PSIOI. These secondary source documents were available either electronically or in print. To ensure transparency and duplicability of the results, this research effort relied upon only publicly available documents. There were no requests for documents through the Freedom of Information Act (FOIA). While outward-facing agency documents could have a bias in their content, a FOIA request may yield additional or contradictory information.

The co-production of public sector innovation is a complex, social, and multidimensional phenomenon. As such, care is required in devising a measurement strategy. Johnson and Joslyn (1995) advise that using an index will allow the researcher to "capture numerous aspects of a complex phenomenon" (Johnson and Joslyn 1995, 96). The index makes this possible by "representing the existence of that phenomenon in particular cases with a single representative value" (Johnson and Joslyn 1995, 96). For these reasons, this dissertation employed indices.

The Public Sector Innovation Organization Index (PSIOI) represents an attempt to create such an index. This approach involved the creation of a multi-item index that accumulated scores on individual elements. The summation of which expressed a 
composite measure of the phenomenon. The higher the index, the greater the level of innovation expected in the organization. This approach is not without risks. Principle among them is identifying the most relevant and impactful variables (Kumar 2011). In other words, separating the metaphorical wheat from the chaff.

The PSIOI represents an attempt to simplify the presentation of an extensive amount of secondary source data. It also allows for an exploration of the dimensionality of measures through the construction of a multi-item scale and the use of an index. The synthetization of data in this manner allows the researcher to observe both the broad arch of the data and the specificity of a narrow slice of the data. Johnson and Joslyn (1995) suggest this measurement strategy enhances both the accuracy and precision of the measurement. This is the case because this approach has the benefit of classifying observations in terms of attributes on multiple variables.

The PSIOI is a multi-item measure. It represents the combination of values across several independent variables to form a summary measure of expected innovation. The PSIOI reflects the measurement of the dependent variable of expected innovation. This index is the product of an additive process in which there is an equal weight assigned to each independent variable used to calculate the score. Differing independent variables serve to operationalize the antecedents. These antecedents inhabit the model's three core theoretical constructs or domains.

This approach mitigates the risk of uncertainty surrounding the salience of the antecedents in co-producing public sector innovation. The level of measurement employed in this study suggests the meaning that it is possible to derive through the assignment of values to empirical observations (Johnson and Joslyn 1995). It attaches to 
the variable and represents a claim to the precision inherent in the measurements. The level of measurement provides a reference to the type of information it contains. It also makes a claim to the kind of comparisons it is possible to make across multiple observations.

Operationalizing organizational innovation required the assignment of a numeric value to a variable at the time the data was coded (Kumar 2011). Kumar (2011) refers to this process as coding. In the context of this case study, coding involved reviewing an extensive amount of primary and secondary source documents to assign numeric values to differing variables. Concurrent with developing the interview questions, a codebook listing the set of numerical values assigned to each independent variable was developed Appendix B contains the codebook for this study.

The data for the construction of this index came from sources specific to the individual organizations involved. The techniques used for mining the data were nearly identical in each of the three cases. If this was not the case, an annotation accompanied the analysis explaining any differences in data collection between the cases. There was a deliberate attempt to limit the collection and coding of documents to those issued or created within twelve months of the start of the data collection process for the individual case to ensure data currency.

The documents collected, cataloged, coded, and analyzed were available online or accessible through the Portland State University library system. The secondary source documents generally fell into one of six categories: marine, industrial, environmental, community, news or media releases, and the port's commission. Selecting documents in these six categories was a control measure. The intent of which was to support, to the 
extent possible, the derivation of a consistent method for measuring PSIOI across different ports.

Searching publically available websites required the use of key search terms related to pubic marine ports in the Pacific Northwest. Six search terms were subsequently developed. The six included marine operations, industrial properties, environmental, community outreach, news or media releases, and the port commission. Previous knowledge of public marine ports and special district operations supported the development of these terms. The document searches started with these terms; however, searches also involved derivatives of these terms. The search terms also served as convenient categories of documents. These six categories were also useful in cataloging the material used in the analysis necessary to create the PSIOI.

The assignment of the values to create the individual PSIOI's was within the context of the attributes of resilience, quality, accessibility, and efficiency. Special districts characterize an institutional form that exists at the intersection of a parochial scope of interests and the political arbitration of values (Morgan et al. 2013). The attributes of resilience, quality, accessibility, and efficiency closely align with the special district institutional form. This was the reason for the selection of these attributes. Resilience, quality, accessibility, and efficiency also have the benefit of serving as a bridge between the NPM and NPG paradigms discussed earlier.

This study employed an ordinal typology of measurement to arrive at the value assigned to both the PSIOI and the OAPSI. This approach assumed that it is conceivable to measure more or less of a variable. This suggests that it is possible to make comparisons between multiple independent observations. In this regard, "the intervals 
between the numbers have no meaning; all that matters is that the higher numbers represent more of the attribute than lower numbers do" (Johnson and Joslyn 1995, 96). This approach is useful in theory building to the extent it supports the identification of salient variables.

The rank ordering of the measurements is from "high" to "medium" to "low" such that positive or plus one $(+1)$ represents a high measurement, zero $(0)$ reflects a medium measurement, and negative or minus one (-1) is a low measurement. A high measurement with regard to secondary source document analysis is one in which there were three or more positive references to an independent variable. Pre-testing of the PSIOI established this as an adequate baseline threshold for determining a robust measure of the independent variable for screening purposes. A medium measurement represents between one and two positive references. Pre-testing of the PSIOI established this as an adequate baseline for determining the presence of the independent variable as well.

A low measurement is either a negative reference or the absence of any references to a variable. Negative and positive references around an independent variable offset or cancel each other on a quid quo pro basis. In the construction of this measurement strategy, the assignment of the zero point is such that it does not represent the absence of the independent variable. The intent behind the selection of this ordinal scale was to provide linearity, reliability, validity, and reproducibility in the measuring devise (Freeman 1977). There was no effort to judge the quality of the measurement, only the quantity.

The objective of content analysis is to transform verbal and non-quantitative publicly available records into quantitative data for use in creating the PSIOI (Johnson 
and Joslyn 1995). Many of the documents accessed for this dissertation were located on publicly accessible websites. The port associations to which a port belongs or other governmental or quasi-governmental entities at the state and/or federal level maintained the majority of these websites. Publicly available online media sources like local newspapers was also a source of secondary data.

Content analysis for this dissertation involved coding and notating each instance of a reference to the quality, efficiency, resiliency, or accessibility of a particular independent variable. There were instances in which a single reference related to multiple categories. In these instances, a single reference received a single coding notation. Coded was for the most appropriate reference term. It was possible, however, for a single document to contain multiple distinctive reference terms. Whenever possible the evaluation and analysis took into account known institutional or organizational biases. There was, in other words, a contextualization of the content analysis.

Figure 11 depicts the approach to selecting cases for this study using the PSIOI methodology. The selection of the population of potential ports for inclusion in this study occurred through an examination of the membership rolls of the public port associations of the states of Washington and Oregon. The result was the port population depicted in figure 11. Selection of cases in this manner had the effect of limiting the population of ports to United States domestic institutions in the Pacific Northwest. Study economics dictated the geographic limitation of this dissertation.

The role of the Washington Public Ports Association (WPPA) and the Oregon Public Ports Association (OPPA) is to promote the interests of the port community. These associations seek to accomplish this through the maintenance of effective government 
relations, ongoing legislative education, and strong public advocacy programs. They conduct these activities on behalf of their members in their respective states and at the national level. Membership in these associations represents an ongoing interest, commitment, and involvement in the port community that could align with involvement in innovation.

\section{Figure 11. PSIOI Case Selection Methodology}

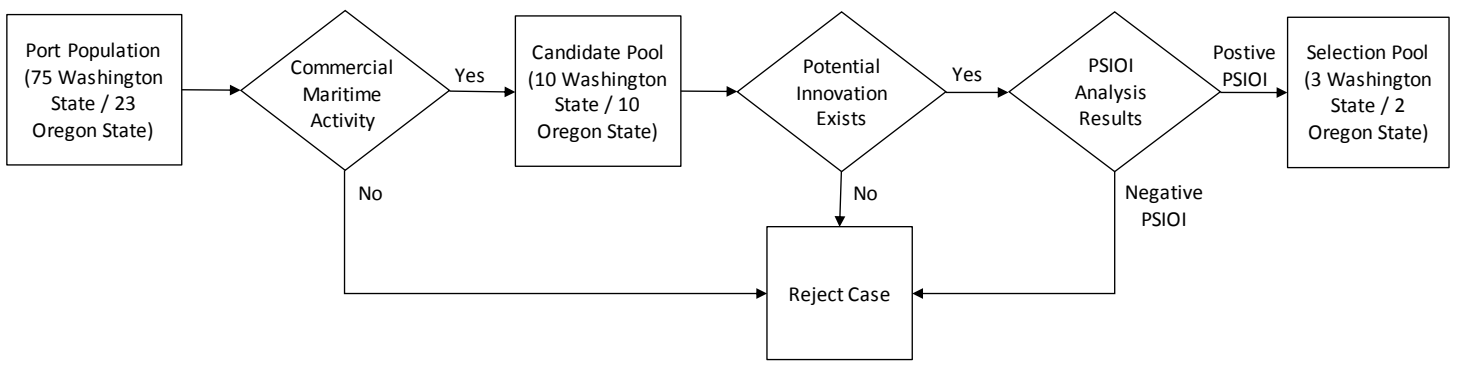

The population of ports considered for this study consisted of seventy-five ports located in Washington State and twenty-three in Oregon State. Ports that did not participate in commercial maritime related activities were not included in this study. This was the case because they could distort the results since these special district ports tended to engage exclusively in commercial real estate development. This resulted in the candidate pool depicted in figure 11. Ports in Oregon and Washington state conduct a wide range of activities designed to foster economic development. This includes supporting public recreation, commercial fishing, industrial real estate development, and general or commercial aviation. 
The intent of the purposefully limitation of the study to special districts engaged in commercial maritime activities was to ensure the existence of a similarly constituted set of organizations. This was an attempt to control for random variation between cases resulting from the asymmetrical production specialization of the organization. Participation in other industries (e.g. recreation, commercial fishing, industrial real estate development, or aviation) did not prelude a port from inclusion in the study. The result of this screening was a candidate pool of twenty ports in Washington and Oregon, split equally between the states.

There was an analysis conducted on each of the twenty ports in the candidate pool using the PSIOI methodology. This resulted in the selection pool depicted in figure 11. This involved creating a PSIOI for each port while concurrently screening the port for innovations. Ports that had an innovation and a positive PSIOI were included in the selection pool. Five ports met the criteria of a positive PSIOI. Two of these five ports were involved in the same innovation. To control for any extraneous variables associated with urbanization, the three cases selected were a port district in an urban area, a mixed urban and rural district, and a rural district.

\section{Organizational Antecedents of Public Sector Innovation (OAPSI) Formulation}

This section outlines the tools, techniques, and procedures used to formulate the OAPSI rating. This index represents an actual value of innovation observed in the organization. The data used to construct this rating largely derives from primary sources. The empirical observations used to formulate the OAPSI resulted predominantly from structured interviews. Similar to the measurement strategy used in creating the PSIOI, the 
OAPSI uses a simple additive scale to represent an aggregation of the various dimensions of this complex phenomenon. What follows is an explanation of the methodology approach used to create the OAPSI rating. This dissertation employs a purposive approach to selecting interview subjects. This is a nonprobability sampling technique, the purpose of which is to draw individuals with the relevant knowledge of, and experience with, the phenomenon into the study (Freeman 1977). This is a separate and distinct process from the case study selection process.

Freeman (1977) suggests nonprobability sampling is appropriate to "handle certain special research tasks," especially in situations where the intent is to match the dependent variables of innovation across all cases (231). The aim, therefore, was to draw into the study the relevant ports and persons that will serve to facilitate empirical understanding and prediction that is capable of generalization. This is only possible if the model is capable of summarizing relationships between observed facts that communicate general knowledge (Johnson and Joslyn 1995).

There was a deliberate alignment of the study protocol with a level of effort appropriate to the dissertation - in terms of time, convenience, and costs. This involved taking deliberate care and precision in the conception of the research protocol. This was also the case with the creation of the research instrument. What follows is an overview and discussion of the wording, form, order, collection, coding, measurement, and analysis of the interview questions. Control for bias and error in the interview protocol and the interview instrument primarily occurred through a pre-testing protocol outlined below.

The interview process occurred in three phases: screening, interview, and followup. The screening process resulted in the creation of a pool of potential interview 
subjects. Individuals in the pool became candidates for selection in the interview phase. The follow-up phase occurred after the completion of the interview phase on an 'as needed' basis. The first step in the screening process was to make inquiries of potential participants. Generally, the first contact was with subjects known personally or professional to the researcher, although this was not always the case. In some instances, subjects recommended other subjects for inclusion in the study. This was often the result of their understanding of the research study gained during the screening process. In other instances, individuals in the public port community nominated subjects for inclusion in the study.

The screening process involved providing each participant a brief general description of the research. This provided potential subjects a contextual understanding of the project. A series of open-ended questions determined the potential candidate's level of participation and understanding of the specific innovation under study. Included in the study pool were subjects articulating an understanding or involvement in the specific innovation that was the focus of the case. The interview phase began once an individual entered the subject pool. This was generally at the conclusion of the screening interview.

The second phase of the process involved conducting the actual interview, a oneon-one interaction between the subject and the researcher. The interviews occurred in a private setting; generally, in a conference room or private office at the port. The intent was to provide the anonymity necessary to elicit candid comments and allow free expression in responding to the questions. The third and final phase of the interview process was the follow-up. Follow-up interviews were infrequent. They typically 
occurred when a question arose over a subject's response and it was subsequently necessary to clarify the meaning or intent behind the answer.

The goal of each question was to elicit data on a specific measure of an independent variable that served to operationalize an antecedent of innovation. Each question, therefore, related to the measure of only one independent variable. As such, the purpose of each question was to provide a measure of a discrete independent variable. Each question, consequently, incorporated only one thought. There was deliberate care taken in objectively wording each question in a manner that avoided the connotation of emotional bias or double meanings. In other words, the intent was to design emotionally neutral questions (Kumar 2011).

The wording of each question was short, direct, and non-leading. This was to ensure ease of comprehension. It also aided subject retention when answering the question (Freeman 1977). To the extent possible, a screening process led to the elimination of overly academic language that were unnecessarily technical or specialized. There was a risk to this approach. Short and direct questions may lack the clarity of longer more explanative questions. Pretesting activities provided valuable in "debugging" the questions (Freeman 1977, 234). The process provided important insight into the reliability and validity of the questions prior to the commencement of the fieldwork.

The interview instrument consisted of open-ended questions in which the subject responded in their own words to the question asked. The open-ended question format takes into account the complex, multifaceted, and interdimensional nature of the phenomenon (Johnson and Joslyn 1995). This form of inquiry allows for a detailed description and, if necessary, probing follow up questions. Probing ensured a full 
response to the question. It was also a helpful approach when the response to the question required additional clarification. This form of data gathering required special care to ensure that extemporaneous probing or follow-up questions did not contain leading or bias statements (Johnson and Joslyn 1995). Appendix A contains the primary source data collection instrument or interview guide.

Uni-dimensionality or homogeneity was a consideration in the question design (Freeman 1977). Each question provided a measure of only one quality or dimension of the behavior under study. The behavior was the basis for the empirical observation of the independent variable. The open-ended approach to interviewing has the advantage of allowing for a detailed reply that contained subtle nuances, revealed intensity, and exposed affective content (Freeman 1977). Open-ended questions also had the benefit of providing rich, organic detail. This form of inquiry allowed for robust responses and the exploration of the contextual nature of the phenomenon.

The difficult and time-consuming analysis requirement inherent in these types of questions offsets this advantage (Freeman 1977). This is because the judgmental process necessary for proper content analysis proceeds according to formulated rules and systemic procedures (Holsti 1977). The contextual nature of this social phenomenon creates challenges in objectively coding responses. This can lead to issues over proper contextual interpretation of the data. Another challenge with this approach is the danger that the probes may contain leading questions (Freeman 1977).

There is a nuance to the open-ended questioning technique. At the conclusion of the open-ended question, the subject received a closed-ended question. This design allowed for the discrete summarization of the subject's perception of the presence or 
absence of the variable. In this regard, each subject rated the presence of the variable. The purpose of this approach was to provide an additional measure of insight into the subject's perception of the phenomenon. This makes the approach a sort of hybrid between open- and closed-ended questions. Appendix A contains the data collection instrument and Appendix B encompasses the case content coding book used in the construction of the OAPSI rating.

The general rule for the presentation of the interview questions was homogeneity (Johnson and Joslyn 1995). For example, the presentation of the questions related to the organization appeared together in sequence. The question order proceeded from the general to complex whenever possible. There was also consideration given to the impact of the previous question on the subject, although this was not a major factor. This is the case since the way question grouping occurred had the potential for supporting memory recall. There was also consideration given to avoiding redundancy, maintaining consistency, and insuring saliency in the questioning (Johnson and Joslyn 1995). The intent of these measures was to reduce the effects of question fatigue on the subjects.

Content analysis is the process of converting verbal and other non-quantitative records into quantitative data (Johnson and Joslyn 1995). Content analysis involved assigning an empirical measure to each independent variable. Content analysis of the individual interviews resulted in the formulation of the OAPSI rating. One of the purposes of this section, and the preceding one, is to enhance the validity of the content analysis by providing a precise explanation of the tools, techniques, procedures, and content categories used in this analysis. 
Holsti (1977) advises that, "The most common weakness of studies using an a priori standard stems from the absence of a clearly defined basis for judgement" (295). To combat this potential flaw, the categories of content measured and their values appear in the case content coding book. The definitions are contained in Appendix B. This codebook contains a detailed description of the measurements associated with each indicator. Indicators serve to operationalize the concept of an antecedent. The codebook also links the antecedents with the core theoretical constructs or domains of the model.

The codebook contains content categories that are salient, exhaustive, independent, mutually exclusive, and derived from a single classification principle (Jones 1985). The recording unit for each measurement is an independent variable. The independent variables represent particular subjects or the expression of ideas. The content analysis links the working definition of salient independent variables to indicators. A measurement consists of the independent variable and the scaler value attached to the independent variable. The working definition of each independent variable is contained in the case content analysis coding book found in Appendix B. This involved the use of an ordinal ranking scale. Having only one individual perform the coding enhanced intercoder reliability.

The assignment of values within the rubric of the OAPSI rating was consistent with the measurement strategy outlined in the previous section. It is from "high" to "medium" to "low" such that positive or plus one $(+1)$ represents a high measurement, zero (0) reflects a medium measurement, and negative or minus one (-1) is a low measurement. A high measurement as one in which there was a robust or particularly noteworthy presence of the independent variable. A medium measurement represented a 
minimal or the negligible presence of an independent variable. In other words, a trace amount exists. A low measurement as the complete absence of the independent variable. Similar to the PSIOI, there was an arbitrary assignment of the zero point such that it does not represent the absence of the independent variable. No value was assigned in instances were the subject was unable to answer the question.

An evaluation of the validity, reliability, and reproducibility of the interview questions occurred at three key points. These points were during the pre-testing of the interview questions, at the end of the first case study, and after the preliminary analysis of the fieldwork. Validity, in the context of the study questions, reflects the extent to which the values appeared to approximate the true values (Kumar 2011). In this regard, there was an evaluation of the face, construct, and content validity of the questions. Therefore, for example, the presence of an antecedent in the occurrence of innovation provides some assurance that a measure of construct validity exists (Johnson and Joslyn 1995).

The same holds true for face and content validity. This assumes that the instrument is measuring all relevant aspects of the domains under study using the measurement technique outlined in this chapter (Johnson and Joslyn 1995). The evaluation of a question's reliability was such that over the duration of this study, the subject's responses produced similar results (Kumar 2011). Concerning reproducibility, the evaluation of the measurements related to the ability to derive discrete values that were consistent in measurement over the duration of this study and between the various subjects (Freeman 1977).

Severl steps ensured the protection of the confidentiality of subjects and their respective organizations. The interviews for the case studies occurred in a private setting 
selected by the subject. Initial contact with the subjects occurred by phone or email. The names of the subjects only appear on the IRB approved informed consent form and interview log. These documents remained under lock and key once they contained data. No personally — or organizationally — identifiable information of any kind appears in the transcriptions or summaries of the transcriptions.

In addition to the safeguards noted above, the individual quotes and subject observations were non-attributional. This provided privacy protection to the subjects and served to encourage candid responses. A general description of the organization or individual was included in this dissertation to the extent it was necessary to provide context. If this was the case, only a general description of the individual's role in the organization appeared in this dissertation. In some cases, this involved identifying either the department or level of the individual in the organization. Likewise, only minimal details of the port's operations appear in this dissertation.

\section{Case Study Organization and Layout}

The individual case studies presented in the next chapter begin with a brief introduction of the case before presenting the background and setting for the case. This includes a general overview of the organization that is the subject of this case and the port's leadership structure. This section also includes an approximate geographic location of the port, the ports major lines of business, the governance structure of the port, and the organization of the port. The inclusion of this data is only to the extent that it conveys information necessary to contextualize the case. This section also includes a description and discussion of the nature of the innovation under study. 
The sections that follow present the case study analysis. This involves a detailed examination of the elements and structure of the PSIOI and OAPSI rating. In each of the cases, the creation of the PSIOI preceded the formulation of the OAPSI rating. The analysis in chapter 5 thus proceeds chronology, starting with a discussion of the interpretation of the PSIOI results. This includes details on the sources and data used to construct the PSIOI. The analysis in the PSIOI section is refined and presented using the summary and coding for the measurements of each of the independent variables.

The construction of the PSIOI involved assigning values to the observed levels of quality, efficiency, resiliency, and accessibility of the organization for each of the variables listed below. The PSIOI coded data was publicly available, open source material. The coding for the index occurred independently from the measurements of the OAPSI rating. The intent of creating the PSIOI independently, and in advance of, the OAPSI was to avoid the danger of data cross contamination. There is a tabular summary of the PSIOI coding for each case in the within-case analysis section.

The next section presents the OSPSI. The coded interviews were the source of the OAPSI rating. The analysis of each antecedent continued independently, however, from the evaluation accounted for their interrelated nature. There is sequential presentation of the analysis beginning with the measures of the independent variables and proceeding to the domain of the antecedent. A presentation of the between-case or cross-case analysis occurred in the final section of this chapter. This included a discussion of linkages between antecedents when they emerged.

Each case study involved an analysis of ten independent variables. Each independent variable is an indicator of an antecedent. More than one independent 
variable could serve as an indicator of an antecedent, however, each antecedent had at least one independent variable indicating it presence. The independent variables include:

1. Existence of feedback loops

2. Leading with a vision

3. Resources constraints

4. Co-production awareness gaps

5. Ability to sunset through co-production

6. Information asymmetry

7. Transformational leadership

8. Ability to experiment

9. Incentives for improvement activities

10. Risk-taking preferences

The narrative presented in the OAPSI section of each case contains a discussion of the measurement for each independent variable. Like the PSIOI, there is also a tabular summary of the OAPSI rating presented in the final within-case analysis section of the individual case study.

The final section of chapter 5 details the cross-case analysis undertaken as part of this study. This approach was helpful in identifying and exploring the complex causal links related to this phenomenon. In this regard, the between-case or cross-case analysis generated explanations of the observed phenomenon (Yin 1994). Drawing and verifying conclusions from these differing cases required a systematic understanding of a theoretically significant explanation for the observed outcome. In this regard, the 
elimination of any variables not attributable to an individual case study is possible though systematic evaluation (Yin 2003).

Cross-case analysis is a qualitative methodology widely used in the social sciences (Yin 2004). In analyzing multiple cases, patterns emerged that are associated with a certain outcome-in this case, innovation. This outcome represented the dependent variable. These patterns, furthermore, are associated with the independent variable. They suggested 'how' and 'why' innovation occurred (Yin 1994). Cross-case analysis, furthermore, is akin to replication testing in which the patterns for each case support the validity of the results.

Emergent themes represent topical insights that arose during the analysis of each case. Emergent themes reflect prominent or frequently recurring inflections observed in the data. They represent a snippet of insight taken from the subject's comments, a piece of documentation, a personal thought, or an exhibition. They reflect an idea that is reoccurring or pervasive. Emergent themes represent a subject, focus, or topic with a specific and distinctive quality or characteristic. In this regard, emergent themes are ubiquitous and, therefore, worthy of additional study.

The data-gathering and coding phases captured the manifestation of the emergent themes. They did not appear, however, until the analysis phase of each case study. Each emergent theme attached to the OAPSI rating. The model, however, implies their presence in the PSIOI. Chapter 6 deals with emergent themes. This includes a brief discussion of their possible implications. These topical insights provide a roadmap to future research related to the co-production of public sector innovation. They are valuable in this regard. 


\section{Conclusion}

Chapter 3 presented a model that explains the co-production of public sector innovation. This chapter builds on that information to suggest how to operationalize the model. Operationalizing the theoretical framework served to validate the model through empirical observation. In this regard, ten independent variables served to indicate the presence of the six antecedents that created the pathway to the co-production of public sector innovation. Each of the antecedents ties to a domain that models real world social interactions.

This chapter outlined the process for operationalizing the model. It opened with a presentation of the research strategy, approach, and design used to accomplish this goal. Chapter 4 also outlined the tools, techniques, and procedures used to formulate the PSIOI and the OAPSI rating. These indices measure the dependent variable of public sector innovation. This chapter included the instrumentation used for the empirical observation necessary for data collection and analysis. Additionally, this chapter covered the process of formulating the PSIOI and the OAPSI rating. A brief overview of the organization and a layout of the case study presented in the next chapter concluded this chapter.

Chapter 5, the next chapter, presents the analysis that validates the theory. This chapter contains an analysis of three cases in which the phenomenon is present.

Validating the theoretical framework involved explaining, identifying, and predicting the causal mechanisms that lead to innovation. Chapter 5 presents the within-case analysis and cross-case comparisons that illuminate the interactions of the subjects and allows for the prediction of social outcomes. The findings, conclusions, and recommendations that 
result from the case study analysis are the subject of the sixth and concluding chapter of this dissertation. 


\section{CHAPTER 5: CASE STUDY ANALSIS AND PRESENTATION}

What follows in this chapter is a presentation of the three cases used to validate the model of the strategic dimensions of public sector innovation. The PSIOI and OAPSI methodology presented in the previous chapters is the basis for the analysis of each case. These indices serve to operationalize the model that explains the co-production of public sector innovation. It does so in a manner that allows for an understanding of organizational interactions and the prediction of social outcomes. The PSIOI and OAPSI reflect a measure of the dependent variable of co-produced public sector innovation. This measure is the result of the empirical observation of a number of independent variables that reflect the antecedents that create the pathway to the co-production of public sector innovation.

\section{Introduction}

This chapter contains an analysis of the data collected through empirical observation during the field research phase of this dissertation. The previous chapter concluded with a brief discussion of the organization and layout of the three independent cases undertaken to validate the model that explains and predicts the co-production of public sector innovation. The presentation of the three cases in this chapter was in the chronological sequence of their analysis. The final section of this chapter presents the cross-case analysis. This concluding section of the chapter presents a synthesis of the three cases. 
Each case is presented as an integrated whole. The objective of this chapter is to present the data in an understandable format. To accomplish this, the dissertation takes a narrative approach to presenting each case. Whenever possible, this chapter allows the subjects to speak for themselves. This chapter, therefore, includes quotes that came directly from the subjects to illustrate the nature of the measurement of each independent variable. Using the exact language of the subjects provides authenticity and insight into the perceptions that form the reality of the individuals engaged in the co-production of public sector innovation.

The way the validation data was collected, coded, and analyzed is the main topic of this chapter. These chapter incudes sections, which analyze the approach taken to the field interviews, content coding methodology, and content analysis. This chapter presents the results of the fieldwork and the subsequent analysis of the data. The investigation of the cases proceeded in two stages. The first stage was a within-case analysis. During this stage, the analysis relied upon the data collected to construct the PSIOI and OAPSI rating for the individual cases. The second stage involved conducting a between-cases comparative analysis. The analysis in the second stage involved a comprehensive exploration of all three cases simultaneously. The analysis in these two stages chronicled the pathway through which the antecedents operated to open the space for the coproduction of public sector innovation.

\section{Case 1: Life Sciences Facility}

The first case involves the modification of an existing structure on port property — in this case, from one industrial use to a completely different industrial use. 
The setting for this case is a major metropolitan area. This metro area has a major research hospital and is quickly becoming a center for biomedical research. This case involves a large public port located on a major navigable waterway in the Pacific Northwest. This port is a fixture in the community and it has a history spanning over onehundred years. This public agency has extensive maritime and industrial holdings.

The port's properties stretch from a vibrant downtown business and residential district, through riverfront property that is largely isolated from the community. The maritime holdings include several deep-water facilities capable of handling ocean-going ships and smaller inland river vessels. These facilities are located along a major navigable inland river channel. The port is adjacent to interstate highways and has access to several national Class 1 rail lines. This case focuses on one of the port's terminals located in the downtown district.

\section{Background and Setting.}

A three-member elected commission represents the community's interests at this port. Each commissioner hails from one of three districts that comprise the countywide port district. Geography and population provide the basis for each of the three port districts. Citizens elect the commissioners "at large" within their respective districts. The commissioners hold staggered six-year terms. They are responsible for setting port policy. The commissioners appoint an executive director who reports directly to the commission. The director provides day-to-day leadership for slightly over one-hundred port staff. Executing the commission policies is the task of the executive director and port staff. 
A portion of the port is adjacent to downtown business and residential district in a major urban area. The port refers to this property as the 'Terminal 1' location. This portion of the port property was the original port operated terminal and, therefore, has historical significance. In this respect, it serves to link the community with their maritime heritage. It also holds an important place in the organizational culture of the port. In this regard, it serves as the symbol of the ports origin. The location of this property in the populace downtown business and residential district means that there is a high level of community interest in the port's plans for the property.

One of the long-term tenants at Terminal 1 was a full service, mid-market hotel. A medium-size hotel chain headquartered in the Pacific Northwest operated the facility until recently. The chain built the hotel on land leased from the port. At the termination of the lease, the hotel and fixtures located on the Terminal 1 property reverted to port ownership. The hotel chain notified the port after several decades of occupancy of their intent to vacate the premises. The port pursued using the cluster of hotel rooms to create a life sciences complex.

Public Sector Innovation Organizational Index (PSIOI).

This section contains a review of the documents collected, coded, and analyzed in the development of the PSIOI. Table 8 (see page 234) summarizes the results. In all over fifty publicly available documents were used to construct the PSIOI. These documents were in the six categories that included marine operations, industrial properties, environment affairs, community outreach, news media coverage of the port, and the port commission. The first category of documents collected, coded, and analyzed related to 
marine operations. This process included reviewing documents correlated to the port's bulk, breakbulk, crude oil, project cargo, heavy lift, and ro-ro (roll-on-roll-off) commodity lines of business. Documents reviewed included those covering river-road routes, rail routes, trade routes, advanced supply chain issues, and port facilities.

The second category of documents related to industrial properties. This category included literature on currently available properties, properties that are not currently available, and various tenants and partners. This included a review of the port's master plan and associated or supporting documents. Documents were also collected, coded, and analyzed pertaining to the port's budget. These documents were especially helpful in providing data for coding since they signified a public commitment of resources to projects and programs. These commitments represented, in other words, the artifacts of institutional values.

The third category of documents related to the environment. State law requires the port to produce most, if not all, of these documents. The analytic procedure for these documents included a review of documents related to the port's stewardship efforts. This covered material related to wetland banks, habitat management, water quality, air quality, and the Washington State Environmental Policy Act (SEPA) process. Specific documents included active cleanup and completed cleanup notices along with bird-friendly building design information. Storm water and air quality permits, as well as documents related to other permitting processes were included in the coding effort.

The fourth category of documents related to community outreach. In many cases, the purpose of the documents was to reduce or eliminate information asymmetry between the port, the public, and the port's customers or clients. The port attempted to accomplish 
this by providing information to the public on the current and future status of port projects. The port published a rich portfolio of documentation related to the role of the port in the local, state, national, and global communities. These documents provided useful insight in the port's organizational culture.

Documents in the fourth category also were helpful in providing insight into the port's current and future intent. Documents collected and coded in this category included the community and annual reports meant for general consumption by the public. The port also commissioned studies on the economic impact of the ports marine, real estate, and overall operations that were especially useful. This coding effort also included coding of port partner documentation if it was publicly available. In many instances, these documents provided useful insight into the port's strategic environment.

The fifth category of documents related to news releases specifically intended for dissemination to the media. The target audience for these documents included citizens, customers, and clients. These documents comprised information provided to public media outlets related to the commission, community, commodities handled, economic studies, marine environmental impact, port-sponsored events, and industrial activities. This effort included collecting and coding information related to port partnerships, stewardship activities, leadership initiatives, and waterfront projects undertaken by the port. Also reviewed and coded was a port-generated media kit, various public affairs policies, and port-produced images.

The sixth and final category consisted of documents relating to the port commission. This included information regarding senior port leadership. This was a particularly rich source of information because it included the agenda and minutes of 
commission meetings. The agenda and minutes were a rich source of information because they reflected the priorities, concerns, and interests of the port as an organization and the public. Coding for the topics discussed and voted on in public meetings allowed for a high degree of fidelity in ascertaining the resilience, quality, accessibility, and efficiency of public services the commission intended to provide. This also provided unique insight into the goals and objectives of the port.

\section{Organizational Antecedents of Public Sector Innovation (OAPSI).}

This section presents an analysis of the data collected and coded during the field research phase of this study. The organization of this section is by theoretical domain. The order of the presentation of the domains and their associated antecedents is the same as their introduction in the previous chapter. Table 9 (see page 236) reflects the domains, antecedents, and the measure assigned to each observed independent variable. The following sections outline in detail the empirical observations and the resulting measurements. Table 9 reflects the OAPSI rating for the case study.

\section{Leadership-Client Co-Production.}

The first measure of adaptive interest alignment is the existence of feedback loops between the leader and their clients. There is significant evidence to support the proposition that robust feedback loops existed in the presence of innovation at the port. This resulted in a high measurement of this independent variable. In this context, it was possible to observe the presence of formal, informal, direct, and indirect feedback loops. One of the topical insights that emerged was the inherent complexity of feedback loops. 
Routinized and regularized processes characterize formal feedback loops. Therefore, for example, the twice-monthly commission meetings provided a formal structure for port leadership and their customers or clients to receive and provide feedback.

Ad hoc and serendipity characterizes informal feedback. This is the second emergent theme associated with the leadership-client co-production variable. Informally, the commissioners make themselves available by phone. Commissioners, the executive director, and other senior port leaders' attendance at public and private events represent other opportunities for informal feedback to occur. The lack of a principle (individual or organization) modulating the feedback loop characterizes direct feedback loops. This is the easiest mode of feedback to observe.

The existence of a principle that modulates the communication between other principles characterizes an indirect feedback loop. These loops established a series of dyadic relationships in which communication was iterative in nature. There is evidence to support the contention that indirect feedback loops existed in the presence of innovation at the port. In this regard, senior management created feedback loops with customers and clients. In these instances, the organization was the principle modulating feedback between leaders and their clients.

An example of the simultaneous nature of feedback loops was the leadership team's creation of a twice-weekly meeting. This gathering of the core cross-departmental team served to create and implement an innovative solution for the utilization of port property. The twice-weekly meeting cadence established for the team ensured near continuous real time feedback. Members of the team developed feedback loops between 
the organization and customers and clients. The leadership monitored these feedback loops and provided their own feedback to customers or clients thought this loop.

The second measure of adaptive interest alignment is leading with a vision.

Subjects routinely articulated innovation in the context of the ports strategic mission.

This resulted in a high measurement of this independent variable. One subject noted the "mission statement is a very valuable piece of how [the port] look[s] at these opportunities." The mission statement provided a structured and documented process for engaging in innovation. It had the added benefit of providing formal, tangible organizational support of innovation. As one subject noted, it embeds in the port's, "Can do' attitude." In this context, innovation is a normative value.

\section{Client-Organization Co-Production.}

The first measure of client-based prioritization is the existence of resource constraints. There was near universal agreement among the subjects regarding the presence of resource constraints. This resulted in a high measure assigned to this independent variable. This included financial, human, and/or informational resources. Like most public agencies, the port conducts an annual budget-setting process. One subject noted, however, that, "Not all budget information is known" at the time the budget is set. To address this situation, the port creates a reserve fund in the budget. It was through the subsequent emergence of innovation that port personnel "foresaw what was needed... budget-wise.”

An emergent theme is that innovation begets innovation. In this case, the innovation was shared lab space for equipment and processes that the biotech firms held 
in common. This is entirely new to biotech clustering. Traditionally private sector companies do not share a common pool of assets. The resources necessary to support this capacity were, however, beyond the resources of the port. The port looked into several options before petitioning the Governor's office for funds held in reserve for biotech initiatives. The port was successful in acquiring a commitment of funds from the state for implementing this additional innovation.

The data revealed the critical nature of information resources to the organization. One subject highlighted that the port "really needed to reach out and understand the biotech community, and [the port is] still doing that." This involved the "need to listen more than... talk." One emergent theme was that serendipity plays a rule in innovation. When asked to define serendipity in innovation a subject responded, "It means you are in places and open yourself up to innovation." Another subject explained the importance of marketing events in this regard. It is at these marketing events that the diffusion of innovation occurs. According to one subject, this is because "innovations are demonstrated at trade shows."

The second measure of client-based prioritization is co-production gap awareness. What this means in practice is that the participants engaging in co-production are conscious of a gap in resources. In other words, there was a recognition of the insufficient nature of the stock or supply of assets a leader or organization could draw upon. In the case of a public port, these can be economic, informational, social, or political in nature. All but one subject noted the existence of a co-production gap. Gaps included not enough funding to complete all required capital maintenance or not enough human resources to 
work on potential projects. The comments of the remaining subjects resulted in a high measure of this independent variable.

Another theme that emerged in the data is that the existence of an awareness of a co-production gap, as it relates to innovation, results in a negotiation with a customer or client. One attribute of co-production, moreover, is negotiation (Ostrom, Gardner and Walker 1994). One subject responded that there is "always innovation and always a negotiation" to highlight this linkage. Once identified, the gap serves to stimulate a discussion over "who was going to make what commitment financially," according to one subject.

One subject noted the "expectation of the customer is that [the port is] going to participate" from a resources perspective in innovation. Likewise, another subject observed that in this regard "[the port's] relationship with [its] customer, and how [the port is] going to get them into those spaces is, just that, it is a negotiation... [the port does] have some tools in [its] budget that [the port is] able to use." One subject stated that in this context, the sales team drives the working capital available for innovation. This indicates that the revenues from other business lines (i.e. industrial, marine, etc.) fund innovation at the port.

The port deliberately selects partners it perceives as willing negotiators since the port views the existence co-production gaps as resulting in negotiation. This theme echoed in the comment: "we always look for tenants that have similar values and principles, philosophies that the port does." This suggests that for success, special district negotiations must coalesce around the normative values held by each partner. The question emerges, moreover, over whose values matter. One subject provided the 
insightful comment that the port attempts to "match the community with the customer." In this regard, the three elected port commissioners serve to adjudicate the community's values.

The first measure of co-production readiness is the ability to sunset through coproduction. The model suggests that it is necessary to proactively sunset prior processes and resources for new processes and resources to emerge. This manifests, moreover, within the model as co-creation. The interview question related to sunsetting through coproduction asked: "did your organization examine and effectively phase out a current process, technology, or human resources in relation to a proposed innovation." The term "phase out" created a universally strong negative response by the subjects such that this antecedent received a low measurement.

The data suggests that the strategic environment is largely responsible for the sunset phenomenon. In this regard, the port had no control over the closure of the facility, which was largely the result of the economic downturn in 2008. This leads to the conclusion that the apparent role of the leader, the organization, and the customer or client is little more than attempting to mitigate the risk associated with an exogenous event. As an example, one subject noted that the port "worked with [the customer] on the best possible outcome to, for example, save jobs" once the facility closed. This is a risk mitigation strategy is explored in more detail below.

A theme that emerges is the role of path dependencies in public sector innovation. In this case, one of the catalysts for innovation was a significant disruption in a path dependent enterprise. The data from this question, therefore, supports the assertion that path dependencies are operational in public sector innovation. One possible conclusion is 
that the destruction of path dependencies opens the space for public sector innovation to occur. This would imply the presence of the phenomenon of punctuated equilibrium.

The contention that this innovation was more about phasing in something new rather than phasing out obsolescence is reflected in one subject's statement that "I wouldn't say that [the port] phased out anything for this opportunity... it was more of a phasing in of a new use." Another subject noted that about sunsetting "[the port] did not, the tenant did" in referring to the closure or sunsetting of the facility. The theme related to path dependency and phasing echoed in the response of another subject who shared the perspective that what the port was doing was the "phasing in of a commercial space." Another subject suggested that the correct perspective is that "Terminal 1 is [a] brand new business line."

The second measure of co-production readiness is information symmetry. In other words, whether the parties involved in co-production share information equally. This does not mean, however, that all participants possess the same information. In this regard, the data supports the contention that the port valued open communication and sought information symmetry among its customers and clients. The evidence supported assigning a high value to this independent variable. There is one important caveat to this contention. The port sought to keep certain commercial information private. The intent is to protect the port's - and by extension the communities - financial interests. There are, however, strict controls that limit the confidentiality of port information.

Another theme that emerged is the close connection between information symmetry at decision points and the phasing of projects or programs in (or out) of existence. One subject pointed, for example, to the link between decision points and the 
port's master plan as a potential source of information symmetry. The port's master plan is foundational to the multi-dimensional communication of the port's intent to pursue opportunities in targeted commercial or business sectors. By outlining what the port plans to develop, the master plan provides the vision and guidance on the nature of any expected innovation. Developing the master plan is only the first step. The next step is to complete a concept development plan. These public documents serve as tools to engender information symmetry.

\section{Organization-Leader Co-Production.}

The first measure of the antecedent of organizational structure and culture is transformational leadership. The data indicated strong support for the contentions that transformational leadership empowered port employees to engage in innovation. Therefore, for example, one subject observed, "leadership dictates the level of innovation." In this regard, a subject noted, “[the port's] leadership basically outlines the goals and objectives, what is needed, and then trusts individuals to accomplish those goals" through innovation. Empowerment was demonstrated thorough the comment that "people were given the resources they needed to move ahead on their goals."

A theme that emerged from the data was that a culture of trust is necessary to foster the co-production of innovation (Ingram, Schneider, and Deleon 2007). In other words, individual members of the organization had a firm belief in the truth, ability, and reliability of organizational leaders. Trust in the context of this case meant that senior leadership provided the resources for success and trust that junior members of the organization are good public stewards. Senior leaders were successful in creating an 
organizational culture that engendering the reciprocation of trust horizontally and vertically in the organization.

Comments like "[individuals] were given the latitude to go out and do it" support the value of establishing an atmosphere of trust. This is especially crucial in an environment built around operational execution for customers or clients. Therefore, for example, several subjects noted that senior port leadership reinforced trust at the department head level of the organization. In this instance, it involved the suggestion that all leaders should "know that people in [their] division will make a decision and know that it will ultimately be right."

The leadership team specifically designated a champion for innovation at the port. This subject stated, "My job is innovation, I only have one employee but I have 100." Even with top management support, this is not an easy task. For example, the champion admitted to feeling "stymied by the need to process the change that is offered." This was in reference to the process of change management around innovation at the port. Overall, the subject articulated that it is altogether natural and healthy for the organization to evaluate organizational innovation in a critical manner.

One subject noted the presence of "leadership at all levels of the organization." The subject was reference to project management activities undertaken by the port. Another theme that emerged was the desire by senior leadership to create a sense of community at the port. This sense of community is evident in the use of cross discipline teams to address a wide range of issues at the port and create a sense of common ownership in creating solutions. The perception existed that senior leaders wished to "build a sense of community with a focus on the long term... having faith [the employee] 
will be part of it." The data, therefore, supports assigning a high value to the presence of transformational leadership as it relates to innovation in the public sector.

The second independent variable of organizational structure and culture is the ability to experiment. The port took a very cautious approach to experimentation and, consequently there was a low measure to this independent variable. While a subject noted that the "leadership and organization [of the port] is open to experimentation and new ways of doing things if they are better." It was clear from subsequent comments that the time, cost, and risk of experimentation was something to avoid in practice. Another subject noted that in relation to the bio-medical innovation that the "experiment happened on paper before it ever moves forward."

The subjects also noted the existence of experimentation in a wide range of contexts. To illustrate the point a subject noted that the port may, "experiment with recruiting new customers, trying to get the right fit... sometimes it works and sometimes it does not." Referring to the bio-medical innovation a subject noted that the "whole project is like a pilot- - $[\mathrm{a}]$ new and emerging business and industry.” The port went through a recent reorganization and in the context of experimentation; a subject characterized this activity as an "organizational design experiment."

The measure of the antecedent of organizational incentives is inducements for improvement activities. The question related to this antecedent probed for the presence of both intrinsic and extrinsic incentives related to improvement or innovation. In this context, intrinsic rewards include inspirational motivation, individual autonomy, and intellectual stimulation. Extrinsic rewards, on the other hand, appear as contingent financial rewards. The data indicated the port primarily employs intrinsic incentives and 
there was very little evidence to support the contention the port relies on extrinsic rewards. To highlight this point one subject simply responded "completely the first [option], intrinsic." This independent variable received a medium measure given the presence of intrinsic rewards and the absence of extrinsic rewards.

One theme that emerged was the value of creating a "culture of sharing credit." One subject, nevertheless, noted the challenge in recognizing this organizational value: the port “tries to celebrate success as an organization, but [it doesn't] do well at it sometimes." This suggests another emergent theme is operative such that a gap exists between the intended and the actual level of intrinsic rewards offered by management. One of the modalities the port employed to institutionalize this value is to embed the value in organizational rituals. One example was through port leadership "recognizing specific contributions at staff meetings." This has the effect of routinizing this cultural value as it relates to intrinsic rewards.

One subject cited a recent paid time off (PTO) benefit enhancement. This had the advantage of providing an intrinsic incentive whereby "people respond to freedom of time and being able to schedule other life events around the work they absolutely have to get done." In the context of a public agency, one subject observed, "financial reward must look like merit." This raises, but does not answer, the question of what rewards for individual merit look like in relation to innovation. One theme to emerge is that organizational culture plays a vital role in providing incentives for innovation. In this regard, one subject noted that the "basic structure of the organization creates expectations." These expectations serve to define what and how the organization rewards individual efforts. 
The measure of the antecedent of risk-taking preferences is individual risk-taking preference. The data served to highlight the multifaceted nature of risk. Overall, the data revealed a surprisingly low level of risk tolerance. One subject stated, "You have to have a little risk in every portfolio... [individuals] look at risk, but figure out a way to mitigate the risk." However, the same subject noted also that the "organization does not promote risk, but is not turned away by it." This is the case one subject suggests because "risktaking is constrained by the public governance aspect."

Several themes emerged regarding risk and public sector innovation. The first is that risk is ubiquitous and, as such, requires careful mitigation. This included both the frequency and severity of negative outcomes. One subject noted that "[the port does] take risks in how it is develop[s] alternatives for current customers and future customers." Another subject stated, "If you want to stay in business you need to take prudent risks." Mitigation also takes a number of forms, including a strategy to "adopt what [individuals] are familiar with as [they] approach a new market."

Another emergent theme is that risk and its mitigation takes a number of forms. For example, risk can have an organizational or process basis. This is reflected in the comment by a subject that at the port: "[Individuals] balance the risk with the reward and... don't necessary take a lot of risk in altering policies all the time because [the port] what [s] to have some consistency in how it is [the port] arrive[s] at decisions, and how [they] work together as a group." Another theme is the temporal nature of risk perception. In this context, the port takes a long-term perspective reflected in the comment that the port is, "Not looking at short term, [the port] [is] in it for the long term." 
Another theme to emerge is that circumstances and conditions in the strategic environment condition risk preferences. In other words, risk mitigation has a strategic component. On subject noted that the port was "not entirely risk adverse, it depends on the size" of the risk. The port recently embarked on an energy project that was a costly investment. To the extent this investment will pay off, it is expected to do so years into the future. While none of the subjects mentioned the energy project in the context of risk management, it was clear from the interviews that this project influenced perceptions around risk.

This had the effect of shifting the port from "embracing risk to reigning it in." Therefore, "[the port's] policy at this time is to be risk adverse." The measurement that emerges from the data is that innovation occurred despite low levels of individual and organizational risk-taking preferences. In some areas, the port chose deliberately to avoid risk. The area of organizational values is illustrative with one subject noting that "[the port] don't take a risk in the values [it] bring[s] to decision making and the innovation that stems from that."

\section{$\underline{\text { Within-Case Analysis. }}$}

Table 8 contains the results of the coding of the public sector innovation organizational index (PSIOI) for the first case. The assignment of measurements to the independent variables occurred during the data-coding phase of this research project. This table depicts the subsequently summarization of the results. This summation occurred at the conclusion of the PSIOI data collection phase and preceded the data collection, coding, and analysis phases for the OAPSI rating. The PSIOI represents an expected 
value of the dependent variable and is the result of summing the measures of the attributes of the independent variables.

Table 8. Case 1 Public Sector Innovation Organizational Index (PSIOI)

\begin{tabular}{|c|c|c|c|c|}
\hline \multirow{2}{*}{ Independent Variable } & \multicolumn{4}{|c|}{ Attributes: High (+)/ Med (0)/ Low (-) Measure } \\
\hline & Resilience & Quality & Accessibility & Efficiency \\
\hline 1.Existence of feedback loops & + & 0 & + & + \\
\hline 2. Lead with a vision & + & + & + & + \\
\hline $\begin{array}{l}\text { 3. Existence of resource } \\
\text { constraints }\end{array}$ & + & 0 & + & + \\
\hline 4. Co-production gap awareness & + & + & 0 & + \\
\hline 5. Sunset through co-production & + & 0 & + & + \\
\hline 6. Information symmetry & + & + & + & 0 \\
\hline 7. Transformational leadership & - & + & + & + \\
\hline 8. Ability to experiment & - & - & - & - \\
\hline 9. Incentives for improvement & 0 & - & - & - \\
\hline $\begin{array}{l}\text { 10. Individual risk-taking } \\
\text { preference }\end{array}$ & - & - & - & - \\
\hline Individual Summary Rating & +1 & 0 & +1 & +1 \\
\hline & & Overall & nmary Rating & +1 \\
\hline
\end{tabular}

Construction of the index involved identifying positive instances of resilience, quality, accessibility, and efficiency for each independent variable. An independent variable received a high measure $(+)$ with three or more net positive observations. An independent variable received a medium measure (0) with one or two net positive observations. No net positive observations or a net negative observation indicated a low measure (-). A negative observation is one in which the empirical observation was in the 
opposite direction of resilience, quality, accessibility, and efficiency. If the observed measure was high, a value of $(+)$ was assigned. A medium measure received a (0). If the measure was low, a value of (-) was assigned.

The construction of individual summary ratings for instances of resilience, quality, accessibility, and efficiency involved combining the unweighted values of the measures associated with each of the ten independent variables for that attribute. In other words, the unweighted summary of the measures of the instances of the attributes assigned to each independent variable is the basis for the overall individual summary rating of the attributes. The next chapter discusses some of the potential implications inherent in this approach. The overall PSIOI summary rating resulted from an unweighted average of the four individual summary ratings. The result is a measure of the expected value of the dependent variable of public sector innovation.

Table 9 contains a summary of the measurements of the organizational antecedent of public sector innovation (OAPSI) rating. The measures assigned to the independent variables is discussed in the earlier section of this case related to the OAPSI. The OAPSI represents the actual value of the dependent variable reflecting the degree of the coproduction of innovation in the public sector. This rating reflects a summary of the coding conducted during the OAPSI rating phase of this study. A detailed description of the methodology for constructing this rating is contained in the previous chapter. If the measure was high, a value of $(+)$ was assigned. A medium measure received a ( 0$)$. If the measure was low, a value of (-) was assigned.

The OAPSI rating is a simple additive and unweighted index. The mathematical effect of this methodology is to a create disparity such that the ten independent variables 
are weighted differently. Therefore, for example, measures of the independent variable related to adaptive interest alignment have a disparately large impact on the overall score. This is because in that domain only two independent variables exist, as opposed to four independent variables in client-organization and organizational-leader co-production domains. This approach was deliberate and serves to dampen mathematically the effect of potential outlier variables. The next chapter discusses the implications of this conservative approach.

Table 9. Case 1 Organizational Antecedents of Public Sector Innovation Rating

\begin{tabular}{|c|c|c|c|c|}
\hline \multirow[t]{2}{*}{ Domain } & \multirow[t]{2}{*}{ Antecedent } & \multirow[t]{2}{*}{ Independent Variable } & \multicolumn{2}{|c|}{ Measure } \\
\hline & & & $\begin{array}{l}\text { High }(+) / \\
\text { Low (-) }\end{array}$ & $\begin{array}{l}\text { Rating } \\
(1 / 0)\end{array}$ \\
\hline \multirow{2}{*}{$\begin{array}{l}\text { Leader-Client } \\
\text { Co-Production }\end{array}$} & \multirow{2}{*}{$\begin{array}{l}\text { Adaptive Interest } \\
\text { Alignment }\end{array}$} & Existence of feedback loops & + & \multirow[t]{2}{*}{+1} \\
\hline & & Lead with a vision & + & \\
\hline \multirow{4}{*}{$\begin{array}{l}\text { Client- } \\
\text { Organization } \\
\text { Co-Production }\end{array}$} & \multirow{2}{*}{$\begin{array}{l}\text { Client-based } \\
\text { Prioritization }\end{array}$} & Existence of resource constraints & + & \multirow[t]{4}{*}{+1} \\
\hline & & Co-production gap awareness & + & \\
\hline & \multirow[t]{2}{*}{$\begin{array}{l}\text { Co-Production } \\
\text { Readiness }\end{array}$} & Sunset through co-production & - & \\
\hline & & Information symmetry & + & \\
\hline \multirow{4}{*}{$\begin{array}{l}\text { Organizational- } \\
\text { Leader Co- } \\
\text { Production }\end{array}$} & \multirow{2}{*}{$\begin{array}{l}\text { Organizational } \\
\text { Structure/Culture }\end{array}$} & Transformational leadership & + & \multirow[t]{4}{*}{0} \\
\hline & & Ability to experiment & - & \\
\hline & $\begin{array}{l}\text { Organizational } \\
\text { Incentives }\end{array}$ & $\begin{array}{l}\text { Incentives for improvement } \\
\text { activity }\end{array}$ & 0 & \\
\hline & $\begin{array}{l}\text { Risk-taking } \\
\text { Preferences }\end{array}$ & Individual risk-taking preference & - & \\
\hline \multicolumn{4}{|c|}{ Overall Summary Rating } & +1 \\
\hline
\end{tabular}

The overall values of the OAPSI and PSIOI rating are both equal to positive one (1.0) in this case. The model suggests that with an OAPSI approaching positive one (1.0), 
it is possible to expect co-produced innovation to occur. This is the case since the OAPSI rating is a measure of expected innovation. The model posits, furthermore, that in cases where the PSIOI approaches positive one (1.0), the conditions for innovation actually occur. The PSIOI rating is a measure of actual innovation. Since the indices depict the expected and actual values of the dependent and independent variables, they serve to support the validation of the model.

In summary, the results of this case served to validate the theoretical framework this dissertation put at risk. The model predicted that the presence of certain antecedents would create the pathway to the co-production of public sector innovation. The dependent variable of co-produced public sector innovation emerged in the presence of these antecedents. It was possible to measure the presence of the antecedents thorough the empirical observation of certain independent variables. The empirical evidence, therefore, supported the contention that the theoretical framework presents a valid understanding of the phenomenon. The model also provides a valid prediction of the conditions that result in the co-production of public sector innovation.

\section{Case 2: Creating Intergovernmental Capacity}

The second case involves a port engaged in the creation of capacity in other local general and special-purpose governments. In other words, the focus is on capacitybuilding external to the organization. This case involves a medium-sized public port located along a major navigable waterway in the Pacific Northwest. The special district holds a diverse portfolio of business lines. This includes maritime, aviation, industrial, 
recreational, and real estate holdings. The port's district is unique among the cases in that it includes a mix of both rural and suburban communities.

The suburban zone of the port district is adjacent to a major metropolitan area. It serves as a bedroom community for this area. The maritime holdings include deep-water facilities capable of handling ocean-going vessels and a marina housing recreational and residential watercraft. The primary cargos handled at this port are various bulk commodities that arrive by rail or truck. A well-developed state road network and national Cass 1 rail lines serve the port's facilities. The port also has easy connections to the interstate highway system.

\section{Background and Setting.}

In this case, a five-member publicly elected commission adopted a policy that created an intergovernmental partnership aligned with the port's mission. The commission provided broad general direction necessary for port staff to achieve specific goals and objectives related to creating local governmental capacity perceived as necessary for economic development. An executive director, reporting directly to the commission, provided day-to-day leadership of a small staff tasked with creating and executing port policy. State law in which this port resides grants public ports general taxing authority. State law also limits the port's activities to those involving economic development. One subject estimated that approximately ninety-five percent of the citizens of the three counties that this port district spans reside within the geographic boundaries of the port district. 
In this case, the port maintains a general tax representing less than $1 \%$ of its overall revenue. It retains a strong financial position through its various commercial enterprises. It also has a solid commercial position relative to its size. This allowed it to explore innovative ways to pursue its economic development mission. From a public policy perspective, economic development reflects efforts that seek to improve the economic well-being and quality of life of a community through job creation, income growth, and an expanding tax base (Kraft and Furlong 2013). As such, transportation systems, public safety, and community affairs play a role in economic development. With this insight in mind, the commission, executive director, and port staff sought community partnerships aligned with the port's economic development mission. The result was the intergovernmental partnership program (IPP) designed to award funding to qualifying projects. This innovation provided general-purpose and special district governments the opportunity to enhance their services to local communities. The intent of the IPP was to target projects that advanced economic development within the port district. In keeping with the port's mandate, this program was only available to organizations that operated within the port's district boundaries.

The port received and reviewed applications for this program. It subsequently made several awards to city, county, and local utilities based on an agreed upon set of criteria. The ports senior leaders, in consultation with the commission, established the criteria for the program. Projects selected for funding were one-time capital investments and did not represent an ongoing annual financial commitment. When forming a partnership, the port executed an intergovernmental agreement committing a designated 
amount of funds to a specific project. In exchange, the partnering public agency provided matching funds for the project.

\section{$\underline{\text { Public Sector Innovation Organizational Index (PSIOI). }}$}

Table 10 (see page 257) summarizes the public sector innovation organizational index for this case. Like the first case, constructing the PSIOI involved collecting documents in the categories of marine operations, industrial property, environment activities, community outreach, media coverage of the port, and the port commission. In the first case, it was possible to collect a relatively balanced portfolio of documents across all six categories for coding and analysis. There was considerable skewing, however, of the document portfolio collected and coded for this case.

This skewing was in the direction of the community outreach, news releases, and port commission categories. Less material was publicly available in the other categories. This is not an issue since the documents in the other categories provided sufficient for constructing the PSIOI. The separation of the documents into six categories was to support efficiency of the collection effort and not for a methodological reason. The effect, therefore, of the skewing on the outcome was negligible. In all, it was possible to collect, code, and analyze over forty documents in the six categories.

The remainder of this section briefly outlines the documents in each of the six categories used to construct the PSIOI. The first category of documents collected, coded, and analyzed were related to marine operations. Advisory committee meeting minutes related to the port's marina operations were the primary documents collected, coded, and analyzed in this category of documents. These documents proved useful in understanding 
how the port interacts with its customers and clients since they document many of these exchanges. The documents served to indicate the manner in which the port institutionalized the exchange of information to address issues of information symmetry.

The second category of documents related to industrial property. Advisory committee meeting minutes and the comprehensive master plan were among the documents used to compose the PSIOI. The port relied upon advisory committees to engage with customers and clients in key business sectors. The advisory committee structure served to institutionalize communication processes the port believed necessary to achieve information asymmetry. The committee structure also served to provide transparency of the ports actions and intentions to the public.

The third category of documents related to the environment. There were very few publicly available documents in this category. There was, therefore, very little to directly collect, code, or analyze in this category. This is not an issue since the documents in the other categories provided sufficient for constructing the PSIOI. In a sense, these categories are artificial constructs to assist in the document search. They are not central to the coding or analysis of the data collected. In many instances, documents in other categories contained references to environmental issues. This was especially the case in the media coverage and port commission documents.

The fourth category of documents related to community outreach. Documents in this category included the port's strategic business plans, newsletters, and various reports. These documents primarily related to the port's efforts to address the issue of information asymmetry. Financial reports and requests for proposal (RFP) were also among the documents collected, coded, and analyzed. In this regard, financial documents reflect the 
actual commitment of public resources. If public policy is what is what the public funds, then these documents provide tangible insight into the organizational implementation of public policy.

The fifth category of documents related to the news media. This involved collecting, coding, and analyzing media coverage from three competing local newspapers. These media organizations were organic to the communities in the port district's jurisdiction. Each news outlet was active in the community. They primarily covered local events and issues of community interest. The same media correspondents from each newspaper tended to cover and report on port district related activities. This continuity in coverage served to provide context to the news reporting.

The sixth and final category of documents related to the port commission. Minutes from commission meetings and public resolutions provided a rich source of documentation. In addition to board minutes, collection and coding involved commission resolutions and budgetary documents. These documents were rich in detail and context, although, the latter resulted in some challenges. This was the case since references in the budget documents were not always easy to discern. In some instances, the commission documents were incomplete or contained conjecture.

\section{Organizational Antecedents of Public Sector Innovation (OAPSI).}

This section presents an analysis of the data collected and coded during the field research phase of the second case study. As in the previous case study, the organization of this section is by theoretical domain. Table 11 (see page 258) reflects the domains, antecedents, and the measure assigned to each empirically observed independent variable 
in this case. The table reflects the OAPSI rating assigned to this case study. This reflects the actual measure of the dependent variable of co-produced public sector innovation that is present in this case. The section that follows contains a discussion of the empirical observations and the resulting measurements of the independent variables that resulted in the OAPSI rating.

\section{Leadership-Client Co-Production.}

In general, there exists within the political economy of our society the normative value that resource extraction by the state requires the consent of the affected citizens. Without this consent, the theory goes; the actions of the state are not legitimate. In other words, no taxation without representation. The converse of this corollary, no representation without taxation, animates this case study. An emergent theme in this case is that interests adapt and align around the communities' normative values.

Taxation allows for the normative values of the community to find voice in the way citizens pursue economic development. While Morgan et al. (2013) might suggest that the benefits of public ports generally accrue to parochial interests, some of the costs to operate the port are borne by the public though a local port tax. The innovation that is the subject of this case involved the manner in which the port used public funds to achieve its larger organizational vision. The innovation was to create capacity for economic development in their peer local governments.

Adaptive interest alignment is the antecedent of leader-client co-production. The first measure of adaptive interest alignment is the existence of feedback loops between the leader and their customers and clients. The latter includes citizens of the port district. 
In this case, there was evidence of strong feedback loops on both the formal and informal level. This lead to a high measure of this independent variable. Routinized communications regarding innovation processes and projects included regular meetings and correspondences. So, for example, there is ample evidence in this case that port leadership received continually feedback from the general public regarding their appetite for port taxes and their opinion of the port's use of those funds to support public sector innovation.

Normative values, including the desire to "give back to the community," animated the interest in innovating, according to one subject. Port leadership perceived their primary social and organizational mission as "economic development." In this context, the primary metric is the "creation and sustainment of jobs," commented a senior port leader. The means for achieving economic development, moreover, are wide ranging. Top leadership at the port articulated, for example, that public safety, community affairs, and transportation play a part in economic development. One subject commented that this expansive view of economic development enabled the port to "think out of the box" when considering innovation.

The second measure of adaptive interest alignment is leading with a vision. Port leadership specifically articulated innovation in its published vision statement. The port also identified innovation as a port principle. Documentation obtained during the field research phase of this study revealed that the port's vision refers to the desired future state of the institution. In this case, the port articulated their vision: "To be a leading, innovative port focused on economic opportunities which benefit... future generations." 
Documents provided during subject interviews also listed innovation as one of the port's principles.

Several of the subjects related innovation to creativity as normative principles that drove their actions at the port. This was especially the case in the context of the specific innovation under study. Several documents reviewed during the data collection phase also confirmed this linkage. Individuals occupying senior leadership positions referenced the same or similar source documents during the interview phase. Subjects in leadership or supervisory roles either were participants in the creation of the guidance or were involved through consultation as to the content of the guidance during its creation and/or revision. Port documents co-listed innovation and creativity. It is important to note, however, that the port gave the former primacy of place within the principle. There was, therefore, a clear distinction between creativity and innovation. The theme emerged, therefore, that there is a close link between creativity and innovation. The nature of this linkage requires further investigation.

The interview subjects routinely and consistently articulated the linkages between their actions and the guidance provided by senior port leaders. Each member of the port staff interviewed, for example, could recall and clearly articulate innovation in relation to the institutions vision and principles. This was the case without prompting or probing in every interview. Several subjects specifically indicated that innovation was the basis for their actions in the public sphere. In this case, there is a direct linkage between the port's institutional vision and innovation.

One interview subject noted that the port collectively referred "to the vision, mission, and port principles as their guidance." There is no mention of innovation in the 
port's mission statement. Only during the last iteration of the guidance, moreover, did the port list innovation as a principle. This recent shift emphasis serves to indicate the emerging perception that innovation is an increasingly important component of public governance. This lead to a high measure of the independent variable of leading with a vision.

\section{Client-Organization Co-Production.}

The antecedents of client-based prioritization and co-production readiness animate the domain of client-organization co-production. The first measure of clientbased prioritization is the existence of resource constraints. The measure of the existence of resources constraints was medium in this case. This port district contains both urban and rural communities. This is salient since, while the urban communities have largely experienced an economic recovery, the rural communities continue to lag behind in economic terms. This drove the need for special districts to be sensitive to the existence and effect of the socio-economic divisions on the communities in which they reside. This was an emergent theme.

The existence of resource constraints was particularly acute in the rural parts of the port district where the theme emerged that, "Ports are often chasing their own tails." This comment suggests that the port was continually pursuing the promise of new economic opportunities, but often lacked the necessary infrastructure to attract customers. In other words, the comment reflects a condition whereby the port was unaware that the lack of economic infrastructure is a result of an absence of forward thinking investment by the port. Therefore, for example, one subject acknowledged the presence of resource 
limitations by noting "the budget reality of what [the port]" has resulted in "missed opportunities because of the lack of facilities." The port was, in a sense, ignorant of an emergent requirement in sufficient time to make an investment to develop the necessary infrastructure to secure customers.

Elements in the strategic environment activated by the port's participation in the energy sector fostered the perception that limitations related to available public safety infrastructure existed. In this context, resource constraints drove the desire to innovate in order to guarantee mitigation of perceived risk posed by a particular port tenant. Participation in the energy sector resulted in a significant windfall to the port. It also served to inspire innovation across the spectrum of port activities to address concerns that arose due to this participation.

There is evidence that customer and client demand drove funding priorities at this port. The port's budget committee provides an illustration. The budget committee represents an institutional mechanism for ensuring that the demands of customers or clients receive budget priority. The port appoints five citizens to the budget committee, which serve alongside the five elected commissioners. The appointment of each member is at-large from the community and occurs through a consensus process. The budget committee is responsible for drafting the port's annual budget. While port staff provide the initial recommendation, and the commission has final approval, the appointed members provided an additional access point for the community.

Co-production gap awareness is the second measure of client-based prioritization. There is evidence the port actively co-produced operational budgets that embraced needed public sector innovation with customers and clients. In this case, the observed 
measurement of this independent variable was high. It is important to note the coproduction of the port's operations budget followed the identification of the innovation. The port's operations budget reflected known expenses and forecasted future costs. In this case, the port allocated specific funding through the budget process to support the goal of building capacity in local governmental organizations. The budgeted funds had the effect of indirectly supporting economic development though the creation of governmental capacity in other institutions.

An awareness of a co-production gap existed at two levels within this case. The first was at the municipal or other special district level and the second was at the port level. Once a municipal agency or special district became aware of a co-production gap, it scanned the environment to identify opportunities to close the gap. In this case, the organization identified the IPP as an appropriate resource for closing the gap. At the same time, the port could sense the existence of a co-production gap in the community in which it imbedded.

The multidimensional awareness of the existence of co-production gaps is evident in the comment that the community "got [into] a big conversation as to what is needed" during the IPP award process. It is notable that the port specifically sought out other examples of community investment by other special districts and general-purpose governments. This involved a concerted effort at "benchmarking partnership opportunities," according to one subject. Senior leadership expressed the opinion that the result of this benchmarking was not encouraging. Another subject noted that the port was discouraged by another special district from adopting such a program. There was a 
perception that this type of program creates friction within the community. This friction was a result of various stakeholders vying for limited communal resources.

The port and the partnering agency created an intergovernmental agreement (IGA) to implement the IPP. The IGA served as the mechanism to formalize and institutionalize the process necessary to "leverage funds to accomplish projects benefiting citizens living within the port district." Agreement to participate in an IGA was a prerequisite for the municipal agencies and special districts seeking a partnership with the port. The IGA process served to document the existence of a co-production gap at the agency.

The second antecedent of client-organization co-production is co-production readiness. The first measure of co-production readiness is the ability to sunset through coproduction. In this case, the observed measure of ability to sunset through co-production was low. There was no evidence the port of examining or phasing out any current processes, technology, or human resources in relation to the innovation. Indeed, one of the subjects noted that the port "did not change much about how [it] [did] business" when addressing the issue of sunsetting through co-production. In the words of another subject the "new program was not intended to supplant anything."

The economy in the county where this port district resides faired "as bad if not worse" than the rest of the state during the recent downturn. A significant decline in traditional resource extraction industries hit the rural areas hard as well. All this left the county population economically vulnerable, according to the same subject. The subject implied that this put the port under pressure to perform, since the community viewed it as the "biggest job creator and economic engine for the county." In this role, the port 
engaged in path dependent behavior by attempting to maintain their current tenant base. This specifically involved avoiding the sunset of existing projects or programs. In this regard, the theme that path dependencies play an important role in innovation emerged.

There was a clear indication that the port contemplated a phase-out situation for the IPP when considering an award in particular and other port projects generally, although this was not the desired end state. Port leadership considered this program specifically, and other projects generally, as additions to the port's economic development portfolio. In consideration of a possible sunset, the port was only willing to consider shovel-ready capital projects for the IPP. The port was averse to considering multiyear, annuity type projects involving personal costs. There was a perception that an annualized commitment—for instance paying for additional headcount in a partner agency — would serve as a barrier to future sunsetting.

The second measure of co-production readiness is information symmetry. In this regard, an analysis of the data suggests the port routinized information sharing. The observed measure of the independent variable of information symmetry was high in this case. In this context, the evidence indicates that the port viewed community engagement and education as critical to their mission. One subject stated, "you can never say enough" to individuals in the community when discussing the benefits of port programs to the citizens of the county. Another subject noted that the port routinely sought out the "opportunity to help educate the public." This included placing a priority in communicating with internal and external customers.

In addressing the issue of information symmetry, one subject stated that, "[individuals] try as much as [they] can, [the port is] a little organization, and [the port] 
do[es] a lot of outreach for our size." In this context, the port did not have a functional specialist engaged in public relations or community engagement. The expectation was that each staff member was a practicing generalist in his or her specific area of responsibility. There was also evidence of resources dedicated to information sharing specific to this innovation. Therefore, for example, the port took steps to ensure the publication of the results of the IPP awards in the local newspapers and that the port newsletter highlighted the benefits of the IPP to the community. In addition, the same subject stated that stakeholders received a letter from the port touting the IPP award.

Senior port leadership also sought to inform employees of port initiatives. The perception of port management was that this was a particularly effective means of communicating the port's message to the larger community. This was the case, since port employees interacted closely with members of the local communities in which they worked and lived. There was a belief that this was especially true in the ports rural communities where a tightly knit social fabric ensured that even relatively minor investments in public infrastructure were widely noticed. Community outreach and educating the public also occurred directly and indirectly through, for example, encouraging port employee participation in local social and cultural organizations. The intent of these actions was to foster information symmetry.

\section{Organizational-Leader Co-Production}

Organizational incentives, risk-taking preferences, and organizational structure and culture represent the antecedents that animate the organizational-leader coproduction domain. The presence of transformational leadership traits and the ability to 
experiment serve to measure organizational structure and culture. The first measure of the antecedent of organizational structure and culture is transformational leadership. In this case, it was possible to identify three or more characteristics of transformational leadership. The organization, for example, shared a collective organizational culture, displayed adaptability, and responded proactively to their environment. The observed measure of the independent variable of transformational leadership was high.

The evidence suggests that senior leadership shared and understood the collective organizational consciousness. They used this understanding to provide a clear idea of their desired action. Senior leadership disseminated guidance from through various formal and informal mechanisms. The guidance articulated by senior leadership had the effect of promoting a cohesive response to organizational and business opportunities. A senior leader stated, for example, that the "governing board gave [him/her] a great deal of autotomy." A subject on the staff commented that management "gave [her/him] a great deal of latitude to make this work." These comments were specific to the creation and implementation of the IPP.

There was evidence that the port continually adapted to changing market conditions and opportunities in a dynamic manner. This included a receptivity to new ideas. There was also an understanding that success was dependent on the effort of the entire team. In this context, one subject reported, "[port employees] really work as a team here." Another commented that, "silos don't work here." Recently, the port institutionalized the process of providing individual coaching and mentoring for employees through a formal review process. This enabled senior leadership to stay attuned to the staff's feelings and the collective consciousness of the organization. In this 
regard, port leadership sought out opportunities to inspire, energize, and intellectually stimulate port staff.

The second measure of organizational structure and culture is the ability to experiment. There was evidence of non-systemic or random experimentation. This occurred against a backdrop of senior leadership providing "a lot of leeway in how [the port] structure[s] a project" according to one subject. The observed measure of this independent variable was medium in this case. Experimentation was, furthermore, opportunistic in nature and tied to the organizations core mission. Along these lines, one subject commented that innovation is "encouraged, but not a lot of opportunities" exist to engage in the practice. This was the case according to the subject because, "[individuals] have to respond to the market..." and, "[the port has a] certain process [the port] must adhere to by state law."

Despite these limitations, one subject commented that the port is "always open to new opportunities." In this regard, the port sought to "promote and push initiative," according to a subject. Despite this willingness, the public nature of the organization tempered the appetite to experiment. In this context, one subject stated that the organization had a "willingness to experiment as long as it falls within the lines of public trust." This highlighted another theme, the perception that the normative values of maintaining the public trust and ensuring financial stewardship could come into conflict with the practice of innovation.

Non-systemic or random experimentation was an artifact of organizational size and resource constraints. This reflected in the emergent theme that the size of the organization and the resources available to that organization either support or impede 
experimentation. This was specifically the case with this organization's human resources. In this context, there were not specific resources dedicated to generating innovation. The organizational expectation was that staff would pursue innovation at their own discretion. One subject stated in reference to the IPP that, "[the port] did not have a formal 'this [is] going to be a pilot' but [the port] did have an informal be ready to pull back." One subject, when considering the IPP, stated that the mindset was that the "first time lets test drive it... throttles back" to us an aviation euphemism to typify the port's approach to of experimentation.

The measure of the antecedent of organizational incentives is incentives for improvement and innovation. In this case, the observed measure of this independent variable was medium. A robust measure of incentives would include both intrinsic and extrinsic rewards. There were clear indications that the port's senior management understood the role incentives play is motivating the staff. For example, a senior leader noted the port is "always trying to make sure people are being rewarded for working hard." This was also evident in a recent review of the port's recognition program. The result was several enhancements to the program.

There was, however, little evidence of the port employing intrinsic rewards tied directly to improvement or innovation. This is in large measure a result of the nature of the port's public sector model of compensation. This system consisted of a base salary with annual merit based adjustments. There was also a non-discretionary component tied to the financial performance of the organization. As such, intrinsic rewards for innovation were at best indirect. It was, therefore, possible to see an increase in compensation 
through a merit-based adjustment because of innovation, but only if innovation created tangible results.

Evidence of extrinsic rewards was more robust. As one subject observed about extrinsic incentives "it is not the amount; it's how you do it." In this case, port leadership included public recognition, encouragement, and time off. The objective of these rewards was to empower individuals to pursue innovation. The extrinsic reward system at the port included, for example, small tokens of recognition like coins with the port logo embossed on them. Individuals engaging in behavior and activities that senior management wished to encourage or reward received these coins. This reflects the value the port placed on extrinsic rewards.

The measure of the antecedent of risk-taking preferences is individual risk-taking. The measurement of this independent variable was low in this case. The subjects demonstrated a wide range and depth of understanding regarding the role of risk and its effect. The consensus was that risk embeds in the strategic environment. One subject, for example, asserted that, "risk is used to create something." One theme that emerged is that the organizations perception of risk is multifaceted. This included personal (or safety) risk, environmental risk, commodities handled risk, legal risk, budget risk, and compliance risk. In this regard, risk is a contextual phenomenon. Each of these facets of risk, moreover, requires a different framework for analysis and understanding (Morgan 2006).

The port staff placed a high value on risk mitigation. In the context of this case, risk management required a balanced approach. There was a perception from one subject's that taking risk was legitimate to the extent that "larger goals are met." Risk, 
therefore, required active management by port staff. This involved the port staff deliberately engaging in the identification and assessment of the effects of uncertainty on the ports objectives. This implies taking steps to minimize, monitor, and control the impact of unfavorable events to maximize organizational opportunities for successful outcomes. In other words, risk management involved minimizing uncertainty in the pursuit of organizational goals. Risks, therefore, involved an implied promise of significant reward. Technological, human, and organizational resources played a role in risk management as well (Morgan 2006).

The primacy of place afforded the protection of public property from perceived misuse or misappropriation by port district staff emerged during the course of this case study. While there was an expressed willingness to avoid taking risks, a theme emerged that risk tolerance varied over time. Noting its temporal nature, one subject commented that recently the port had become risk adverse after being "burned by large projects." In this context, organizational memory around past failures or successes served to modulate risk-taking preferences.

\section{Within-Case Analysis.}

Table 10 contains a summary of the coding of the public sector innovation organization index (PSIOI) for the second case. As in the previous case, the assignment of measures to the independent variables that comprise the index occurred during the data-coding phase of the PSIOI's creation. As in the previous case, this preceded the data collection, coding, and analysis phases of the OAPSI creation. As previously mentioned, the PSIOI represents an expected value of the dependent variable. Creating the PSIOI 
involved the generation of individual summary ratings for each independent variable across the resilience, quality, accessibility, and efficiency attributional categories.

Table 10. Case 2 Public Sector Innovation Organizational Index (PSIOI)

\begin{tabular}{|l|c|c|c|c|}
\hline \multicolumn{1}{|c|}{ Independent Variable } & \multicolumn{4}{c|}{ Attribute: High (+)/ Med (0)/ Low (-) Measure } \\
\cline { 2 - 5 } & & Quality & Accessibility & Efficiency \\
\hline 1. Existence of feedback loops & + & + & + & + \\
\hline 2. Lead with a vision & + & + & + & + \\
\hline $\begin{array}{l}\text { 3. Existence of resource } \\
\text { constraints }\end{array}$ & + & + & 0 & 0 \\
\hline 4. Co-production gap awareness & + & + & - & - \\
\hline 5. Sunset through co-production & - & $\mathbf{0}$ & - & - \\
\hline 6. Information symmetry & + & + & + & 0 \\
\hline 7. Transformational leadership & + & + & + & + \\
\hline 8. Ability to experiment & - & - & - & - \\
\hline 9. Incentives for improvement & $\mathbf{0}$ & - & $\mathbf{0}$ & $\mathbf{0}$ \\
\hline $\begin{array}{l}\text { 10. Individual risk-taking } \\
\text { preference }\end{array}$ & $\mathbf{0}$ & $\mathbf{0}$ & $\mathbf{0}$ & $\mathbf{0}$ \\
\hline \multicolumn{1}{|l|}{ Individual Summary Rating } & +1 & +1 & +1 & $\mathbf{0}$ \\
\hline \multicolumn{2}{|l|}{ Overall Summary Rating } & +1 \\
\hline
\end{tabular}

The individual summary ratings reflect the arithmetic sum of the values of each of the measures of the independent variables associated with each of the four attributes. In this case, when a net of three positive observations occurred in each category coding stopped. Three such observations resulted in a high measurement of the independent variable. If the observed measure was high a value of $(+)$ was assigned. An independent variable received a medium measure with a net of one or two positive observations. A 
medium measure received a (0). No net positive observations or a net negative observation indicated a low measure. If the measure was low, a value of (-) was assigned. An explanation of the rational for these thresholds is in the previous chapter.

Table 11 contains a summary of the measurements of the organizational antecedents of public sector innovation (OAPSI) outlined in the section pertaining to the second case. This reflects a summary of the coding conducted during the data analysis phase. If the measure was high, a value of (+) was assigned. A medium measure received a (0). If the measure was low, a value of (-) was assigned. The OAPSI rating represents an actual value of the dependent variable. The next chapter discusses some of the implications to the model that flow from this data. The concluding chapter also presents some potential implications inherent in this approach to calculating the PSIOI and OAPSI rating.

An unweighted average calculation of the measures within their respective domain resulted in a measure of that domain. The overall OAPSI value resulted from an unweighted average calculation of the three domains. The overall values of PSIOI and OAPSI rating are both equal to positive one $(+1.0)$ in this case. The model suggests that with a PSIOI rating approaches positive $(+1.0)$ there is an expectation that innovation is occurring. PSIOI is a presumed measure of expected innovation. The model posits, furthermore, that in cases where the OAPSI approaches positive one $(+1.0)$ the conditions for innovation occur. OAPSI is a presumed measure of actual innovation. The indices are a useful tool for depicting both the expected and actual manifestation of the coproduction of organizational innovation. 
Table 11. Case 2 Organizational Antecedents of Public Sector Innovation Rating

\begin{tabular}{|c|c|c|c|c|}
\hline \multirow[t]{2}{*}{ Domain } & \multirow[t]{2}{*}{ Antecedent } & \multirow[t]{2}{*}{ Independent Variable } & \multicolumn{2}{|c|}{ Measure } \\
\hline & & & $\begin{array}{l}\text { High }(+) / \\
\text { Low (-) }\end{array}$ & $\begin{array}{l}\text { Rating } \\
(1 / 0)\end{array}$ \\
\hline \multirow{2}{*}{$\begin{array}{l}\text { Leader-Client } \\
\text { Co-Production }\end{array}$} & \multirow{2}{*}{$\begin{array}{l}\text { Adaptive Interest } \\
\text { Alignment }\end{array}$} & Existence of feedback loops & + & \multirow[t]{2}{*}{+1} \\
\hline & & Lead with a vision & + & \\
\hline \multirow{4}{*}{$\begin{array}{l}\text { Client- } \\
\text { Organization } \\
\text { Co-Production }\end{array}$} & \multirow{2}{*}{$\begin{array}{l}\text { Client-based } \\
\text { Prioritization }\end{array}$} & Existence of resource constraints & 0 & \multirow[t]{4}{*}{+1} \\
\hline & & Co-production gap awareness & + & \\
\hline & \multirow{2}{*}{$\begin{array}{l}\text { Co-Production } \\
\text { Readiness }\end{array}$} & Sunset through co-production & - & \\
\hline & & Information symmetry & + & \\
\hline \multirow{4}{*}{$\begin{array}{l}\text { Organizational- } \\
\text { Leader Co- } \\
\text { Production }\end{array}$} & \multirow{2}{*}{$\begin{array}{l}\text { Organizational } \\
\text { Structure/Culture }\end{array}$} & Transformational leadership & + & \multirow[t]{4}{*}{0} \\
\hline & & Ability to experiment & 0 & \\
\hline & $\begin{array}{l}\text { Organizational } \\
\text { Incentives }\end{array}$ & $\begin{array}{l}\text { Incentives for improvement } \\
\text { activity }\end{array}$ & 0 & \\
\hline & $\begin{array}{l}\text { Risk-taking } \\
\text { Preferences }\end{array}$ & Individual risk-taking preference & - & \\
\hline \multicolumn{4}{|c|}{ Overall Summary Rating } & +1 \\
\hline
\end{tabular}

In summary, the result of this case study served to validate the model. In this case, there was an alignment of the expected value of co-produced public sector innovation with the actual observation of this phenomenon. This case demonstrated that it is possible to observe the dependent variable in the presence of the antecedents. It was possible to observe the presence of the antecedents through the observation of certain independent variables. The empirical evidence, therefore, supports the contention that the theoretical framework put at risk is valid. The model provides a valid understanding of the social interactions that lead to the phenomenon. The model also provides a valid prediction of when the co-production of public sector innovation will emerge. 


\section{Case 3: Reconciling Conflicting Demands among Customers and Clients}

The Pacific Northwest contains a mix of special district ports that operate in urban and rural communities. The first case involved a port district in a major urban area. The setting for the second case was a port district that spanned urban, suburban, and rural communities. The third case involves a port district in a rural community along the Pacific Ocean. In the previous two cases, the port's customers and clients were dependent on navigable inland waterways for access to their facilities. In this case, the port is contiguous to the Pacific Ocean. Agriculture, natural resource extraction, recreation, and tourism are the major economic drivers within this port district.

The special district that is the subject of this case has facilities that support commercial fishing, oceanographic research, and public recreation. The port is in the process of making the capital improvements necessary to reopen an international commercial shipping terminal. This terminal will export natural resources, including locally harvested timber. This case deals with the ports innovative approach to reconciling conflicting local demands on their existing infrastructure. In this instance, the demands stemmed from two natural resource extraction industries - one land-based and the other reliant upon the ocean.

\section{Background and Setting.}

In this case, a five-member, publicly elected board oversees the port and sets policy. The port's general manager and staff then execute that policy. Similar to the ports in the previous cases, this special district has maritime, recreational, and industrial 
holdings. However, unlike the other two ports that are the subject of this dissertation, this special district does not have good connections to the North American surface (rail and truck) transportation network. This port instead has a facility that supports commercial fishing operations. Many of the individuals engaged in commercial fishing make the port district their home.

The infrastructure that supports the commercial fishing industry at the port is aging and inadequate for current industrial requirements. In some instances, for example, the facilities are fifty to sixty years old. Since the port built these facilities, there has been an increase in the size of commercial fishing vessels. There has also been an increase in the number of commercial fishing vessels homeported at the port. Many of the largest vessels homeported at the port belong to the distant ocean fleet. These vessels travel, for example, to Alaskan waters to fish. The port is also home to a large number of smaller vessels engaged in coastal fishing. Changes to the size and number of commercial fishing vessels has impaired the port's ability to service the fleet. Simply put, the current infrastructure is inadequate to meet the needs of the commercial fishing fleet.

The geographic area of the port's jurisdiction is rich in timber resources. The port was re-developing and promoting an idle international shipping terminal to support the export of logs. This terminal has been inactive for well over a decade due to a decline in the extraction of natural resources due to regional political and economic forces. The port was hoping to capitalize on recent economic developments in the timber industry to reopen the facility designed to support the export of logs to the Asian market. Having outgrown the commercial fishing facilities, the fleet has begun using the idle international 
shipping terminal. At the time of this case study, the distant water commercial fishing fleet is the primary user of the international shipping terminal.

In this case, an empowered employee took the initiative to develop an innovative plan for addressing capacity at the commercial fishing facility. Commercial shipping and fishing interests were in competition for limited space along the port's waterfront. The result was social friction and conflicting demands on limited public port infrastructure. The port managed to moderate these conflicting interests up to a point. This case study involves an innovative approach a port employee took on his or her own initiative, and with the encouragement of top management, to moderate these conflicting economic interests.

$\underline{\text { Public Sector Innovation Organizational Index (PSIOI). }}$

Table 12 (see page 277) contains a summary of the public sector innovation organization index (PSIOI). Like the previous cases, this index was the result of coding documents in six categories. This section contains an overview of the documents collected, coded, and analyzed to construct the PSIOI for this case. The goal of this section is to provide context to the process of creating the PSIOI. Similar to the previous cases, this section will serve as a roadmap to analyzing the methodological approach undertaken in this dissertation. The intent is to provide the information necessary for an independent assessment of this research project.

Construction of the PSIOI involved collecting, coding, and analyzing approximately 130 unique documents. There was a more or less balanced number of documents in each of the six categories. This is a significant increase in the number of 
documents compared to the previous two cases. The reason for this increase is that there were more publicly available documents from this organization. The documents were, however, of a similar or lower quality than what was previously collected to create the PSIOI in the earlier cases. High-quality documents reflect those that were rich in both context and content. It is the quality, and not the quantity, of the documents that is paramount in constructing the PSIOI. In this regard, the overall quality of the quantity of the documents examined was generally consistent between cases.

The first category of documents collected, coded, and analyzed related to marine operations. This included cost-benefit narratives, grant applications, and requests for qualifications related to the port's maritime operations. The latter included documents related to proposed maritime projects. The second category of documents related to industrial property. This primarily involved collecting information on tenant leases. Documents in both of these categories served to provide perspective on the core economic benefits of the port to the community. Many of the documents in these first two categories were useful in documenting the port's political economy.

The third category of documents related to the environment. Documents in this category, for example, included those required by the port for local, state, and national permitting processes. Specifically, this included documentation related to activities like dredging navigation channels and port berths. Environmental documents included those records related to construction projects on port property. In some instances, the approval of indigenous tribes was required for projects to proceed. In these instances, there was a public documentation of the approval. 
The fourth category of documents related to community outreach. Port newsletters, grant narratives, community updates on port projects, and letters of support from stakeholders were included in this category. The documents collected also included meeting minutes from port committees, economic impact analyses, and the ports formal facility maintenance and operations plans. The community outreach documents contained informational material on the social and economic contributions the port makes to the local community.

The fifth category of documents related to public media. This included portproduced content for public broadcasting. Public media products included material tailored to the print, radio, television, and other electronic media outlets in the port district and beyond. News releases by the port and media information kits provided by the port were the primary documents collected, coded, and analyzed in this category. These public documents largely reflected attempts by the port and their employees to address issues of perceived information asymmetry between the port as an organization and the local community. The goal was to achieve information symmetry between port leadership, the port as an organization, the ports customers and clients, and citizens residing in the port district.

The sixth and final category of documents related to the port commission. These documents were a rich source of information. This category had the largest number of documents. It included documents relating to special commission meetings, regular commission meetings, and joint working session meetings. Biographic documents were included in this category as well. This category also included documents relating to 
budget committee meetings and commission workshops. These documents were useful in contextualizing the port's strategic environment.

\section{Organizational Antecedents of Public Sector Innovation (OAPSI).}

This section presents an analysis of the data collected and coded during the field research phase of this case study. As in the previous cases, the organization of this section is by domain. Table 13 (see page 279) reflects the domains, antecedents, and the measurements assigned to each observed independent variable that results in the OAPSI rating. The table reflects the OAPSI rating assigned to this case study. This section contains a detailed discussion of the observations and the resulting measurements of the independent variables.

\section{Leadership-Client Co-Production}

The first measure of adaptive interest alignment is the existence of feedback loops between leader and their clients. There is significant evidence to support the proposition that a robust network of feedback loops existed between port leadership and the port's customers and clients. This included evidence of routinized communications regarding the bottom-up innovation process observed in this case. These feedback loops took the form of regularly scheduled meetings, written reports, and written or electronic correspondences. This suggests that the measure of this independent variable is high.

When discussing the creation of feedback loops, one subject noted that in their role as a senior leader that this was something they do. The size of the port in this case is relatively small. It imbeds within a stable polity. As a result, there is a high level of 
organizational intimacy. It is possible, therefore, for individual members of the organization to develop robust interpersonal relations with individuals across the organization and within the community. The result is a rich network of formal and informal feedback loops.

The port recently experienced a change in their top leadership. The previous team had been in place for almost two decades. The new leaders embarked on an effort to formalize internal processes and procedures at the port. As a result, the port identified several gaps. One subject noted that closing these gaps resulted in "internal resistance." To overcome this challenge, senior management created formal and informal feedback loops within and between the organization and the organizations leadership. The objective of creating these feedback loops was to produce transparency and trust within the organization necessary to create the space for organizational leadership to flourish. Senior leaders believed this was necessary to create the conditions for change and innovation at the port.

One theme that emerged was the use of feedback loops to amplify the demand signal for co-producing innovation. In this case, the commercial fishing community was instrumental in spurring innovative thinking around expanding local capacity. This resulted in a strong network of feedback loops between the port, the ports leaders, and their customers and clients. This is especially apparent when dealing with conflicts over infrastructure at the port. In this regard, the proliferation in the size and number of vessels in the fishing fleet created a critical demand for infrastructure at the port. This, in turn, created conflict with other user groups. 
The port's leadership acknowledged this user group conflict and suggested the need for a robust network of formal and informal feedback loops to address the conflict. For example, one subject noted that in dealing with "issues between fishermen, sometimes you have to go down and smooth things over... [and] threaten to kick them out if they don't straighten up." In addition to this informal, interpersonal approach to dealing with customers and clients, the port established a commercial fishing users group to formalize communications. The port sponsored this committee. It included individual owners and operators of vessels in the fishing fleet that used port facilities. This group met on a regular basis with port leadership to discuss issues of mutual interest.

The second measure of adaptive interest alignment is leading with a vision. In this case, the port had a formal vision, mission statement, and governing values. Individual employees were able to recognize the vision. The employees were also able to articulate the importance of the vision, mission, and governing values in relation to innovations necessary to meet the demands of the ports customers and clients. This suggests a measurement of this independent variable is high in this case.

Subjects could consistently articulate innovation in relation to the institution's vision, mission statement, and governing values. The vision statement specifically calls out the commercial fishing fleet as a customer or client of the port. The mission and vision statements did not explicitly mention innovation; however, several of the subjects considered it implicate in how they would accomplish the port's mission. One subject said, "[the port] review[s] mission, values, and goals every year," in noting the perpetual nature of this document. 


\section{Client-Organization Co-Production}

The first measure of client-based prioritization is the existence of resource constraints. One subject noted the challenge of "not having enough [infrastructure] ... [and] the infrastructure is very old." Another subject noted the challenge of "trying to find enough space" for commercial fishing vessels at the port. In referring to the vessels of the commercial fishing fleet, one subject noted, "there are more coming..." to the port and "they are bigger." This led to the fishing fleet using of facilities designated for general-purpose cargo vessels. A conflict subsequently arose when the port faced the prospect of "getting log ships back so [break bulk vessels] will displace the Alaskan fleet."

The evidence suggests that the organization's budget priorities reflected specific demands of customers or clients for innovation. In this case, the port leadership was clearly aware to the need to co-produce solutions with the commercial fishing community. There is also evidence the port made investments in infrastructure that supported the commercial fishing fleet. Commercial shipping represented a significant economic opportunity for the port. Taking advantage of the commercial shipping revenue opportunities was a stated priority for the leadership team at the port. All this suggests a high measurement of this independent variable.

This innovation resulted from a mid-level leader seeing an increasing gap in the port's service offering. Once identified, the individual initiated the co-production of innovation with the port's customers and clients. This individual was in a positon to do this based on their organizational role and their unique relationship with the ports customers and clients. In this regard, the leader was seeking to "throw out a conceptual 
idea... this might be the next big thing [the port] [is] going to shoot for." With regard to this specific innovation; however, there was no budget for it at this time. This leader believed a necessary first step in implementing innovation was to create the momentum for innovation through the process of co-creation.

The second measure of client-based prioritization is co-production gap awareness. One subject noted an awareness of the co-production gap in stating that it is "really reflected more on the commercial fishing docks... [the port] just [has] so many needs." To address this, the same subject stated the "commission created a commercial user group... a committee whereby the harbormaster brings forth his priories for the budget year and shares that with the users who help refine that list which then gets forwarded to all the department heads and [management] kind of prioritize the entire port."

In this case, there is robust evidence the organization actively co-produced operation budgets that embraced needed public service innovation with customers and clients. This suggests a high measurement of this independent variable related to this case. This measurement is evident in the establishment of the commercial fishing user group. The role of this committee was to assist port leadership in prioritizing capital projects. It also supported the creation of operational budgets. This reflects the emergent theme identified in an earlier case that the existence of a co-production awareness gap results in negotiation with customers and clients.

Senior leadership indicated the commercial fishing user group was an institutional mechanism that had the benefit of providing a "better sense [the port] [is] meeting [the] needs of the client." This institutional mechanism powerfully conditioned the way individuals regarded and behaved towards each other. Per the same subject, it also serves 
to "build a better relationship." In this regard, it served to reconcile competing resource demands. The commission also asserted their belief that more local engagement was necessary such that the port "Needed more boots on the ground and not shiny shoes."

The first measure of co-production readiness is the ability to sunset through coproduction. There was some evidence of efforts to examine and phase out current processes, technologies, or human resources in relation to innovation at the port. There was also some evidence the port engaged in organization innovation to build organizational capacity for innovation. The management team intentionally attempted to phase out certain organizational activities they believed added little value to the customers or clients of the port. The various initiatives the port has undertaken recently suggest a medium measurement of this independent variable.

Recent internal organizational changes illustrate the port's efforts to create the space for organizational innovation. One subject noted that, "[the port] did eliminate a position and set up a different organization flow." The result was to eliminate perceived bureaucratic barriers to innovation in two key areas. The first was to upgrade the role of senior leaders, which involved a shift in organizational responsibilities. Accountability for initiating and managing key organizational projects and initiatives shifted to department heads. Previously these organizational responsibilities were a staff function. The effect of transferring this task into a line function was to grow organizational capacity for innovation within the port.

The second effort was to apply a metrics-based approach to knowing the organization and identifying organizational gaps. An example of this is the installation of a computerized maintenance management system (CMMS). The design of this system 
allowed the organization to address perceived gaps in the organization's understanding of the state of the port's infrastructure preservation efforts. This tool also had the effect of facilitating the addition of useful life to the port's capital infrastructure. These actions resulted in an organizational realignment necessary to meet the long-term needs of the port's customers and clients. This resulted in the opening of a permissive space for innovation within the organization.

With regard to the specific innovation under study, one subject noted that the port was not phasing out but adding capabilities to support the commercial fishing fleet. The same subject subsequently noted, "all the [commercial fishing] infrastructure there is so old it must be phased out anyway." In other words, this innovation had the potential of transforming the ports infrastructure related to commercial fishing. This transformation would change the way the commercial fishing fleet operated in the Pacific Northwest.

The second measure of co-production readiness is information symmetry. In this regard, there was evidence of routinized information sharing and dedicated resources to information sharing. This included a priority on communicating with internal and external customers. These communications served to share with customers, clients, and the community the benefits of innovation. Information symmetry involved efforts by port leadership to articulate their value proposition to the community. This suggests a high measurement of this independent variable.

One of the challenges facing senor port leadership is, according to one subject, "making sure the lenses line up... what's good for the community... and... what's good for the port's [fiscal] solvency." One subject noted the value of commercial shipping at the ports international terminal. While the port recognizes the value proposition in having 
a diversified portfolio of customers and clients, it also recognizes that potential conflicts may emerge over limited port resources. In addition to the commercial fishing users group, one subject noted, "[the port] [is] creating a terminal users group so when the commercial shipping comes [the port] [has] a plan for addressing issues since there is a finite amount of property."

The leader championing the innovation identified in this case pursued both vertical and horizontal information symmetry. The champion sought to vet the innovation within formal organizational channels. For example, the champion noted, "my boss tells me "this looks great, keep doing it." The champion also sought horizontal information symmetry using the institutional mechanism of the commercial fishing committee. As an example, when referring to the commercial fishing user group the champion responded, "they really seem to like [this innovation]."

\section{Organizational-Leader Co-Production.}

The first measure of the antecedent of organizational structure and culture is transformational leadership. In this case, it was possible to identify three or more characteristics of transformational leadership. The organization, for example, pursued the identification of needed changes and responded proactively to their strategic environment. They also displayed the ability to form the necessary strategic partnerships to bring their vision to fruition. This resulted in a high measure of the independent variable of transformational leadership.

Regarding the identification of needed changes, one subject noted that port leadership sought to "transfer more of the decision making down... close to the ground... 
and make the people closest to the work make many of the decisions." This had the effect of accelerating innovation and change within the organization. One senior leader noted in characterizing their role that they were "hired more for introspection and [focus on] personnel development." In this respect, the port was transitioning from a top-down to a collaborative structure. Coincidentally, the port "replaced seven of the eight management positions..." and began the process of "taking a more progressive approach to management and leadership."

One senior leader noted that the port's ability to respond proactively to their strategic environment started with their own position. Foundational to this leader's ability to transform the port is a supportive board that knows its role as policy makers. This led the same leader to comment, "I see my role as providing resources and leadership for people that technically know their jobs." This is helpful since one subject, when discussing senior leadership, shared the perception that "[the port has] a fairly new administration for the most part and none of them really know what the needs are." This has the paradoxical effect of allowing leaders at all levels to emerge in the organization.

Critical to the implementation of innovation at the port is the ability to form the necessary partnerships to bring the port's vision to fruition. The formation of a formal committee structure served to bring the stakeholders together and provided them a voice in co-producing public sector innovation. In this regard, one leader responded that, “[he/she] want[ed] people to share their ideas." Another subject noted during the transition to the transformational leadership paradigm "[the port] [is] doing a lot of things organizationally that the public might not think is so sexy, and they don't see as much." In this regard, a subject noted that the port is seeking to answer the question "how do you 
get more by providing resources." These comments reflect an ongoing shift towards a transformational leadership style at the port.

The second measure of organizational structure and culture is the ability to experiment. Experimentation involves the systemic adoption of new ideas, methods, or activities on a limited or one time basis to understand their impact on the organization or institution (Dubin 1969). There was minimal evidence of experimentation observed while assembling this case study. This suggests a low measure of the independent variable related to the ability to experiment. In referring to experimentation, one senior leader stated this is not an area that leadership is contemplating devoting any resources.

The measure of the antecedent of organizational incentives is the presence of both intrinsic and extrinsic incentives for improvement activities. While there was little evidence of extrinsic rewards at the port, there was significant evidence of intrinsic incentives. These took the form of personal and pubic recognition. This suggests a medium measure of the independent variable related to the existence of incentives for improvement activity. In several instances, there was a direct link between intrinsic rewards and innovation. There was also a normative aspect to this port's desire to innovate.

Concerning extrinsic rewards, one subject said, "There are not a whole lot of financial rewards for working here." It is a strong association with the community that motivated this subject. It is worth noting that this subject is a lifelong resident of this special district. This subject has also spent time working in the commercial fishing industry before this individual joining the port. The subject placed a great deal of value on the need to maintain the health and viability of the commercial fishing fleet. 
Therefore, the subject equated the commercial fishing fleet to a proxy for the economic and social health of the community. The subject articulated this notion in the context of a public good stating, "[the port's] carrot is keeping the fishermen here."

The port clearly focuses on providing intrinsic rewards. This manifests in efforts to improve the work experience for port employees. One subject noted that the port had a culture that retained individuals longer other employers in the region. Improving the work environment included providing opportunities to upgrade personal and professional skills. This is reflected in a senior leader's indication that the port would "love to have [port employees] here forever; but [the port] want[s] to give [port employees] the tools that may help them go somewhere else."

In focusing on the desire to provide intrinsic rewards around personal and professional development, one subject noted the ports recent focus on an "annual goal setting and... training program" as evidence of this mindset. Another subject noted the ports recent interest in "allowing facility managers to take more ownership" as an expression of the desire to provide more leadership opportunities at the port. Intrinsic rewards also took the form of empowering employees, including involving them in the decision-making process. Therefore, for example, senior leader stated, "by me meeting with each employee once a month, [employees] feel they have a more direct line to [senior leadership]."

The measure of the antecedent of risk-taking preferences is individual risk-taking preferences. Several subjects indicated that there was an express desire to avoid risk at the port. There were also indications of extensive risk mitigation activities tied to innovation. This suggests a low measure of the independent variable related to individual 
risk-taking preferences. In referring to risk and risk-taking, one senior leader noted, "I would say the risk-taking is more in the feedback loop." In this context, the same senior leader noted that, "my style is that I don't want 'yes' people."

In other words, there is a bias towards achieving honest consensus before acting. The evidence suggested that this group consensus and seeing an issue from a diversity of viewpoints served as a risk mitigation strategy. Similar to the previous cases, there is a nuanced and multifaceted view of risk expressed by the subjects. There was an understanding of the varied nature of risk and a stated desire to mitigate its undesirable consequences. It was referenced in several different modalities include organizational, personal, health, and safety.

\section{Within-Case Analysis.}

Table 12 contains a summary of the coding for the public sector innovation organization index (PSIOI) for the third case. As in the previous cases, there was an assignment of a measurement to an independent variable associated with the index during the data-coding phase of the PSIOI. Like the previous cases, the development of the PSIOI preceded the data collection, coding, and analysis phase of the OAPSI. If the observed measure was high a value of (+) was assigned. A medium measure received a (0). If the measure was low, a value of (-) was assigned. As previously mentioned, the PSIOI represents an expected value of the dependent variable.

As in the previous cases, the individual summary ratings for the attributes of resilience, quality, accessibility, and efficiency were the result of an unweighted summary of the values associated with each attribute of the independent variables. In this 
case, coding stopped and a measurement of the independent variable as high (+) was recorded when three net positive observations were noted. This is consistent with the previous case. An independent variable received a medium (0) measure with one or two net positive observations. No net positive observations or net negative observations indicated a low measure (-). The next chapter discusses some of the potential implications inherent in this approach.

Table 12. Case 3 Public Sector Innovation Organizational Index (PSIOI)

\begin{tabular}{|l|c|c|c|c|}
\hline \multirow{2}{*}{ Independent Variable } & \multicolumn{3}{|c|}{ Attribute: High (+)/ Med (0)/ Low (-) Measure } \\
\cline { 2 - 5 } & Resilience & Quality & Accessibility & Efficiency \\
\hline \hline 1. Existence of feedback loops & + & + & + & + \\
\hline 2. Lead with a vision & + & + & + & 0 \\
\hline $\begin{array}{l}\text { 3. Existence of resource } \\
\text { constraints }\end{array}$ & + & + & + & $\mathbf{0}$ \\
\hline $\begin{array}{l}\text { 4. Co-production gap awareness } \\
\text { 5. Sunset through co-production }\end{array}$ & + & + & + & + \\
\hline $\begin{array}{l}\text { 6. Information symmetry } \\
\text { 7. Transformational leadership }\end{array}$ & + & $\mathbf{0}$ & $\mathbf{0}$ & $\mathbf{0}$ \\
\hline 8. Ability to experiment & $\mathbf{0}$ & $\mathbf{0}$ & $\mathbf{0}$ & $\mathbf{0}$ \\
\hline 9. Incentives for improvement & $\mathbf{0}$ & $\mathbf{0}$ & + & $\mathbf{0}$ \\
\hline $\begin{array}{l}\text { 10. Individual risk-taking } \\
\text { preference }\end{array}$ & $\mathbf{0}$ & - & - & - \\
\hline Individual Summary Rating (1/0) & $+\mathbf{+ 1}$ & $+\mathbf{1}$ & $+\mathbf{+ 1}$ & $\mathbf{0}$ \\
\hline & \multicolumn{2}{|c|}{ Overall Summary Rating (1/0) } & $+\mathbf{1}$ \\
\hline
\end{tabular}

Table 13 contains a summary of the measurements of the organizational antecedents of public sector innovation (OAPSI) for the third case. The previous section 
discussed in detail of assignment of these values. The OAPSI rating reflects a summary of the coding and analysis conducted during this case study. The methodology followed for constructing the OAPSI rating is contained in previous chapters of this dissertation. If the measure was high, a value of $(+)$ was assigned. A medium measure received a (0). If the measure was low, a value of (-) was assigned. As previously mentioned, the OAPSI rating represents an actual value of the dependent variable.

Table 13. Case 3 Organizational Antecedents of Public Sector Innovation Rating

\begin{tabular}{|c|c|c|c|c|}
\hline \multirow[t]{2}{*}{ Domain } & \multirow[t]{2}{*}{ Antecedent } & \multirow[t]{2}{*}{ Independent Variable } & \multicolumn{2}{|c|}{ Measure } \\
\hline & & & $\begin{array}{c}\text { Yes }(+) / \\
\text { No (-) }\end{array}$ & $1 / 0$ \\
\hline \multirow[t]{2}{*}{$\begin{array}{l}\text { Leader-Client } \\
\text { Co-Production }\end{array}$} & \multirow[t]{2}{*}{$\begin{array}{l}\text { Adaptive Interest } \\
\text { Alignment }\end{array}$} & $\begin{array}{l}\text { Existence of feedback } \\
\text { loops }\end{array}$ & + & \multirow[t]{2}{*}{+1} \\
\hline & & Lead with a vision & + & \\
\hline \multirow{4}{*}{$\begin{array}{l}\text { Client- } \\
\text { Organization } \\
\text { Co-Production }\end{array}$} & \multirow[t]{2}{*}{$\begin{array}{l}\text { Client-based } \\
\text { Prioritization }\end{array}$} & $\begin{array}{l}\text { Existence of resource } \\
\text { constraints }\end{array}$ & + & \multirow[t]{4}{*}{+1} \\
\hline & & $\begin{array}{l}\text { Co-production gap } \\
\text { awareness }\end{array}$ & + & \\
\hline & \multirow[t]{2}{*}{$\begin{array}{l}\text { Co-Production } \\
\text { Readiness }\end{array}$} & $\begin{array}{l}\text { Sunset through co- } \\
\text { production }\end{array}$ & 0 & \\
\hline & & Information symmetry & + & \\
\hline \multirow{4}{*}{$\begin{array}{l}\text { Organizational- } \\
\text { Leader Co- } \\
\text { Production }\end{array}$} & \multirow[t]{2}{*}{$\begin{array}{l}\text { Organizational } \\
\text { Structure/Culture }\end{array}$} & $\begin{array}{l}\text { Transformational } \\
\text { leadership }\end{array}$ & + & \multirow[t]{4}{*}{0} \\
\hline & & Ability to experiment & - & \\
\hline & $\begin{array}{l}\text { Organizational } \\
\text { Incentives }\end{array}$ & $\begin{array}{l}\text { Incentives for } \\
\text { improvement activity }\end{array}$ & 0 & \\
\hline & $\begin{array}{l}\text { Risk-taking } \\
\text { Preferences }\end{array}$ & $\begin{array}{l}\text { Individual risk-taking } \\
\text { preference }\end{array}$ & - & \\
\hline \multicolumn{4}{|c|}{ Overall Summary Rating } & +1 \\
\hline
\end{tabular}

An unweighted average calculation of the measures within their respective domain resulted in a summary value for that domain. The overall OAPSI value resulted from an unweighted average of the three domains. The overall values of PSIOI and the 275 
OAPSI rating are both equal to positive one $(+1.0)$ in this case. The model posits, furthermore, that in cases where the PSIOI approaches positive one $(+1.0)$ there is an expectation that the conditions for innovation will occur. PSIOI is a measure of expected innovation. The model also suggests that as the OAPSI rating approaches positive one $(+1.0)$, innovation is occurring. The OAPSI rating is a measure of actual innovation. In this case, it was possible to observe the co-production of public sector innovation.

In conclusion, the result of this case study was to validate the model. In this case, the expected value of co-produced public sector innovation aligned with the actual observation of this phenomenon. The observation of the dependent variable occurred in the presence of the antecedents. The observation of certain independent variables confirmed the presence of the antecedents. The empirical evidence supports the contention that the theoretical framework put at risk is valid. The model provides a valid understanding of the social interactions that lead to the phenomenon. The model also provides a valid prediction of the emergence of the co-production of public sector innovation.

\section{Between-Case or Cross-Case Analysis}

The case study technique is a robust scientifically based method for examining complex phenomenon like the co-production of public sector innovation (Yin 1994). The previous sections of this chapter objectively presented three individual case studies in sequence. There was a deliberate bias towards selecting cases in which there was an anticipation of observing a manifestation of the phenomenon. In each case, it was possible to empirically observe and measure independent variables that indicate the 
existence of the phenomenon. Each case represented an instance in which it was possible to observe the causal links the model suggests. The case studies also explain how and why the co-production of public sector innovation occurs.

The previous sections of this chapter presented a within-case analysis of three instances in which the phenomenon under study manifest. Each case involved an individual exploration of the phenomenon within a context that was independent of any other instances of the phenomenon. While the study of an individual case is a powerful tool it, nevertheless, represents a singular observation of an occurrence of a complex phenomenon (Yin 1994). The benefit of a singular observation is that it is possible to draw inferences and make conclusions regarding the model. The danger of a singular observation is that it could also represent a particularistic manifestation of the phenomenon. It is possible to address this weakness by increasing the number of observations (Yin 1994). This will provide additional evidence to support the model.

It is now time to change gears and focus on the three cases from the cross-case perspective. Up to this point, the examination of each case occurred in isolation from the others. The individual approach used in the earlier sections of this chapter was vertical in orientation. In these sections of the chapter, there was no reference to other cases. In other words, the terms of reference were only in relation to the model. The remainder of this chapter uses a horizontal orientation to examine the cases. This is akin to lining up the three cases and examining them through a single proverbial sight line.

The remainder of this chapter presents the results of the cross-case analysis. In this analysis, the terms of reference are the other cases. The objective of this approach is to achieve a synthesized understanding of the model. In other words, instances of 
covariance serve to indicate the existence of correlation between similar independent variables resident in the model. This analytical approach also strives to identify spurious correlation and extraneous variables. This will allow for the generation of the findings, conclusions, and recommendations presented in the next chapter. Cross-case analysis represents a powerful tool for empirically validating the theoretical model.

Cross-case analysis using three or more cases serves to strengthen the findings related to the model (Yin 1994). In this dissertation, the PSIOI and OAPSI rating are a measure of the dependent variable. Individuals engaged in the co-production of public sector innovation are the dependent variable. The PSIOI and OAPSI rating results from a measure of ten independent variables. In the context of this phenomenon, cross-case analysis makes a powerful and persuasive argument for the efficacy of the model. What follows is a validation of the model through the simultaneous and holistic analysis of the three cases. This synthesis has the advantage of providing a robust examination of this phenomenon. Yin (2009) suggests this leads to a more complete understanding of social phenomenon.

This section presents a holistic, sequential examination of the three domains of the model. The design of the study minimized any cross-scale and cross-level dynamics between cases by providing a singular, uniform measure of a common variable across all three cases (King, Koehane, and Verba 1994). To ensure consistency and address issues of inter-coder reliability, the author conducted all of the primary and secondary research. The following presentation of the analysis is in the same order in which the domains appeared in the individual cases. This delineation represents a parsimonious approach to presenting the analysis. A discussion of the analysis across the domains within this 
section reflects the desire to maintain the holistic and integrated nature of this study's analytical approach.

Leader-Client Co-Production Domain.

The theoretical model put at risk in this study posits that the existence of feedback loops and leading with a vision are the two independent variables measuring the antecedent of adaptive interest alignment. Table 14 presents the PSIOI cross-case analysis of adaptive interest alignment in the leader-client co-production domain while table 15 presents an analysis of this same antecedent in this same domain using the OAPSI rating methodology. Adaptive interest alignment is the antecedent that provides a pathway to innovation in the leader-client co-production domain. It manifests in the domain where the organization's leaders and their customers and clients co-produce innovation. Adaptive interest alignment serves to provide a causal pathway for the coproduction of innovation in the public sector.

Table 14. PSIOI Leader-Client Co-Production Cross-Case Analysis

\begin{tabular}{|c|c|c|c|c|c|c|}
\hline \multirow[t]{2}{*}{ Antecedent } & \multirow{2}{*}{$\begin{array}{l}\text { Variable: } \\
\text { High (+)/ Med } \\
(0) / \text { Low (-) } \\
\text { Measure }\end{array}$} & \multirow[t]{2}{*}{ Case } & \multicolumn{4}{|c|}{ Attributes } \\
\hline & & & Resilience & Quality & Accessibility & Efficiency \\
\hline \multirow{6}{*}{$\begin{array}{l}\text { Adaptive } \\
\text { Interest } \\
\text { Alignment }\end{array}$} & \multirow{3}{*}{$\begin{array}{l}\text { Existence } \\
\text { of feedback } \\
\text { loops }\end{array}$} & 1 & + & 0 & + & + \\
\hline & & 2 & + & + & + & + \\
\hline & & 3 & + & + & + & + \\
\hline & \multirow{3}{*}{$\begin{array}{l}\text { Lead with a } \\
\text { vision }\end{array}$} & 1 & + & + & + & + \\
\hline & & 2 & + & + & + & + \\
\hline & & 3 & + & + & + & 0 \\
\hline
\end{tabular}


Both the PSIOI and the OAPSI rating in the three cases reflect a positive association between the two independent variables and the co-production of public sector innovation. Twenty-two of twenty-four measures of the two PSIOI independent variables in the three cases were high. This is a very robust result. The two remaining empirical observations resulted in a medium measure. These medium measures related to quality in the first case and efficiency in the third case. These differences reflect naturally occurring variation in the data. In other words, the measures of the two attributes represented "noise" that fell short of providing a signal that the medium measure of these two attributes is meaningful.

\section{Table 15. OAPSI Leader-Client Co-Production Cross-case Analysis}

\begin{tabular}{|c|c|c|c|c|c|c|c|}
\hline \multirow[t]{3}{*}{ Antecedent } & \multirow{3}{*}{$\begin{array}{c}\text { Independent } \\
\text { Variable }\end{array}$} & \multicolumn{6}{|c|}{ Measure } \\
\hline & & \multicolumn{2}{|c|}{ Case 1} & \multicolumn{2}{|c|}{ Case 2} & \multicolumn{2}{|c|}{ Case 3} \\
\hline & & $\begin{array}{l}\mathbf{Y}(+) / \\
\mathbf{N}(-)\end{array}$ & $1 / 0$ & $\begin{array}{l}Y(+) / \\
N(-)\end{array}$ & $1 / 0$ & $\begin{array}{l}\mathbf{Y}(+) / \\
\mathbf{N}(-)\end{array}$ & $1 / 0$ \\
\hline \multirow{2}{*}{$\begin{array}{l}\text { Adaptive } \\
\text { Interest } \\
\text { Alignment }\end{array}$} & $\begin{array}{l}\text { Existence of } \\
\text { feedback loops }\end{array}$ & + & \multirow[t]{2}{*}{+1} & + & \multirow[t]{2}{*}{+1} & + & \multirow[t]{2}{*}{+1} \\
\hline & Lead with a vision & + & & + & & + & \\
\hline
\end{tabular}

The results of this case study indicate that the dependent variable of co-produced public sector innovation was present when the independent variables of the existence of feedback loops and leading with a vision were present. This reflects a positive correlation in the data. The data supports the contention that the existence of feedback loops and leading with a vision indicates the existence of adaptive interest alignment. This antecedent provides the path to the phenomenon within the leader-client domain. The 
antecedent of adaptive interest alignment serves as a pathway to the co-production of public sector innovation.

There was a high measure of the two independent variables in each of the three case's OAPSI rating. All six of the measures of the two independent variables indicating the presence of the antecedent of adaptive interest alignment were positive. This occurred across all three cases. This reflects the same positive correlation observed in the PSIOI. This is consistent with what the theoretic framework suggests. In other words, there is a positive correlation between the independent and dependent variables in this domain. The cross-case analysis revealed that in all three cases there was a high degree of alignment between the independent variables measured to construct the PSIOI and the OAPSI rating.

The next chapter presents the findings, conclusions, and recommendations that flow from the analysis contained in this section. One of the concerns the next chapter will address is the possible extraneous nature of the independent variable leading with a vision. This is the case since a concern has arisen over whether this independent variable is, in reality, a measure of the independent variable of transformational leadership. This relationship was unforeseen. It only became visible after a deep analysis of the case highlighted this potential discrepancy.

\section{Client-Organization Co-Production Domain.}

Table 16 reflects the cross-case analysis of the PSIOI for client-organization coproduction and table 17 depicts the analysis of the same domain using the OAPSI rating. Client-based prioritization is one of two antecedents that serves as a causal pathway to 
the co-production of innovation in the domain that exists between the organization and the customer or client. The premise is that too effectively function, an organization will engage in resource prioritization when it encounters a dwindling supply or stock of funding, staff, or other assets. In other words, a resource deficiency triggers a resource ranking. The two independent variables that measure client-based prioritization are the existence of resource constraints and the awareness of a co-production gap.

Table 16. PSIOI Client-Organization Co-Production Cross-Case Analysis

\begin{tabular}{|c|c|c|c|c|c|c|}
\hline \multirow[t]{2}{*}{ Antecedent } & \multirow{2}{*}{$\begin{array}{c}\text { Variable: } \\
\text { High (+)/ Med } \\
(0) / \text { Low (-) } \\
\text { Measure } \\
\end{array}$} & \multirow[t]{2}{*}{ Case } & \multicolumn{4}{|c|}{ Attributes } \\
\hline & & & Resilience & Quality & Accessibility & Efficiency \\
\hline \multirow{6}{*}{$\begin{array}{l}\text { Client-Based } \\
\text { Prioritization }\end{array}$} & \multirow{3}{*}{$\begin{array}{l}\text { Existence } \\
\text { of resource } \\
\text { constraints }\end{array}$} & $\overline{c 1}$ & + & 0 & + & + \\
\hline & & 2 & + & + & 0 & 0 \\
\hline & & 3 & + & + & + & 0 \\
\hline & \multirow{3}{*}{$\begin{array}{l}\text { Co- } \\
\text { production } \\
\text { gap } \\
\text { awareness }\end{array}$} & 1 & + & + & 0 & + \\
\hline & & 2 & + & + & - & - \\
\hline & & 3 & + & + & + & + \\
\hline \multirow{6}{*}{$\begin{array}{l}\text { Co- } \\
\text { Production } \\
\text { Readiness }\end{array}$} & \multirow{3}{*}{$\begin{array}{l}\text { Sunset } \\
\text { through co- } \\
\text { production }\end{array}$} & 1 & + & 0 & + & + \\
\hline & & 2 & - & 0 & - & - \\
\hline & & 3 & 0 & 0 & 0 & 0 \\
\hline & \multirow{3}{*}{$\begin{array}{l}\text { Information } \\
\text { symmetry }\end{array}$} & 1 & + & + & + & 0 \\
\hline & & 2 & + & + & + & 0 \\
\hline & & 3 & + & + & + & 0 \\
\hline
\end{tabular}

The PSIOI client-organization co-production cross-case analysis indicates that seventeen of the twenty-four observations of the independent variable across the three 
cases in the PSIOI category for client-based prioritization were high. Five were medium and two were low. There was a nearly even spread of medium measures across the attributes in the three cases related to the antecedent client-based prioritization in the PSIOI, although medium measurements predominate in the attributes of accessibility and efficiency. The two low ratings were associated with the second case in the areas of accessibility and efficiency. Other than the relatively lower ratings associated with the attributes of accessibility and efficiency, the results of this analysis generally appear consistent.

Table 17. OAPSI Client-Organization Co-Production Cross-case Analysis

\begin{tabular}{|c|c|c|c|c|c|c|c|}
\hline \multirow[t]{3}{*}{ Antecedent } & \multirow{3}{*}{$\begin{array}{l}\text { Independent } \\
\text { Variable }\end{array}$} & \multicolumn{6}{|c|}{ Measure } \\
\hline & & \multicolumn{2}{|c|}{ Case 1} & \multicolumn{2}{|c|}{ Case 2} & \multicolumn{2}{|c|}{ Case 3} \\
\hline & & $\begin{array}{l}\mathbf{Y}(+) / \\
\mathbf{N}(-)\end{array}$ & $1 / 0$ & $\begin{array}{l}\mathbf{Y}(+) / \\
\mathbf{N}(-)\end{array}$ & $1 / 0$ & $\begin{array}{l}\mathbf{Y}(+) / \\
\mathbf{N}(-)\end{array}$ & $1 / 0$ \\
\hline \multirow[t]{2}{*}{$\begin{array}{l}\text { Client-Based } \\
\text { Prioritization }\end{array}$} & $\begin{array}{l}\text { Existence of resource } \\
\text { constraints }\end{array}$ & + & \multirow[t]{4}{*}{+1} & + & \multirow[t]{4}{*}{+1} & + & \multirow[t]{4}{*}{+1} \\
\hline & $\begin{array}{l}\text { Co-production gap } \\
\text { awareness }\end{array}$ & + & & + & & + & \\
\hline \multirow[t]{2}{*}{$\begin{array}{l}\text { Co-Production } \\
\text { Readiness }\end{array}$} & $\begin{array}{l}\text { Sunset through co- } \\
\text { production }\end{array}$ & - & & - & & 0 & \\
\hline & Information symmetry & + & & + & & + & \\
\hline
\end{tabular}

An analysis of the distribution of the attributes that served to measure of two of the independent variables - existence of resource constraints and co-production gap awareness - indicates that the attributes of accessibility and efficiency yielded the greatest amount of variability. There was, moreover, no consistent pattern between the three cases. In this regard, the variation appears evenly spread between the cases. This is consistent with a random distribution of the attributes between the cases. The attribute of 
reliance had no ratings less than high. Quality had only one rating less than high, and that attribute was medium. This indicates a relatively robust measure of these two attributes across the independent variables although the attributes of accessibility and efficiency indicate areas of potential weakness.

With regard to the OAPSI index, the empirical observations resulted in a high measure for the independent variable existence of resources constraints. This occurred across all three cases. There is, likewise, a high measure of the independent variable coproduction gap awareness in the OAPSI index across all three cases. This reflects a robust measure of the two indicators of client-based prioritization. The robust measure of these two independent variables contributed significantly to the causal relationship between the antecedent of client-based prioritization and the dependent variable. In other words, this attribute served to create a pathway that allowed the dependent variable to emerge.

Co-production readiness is the second antecedent that serves as a pathway to public sector innovation. Sunset through co-production and information symmetry are the two independent variables that measure this antecedent. The PSIOI client-organization co-production cross-case analysis indicates that twelve of the twenty-four observations of the independent variables measured across the three cases in the PSIOI category for coproduction readiness were high. Nine were medium and three were low. The attributes of resilience, quality, and accessibility associated with the independent variables of coproduction readiness were the most robust across the three cases.

In the PSIOI, there was a great deal of ambiguity associated with the sunset through co-production independent variable. Six of the twelve observations resulting in a 
measurement of the attributes of this independent variable were medium, and two-thirds of these measurements were associated with the third case. Conversely, there were three high measurements of the attributes of this independent variable, with each relating exclusively to the first case. All three low measures of the attributes of sunset through coproduction were associated with Case 2. There was, therefore, evidence of variability between cases and consistencies within-cases. This, moreover, does not appear random in nature.

There was less ambiguity in the OAPSI rating regarding the independent variable sunset through co-production. This independent variable was measured as low within two of the three cases. In the third case, sunset through co-production was measured as medium within the case. It is possible to conclude from the PSIOI and OAPSI data that the independent variable sunset through co-production is irrelevant. The repercussions to the model and a discussion of the implications for how theorist, practitioners, and policymakers understand the phenomenon of co-produced innovation is in the next chapter.

The measure of the independent variable information symmetry was high across the attributes of reliance, quality, and accessibility. This occurred in all three cases related to the PSIOI. The notable exception was the efficiency category. In the efficiency category, all three measures of this attribute across the three cases was medium. Information symmetry in the OAPSI rating for all three cases was high. This leads to an overall high measure of information symmetry across all three cases. The overall high measure of information symmetry serves to confirm the validity of this independent variable. It is necessary, however, to account for the low measure for the attribute of 
efficiency. A relatively robust measure of information symmetry served to indicate the presence of the antecedent of co-production readiness. Along with the antecedent clientbased prioritization, this antecedent served to open the pathway to the co-production of innovation in the public sector.

Within the context of the PSIOI, the attributes of efficiency and accessibility measure the independent variables associated with the client-organization co-production domain. These measurements were consistently lower than the attributes of resilience and quality. The attributes of efficiency is a characteristic of New Public Management while accessibility is a hallmark of New Public Governance (Morgan et al. 2013). There was no observation of similar results in the leader-client or organizational-leader co-production domains. This suggests a role for leadership in developing products and services that are accessible and efficient. The findings, conclusions, and recommendations that result from the empirical observations as they relate to the client-organization co-production crosscase analysis contained in this section is the subject of the next chapter.

\section{Organizational-Leader Co-Production Domain.}

Three antecedents animate the domain of organizational-leader co-production. They are organizational structure and culture, organizational incentives, and risk-taking preferences. The model predicted that each of these antecedents contributed in some manner to creating a pathway to co-produced public sector innovation. Table 18 reflects the cross-case analysis of the PSIOI for the organizational-leader co-production domain and table 19 depicts the analysis of the same domain using the OAPSI rating. There were 
some unanticipated results in this domain. In several instances, the independent variables did not respond in the direction that the model predicted.

Table 18. PSIOI Organizational-Leader Co-Production Cross-Case Analysis

\begin{tabular}{|c|c|c|c|c|c|c|}
\hline \multirow[t]{2}{*}{ Antecedent } & \multirow{2}{*}{$\begin{array}{l}\text { Variable: High (+)/ } \\
\text { Med (0)/ Low (-) } \\
\text { Measure }\end{array}$} & \multirow[t]{2}{*}{ Case } & \multicolumn{4}{|c|}{ Attributes } \\
\hline & & & Resilience & Quality & Accessibility & Efficiency \\
\hline \multirow{6}{*}{$\begin{array}{l}\text { Organizational } \\
\text { Structure/ } \\
\text { Culture }\end{array}$} & \multirow{3}{*}{$\begin{array}{l}\text { Transformational } \\
\text { leadership }\end{array}$} & 1 & - & + & + & + \\
\hline & & 2 & + & + & + & + \\
\hline & & 3 & + & + & + & 0 \\
\hline & \multirow{3}{*}{$\begin{array}{l}\text { Ability to } \\
\text { experiment }\end{array}$} & 1 & - & - & - & - \\
\hline & & 2 & - & - & - & - \\
\hline & & 3 & 0 & 0 & 0 & 0 \\
\hline \multirow{3}{*}{$\begin{array}{l}\text { Organizational } \\
\text { Incentives }\end{array}$} & \multirow{3}{*}{$\begin{array}{l}\text { Incentives for } \\
\text { improvement }\end{array}$} & 1 & 0 & - & - & - \\
\hline & & 2 & 0 & - & 0 & 0 \\
\hline & & 3 & 0 & 0 & + & - \\
\hline \multirow{3}{*}{$\begin{array}{l}\text { Risk-taking } \\
\text { Preferences }\end{array}$} & \multirow{3}{*}{$\begin{array}{l}\text { Individual risk- } \\
\text { taking preference }\end{array}$} & 1 & - & - & - & - \\
\hline & & 2 & 0 & 0 & 0 & 0 \\
\hline & & 3 & 0 & - & - & - \\
\hline
\end{tabular}

In particularly, the case regarding the independent variables of individual risktaking preference, ability to experiment, and incentives for improvement did not respond in the anticipated direction. The resulting measures of all three of these independent variables have important implications for the model's validity. In several instances, the independent variables failed to indicate the presence of the antecedent. This was the case with the two variables ability to experiment and individual risk-taking preferences. In 
other instances, such as incentives for improvement, the research design required refinement. This refinement allows for a more accurate measure of the nuance of this independent variable. The implications of these empirical measurements are the subject of the concluding chapter.

Table 19. OAPSI Organizational-Leader Co-Production Cross-Case Analysis

\begin{tabular}{|c|c|c|c|c|c|c|c|}
\hline \multirow[t]{3}{*}{ Antecedent } & \multirow{3}{*}{$\begin{array}{l}\text { Independent } \\
\text { Variable }\end{array}$} & \multicolumn{6}{|c|}{ Measure } \\
\hline & & \multicolumn{2}{|c|}{ Case 1} & \multicolumn{2}{|c|}{ Case 2} & \multicolumn{2}{|c|}{ Case 3} \\
\hline & & $\begin{array}{l}\mathbf{Y}(+) / \\
\mathbf{N}(-)\end{array}$ & $1 / 0$ & $\begin{array}{l}Y(+) / \\
N(-)\end{array}$ & $1 / 0$ & $\begin{array}{l}\mathbf{Y}(+) / \\
\mathbf{N}(-)\end{array}$ & $1 / 0$ \\
\hline \multirow{2}{*}{$\begin{array}{l}\text { Organizational } \\
\text { Structure/ } \\
\text { Culture }\end{array}$} & $\begin{array}{l}\text { Transformational } \\
\text { leadership }\end{array}$ & + & \multirow[t]{4}{*}{0} & + & \multirow[t]{4}{*}{0} & + & \multirow[t]{4}{*}{0} \\
\hline & Ability to experiment & - & & 0 & & - & \\
\hline $\begin{array}{l}\text { Organizational } \\
\text { Incentives }\end{array}$ & $\begin{array}{l}\text { Incentives for } \\
\text { improvement activity }\end{array}$ & 0 & & 0 & & 0 & \\
\hline $\begin{array}{l}\text { Risk-taking } \\
\text { Preferences }\end{array}$ & $\begin{array}{l}\text { Individual risk-taking } \\
\text { preference }\end{array}$ & - & & - & & - & \\
\hline
\end{tabular}

The independent variables of transformational leadership and ability to experiment indicate the presence of the antecedent organizational structure and culture. There was a high degree of alignment between the PSIOI and OAPSI measurements of the independent variable of transformational leadership. Ten of twelve measurements of this independent variable in the PSIOI were high. The only deviation from this measure was reliance in Case 1 and efficiency in Case 3 that were low and a medium, respectively. The medium and low measurement differences reflect naturally occurring variations in the data. Furthermore, all three measures of this independent variable in the OAPSI rating were high. 
To ensure a robust assessment of the independent variable of transformational leadership, a single high measurement required the empirical observation of three or more instances of a transformational leadership trait. The definition of what constitutes a trait occurred prior to their empirical observation in the field. It is important to note that a high measurement required a minimum of one observation of three separate traits. As opposed to three observations of a single trait. This measurement protocol resulted in a high level of confidence that the measure of transformational leadership was valid. What is clear from the subsequent analysis of the data is that transformational leadership played a critical role in creating the conditions necessary for the co-production of innovation in the public sector.

Experimentation in the context of this dissertation is the process of conducting a limited innovative act to determine the efficacy of something previously untried. There was a low measure of the independent variable related to experimentation reflected in the PSIOI for Cases 1 and 2. This measurement was low in each of the four attributes of this independent variable in these two cases. In Case 3, there was a medium PSIOI measurement of each of the four attributes of this independent variable. Applying the OAPSI rating methodology to the cases yielded similar contradictory results. In the first and third cases, the measurement of the independent variable related to experimentation was low and measurement was medium in the second case study.

The measurement of the independent variable of ability to experiment was consistent across the PSIOI and OAPSI in Case 1. In other words, there was a positive alignment between the PSIOI and OAPSI such that the measure of the independent variable in the two indices fluctuated together. This did not occur in Cases 2 and 3. In 
Cases 2 and 3, the measure of the PSIOI and OAPSI rating offset. In Case 2, ability to experiment was measured low across all four attributes in the PSIOI. However, a medium measure of the variable occurred in the OAPSI. The opposite measurement occurred in Case 3. In this case, ability to experiment was measured as medium across all four attributes of the PSIOI, but was measured as low in the OAPSI. It is possible to conclude from the data that the ability to experiment is an irrelevant antecedent.

Incentives for improvement activities is the independent variable that measures the antecedent of organizational incentives to innovate. In six out of twelve empirical observations related to the PSIOI, there was an assignment of a medium measurement. There was a low measure five out of twelve times and a high measure occurred once. The measure of the independent variable that indicates the presence of the attribute of resilience was medium across all three cases. The measure of incentive for improvement tended to skew towards a medium measure for accessibility and a low measure for quality and efficiency across the three cases. For the OAPSI rating, the independent variable associated with the attribute of incentives for improvement activity was measured as medium in all three cases.

This is not consistent with what the model would predicted for the independent variable incentives for improvement activity. Further analysis of the individual cases is necessary to put the incentives for improvement activity independent variable and its measurement into perspective. A medium measure of this independent variable indicated evidence of either an intrinsic or an extrinsic incentive linked to innovation, but not both (see Appendix B). This was the basis for the OAPSI and PSIOI rating. In all three cases, 
there was strong evidence of intrinsic incentives related to innovation. Conversely, in all three cases extrinsic incentives for innovation were not present.

It is possible to attribute the skewing of the results of the measure of the attribute of incentives for improvement to the study's design or structure. In other words, the structure of the study resulted in a measure that on the surface appeared contradictory, but further analysis revealed the need to refine the model and method of measurement to account for a more nuanced result. In this regard, the design of the research question and the nature of the coding protocol served to provide a measure that appeared out of alignment with the model. The question did not have the necessary granularity to confront the seemingly contradictory response. This study was flexible and adaptive enough to explain this seemingly contradictory result. Upon closer analysis, it was possible to discern the true nature of this antecedent.

Individual preferences for risk-taking is the independent variable the model suggests indicates the presence of the antecedent of risk-taking preferences. The crosscase analysis for the PSIOI and OAPSI revealed alignment between the measurements of this independent variable in the low to medium direction across the three cases. In the PSIOI, seven of the twelve observations resulting in measures across the four attributes was low while the remaining five were medium. There were no high measures. This highlighted the tendency of the data to skew towards a low measure for this independent variable. In the PSIOI for Case 1, the measurement of this independent variable was low across all four attributes of the independent variable. This differed in Case 2, where the coding resulted in a medium value across all four attributes of this independent variable. Case 3 closely resembled the outcome of Case 1, with only the attribute of resilience 
receiving a medium measure. In the OAPSI rating, there was a low measurement across all three cases for this independent variable.

The model predicted that the presence of the antecedent of risk-taking preferences contributed to the opening of the pathway for co-production of public sector innovation. The empirical evidence does not support this contention. Indeed, the opposite appears to be the case. Co-production of innovation in the public sector manifests independent of an individual or organizational preference for risk. The empirical observations support the assertion that the appetite for risk in the public sector is an independent variable and skews towards risk avoidance. It is dependent, moreover, on conditions in the strategic environment.

Risk is multifaceted and broadly defined within the study population. This has important implications for the perception of risk in the public sector. The fiduciary duty of public servants to protect against the loss of common pool assets is an important component in the management of risk. It is possible, therefore, for special districts to engage in innovation and maintain a low tolerance for risk when compared to their counterparts in the private sector. It is possible to conclude from the empirical evidence that this independent variable is irrelevant.

The analysis of the empirical observations across the three cases served to highlight the importance of transformational leadership to fostering the conditions necessary for the co-production of public sector innovation. There was a robust measure of the independent variable transformational leadership across all three cases. There was little support for the contention that antecedents measured by the independent variables ability to experiment and individual risk-taking preference play a role in creating the 
conditions necessary for the co-produce public sector innovation. The results of the crosscase analysis indicated the need to refine the model's assumptions regarding organizational incentives. In this regard, there is strong evidence that intrinsic incentives play a role in creating the conditions for the co-production of public sector innovation.

\section{Conclusion}

This chapter contains a presentation of three cases in which the social phenomenon of co-created public sector innovation occurred. Each case presented in this chapter contained a description of the measurement of each independent variable using first the PSIOI and then the OAPSI rating methodology. This chapter also contains an analysis of the two indices associated with the three cases. The first index served to measure the expected level of public sector innovation. This was the PSIOI. The second index measured the actual level of innovation. This was the OAPSI rating.

These indices are summary measures of the dependent variable. Individuals engaged in the co-production of public sector innovation are the dependent variable. The independent variables served to operationalize the model and predict the manifestation of public sector innovation. The analysis of these indices occurred on two levels. The first level was a within-case analysis. Investigation at this level allowed individual patterns and causal relationships to emerge in the context of a single integrated social event. This analysis was the basis for the findings, recommendations, and conclusion presented in the next chapter. The analysis also resulted in the identification of emergent themes and topical insights. 
A single case represented a single observation of the phenomenon. There is a danger in relying on a single observation of a phenomenon to draw generalizable conclusions. For this reason, there were multiple cases included in this study. This allowed for multiple observations of the phenomenon. The second level of analysis contained in this chapter, therefore, was across the three cases. This has the benefit of allowing for a more robust validation of the model.

The intent of this study is to create and validate a model that provides an understanding of the social interactions that lead the co-production of public sector innovation and predicts the phenomenon. The selection of cases was deliberate and designed to support this effort. Most of the results of this analysis aligned with what the model predicted. This was not, however, universally the case. There were several instances where the empirical observations ran counter to what the model predicted. Thus, a number of the antecedents appeared contradictory or irrelevant.

What flows from the analysis contained in this chapter is the topic of the concluding chapter. The next chapter opens with a critique of the analytical approach and the data collection and analysis methodology employed in this dissertation. The chapter then details six principle findings that resulted from the analysis contained in this chapter. Integrated into the presentation of the findings are some conclusions and recommendations that flow from the findings. The next chapter also contains a discussion of the implications of these findings to the model. This includes recommendations regarding the model's validity and generalizability to other governance structures and sectors. 


\section{CHAPTER 6: CONCLUSION}

The legitimacy of applying the scientific method to an empirical research agenda in the social sciences is an oft-debated issue (Little 1991). Kirkpatrick (1977) asserts that empirical theory "is oriented to the existential world... the behavior of individuals as they participate in the political processes and institutions of society" (10). He suggests that the scientific approach in the social sciences refers to "work that seeks to develop empirical theory (1) capable of explaining a wide range of apparently discrete and disparate political phenomena and (2) capable of being tested and possibly disproved by further research" (Kirkpatrick 1977, 18, emphasis in original). In other words, actual objective observations serve as the basis for both describing and explaining the phenomenon.

This dissertation is an exercise in empirical theorizing that is scientific in nature and positivist in orientation. Popper (1959) argues that falsifiability characterizes empirical theorizing. The central principle of scientific inquiry; therefore, is that no empirical observation can prove a theory, but it can contradict one (Popper 1959). The approach taken in this dissertation to understanding the phenomena of co-creating public sector innovation represents public administration as science. As such, it is void of normative principles dealing with the ultimate goal of innovation. It suggests a plausible explanation for public sector innovation and provides a systematic and empirically verifiable understanding of why the phenomenon occurs in the manner it does. This dissertation, moreover, allows future researchers to duplicate the methodological approach and verify the results of this study. 
Empirical theories, it is important to note, differ fundamentally from normative theories. The latter is prescriptive and value-laden. Normative theory refers to a "judgement made with reference to criteria" and represents political science and public administration as philosophy (Kirkpatrick 1977, 14). It seeks to define what individuals "ought" and "ought not" do in the public sphere. Normative theories reflect the shared values or institutional artifacts that are constitutive of social structures. The challenge normative theories present the researcher is that they are impossible to test or verify (Popper 1959).

There is, nevertheless, a normative aspect to this research agenda. It embeds, for example, in the definition of public sector innovation. In the context of this dissertation, public sector innovation represents systemic behavioral change. The systemic behavioral change is that which results from practices imbedded in institutions. Institutions modulate the individual and the individual modulates the institution. The systemic behavioral change that results from this modulation, moreover, leads to innovation that both outperform previous practices and results in the common good.

\section{Introduction}

The findings, conclusions, and recommendations contained in this chapter find their foundation in empirical and normative theorizing about the phenomena of public sector innovation. Underlying this dissertation is an assumption of intuitionalism such that "action is a function of the meaning which the actor ascribes to the act" (Natanson 1977, 530). Intuitionalism provides the perspective from which this dissertation seeks to describe the phenomenon of public sector innovation in a way that is accessible to 
theorists and practitioners alike. This idea represents an exercise in applied research "designed to produce knowledge useful in altering a real-world condition or situation" (Johnson and Joslyn 1995, 18). This approach provides the way in which the presentation of the research results will proceed.

The case study approach is particularly well suited to the development of a theory that explains the phenomenon of public sector innovation. In the context of this dissertation, the case study method represents a "way of using data" to better understand complex phenomenon (Freeman 1977, 716). It allows for the expansion of the analysis beyond observable actions to include an exploration of the intentions, strategies, interpretations, and perspectives of the actors (Yin 1994). This permits, consequently, the proper analysis of observable actions. The case study method complements and accommodates a wide range of methodologies and is particularly well suited for the development of theory (Bock 1977). An important attribute of the case study is its timebound and contextual nature. This allows for a temporal understanding of the phenomenon.

This dissertation is an exercise in theory creation involving a complex and multifaceted social phenomenon. The task of this study, therefore, is to create a bridge between a theoretical model and the empirical research necessary to build the theory. This bridge will allow for an understanding of the interactions necessary to predict when the co-production of public sector innovation will occur. This chapter seeks to explore the linkages between the model and the empirical world in which it applies (Dubin 1969). The purpose of this exercise is to ground the model in the social world. It does so in a manner that is intensely practical for the theorist and practitioner alike. 
Dubin (1969) observes there are limits to a researcher's power of observation and comprehension. He concludes that the result is a selective approach to narrowing the field of observation the researcher will deal with from an analytical perspective. This could result in a limited, simplistic, or particularistic view that restricts our understanding of the phenomenon. The strategy for dealing with this challenge is a close analysis of the implications of this dissertation's methodological approach to the research and the corresponding philosophical approach to theory building. The methodological and philosophical approaches taken to studying the phenomenon of co-produced public sector innovation reflect two sides of the same proverbial coin.

This chapter opens with a critique of the analytical approach to the research. The next section of this chapter presents an appraisal of the data collection and analysis methodology. Together these two sections provide a critical evaluation of this dissertations research methodology that illuminates one side of the proverbial coin. The following section presents the findings, conclusions, and recommendations that flow from the analysis of the data presented in the previous chapter.

There are six findings offered in this chapter, and each relates to a single antecedent articulated in the original model. Each conclusion and recommendation relates to one of the findings. The next section contains a summary of the findings and the revision of the model based on the findings. The value of this study to the practice, theory, and public policy is the topic of the following section. This chapter then concludes with a presentation of future research opportunities that flow from this dissertation. 


\section{Analytic Approach}

The analysis strategy employed in this dissertation provides a holistic perspective through an inductive examination of the aggregate data. This results in a synthesis that yields useful insights into the co-production of public sector innovation. It accomplishes this by allowing for a thick description of social behavior (Geetz 1971). Thick description provides sufficient contextual meaning to place individual behavior into proper social perspective (Little 1991). The goal of which is to validate a theory (Dubin 1969). The analysis contained in this dissertation includes a critical assessment of the intended meaning of the available data. This is necessary in social science research to analyze the accuracy, trustworthiness, and sincerity of the data (Little 1991).

George and Bennett (2005) define a "case" as representing an instance of a "phenomenon of scientific interest" (17). In the context of this study, a case represents an occurrence of innovation in the public sector that is co-produced. A case is distinct from the case study method (Yin 1994). The latter represents a social sciences methodological approach in which a single or multiple cases are the subject of scientific inquiry. This can include a research design that involves a within-case examination of a single case and/or a cross-case comparison of multiple cases. The most epistemologically sound case study approach involves a research design that combines both single and cross-case comparison methods (George and Bennett 2005).

This dissertation consists of a series of integrated narratives. The purpose of organizing the case as an integrated narrative is to facilitate the presentation and exploration of the underlying principles. The postulation of the principles occurred during the theory-creation phase of this dissertation. The validation of these principles arose 
during the analysis of the data gathered during the field research phase. The integrated narratives function to organize and report the analysis of the data in an imaginative literary pattern. The organization and presentation of the analysis of the data using this approach is both logical and coherent. In the context of this dissertation, integrative narratives are the most effective way to present the analysis of the data.

The individual cases serve to illustrate what is meaningful to describe, explain, and understand. The integrated narratives embed within the individual cases. A narrative represents a descriptive and causal analysis of past events or facts. It assumes the perspective of a critical inquiry into a particular social phenomenon (Geertz 1971; Little 1991). The focus on descriptive analysis provides an understating of the social interactions that define the phenomenon (Dubin 1969). In turn, the emphasis of causal analysis is on predicting the outcome of the phenomenon (Dubin 1969). This dissertation's approach to the practice of theory building and research allows for both the descriptive and casual analytical foci of attention to exist in parallel.

This study involves interpreting the facts in a pattern that seems most significant to the proposition at hand (King, Keohane, and Verba 1994). The priority is on data that the subject emphasizes as most salient to their individual experiences with the coproduction of public sector innovation. The data and accompanying analysis support the proposition to the extent the data supports the proposition. If the data does not support the proposition then an alternate proposition will emerge (Singleton and Straits 2010). This would result from the primacy of alternate social phenomenon. In these circumstances, the integrated narratives would reflect the alternate scheme. 
The analytic approach taken in this dissertation is subject to a number of critiques. What follows in the remainder of this section is an exploration of the more salient criticisms. One possible criticism is that the approach is particularistic in nature. This critique suggests that the focus of the thick description of circumstances and relationships is solely on meaning, which implies that general knowledge is not possible from particular facts (King, Keohane and Verba 1994). King, Keohane, and Verba (1994) counter that it is possible to study systemic patterns in similar parallel events. This process reveals both general and specific knowledge of social phenomenon.

A second possible critique of this study is that there was no consideration given to evaluating the quality of the public sector innovation that is co-produced. This critique may have merit for two principle reasons. First, this assessment is potentially valid to the extent that this study views the customer or client of the organization through the lens of the public sector organization. Deming (1986) argues that, "Quality should be aimed at the needs of the customer" (5). Therefore, for example, there was no systematic attempt to interview customers or clients of the organization. The counter to this argument is that the existence of feedback loops between the leader, organization, and customer or client served as a proxy measure of quality. Quality was also one of the attributes assessed in relation to the independent variables.

Second, it is possible to argue that there was no assessment of the robust nature of the co-production of public sector innovation. This criticism relates to the sustainability or longevity of the public sector innovation. The underlying premise of this critique is that there are thick and thin instances of this social phenomenon (Geetz 1971). The assumption is that the former has greater value. This study assumed that the existence- 
thin or thick — of co-produced public sector innovation was sufficient for this theory creation exercise. It is possible for future research to take into account levels of quality when designing a research protocol and, thereby, definitively address this criticism.

The manner of operationalization provides a third possible critique. In other words, the propositional statements used to observe and empirically measure the variables were erroneous. It is possible, for example, that the research instrument improperly captured the independent variables. It is also possible that the manner of measurement improperly quantified the independent variables. The question arises over the most appropriate yardstick for measurement, and whether the measurement would more appropriately be in relation to other individuals or organizations in the public sector, or in the private sector.

King, Keohane, and Verba (1994) assert that "If quantification produces precision, it does not necessarily encourage accuracy" (44). The counter to this argument is that a carefully constructed and well-defined case will foster accuracy (Dubin 1969). King, Keohane, and Verba (1994) contend, “Good causal hypotheses are complementary to good description" (45, emphasis in original). It is possible, therefore, to counter this critique by constructing an in-depth case study that relies upon the systematic collection of identical data across carefully selected manifestations of the social phenomenon.

A fourth critique is the backward-looking nature of this dissertation. This implies that what is relevant today is irrelevant tomorrow. This assertion of temporal cogency has some validity. This is the case in a time when the evolution of institutions is speeding up and increasing communications magnifies the impact of globalization on public sector innovation. The counterargument is that this study is contemporaneous in nature. As 
such, it presents the recent past in a way that is salient for today's practitioner and theorist.

Fifth, it is possible to claim that the level of analysis in this dissertation pointed to deeper systemic sources. There is some validity to this argument. One potential weakness of any model is its tendency towards the oversimplification of a complex and nuanced social phenomenon. Dubin (1969) argues that the goal of simplification is to improve our understanding of complex social phenomena. This supports the counterargument that simplification is beneficial if theories are to improve our understanding of the causal relationships inherent in complex social phenomenon.

\section{Data Collection and Analysis Methodology}

Science is a social enterprise in which research serves to confirm or refute our understanding of social reality. It is possible to know social reality through the collection and analysis of data. This dissertation represents an effort to know the co-production of public sector innovation through facts collected for reference and analysis through empirical observation. These facts serve as the basis for validating the proposed model of this phenomenon. What follows in this section is a critique of the data collection and analysis methodology used in this dissertation. The focus of this section, therefore, is on the process of research.

The criticisms reviewed in this section have the potential to undermine the efficacy of this study's findings, conclusions, and recommendations. More importantly, they could challenge the validity of the model. This section addresses the merits and relevance of the most salient criticisms in a methodical manner. It begins with a 
methodological critique. The focus then shifts to exploring challenges to the internal and external validity of the model (Kumar 2011). These two typologies of validity present the greatest potential areas of risk to the value of the findings, conclusions, and recommendations of this study. This section also addresses potential issues of data reliability.

One potential critique of this study's methodological approach is that the principle sources of data are overly narrow and particularistic. This could be the case since the basis of most of the primary and secondary source material is the special district itself. Yin (1994) defines three data collection principles for the case study approach. Adherence to these principles ensures the integrity of the data collection methodology. This, in turn, serves to minimize the chances that the dataset will prove irrelevant. Yin's (1994) first principle is the necessity of multiple sources of evidence. This dissertation aligns with this principle by using a mix of primary and secondary source data. The depth of the data sources, moreover, complements the width of the primary and secondary sources.

The second principle that will result in the efficacy of the model is the creation of a case study database (Yin 1994). A database serves to ensure the capture and display of the coded data in such a manner as to facilitate careful analysis. This allows for the reliable recall of data for future reference as well. Yin's (1994) third principle is to maintain a clear chain of evidence throughout the research effort. This dissertation's adherence to these three principles served to guard against criticism that the data collection and analysis process was haphazard or undisciplined. An assertion to the 
contrary could call into question the reliability of the conclusions and undermine this dissertation's attempt to build and validate a model of the phenomenon.

Another possible critique is that this study lacks internal validity. This criticism is valid to the extent that the case study research approach used in this dissertation fails to avoid confounding independent variables (Kumar 2011). The potential criticism is that alternate independent variables not previously identified are operative; the outcome of which is an inaccurate understanding of the model's causal relationships. In this regard, the less chance for the existence of confounding variables the higher the internal validity of the study. In other words, internal validity will prevail to the extent the research was properly done. Internal validity, moreover, relates only to the study of causal relationships (Kumar 2011).

The next section of this chapter contains suggestions for refining the measures of the independent variables. In this way, it is possible to refine the model to improve its predictive validity. The results, nevertheless, indicate that the salient independent variables identified in the next section caused the dependent variable to emerge. There was no evidence of confounding variables. In other words, a change in the independent variables is responsible for the empirical observation of variation in the dependent variable. This provides positive assurance that the only differences between cases are those related to the independent variables identified in this dissertation.

Yin (1994) asserts that the dominant mode of comparative case analysis is pattern matching in instances of non-manipulative or non-controlled case studies. In a comparative case study approach, the nonequivalent dependent variable represents the pattern of the social phenomenon. Successful comparative case analysis is dependent on 
achieving internal validity under conditions of ceteris paribus (Pennings et al. 1999). Since this dissertation aligned with these conditions, the result is confidence in the internal validity of this study.

Another possible critique is that the case studies lack external validity (Kumar 2011). In other words, the case studies were too particularistic to allow for any generalization of the findings, conclusions, or recommendations. The sections of this chapter that follow address the generalizability of the research findings as they relate to the theoretical model this dissertation proposed and put at risk. This section, therefore, addresses external validity as it relates to the representativeness of the sample, appropriateness of the empirical setting, and pertinence of the research protocol.

The sample of cases is representative to the extent that it accurately reflects instances of the phenomenon. In this regard, the index and rating presented in this dissertation provided a good indication of how the entire population would behave under similar conditions. This research protocol, furthermore, has the benefit of limiting or negating the effect of misperceptions or biases inherent in the human condition. Efforts made to identify and eliminate or mitigate possible sources of bias served to validate the research protocol.

Seeking out a diverse mix of empirical settings serves to ensure a diversity of beliefs that can counter the criticism that the sample was not representative. Each of these three cases share a single empirical setting. It is not a setting, however, that is unique or particularistic. Many of the attributes of this empirical setting are common in other empirical settings. The section of this chapter discussing generalizability will cover this in more detail. It is possible to conclude, furthermore, that the research protocol is 
pertinent to the study of this phenomenon (Dubin 1969). This is the case since the only differences between the special districts relate to the independent variable.

Yin (1994) suggests that a high quality comparative case analysis consists of four chief components. The first is a cautious contemplation of all relevant evidence. The second is a careful consideration of all major rival interpretations. The third is to ensure the analysis addresses the most significant aspects of the theory. The fourth, and final, is that the analysis requires prior expert knowledge of the phenomenon. This dissertation adhered to all four components. The result is a high level of confidence in the external visibility of this study.

Another possible critique relates to the reliability of the data gathering and analysis process (Kumar 2011). King, Keohane, and Verba (1994) assert that it is possible to achieve reliability by applying the same procedure, in the same way, each time there is a repetition of the procedure. If a procedure is reliable, then a duplication of the methodology will produce the same results. Reliability implies that the researcher collects the same data each time and over repeated observations. The technique used in this study yielded the same outcome each time, which implies that this dissertation's results were reliable.

Reliability contributes to the achievement of internal and external validity to the extent the researcher is able to carefully explain and amplify each stage of the research process (Kumar 2011). This will allow for the future replication, evaluation, and duplication of the research. The roadmap to building and validating the theoretical framework for the co-production of public sector innovation presented in this dissertation assures the possibility of repeatability in the future. In other words, there is confidence 
that the same research protocol when used in a similar or possibly different empirical setting will yield the same results each time. This confidence is possible since the same research protocol yielded similar results when applied to three different cases.

Another possible critique is that an independent variable serves as a proxy measure of another independent variable or a characteristic of that variable. As this dissertation unfolded, it became evident that the independent variable leading with a vision was also a characteristic of the independent variable of transformational leadership. Designating leading with a vision as an independent variable had the effect of further amplifying the role of transformational leadership and producing an effect consistent with an extraneous variable.

There were also implications that arose from the mathematical approach to modeling the PSIOI and OAPSI rating. There was no attempt to weight the importance of one independent variable over another. This has the potential of amplifying or downgrading an independent variable's impact on the dependent variable. This is a minimal risk given the theory building nature of this dissertation-in theory-building, the amplitude of the signal is less important than the existence of the signal (Dubin 1969).

There was also no sensitivity analysis as it relates to the independent variables used to construct the PSIOI and OAPSI rating. Sensitivity analysis can provide insight into the apportionment of different sources of uncertainty related to the independent variables and their effect on the dependent variable. This is a minimal risk to the findings, conclusions, and resulting recommendations presented in the next section of this chapter because in theory building, uncertainty in apportioning the results is less important than the empirical observation of the presence or absence of the predicted outcome. 
The case study approach can produce rich explanations necessary to understand social interactions and predict outcomes (Yin 1994). The case study approach also contributes to the parsimoniousness of theory. There is, nevertheless, a danger of oversimplifying a complex social phenomenon. This is an inherent danger in the theorybuilding methodology used in this dissertation (Dubin 1969). The internal and external validity demonstrated in the case study approach contributed to the efficacy of this study and served to mitigate this risk. The reliability demonstrated in this section ensures the reproducibility of this study's results in the future. Reproducibility will permit future testing and further validation of this theoretical model.

\section{Findings, Conclusions, and Recommendations}

This section presents the findings, conclusions, and recommendations of this dissertation. Findings represent the principal outcomes of this study. They signify what the research suggested, revealed, or indicated about the model. As such, the findings relate directly to the validity and efficacy of the proposed theoretical model. Each of the findings outlined in this chapter attaches to one of the six antecedents that serve to open the pathway to co-production of public sector innovation. In the context of this dissertation, findings refer to the totality of outcomes that animate the theoretical framework. Findings orient, furthermore, on the model's domains. In this manner, they differ from this study's conclusions and recommendations. Specific conclusions and recommendations, however, flow from these findings.

Conclusions emerge from the findings. They serve to provide the presumption for the significance of the findings. They represent a claim about the necessity and 
sufficiency of the model's independent variables. In other words, the conclusions contained in this section represent the necessary conditions that result from two linked propositions. They serve as the concluding premise regarding the co-production of public sector innovation. In this manner, the conclusions reflect a reasoned judgement regarding the conditions under which the model is operative. The conclusions emanate from the findings in which general circumstances predictably imply general consequences (Kraft and Furlong 2013).

The recommendations in this chapter also spring from the findings. They relate either to a suggested modification of the model or to an activity that will promote the coproduction of public sector innovation. As to the former, the recommendations suggest changes that will improve the utility of the model. As such, the recommendations represent a suggestion or proposal as to the best course of action for improving the model's efficacy, typically involving the independent variables. The result is a revision to the original model that better explains this complex phenomenon.

Recommendations also suggest activities that will promote co-production of public sector innovation. In the context of this dissertation, recommendations reflect specific circumstances that allow for the inference of specific consequences that will result in a general outcome (Kraft and Furlong 2013). They represent a suggestion or proposal as to the best course of action based on what the research into the model implies. In other words, a recommendation indicates an activity that will promote the coproduction of public sector innovation.

Three common notions or thoughts animate a social scientist's reasoning around causality (Little 1991). The findings and conclusions in this section reflect all three of 
these notions. First, the pathway that opens within the domains of the model are a manifestation of the social mechanism that connects the cause and the effect (Little 1991). In other words, the antecedents create the conditions that allow for the coproduction of public sector innovation. Causality is manifest in the pathway the antecedents create for the co-production of public sector innovation. The focus of cause and effect in this dissertation is upon the relationship between the antecedent and the phenomenon.

The second notion is that a correlation exists between two or more independent variables such that the dependent variable changes with the independent variables (Kumar 2011). The theoretical model postulates unobservable mechanisms and structures. Claims about causality emanate from the theoretical explanation the model provided. The basis for this explanation is a hypothesis about the underlying mechanism of the social phenomenon of co-produced public sector innovation. In this study, the independent variables the theoretic model suggests serve as indicators of the antecedents take different values under different circumstances. More importantly however, is their tendency to vary together. The focus of correlation is on the relationship between the independent variables and the dependent variable.

The last notion of causality relates to the study's conclusions. It is the thought that one event is a necessary and/or sufficient condition for the emergence of another condition (Little 1991). The conclusions presented in this chapter make a distinction between necessary and sufficient conditions as they relate to the independent variables. The notion that one empirically observable incident is a necessary and/or sufficient 
condition for another focuses on the relationship between the independent variable, or the antecedent, and the dependent variable.

A necessary condition is one that must be satisfied in order to obtain another condition. In the context of this dissertation, the independent variable represents the condition that must be satisfied. If this condition is satisfied, then it is possible to assume the antecedent is present. The antecedent, in turn, opens the pathway to the co-production of public sector innovation. The converse is also true. Without a measurable observation of the independent variable, there is no chance for the co-production of public sector innovation since there is a presumption that the antecedent is absent.

The existence of the antecedent means that the co-production of public sector innovation is possible, but by no means guaranteed. In other words, with a measurable observation of the independent variable there is a chance for the co-production of public sector innovation. A sufficient condition exists if the event that the independent variable depicts guarantees that the phenomenon will occur. If an independent variable is necessary and sufficient then there is a guaranteed outcome. In other words, if the independent variable is necessary and sufficient, then the dependent variable of coproduced public sector innovation will emerge.

Table 20 summarizes the findings, conclusions, and recommendations of this dissertation. Each of the six sections that follow is devoted to one of the dissertation findings. Each section also presents the conclusions and recommendations linked to the individual finding. The findings, conclusions, and recommendations of this dissertation invite a review of previously unexplored research and a re-examination of familiar topics from a fresh perspective. The review of the research literature in chapter 2 served to 
Table 20. Study Findings, Conclusions, and Recommendations

\begin{tabular}{|c|c|c|}
\hline Findings & Conclusions & Recommendations \\
\hline \multirow{3}{*}{$\begin{array}{l}\text { 1. The independent variable } \\
\text { existence of feedback loops } \\
\text { creates the conditions necessary } \\
\text { for the antecedent adaptive } \\
\text { interest alignment to emerge }\end{array}$} & $\begin{array}{l}\text { 1a. The existence of feedback } \\
\text { loops is a necessary but not } \\
\text { sufficient condition for adaptive } \\
\text { interest alignment to exist }\end{array}$ & $\begin{array}{l}\text { 1a. Robust feedback loops } \\
\text { between leaders and their } \\
\text { customers or clients is critical to } \\
\text { the public sector innovation }\end{array}$ \\
\hline & \multirow[t]{2}{*}{$\begin{array}{l}\text { 1b. Leading with a vision is a } \\
\text { transformational leadership trait }\end{array}$} & $\begin{array}{l}\text { 1b. Modify model to specify } \\
\text { leading with a vision as a } \\
\text { transformational leadership trait }\end{array}$ \\
\hline & & $\begin{array}{l}\text { 1c. Modify model to eliminate } \\
\text { the independent variable labeled } \\
\text { leading with a vision }\end{array}$ \\
\hline $\begin{array}{l}\text { 2. The independent variables } \\
\text { existence of resource constraints } \\
\text { and awareness of a co- } \\
\text { production gap creates the } \\
\text { conditions necessary for } \\
\text { antecedent client-based } \\
\text { prioritization to emerge }\end{array}$ & $\begin{array}{l}\text { 2a. Individually, the existence of } \\
\text { resource constraints and an } \\
\text { awareness of a co-production } \\
\text { gap are necessary but not } \\
\text { sufficient conditions for client- } \\
\text { based prioritization to exist }\end{array}$ & $\begin{array}{l}\text { 2. Develop robust understanding } \\
\text { of existence of resource } \\
\text { constraints and awareness of a } \\
\text { co-production gap } \\
\text { across/between the organization } \\
\text { and customers \& clients }\end{array}$ \\
\hline \multirow{2}{*}{$\begin{array}{l}\text { 3. The independent variable } \\
\text { information symmetry creates } \\
\text { the conditions necessary for the } \\
\text { antecedent co-production } \\
\text { readiness to emerge }\end{array}$} & $\begin{array}{l}\text { 3a. The existence of information } \\
\text { symmetry is a necessary but not } \\
\text { sufficient condition for co- } \\
\text { production readiness }\end{array}$ & $\begin{array}{l}\text { 3a. Robust information symmetry } \\
\text { between the organization and } \\
\text { their customers/clients is critical } \\
\text { to public sector innovation }\end{array}$ \\
\hline & $\begin{array}{l}\text { 3b. Sunset through co- } \\
\text { production is neither a necessary } \\
\text { nor sufficient condition }\end{array}$ & $\begin{array}{l}\text { 3b. Modify the model to } \\
\text { eliminate independent variable } \\
\text { sunset through co-production }\end{array}$ \\
\hline \multirow[t]{2}{*}{$\begin{array}{l}\text { 4. The independent variable } \\
\text { transformational leadership } \\
\text { creates the conditions necessary } \\
\text { for the antecedent organizational } \\
\text { structure/culture to emerge }\end{array}$} & $\begin{array}{l}\text { 4a. Transformational leadership } \\
\text { is a necessary but not sufficient } \\
\text { condition for an innovative } \\
\text { organizational structure and } \\
\text { culture to exist }\end{array}$ & $\begin{array}{l}\text { 4a. Develop robust capacity for } \\
\text { transformational leadership }\end{array}$ \\
\hline & $\begin{array}{l}\text { 4b. Ability to experiment is } \\
\text { neither necessary nor sufficient } \\
\text { for an innovative organizational } \\
\text { structure and culture }\end{array}$ & $\begin{array}{l}\text { 4b. Modify the model to } \\
\text { eliminate the independent } \\
\text { variable labeled ability to } \\
\text { experiment }\end{array}$ \\
\hline \multirow{2}{*}{$\begin{array}{l}\text { 5. The independent variable } \\
\text { incentives for improvement } \\
\text { activity creates the conditions } \\
\text { necessary for the antecedent } \\
\text { organizational incentives to } \\
\text { emerge }\end{array}$} & $\begin{array}{l}\text { 5a. Intrinsic incentives are } \\
\text { necessary but not sufficient } \\
\text { organizational incentives }\end{array}$ & $\begin{array}{l}\text { 5a. Develop robust intrinsic } \\
\text { incentive programs }\end{array}$ \\
\hline & $\begin{array}{l}\text { 5b. Extrinsic incentives are } \\
\text { neither necessary nor sufficient } \\
\text { organizational incentives to } \\
\text { innovate }\end{array}$ & $\begin{array}{l}\text { 5b. Modify the model to specify } \\
\text { the existence of intrinsic } \\
\text { incentives (eliminate extrinsic } \\
\text { incentives) }\end{array}$ \\
\hline \multirow{2}{*}{$\begin{array}{l}\text { 6. The independent variable } \\
\text { individual risk-taking preferences } \\
\text { and the antecedent risk-taking } \\
\text { preferences are extraneous }\end{array}$} & $\begin{array}{l}\text { 6a. The antecedent risk-taking } \\
\text { preferences is neither necessary } \\
\text { nor sufficient }\end{array}$ & $\begin{array}{l}\text { 6a. Eliminate antecedent risk- } \\
\text { taking preferences from the } \\
\text { model }\end{array}$ \\
\hline & $\begin{array}{l}\text { 6b. The independent variable } \\
\text { individual risk-taking preference } \\
\text { is neither necessary nor sufficient }\end{array}$ & $\begin{array}{l}\text { 6b. Modify model to eliminate } \\
\text { the independent variable } \\
\text { individual risk-taking preferences }\end{array}$ \\
\hline
\end{tabular}


frame this dissertation and provided the empirical basis for the deductive creation of the model. The subsequent review of the literature contained in this chapter was narrower in scope. It is a targeted survey of the research useful in validating the theory and serves to explain and amplify the results of the analysis of the empirical data. Woven into the presentation of the findings, conclusions, and recommendations are the results of the subsequent review of the literature.

Finding 1: Adaptive Interest Alignment: The first finding of this dissertation is that the existence of feedback loops serves as an independent variable that correlates with the dependent variable of co-produced public sector innovation. The existence of feedback loops serves to indicate the presence of the antecedent adaptive interest alignment. This antecedent opens the pathway to the co-production of public sector innovation as a necessary, but not sufficient, condition. Adaptive interest alignment is manifest within the leader-client co-production domain. In this domain, the organization's leaders and their customers and clients co-produce public sector innovation. Feedback loops also serve to indicate a leader's preference for aligning their organization with the strategic environment.

Adaptive interest alignment reflects the elasticity necessary to orient the organization on achieving a common public good or service. It relates to the manner in which the adjudication of values occurs within an organization. Adaptive interest alignment reflects the degree to which an organizational leader can shape the future of the organization through the organizations relationship with its customer and clients. In this paradigm, the achievement of interests is subject to negotiation. Alignment in the context 
of this dissertation refers to a position of agreement or alliance with a customer or client of the organization. Feedback loops represent the politics of structure in which this interaction occurs (Kopell 2001).

The existence of feedback loops is one of the two independent variables that the original model suggested indicates the presence of the antecedent adaptive interest alignment. The second was leading with a vision. Van Acker and Bouckaert (2017) assert that feedback is a potential "foundation for the constant fine-tuning of public sector innovations" (3). Within the context of this dissertation, feedback loops represent instances whereby successful communication and consultation resulted in further communication and consultation.

Feedback represents the degree to which the pathway formed by an effect returned to the cause of that effect and successfully generated either more or less of the same effect. Feedback loops reflect the iterative nature of enabling and enacting innovation within the public sector (Koivisto et al. 2015). The existence of feedback loops provides a proxy measure for adaptive interest alignment. The implication is that feedback loops functions as an empirically observable measure of the existence of adaptive interest alignment. It reflects the fact that this antecedent is opening the pathway for the co-production of public sector innovation.

This finding led to the conclusion that the existence of feedback loops is a necessary but not sufficient condition for adaptive interest alignment to exist. This is the first conclusion of this finding. The empirical observations supported the contention of a positive correlation between the existence of feedback loops and the existence of coproduced public sector innovation. They did not support the contention that feedback 
loops alone were sufficient for the emergence of this phenomenon. Other independent variables played a role as well. In other words, the existence of feedback loops is one independent variable that helps to explain and predict the cause of public sector innovation.

Developing robust feedback loops between the leader and their customers and/or clients is critical to the emergence of co-produced public sector innovation. This is the first recommendation of this finding. The cases suggest a typology of feedback loops. They can exist, for example, on a formal or informal basis. Formal feedback loops are officially sanctioned or recognized. They are bureaucratic in nature. Established rules, procedures, or established social conventions govern their operation. Informal feedback loops, on the other hand, have a relaxed or unofficial style or nature. Feedback loops can also be tight or loose. They are tight to the extent they are robust in their frequency, regularity, and intensity. Loose feedback loops, conversely, are infrequent in nature and relatively superficial in manner.

The creation of the typology of feedback loops outlined above was the result of a careful analysis of the three cases. An example of a tight, formal feedback loop is the regular commission meetings held in all three of the port districts. At these meetings, the public could make comments to the commission on a wide variety of topics. User-group meetings involving customers and clients, on the other hand, focused on a particular topic or area of interests and were examples of tight, informal feedback loops. Examples of informal and tight feedback loops included day-to-day commercial interactions with established customers and clients. Social or ceremonial encounters with the public represented examples of informal and loose feedback loops. 
The second independent variable predicted to indicate the presence of the antecedent of adaptive interest alignment was leading with a vision. Ljungholm (2014) asserts that transformational leaders "require vision in order to enact change" (77). Wright and Pandey (2009) note that transformational "leaders must inspirationally motivate employees by clearly articulating an appealing vision of the organization's mission and future" (76). Van der Voet (2014) stresses that the theory of a transformational leader includes creating and articulating a vision that animates the organization towards future action.

Leading with a vision is a component of transformational leadership that allows others to perceive the objectives of their organization within the context of their own individual purpose, goals, or aspirations (Ljungholm 2014). This research led to the conclusion that leading with a vision is a transformational leadership trait and not a unique independent variable. As such, leading with a vision is a distinguishing quality or characteristic belonging to the transformational leadership paradigm. In other words, it serves to animate transformational leadership. In this context, leading with a vision is a component of the independent variable transformational leadership.

This process led to the recommendation to modify the model and eliminate the independent variable labeled leading with a vision. This is the second conclusion that flows from the finding presented in this section. Designating leading with a vision as an independent variable has an irrelevant and potentially undesirable effect on the theoretic framework evaluated in the three cases. Including leading with a vision as a separate independent variable serves to unduly amplify the phenomenon of transformational leadership and distort our understanding of the phenomenon of organizational innovation. 
Eliminating leading with a vision as an independent variable will serve to counter this undesirable effect.

Eliminating this independent variable has no impact on the efficacy of the model and improves the model's validity. In this context, removing this independent variable was shown to have no or minimal impact on the dependent variable. It also had no impact on indicating the presence of adaptive interest alignment. There is no evidence to support the connection, furthermore, between adaptive interest alignment and leading with a vision. In other words, the empirical evidence does not support the consideration of visioning within the context of adaptive interest alignment.

The research shows that the independent variable of transformational leadership serves to indicate the presence of organizational structure and culture within the domain of the organizational-leader co-production. This led to the need to modify the model to specify leading with a vision as a transformational leadership trait. This is the third and final recommendation of this finding. The identification of traits or characteristics of transformational leadership serve as a measure the independent variable of transformational leadership. The number of traits or characteristics empirically observed serves to indicate the robust nature of the measurement. This includes shared community values, inspirational leadership, and now leading with a vision (Bass 1985).

Finding 2: Client-based Prioritization: Client-based prioritization serves as one of two antecedents that creates the pathway to the co-production of public sector innovation within the domain of client-organization co-production. Co-production readiness is the other. Client-based prioritization reflects a customer-centric approach to public sector 
innovation. High levels of client-based prioritization indicate that the organization places a premium on identifying and implementing substantive improvements in the quality and quantity of the public goods and services it offers (Salge and Vera 2012). It is within this domain that the client and the organization create the conditions necessary for customercentered innovation to emerge (Salge and Vera 2012).

Customer-based prioritization reflects the degree to which public sector organizations place a priority on fulfilling the requirements of their customers or clients. This is salient because it is entirely possible for organizations to engage in innovation that has little value the perspective of the customer or client (Salge and Vera 2012). Clientbased prioritization reflects an organizational culture centered on meeting the requirements of the customer or client as opposed to activities that are the result of motives other than what customers or clients value (Salge and Vera 2012).

The antecedent client-based prioritization created a pathway through which the organization and the customer or client of the organization co-produced public sector innovation. Salge and Vera (2012) argue that "the customer orientation of the public service organization is likely to shape the extent to which that organization is able to translate innovation activity into tangible quality improvements" (552). This perspective links public sector innovation with the modality of co-production. The result is the generation of the conditions that cause the co-production of public sector innovation.

The second finding of this dissertation relates to client-based prioritization. The finding is that the independent variables existence of resource constraints and awareness of co-production gaps create the conditions necessary for the antecedent of client-based prioritization to emerge. Both independent variables suggest the need to prioritize scarce 
resources in the public domain. If there is an abundance of resources, there is little motivation to economize or make tradeoffs in resource allocation. In the face of unlimited resources, few gaps will exist, and those that do are easily closed. Unfortunately, in today's strategic environment, most public sector organization face conditions of scarcity.

The research data indicated that the dependent variable of co-produced public sector innovation varied positively with both of the independent variables that indicate the presence of the antecedent client-based prioritization. Client-based prioritization has a close link to the concept of customer orientation. Salge and Vera (2012) postulate that customer orientation serves to moderate the creation of public sector innovation. Their research, therefore, supports one of the principle conclusions of this study. Salge and Vera (2012) stop short, however, of claiming the existence of a causal relationship between customer orientation and public sector innovation.

The principle conclusion that emerged from the finding articulated in this section related separately to both of the independent variables. In the first instance, this study concludes that resource constraints are a necessary, but not sufficient, condition for the emergence of client-based prioritization. This is consistent with the findings of Salge and Vera (2012). The existence of resource constraints reflects cognitive dissonance between organizational perceptions with respect to the current condition of the organization and the desired or ideal state of the organization. It is in large measure imposed by the strategic environment in which the organization operates. It reflects an awareness of scarcity.

In the second instance, this study concluded that an awareness of a co-production gap is a necessary, but not sufficient, condition for the emergence of client-based 
prioritization to exist. The awareness of co-production gaps is a proxy measurement for the degree or extent to which the public sector organization is oriented to their customer and clients. The underlying assumption is that organizations will innovate to meet real or perceived needs of their customer or clients. These innovations, moreover, have a normative component since client-based prioritization will ensure public sector innovation is socially beneficial. In other words, the awareness of a co-production gap served to indicate a connection between the organization and its customer and clients essential to creating the conditions for the achievement of the common public good.

Salge and Vera (2012) advocate for making customer orientation a top priority for public sector organizations. This requires organizational leaders to establish a culture that values customer service. Doing so will contribute to the emergence of the dependent variable. Collectively, the existence of resource constraints and the existence of an awareness of a co-production gap create a necessary condition for client-based prioritization to exist. These two independent variables, therefore, couple to create the conditions necessary for this antecedent to emerge.

This leads to two principle recommendations. These recommendations emerge from the finding related to the antecedent of client-based prioritization. The first recommendation is the importance of developing a robust understanding of the existence of resource constrains between the organization and their customers and clients. The second recommendation is similar in nature; however, it relates to the co-production gap. In this instance, the recommendation focuses on the importance of creating a shared appreciation of the presence of co-production gaps across and between the organization and their customers and clients. 
These two recommendations, if followed, will have the effect of creating the conditions for the co-production of public sector innovation. It is important to note that this study assumes that the customer or client of the special district is pursuing the common public good. In other words, the common public good is inherent in the express wishes of the special district's customers or clients. This dissertation presumes, moreover, that to the extent this is not the case, democratic institutions (like the publicly elected port commission) will intervene to rectify the situation and enforce community values. In this manner, there is a presumption that institutional mechanisms will guarantee the achievement of the common public good.

The strategy for fostering the development of a customer-centric orientation within special districts differs depending on which independent variable the practitioner is attempting to influence. The locus of the independent variable indicating the existence of resource constraints has an orientation that is external to the organization. It results from a shared understanding between the organization and its clients as to what the client values. It is possible, furthermore, to know the existence of resource constrains through feedback loops.

The conclusion and recommendations related to this finding suggest the need for a robust network of feedback loops, but not necessarily for a particular typology of feedback loop. The requirement for a robust network of feedback loops links to the previous finding and serves to reinforce its salience. Developing a robust understanding of what is necessary to foster an understanding of the existence of resource constraints is contextual. Within the context of the cases explored in this dissertation, the feedback 
loops represent a conduit for the exchange of information. Their presence indicates the existence of a customer-centric orientation.

Morgan (2006) reasons that if a system is capable of employing feedback loops to obtain and analyze information about the efficacy of its action in the strategic environment, then it can alter its internal environment to generate actions that will improve the outcomes of its future activities. Unlike a perception of the existence of resource constraints, the locus of the independent variable indicating an awareness of a co-production gap is internal to the organization. It is congruent, however, with the perception of the existence of resource constraints. Co-production gap awareness results from organizational understanding and self-awareness. This self-awareness, in turn, serves as a trigger for the organization to embark on co-production activities with their customers and clients.

Walker (2013) observes that a perceived performance gap or a deviation from a norm will serve as a catalyst for implementing organizational change. The perception of a performance gap or a deviation from a norm serve to stimulate the emergence of a robust understanding of what is necessary to foster co-production of public sector innovation. An awareness of performance gaps across and between the organization and its customers and clients serves to create the conditions necessary for innovation. This awareness, in turn, is critical to improving the quality and quantity of innovation in the public sector. Salge and Vera (2012) argue that "the customer orientation of a public service organization is likely to shape the extent to which that organization is able to translate innovative activity into tangible quality improvements" (552). Prioritizing the needs of customers and clients is paramount to an organizations long-term viability. 
Finding 3: Co-Production Readiness: Co-production readiness is the second antecedent to inhabit the client-organization co-production domain. The other antecedent resident in that domain is client-based prioritization, discussed above. Co-production readiness reflects the degree to which the organization and their customers or clients are prepared to engage in co-production. It serves to open the pathway to the co-production of public sector innovation within the client-organization co-production domain. Information symmetry is the independent variable that indicates the presence of co-production readiness.

In the three cases examined, the dependent variable of co-produced public sector innovation was present when the independent variable of information symmetry was present. The same holds true for the antecedent of co-production readiness. In other words, there was a positive covariance between the dependent variable, the independent variable, and the antecedent. As discussed in the previous chapter, a prerequisite for the co-production of public sector innovation is a shared understanding of the actions necessary to close the gap between the current state and the ideal or desired future state. Co-production requires a shared conscience or understanding of what individuals and organizations are attempting to achieve. It also requires organizational and individual needs align on the objectives of co-production. Understanding how organizational and individual needs align with the needs of the special district's customers and clients is possible through an understanding of the phenomenon of information symmetry. In the context of this dissertation, the achievement of information symmetry is through the 
modality of feedback loops. Lewis et al. (2017) suggest that in this context feedback loops have the capacity to serve as mechanism of accountability and learning.

Information symmetry provides a foundation for ensuring the co-production of public sector innovation is contextually appropriate. Wright and Pandey (2009) suggest that horizontal and vertical communication mechanisms serve to create conditions under which transformational leadership can emerge. They suggest that the stronger these mechanisms, the more likely the chance that transformational leadership will flourish within an organization. Information symmetry, moreover, leads to the socialization and harmonization of values and goals. In this context, there is an alignment and internalization of the institutional norms of the special district alongside the values of the customer and client. This has the added benefit of breaking down perceived barriers to change.

It is possible to achieve information symmetry when the organization and its customers and clients share a mutual understanding of the need for co-produced public sector innovation. The degree of mutuality determines the level of symmetry. In other words, information symmetry exists to the degree to which a common, shared understand exists between individuals within the special district and their customers and clients. This mutual understanding, moreover, is the result of cognitive congruence over conjoined organizational outcomes. This shared understanding becomes the basis for mutual action, including the co-production of public sector innovation.

The principle finding related to the antecedent of co-production readiness is that the independent variable information symmetry created the conditions necessary for the antecedent co-production readiness to emerge. In other words, co-production readiness 
served to create the conditions necessary for and open the pathway to the dependent variable of co-produced public sector innovation to emerge. The existence of information symmetry served to indicate the presence of this antecedent.

Lewis et al. (2017) argue that the higher the frequency and quality of communications external to the organization, the more robust the network of feedback loops. The analysis of the data suggests this is the case for co-production of public sector innovation. This study finds evidence of the need for a robust network of feedback loops across all typologies capable of sustaining the rigors of co-production if innovation is to occur in the public sector. Lewis et al. (2017) assert that, "Social networks based on interpersonal communication generate embedded resources such as social capital and trust relationships" (5). Therefore, high levels of social capital and trust are necessary preconditions for individuals to engage in the co-creation process.

Two conclusions flow from this finding. The first conclusion is that the existence of information symmetry is a necessary, but not sufficient, condition for the emergence of the dependent variable. Information symmetry served to indicate the readiness of the special district and their customers and clients to engage in co-production. In the case studies, feedback loops were central to achieving information symmetry. As the three cases demonstrate, it is possible to leverage information symmetry to achieve consensus for action. In each of the three cases, robust networks of feedback loops existed and operated in relation to the observed innovations.

This conclusion recommends the development of robust mechanisms to achieve information symmetry between the organization and their customers and clients. This is a requirement if co-produced public sector innovation is to emerge. Feedback loops are 
characteristic of cybernetic organization systems (Scott 2003). In this regard, the organizational emphasis is on regulating routinized operations (Scott 2003). In the context of this organizational framework, feedback loops serve to detect departures from established goals. This is consistent with the earlier finding related to adaptive interest alignment. In this regard, feedback loops are part of the social structure. They serve as the proverbial plumbing. What these proverbial pipes carry are the stuff of collective social relationships necessary for co-production.

In perceiving divergence from existing aspirations, feedback loops served to trigger control mechanisms within the organization to stimulate action. Ingram et al. (2007) argue that path dependency characterized by increasing returns confer benefits to advantaged groups. Special district customers and clients are advantaged groups to the extent that they enjoy a social situation or prospect for securing especially favorable or desirable end states that lead to the achievement of their interests. This is consistent with the empirical observations made across the three cases. The phenomena of path dependency and feedback loops serve to explain the readiness of individuals and organizations to co-produce public sector innovation.

The second conclusion it is possible to derive from this finding is that the requirement to sunset an existing good or service is not a necessary or sufficient condition for co-producing public sector innovation. It is possible, therefore, to conclude that the independent variable sunset through co-production is an irrelevant variable. This independent variable is immaterial to the extent it does not serve to predict the presence of the dependent variable. Omitting this independent variable will have no impact on the model. While its inclusion did not necessarily bias the model, it could have served as a 
source of random variation. Irrelevant variables serve to either amplify or dampen the observed outcome in an undesirable manner.

What these three cases demonstrate is the salient nature of path dependencies and increasing returns in the public sector. Once a program or project is established, there is often little appetite for the subsequent termination of a product or service. Since the program or policy of the special district confers benefits on a particular group, that group subsequently becomes a constituency for its ongoing maintenance (Ingram et al. 2007). Unless other more powerful and politically connected constituencies view a project or program as a threat, there is subsequently very little reason to incur the political cost of its termination.

An analysis of the PSIOI for client-organization co-production reveals that there was a relatively high measure of sunset though co-production in the first case study, a low measure in the second case study, and a medium measure in the third case study (see table 16 on page 285). It is very difficult to ascertain a clear signal from such a dataset. As a result, the PSIOI lacked veracity in explaining or predicting the phenomena of sunsetting. The OAPSI provided a more robust measure in this regard. In the first two cases there was a low measure of the independent variable sunset through co-production (see table 17 on page 286). The third case revealed a medium measure of this independent variable.

The second conclusion was to modify the model to eliminate the independent variable labeled sunset through co-production. The supposition is that the independent variable of sunsetting related to existing projects or programs is irrelevant. It is possible to infer this from the results of an analysis of the indices in the three case studies. 
Eliminating the sunset through co-production independent variable strengthened the model by creating a less ambiguous indication of co-production readiness. It is worth considering, however, the social phenomenon of sunsetting within the context of the strategic environment.

The sunset phenomenon is a component of the strategic environment that operates as an externality on the model. Sunsetting, as a component of the strategic environment, works outside the model to influence the model's outcome. Sunsetting results from an activity or activities that occur within the context of the political economy in which the port district embeds. In this regard, sunsetting could result from actions by unrelated third parties. Sunsetting has either a positive or a negative impact on the interests of the special district and their customers or clients.

This study assumes that individual actors are operating in alignment with the rational actor model (Scott 2003). They operate, however, within an institutional context. The study also assumes that a customer or client is a willing partner in the co-production of public sector innovation. This is the case because it is possible to assume that since innovation is occurring to benefit the customer or client, the customer or client will act in a manner that maximizes their individual utility. The co-production of public sector innovation gives the opportunity for the customer or client to maximize their value. In other words, if customers and clients know their interests, and if they are capable of acting in a rational manner to achieve those interests, then they will engage in coproduction activities with public sector organizations. 
Finding 4: Organizational Structure and Culture: Organizational structure and culture is one of three antecedents that open the path to the co-production of innovation in the public sector within the organizational-leader co-production domain. The other two antecedents active in this domain are organizational incentives and risk-taking preferences. According to the model, the independent variables of transformational leadership and the ability to experiment indicate the presence of an organizational structure and culture that supports the co-production of public sector innovation. The empirical observations in the three case studies confirms that this is the case regarding transformational leadership; however, this is not the case regarding risk-taking preferences.

Organizational structure refers to the arrangement of the parts, elements, or constituents of an organization (Perrow 1986). It defines the way the organization directs activities towards the achievement of specific goals and objectives (Scott 2003). Mintzberg (1983) argues that this includes the allocation, coordination, and supervision of organizational missions. Lunenburg (2012) suggests that the inclusion of technical specialists in the operating core of an organization leads to rapidly innovating organizations that easily adapt to changing environments. Organizational structure also provides an individual or collective perspective for viewing the organization and its environment (Morgan 2006).

Organizational culture consists of the normative rules that regulate the manner in which individuals relate to one another within an institutionalized setting (Scott 2003). It represents a system of shared beliefs, values, and assumptions that govern individual behavior within the organizational structure (Morgan 2006). Organizational culture 
serves to moderate individual behavior within a social context (Rainey 1997). Mintzberg (1983) suggests that organizational culture represents the milieu of beliefs, traditions, norms, and values that imbed within the organization. It serves as the basic operating schema of the organization (Mintzberg 1983). Organizational culture, moreover, reflects in an organization's structure (Morgan 2006).

Organizational structure and organizational culture are two sides of the same proverbial coin when it comes to the co-production of public sector innovation. Together, they create the necessary conditions for opening a pathway to the co-production of public sector innovation. The empirical observations support the contention that organizational structure and culture exist as artifacts of institutionalized values embedded at the time of the organization's founding and subsequently reinforced by its customers and clients (Morgan 2006). Organizational structure and culture represents the proverbial social hardware and software of the organization that leaders are empowered to execute in the achievement of the organization's mission.

It is possible to conclude from the empirical evidence that leaders exert a powerful influence in shaping the structure and culture of an organization. This is consistent with the literature (Scott 2003; Morgan 2006). Indeed, Lewis et al. (2017) suggest that in the public sector "leadership has a bigger effect on innovation capacity than the structures, processes and contextual factors that drive innovation" (1). Walker et al. (2011) envision the leader's role as an individual entrepreneur pushing for institutional change. The empirical observations in all three cases strongly supported the contention that leadership plays a pivotal role in fostering co-production of public sector innovation. 
The literature on transformational leadership stresses its role in public sector innovation, although the link is not well established (Van der Voet 2014; Lewis et al. 2017). In the three case studies, the presence of the independent variable of transformational leadership aligned with the dependent variable of co-produced public sector innovation. This alignment was robust in relation to the independent variable of transformational leadership. This was not the case with the independent variable of experimentation. The cross-case analysis indicates the independent variable of experimentation did not respond in the anticipated direction. In fact, it skewed in the neutral to opposite direction of the dependent variable.

The fourth finding of this study is that the independent variable of transformational leadership created the conditions necessary for the antecedent of organizational structure and culture to emerge. The cases demonstrated that leadership was a key driver in fostering the co-production of public sector innovation. The evidence that emerged in all three cases served to support this relationship. This finding is also consistent with the literature on transformational leadership at the nexus of organizational structure and organizational change (Ljungholm 2014; Van der Voet 2014). What the case studies demonstrated was that transformational leadership is essential to the creation of a culture of trust, transparency, and enthusiasm necessary for the co-production of public sector innovation.

This is not to disregard the importance of transactional leadership. The cases demonstrated that transactional leadership has a role in situationally appropriate circumstances. Indeed, Orazi et al. (2013) note that attention in the research literature is shifting towards an "integrated" leadership perspective (494). In this perspective, 
"integrated" leaders use a combination of transformational and transactional behaviors" (Orazi et al. 2013, 494). This represents a shift away from a strictly dyadic differentiation towards a more holistically nuanced view of public sector leadership (Orazi et al. 2013).

The case studies demonstrate that transformational leadership is a necessary, but not a sufficient, condition for co-production of public sector innovation. This is the first conclusion to emerge from the finding related to the antecedent of organizational structure and culture. Transformational leadership empowers individuals and creates a strong sense of organizational identification (Ljungholm 2014). Organizational structure, in other words, provides the context for the exercise of transformational leadership. Transformational leadership shapes the organization's structure and culture. Conversely, organizational structure and culture shape the exercise of leadership.

Developing a robust capacity for transformational leadership is one of the two principle recommendations to emerge from the finding articulated in this section. The capacity to engage in the co-production of public sector innovation is dependent, moreover, on an organizational commitment of resources. This commitment requires an infusion of financial and human capital into the effort to co-produce public sector innovation. Case 1 is illustrative in this regard and suggests a possible approach to developing this capacity. In that case, the organization appointed an individual to work cross-functionally within the organization to foster the conditions necessary for innovation. In addition to human resources, the organization provided financial resources. In this case, the marketing budget became the vehicle for delivering organizational funding to innovate. 
The ability to experiment was neither a necessary nor a sufficient condition for an innovative organizational structure and culture to emerge. This is the second conclusion relating to this finding on the antecedent of organizational structure and culture. Experimentation means trying out new ideas, methods, or activities with an eye toward future widespread adoption if successful, or termination if not. The evidence suggests that the subjects perceived a level of risk was associated with experimentation. It appeared that the perception of risk related to the potential termination of the port's experiments.

In each of the three case studies, the failure of public sector innovations would result in an organizationally unacceptable loss of financial, human, or political capital. Experimentation, therefore, represented an exercise in risk. In these cases, moreover, the organizations exhibited a low level of risk tolerance. This further dampened enthusiasm for experimentation. The historical record of experimentation at the state and federal level, moreover, has a negative social connotation. For these reasons, experimentation was something the organizations in this study tended to avoid. As a result, the second recommendation to emerge from the fourth finding is the need to modify the model to eliminate the independent variable labeled ability to experiment.

It is possible that the subjects conflated the concepts of risk and experimentation such that each could result in negative outcome. This could be the case because risk and experimentation make explicitly the potential for loss. In the three case studies, innovators were attempting to close existing gaps in the port's offering of public goods and services. The organizations were looking for relatively permanent solutions to existing challenges. Innovation occurred in a resource-constrained environment in organizations that were largely risk adverse. There was, moreover, very little appetite for 
abandoning a solution once implemented, which is a possible outcome of experimentation.

Finding 5: Organizational Incentives: According to the model, organizational incentives are the second antecedent that is operative in the organizational-leader co-production domain. This antecedent opens the path to innovation in the public sector within the organizational-leader co-production domain. The previous section outlines the finding that relates to organizational structure and culture, which is one of the other two antecedents within the organizational-leader co-production domain. The next finding in this chapter will deal with the third antecedent in this domain-risk-taking preferences. The model suggests that the independent variable of incentives for organizational change indicates the presence of the antecedent of organizational incentives.

There was a medium measure of the independent variable of incentives for improvement activity when the dependent variable of co-produced public sector innovation was present. There was, in other words, alignment between the two variables. It was in the direction the model predicted, but not the magnitude that the model forecast. The theoretical framework suggested the measure of the independent variables should have been high in the presence of the dependent variable. The results, therefore, were not in total alignment with what the model predicted. This outcome requires further elaboration.

The fifth finding of this study is that the independent variable incentives for improvement activity creates the conditions necessary for the antecedent of organizational incentives to emerge. This antecedent opens the pathway to the co- 
production of public sector innovation. It accomplishes this by creating incentives that motivate individuals to co-produce public sector innovation. Most motivational theories tend to differentiate between intrinsic and extrinsic factors (Maslow 1943; McGregor 1957). These factors, in turn, serve as the basis for creating organizational incentives to behave in a certain manner.

Intrinsic incentives, within the context of the three case studies, represented rewards that came from within the individual. These rewards typically manifested upon completion of a task. In other words, the motivation was an individual's own satisfaction. Individuals receiving intrinsic rewards valued the challenge of the endeavor and found meaning in the undertaking. The rewards tended to support the individual's need for esteem or self-actualization (Maslow 1943). This is not to suggest that the organization had no role in providing intrinsic incentives. Indeed, the organization was capable of providing the conditions that enabled and amplified intrinsic incentives, including actions undertaken by the organization that produced inspirational motivation, granted individual autonomy, or provided intellectual stimulation.

In this dissertation, the presence of extrinsic organizational incentives would manifest as contingent financial rewards. They would relate, moreover, to the conduct of innovation. Extrinsic incentives would be external to the task themselves and support the fulfillment of physiological needs (Maslow 1943). They are external in the sense that other individuals largely control the distribution of the reward. Intrinsic incentives represented rewards that were tangible or physically provided to an individual in exchange for their accomplishing a specific goal or objective. The expectation is that the quantification of the reward would occur in advance of its award, to motivate the 
individual, and that the reward tied to specific behaviors the organization or its leadership wished to encourage.

The empirical observations of the independent variable of incentives for improvement activity measured the presence of both intrinsic and extrinsic incentives. Maslow (1943) suggests a hierarchy of needs in which individuals first seek to satisfy extrinsic needs prior to moving on to the satisfaction of intrinsic needs. Extrinsic rewards represent monetary compensation necessary to fulfil the lower level essentials according to Maslow's (1943) hierarchy. Intrinsic rewards, moreover, tie to the achievement of higher-level needs. McGregor (1957) argues that a satisfied need is not a motivator of behavior. This suggests that the intrinsic rewards inherent in public service will not motivate innovation.

Intrinsic incentives represented a necessary, but not sufficient, condition for the existence of organizational incentives to innovate. This is the first conclusion regarding the finding around organizational incentives. Intrinsic incentives represented a form of individual compensation for supporting organizational aims. In large measure, intrinsic incentives represented the psychological rewards that individual members of the organization received through the accomplishment of a meaningful task, making significant progress towards completing a task, or doing a task especially well. Intrinsic rewards were intangible in nature and satisfied intrinsic factors important to the individual.

The conclusion that intrinsic incentives is a necessary condition for the emergence of the dependent variable is consistent with the research literature on public sector innovation. For example, Orazi et al. (2013) suggest that organizational outcomes are 
dependent on the extent the public sector leader is capable of "increasing followers" intrinsic motivation, recognizing accomplishments, and rewarding high performance" (494). There is far less managerial discretion regarding compensation in the public sector compared to the private sector (Delfgaauw and Dur 2010). Monetary compensation, moreover, tends to be higher in the latter compared to the former (Delfgaauw and Dur 2010). Besley and Maitreesh (2005) suggest that mission preferences based on intrinsically satisfying assignments plays a key role in managers self-selecting into public sector employment.

The importance of the role of intrinsic incentives is fostering public sector innovation leads to the recommendation to create a robust program of intrinsic incentives within the public sector organization. As these case studies demonstrated, there is a wide range of intrinsic rewards at the disposal of the public sector organization. While the focus of intrinsic rewards is inward, there are actions that organizations can take to foster a robust program of intrinsic incentives. These include a focus on providing individuals the opportunity to participate in meaningful opportunities to engage in the co-production of public sector innovation. In addition to meaningful opportunities, providing individuals with choice or control over how they co-produce public sector innovation provides an intrinsic incentive as well.

Choice can also include the flexibility to achieve a balance between an individual's public and private lives. This includes the choice over when and where the individual may elect to engage in the co-production of public sector innovation. Another form of intrinsic incentive is the ability to gain mastery or competency in a particular area. The co-production of public sector innovation provided opportunities to accomplish 
this as well. In addition to meaningfulness, choice, and competency is providing individuals a sense of progress. Progress provides individuals a feeling of accomplishment. This sense of accomplishment can include public or private recognition of the individual's efforts around public sector innovation.

Extrinsic incentives are neither necessary nor sufficient organizational incentives to innovate. This is the second conclusion regarding the organizational incentive finding. Delfgaauw and Dur (2010) suggest that increasing monetary compensation is not an effective strategy for achieving organizational results in the public sector. Herzberg (1968) includes extrinsic incentives linked to monetary compensation as a hygiene rather than motivating factor. Thus financial remuneration is sufficient to maintain job satisfaction, but insufficient to motivate individuals. This was consistent with the empirical observations.

Potts and Kastelle (2010) approach the creation of incentives to innovate in the public sector from an economic perspective. In this regard, the principle of efficiency is their touchstone. In this context, the public sector organization "seeks to minimize waste and maximize deliverables from a finite budget" (Potts and Kastelle 2010, 124). Intrinsic rewards align closely with this objective. In each of the three cases, the organizations structured rewards for innovation around intrinsic incentives. The organizations in this study relied upon merit-based, equity-focused financial compensation systems that provided minimal discretion to senior leaders in providing individually tailored financial rewards. On the other hand, senior leaders had wide latitude to leverage intrinsic incentives to achieve organizational results. 
The conclusion that extrinsic rewards are not a necessary condition for the emergence of public sector innovation but intrinsic rewards are, leads to the recommendation to modify the model to eliminate the focus on extrinsic incentives. This is not to say that extrinsic rewards are not important. In fact, they were necessary to maintain support for organizational initiatives. Extrinsic incentives tied closely to maintaining sufficient satisfaction to achieve the core mission of the public sector organization.

These case studies demonstrated that extrinsic incentives are not as salient in spurring individuals to the high levels of motivation necessary to foster the co-production of public sector innovation. This modification of the definition of the independent variable to focus on intrinsic incentives will have the effect of creating more fidelity in the signal produced by the independent variable related to organizational incentive. The outcome of this modification is a more robust understanding of the causal mechanisms leading to the co-production of public sector innovation.

Finding 6: Risk-taking Preferences: A preference for risk-taking is the third antecedent to inhabit the client-organization co-production domain of the model. The theoretical framework for the co-production of public sector innovation suggests that the independent variable of individual risk-taking preference indicates the presence of the antecedent of risk-taking preference. The model put at risk in this dissertation, furthermore, suggested that this antecedent would open the pathway to the co-production of public sector innovation. The empirical evidence did not support either of these claims. 
In fact, there were high levels of risk aversion measured across each of the three cases. This is contradictory to what the model suggested.

The independent variable of individual risk-taking preferences did not align with the presence of the dependent variable. There was a low measure of risk-taking preferences across all three case studies. In other words, it was possible to conclude that high levels of individual risk aversion were present when the dependent variable of coproduced public sector innovation was manifest. These levels of risk aversion and risktaking preferences were not consistent with the model. The model predicted a high measure of this independent variable. This suggests the need for a greater understanding of the phenomenon of risk. While this result did not invalidate the model, it does suggest the need to modify the theoretical framework.

There was a PSIOI rating created for the twenty cases that made up the candidate pool of public maritime ports. As noted earlier, the assignment of a negative PSIOI led to the rejection of the case from further consideration. In light of the results of the analysis related to the empirical observation of individual risk-taking preferences, there was a discrete analysis of the independent variable of individual risk-taking preferences across all twenty cases with a PSIOI rating. This additional or supplemental analytical step was unique with no other cross-case analysis conducted involving any other independent variable across the PSIOI rating data.

The supplemental analysis conducted across all cases with a PSIOI rating indicated that there was a low value assigned to the independent variable in each of the twenty cases with a PSIOI rating. In other words, the cross-case analysis failed to identify a single instance of a medium or high rating related to the independent variable 
of risk-taking preferences within any of the PSIOI ratings conducted, including the three case studies in this dissertation. This supplemental analysis presented compelling evidence that all candidate cases involving public maritime ports in the Pacific Northwest shared a common feature related to risk-taking preferences.

If the independent variable of individual risk-taking preferences was low or nonexistent, then by extension the antecedent of risk-taking preferences was not operative. The fifth and final finding of this dissertation, therefore, is that the antecedent risk-taking preferences is extraneous. This is the case because the independent variable indicates the presence of the antecedent and, since the antecedent is extraneous, the independent variable is extraneous as well. The antecedent is extraneous to the extent that it does not provide an explanation or prediction of the phenomenon. In other words, the antecedent and its independent variable does not exist in the presence or absence of the dependent variable. This does not mean, however, that risk-taking preferences are iniquitous. Indeed the literature suggests they play an important role in the co-production of public sector innovation (Buurman 2012; Micheli et al. 2012).

The lack of alignment between the antecedent and the dependent variable with what the model would predict suggests an alternate explanation of the phenomenon. Several studies explore differences in risk-taking preferences between public and private sector employees. The literature suggests that individuals self-select into the private sector based on a preference for risk (Bellante and Link 1981; Buurman 2012; Micheli et al. 2012). In this regard, the more risk aversion, the greater the probability of selfselecting a career in public service. Bellante and Link (1981) conclude, for example, that individuals in the public sector are more likely to be risk averse than individuals in the 
private sector. Buurman (2012) found "clear support for the hypothesis that public sector employees are more risk averse than private sector employees" (281). This could provide one possible explanation for the empirical observations in this study.

What the literature appears to suggest is that preferences for risk embed within the strategic environment that the special district inhabits (Bellante and Link 1981; Buurman 2012; Micheli et al. 2012). The strategic environment represents the totality of circumstances, conditions, and influences in which the organization embeds. It describes an amalgamation of the socio-economic and socio-political factors that constitute the political economy (Porter 1990; Morgan 2006).

Various conditions and factors combine in the dynamic milieu that is the strategic environment to affect the organization and the leader's decision-making processes in often-unpredictable ways. While risk preferences are not fixed or immutable, this dissertation revealed that there are distinct institutional and individual preferences. In this regard, structural factors surrounding the co-production of public sector innovation hold the key to defining risk aversion as it relates to the co-production of public sector innovation (Micheli et al. 2012).

The first conclusion of this finding is that the antecedent of risk-taking preferences is neither necessary nor sufficient for the co-creation of public sector innovation. In this regard, risk preferences do not open the pathway to the co-production of public sector innovation. In other words, the effect of risk preferences is irrelevant to the co-production of public sector innovation. This antecedent is not capable of explaining or predicting the presence or absence of the phenomenon. This is the case since low levels of individual preferences for risk exist independent of the manifestation 
of the phenomenon. The first conclusion leads to the recommendation to modify the model to eliminate the antecedent of risk-taking preferences.

One aspect of risk highlighted in the cases is the multifaceted nature of the phenomenon. In other words, the empirical observations revealed individual perceptions that risk existed in any number of manifestations. The two primary manifestations of the phenomenon of risk were physical and psychological in nature. Physical risk represented an exposure of individuals or property to potential physical harm, damage, or loss. The special districts mitigated these types of risk, for example, through insurance and workplace safety programs. Psychological risk, on the other hand, represents exposure to potential harm or loss that results from mental anguish, frustration, or intellectual distress. Efforts to mitigate psychological risk involved communication, feedback loops, and the exercise of transformational leadership.

The second conclusion is that the independent variable of individual risk-taking preference is neither a necessary nor a sufficient measurement. As an extraneous independent variable, it represents something other than an independent variable that might alter the outcome of the case study. In this regard, while it may serve to indicate the presence of the antecedent of risk-taking preferences this antecedent does not create a pathway to the co-production of public sector innovation. Since the antecedent of risktaking preferences is irrelevant, the independent variable that measures this antecedent is irrelevant as well within the context of this model. This conclusion leads to the recommendation to modify the model to eliminate the independent variable labeled individual risk-taking preferences. 


\section{Summary of Findings and the Revision of the Model}

Table 21 depicts the revised organizational antecedents of public sector innovation. This table includes the independent variables that served to indicate the presence of the antecedents. The table also indicates the domains in which the antecedents were operative. The utility and efficacy of the changes was borne out in the empirical observation of the presence of the dependent variable of co-produced innovation in the public sector when the independent variables in table 21 were present. The previous section of this chapter explained the reasoning behind the changes, and the nature of these modifications in detail.

Table 21: Revised Organizational Antecedents of Public Sector Innovation

\begin{tabular}{|l|l|l|l|l|l|}
\hline \multicolumn{2}{|c|}{ Domain } & \multicolumn{2}{|c|}{ Antecedent } & \multicolumn{2}{|c|}{ Independent Variable } \\
\hline \hline 1 & $\begin{array}{l}\text { Leader-Client } \\
\text { Co-Production }\end{array}$ & 1.1 & $\begin{array}{l}\text { Adaptive Interest } \\
\text { Alignment }\end{array}$ & 1.1 .1 & Existence of feedback loops \\
\hline 2 & $\begin{array}{l}\text { Client- } \\
\text { Organization } \\
\text { Co-Production }\end{array}$ & 2.1 & $\begin{array}{l}\text { Client-Based } \\
\text { Prioritization }\end{array}$ & 2.1 .1 & Existence of resource constraints \\
\cline { 3 - 6 } & 2.2 & $\begin{array}{l}\text { Co-Production } \\
\text { Readiness }\end{array}$ & 2.2 .1 & Co-production gap awareness \\
\hline 3 & $\begin{array}{l}\text { Organizational- } \\
\text { Leader Co- } \\
\text { Production }\end{array}$ & 3.1 & $\begin{array}{l}\text { Organizational } \\
\text { Structure/Culture }\end{array}$ & 3.1 .1 & Transformational leadership \\
\cline { 3 - 6 } & 3.2 & $\begin{array}{l}\text { Organizational } \\
\text { Incentives }\end{array}$ & 3.2 .1 & $\begin{array}{l}\text { Intrinsic incentives for } \\
\text { improvement activity }\end{array}$ \\
\hline
\end{tabular}

Table 21 reflects the elimination of four independent variables: leading with a vision, sunsetting through co-production, ability to experiment, and individual risk-taking preferences. The table also reflects the modification of the independent variable incentives for improvement activity. The table reflects the elimination of the antecedent risk-taking preferences. Conditions of necessity and sufficiency led to the elimination of 
three of the independent variables. In other words, three of the variables were neither necessary nor sufficient for the emergence of the dependent variable. The modification of the one independent variable served to improve measurement accuracy. The elimination of the risk-taking preferences and its associated independent variable were the result of extraneousness.

The revisions to the model have the benefit of balancing the antecedents across the domains. These modifications have the effect of reducing the number of independent variables to one or two per antecedent. There are now, likewise, between one and two antecedents operative within each of the three domains. The modifications result in a more nuanced integration of the antecedents within their respective domains. The changes have the effect of providing a more parsimonious model. These adjustments provide a more robust empirical framework for explaining and predicting the cause of coproduced public sector innovation.

The revised model does not contain the independent variable of leading with a vision. As noted earlier, this was not an adequate indicator of the presence of the antecedent of adaptive interest alignment. Instead, leading with a vision is one of the traits that serves as a measure of the independent variable transformational leadership. There was, likewise, the elimination of the independent variable of sunset through coproduction as an indicator of the presence of the antecedent co-production readiness. This is the case since sunset through co-production is neither a necessary nor a sufficient condition for the co-production of public sector innovation. As such, it is not an adequate indicator of the antecedent of co-production readiness. 
The third independent variable to face elimination from the original model is the ability to experiment. This is the case, since the ability to experiment is neither a necessary, nor a sufficient, condition for an innovative organizational structure and culture. It is, therefore, not an adequate indicator of the antecedent of organizational structure and culture. The final independent variable to face elimination was individual risk-taking preference. Unlike the other three independent variables — whose elimination was the result of a lack of necessity and sufficiency - the independent variable of individual risk-taking preferences was extraneous. This was the case because the antecedent of risk-taking preference was extraneous.

Table 21 depicts the revised theoretical framework in which the independent variable incentives for improvement now reflects only intrinsic incentives and excludes extrinsic incentives. This modification was necessary to ensure the model properly reflected a true measure of this independent variable. In this regard, extrinsic incentives are neither a necessary nor a sufficient organizational incentive to innovate. Conversely, intrinsic incentives are a necessary, but not sufficient, condition for the antecedent of organizational incentives to open the pathway to the co-production of public sector innovation.

Figure 12 depicts the revised strategic dimensions of public sector organizational innovation model. The changes reflect modifications made to the theoretical framework that incorporated the findings of this dissertation. The alterations reflected a further refinement of the model based on empirical observation and analysis. The adjustments served to improve the granularity and add clarity to help understand the causal mechanisms the model seeks to explain. The core domains operative in the model remain 
unchanged. The finding related to the phenomenon of risk was the most significant in this study.

Figure 12. Revised Strategic Dimensions of Public Sector Organizational Innovation Model

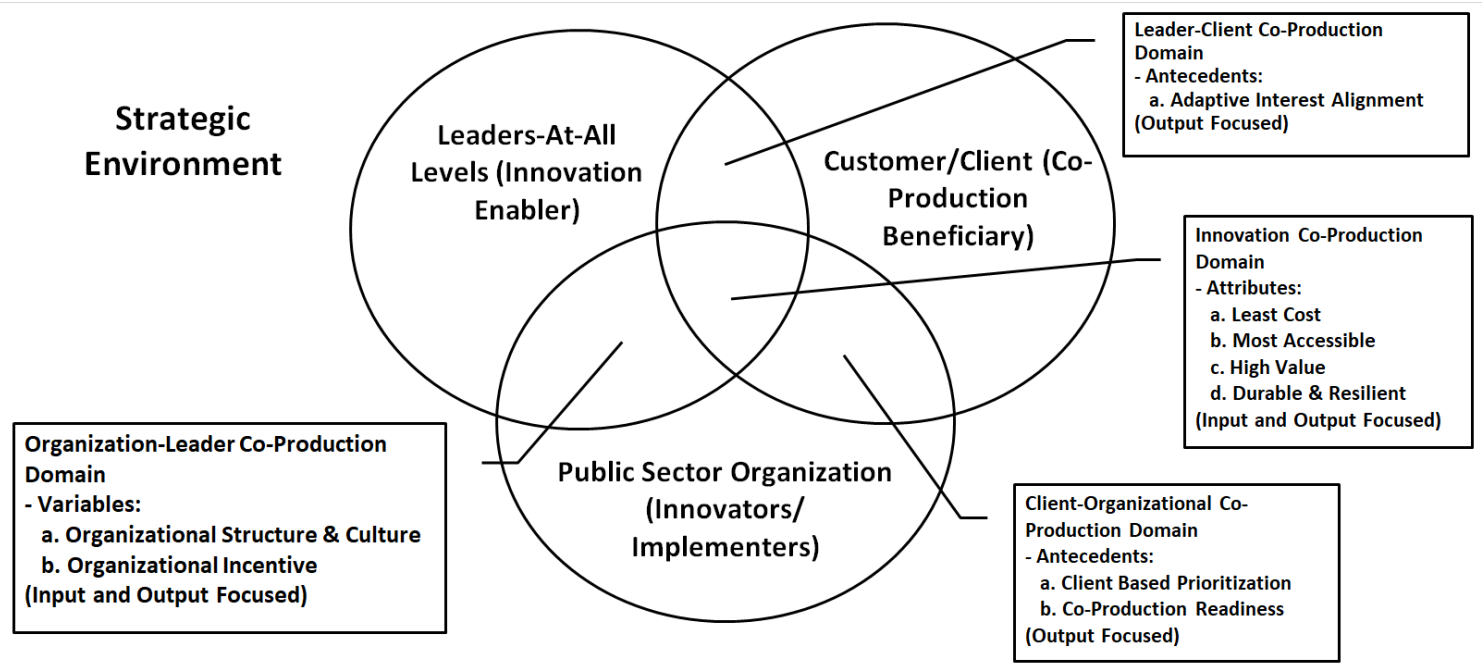

Because of the conclusions and recommendations related to this phenomenon, the number of antecedents narrowed to five: adaptive interest alignment, client-based prioritization, co-production readiness, organizational incentives, and organizational structure and culture. All but one of these five antecedents have a single independent variable attached to them. This was not the case previously, where several antecedents had multiple independent variables.

\section{Value of this Study to Practice, Theory, and Policy}

Theory represents an exercise in simplifying social reality (Dubin 1969). The theoretical framework validated in this dissertation parsimoniously achieves two aims. 
The first is to understand the social interactions that result in the phenomenon of coproduced public sector innovation. This dissertation accomplished this by providing new knowledge and insight into the antecedents that create the pathway to the co-production of public sector innovation. The second aim this study achieved was to provide a model that is capable of predicting the emergence of this phenomenon. In this regard, the model anticipates the phenomenon through the indexed measurement of discrete independent variables.

Dubin (1969) asserts that, "The generality of a scientific model depends solely upon the size of the domain it represents" (141). Dubin (1969) is referring to a different definition of domain than that which the model specifies. He defines "the domain of the model as being the territory over which [individuals] can make truth statements about the model and, therefore, about the values of the units composing the model" (Dubin 1969, 137-138). The term “isomorphic institutional form” will serve in place of Dubin's (1969) "domain" to avoid confusion, and to more accurately define the domain within the context of this study. In the context of this study, the general isomorphic institutional form studied is the public sector organization.

The institutional forms analyzed in this study have specific boundaries. These boundaries, while amorphous, serve to define that portion of the empirical world the theoretic framework is modeling. Dubin (1969) observes that, "The boundarydetermining criteria of a model apply with equal force to the units employed and the laws of interaction among these units" (128). The nominal boundary criterion of this study are special districts. The question of generalizability is dependent on the degree to which public sector organizations are essentially the same or similar to special districts. The 
boundary of this model exceeds "when either a new unit or a new law or both are required to make the model work" (135). Dubin (1969) refers to this as the point of discontinuity where "either the units or the laws or both need to be changed in order to model the phenomenon being studied" (134).

To the extent organizations share the same boundary criteria, the level of risk associated with generalizing the theoretical framework to these organizational and institutional types is low. In other words, it is possible to generalize the findings, conclusions, and recommendations beyond the public maritime ports in the states of Oregon and Washington. This is the case only in instances where the organizations share the same boundary conditions. There is, however, a level of risk associated with pursuing generalization if the institutional forms are not isomorphic. The degree of isomorphism plays a critical role in defining the risk of generalization.

The extent to which it is possible to generalize the model is dependent on a number of institutional factors that serve as the conditions, principles, measures for establishing the boundary criteria. The isomorphic institutional form to which the model applies is strictly bounded (Dubin 1969). The research of Morgan et al. (2013) into the relationships between special district governments, general-purpose governments, nonprofit organizations, and for-profit private agencies provides a prism for viewing the issue of determining boundary criteria.

Morgan et al. (2013) propose a two-degree model of institutional isomorphism. The scope of the interests and the manner of value arbitration define the boundary criteria (Morgan et al. 2013). According to Morgan et al. (2013), values are either parochial or common. This is their first essential difference between institutional forms. Parochial 
interests are those that a narrow group shares, where benefits of the governmental entity accrue to a limited few. Common interests, conversely, are those shared by society. There achievement of common interests benefits a many people. The arbitration of values is through market mechanisms or political processes (Morgan et al. 2013). This is the second essential difference. Market mechanisms reflect the interplay of supply and demand in determining the prices, quantity, and quality of goods and services. These benefits are available to individuals in society through a nominally free market. Political processes are dependent on public governance to create goods and services.

Dubin (1969) asserts that, "The domain of the model is that portion of the empirical world included within the boundaries" of the model (145). I assert that the theoretical framework advanced in this dissertation both explains and predicts the coproduction of public sector innovation in US ports. There is a relatively low risk in making this generalization since US ports are largely isomorphic with respect to one other. It is possible, therefore, to extend the research findings, conclusions, and recommendations of the sample population to the larger population of US ports.

Another low-risk generalization is to extend the theoretical framework to other types of special districts or special-purpose governments. This is the case since these organizations are isomorphic institutional forms as well. They share the same degrees of institutional form. Special districts all have in common the political arbitration of parochial interests. This suggests a high degree of institutional isomorphism is present between the study sample and the larger population of special districts (Morgan et al. 2013). As such, they fall within the boundaries of the model. There is, therefore, a relatively low risk in making this generalization. 
The generalizability of the framework advanced in this dissertation to generalpurpose governments and for-profit private agencies and firms in the public sector is more problematic. Each institutional form has one essential difference when compared to special district governments (Morgan et al. 2013). For general-purpose governments, this is the scope of interests- parochial or common. For for-profit private agencies and firms, this is the manner of value arbitration—market mechanisms or political processes.

There is a likelihood that sharing a single essential similarity is sufficient to generalize this model to these institutional forms. The same academic disciplines encompass special-purpose governments, general-purpose governments, and for-profit private agencies and firms. Each of these institutional forms share common boundaries when it comes to organizational theory (Salamon 2003; Scott 2003; Morgan 2006). It is, therefore, probable that the framework is capable of explaining and predicting the coproduction of public sector innovation in general-purpose governments and for-profit private agencies. Future research is necessary, however, to confirm this possibility.

The generalizability of this model to public sector non-profit organizations is even more risky. This is the case because these institutional forms are different in scope of interests and manner of value arbitration from special districts (Morgan et al. 2013). Public sector non-profit organizations share academic disciplines with the public and private sector and have some common boundaries when it comes to organizational theory (Salamon 2003). There is a consensus in the literature that organizations in the nonprofit sector are isomorphic with respect to other institutional forms in the public sector (Morgan 2006; Scott 2003). It is, however, unknown if the model will fully or adequately 
explain or predict innovation in the non-profit sector. Future research is necessary in this area.

There is a high level of risk associated in generalizing the model to overseas ports or foreign public sector government organizations. This includes extending the findings, conclusions, and recommendations to overseas ports or foreign public institutions. This is the case because co-production of public sector innovation is contextual in nature. I suspect that differing cultural norms and values will render this culturally specific model invalid. Likewise, differing institutional forms of similar organizations could invalidate the model. Future research is necessary to confirm if this is the case.

The theoretic framework has utility for theorists, practitioners, and policymakers in the public sector. Each of these groups approaches the co-production of public sector innovation in a different manner. What they all share in common, however, is a focus on solving social problems. The theorist, for example, is interested in developing and testing theoretical constructs that enhance the understanding of human interactions and the ability to predict social outcomes (Dubin 1969). The practitioner, on the other hand, requires a heuristic set of tools in order to create the conditions for public sector innovation to emerge (Micheli 2012). The policymaker, finally, seeks to define prescriptions for public action that achieves a social need (Dunn 2012).

The recommendations contained in this chapter typically fell into one of two categories. The first category was recommendations to adjust or modify the model. The empirical observations served as the basis for these recommendations. This set of recommendations reflected enhancements to improve the fidelity of the model. The revision of the model based on the empirical evidence was the subject of the previous 
section of this chapter. The theoretic framework presented in this study has utility for theoreticians and academics researching public sector innovation. They now have an empirically validated model of the co-production of public sector innovation to test and evaluate.

This dissertation represents an exercise in theory building that was both deductive and inductive. The prior work of several theoreticians and academic researchers informed the theoretic framework. Deductively building on past work allowed for the advancement of the study of public sector innovation. Putting the model at risk by subjecting it to rigorous empirical observation ensured its inductive validity. This model is valid to the extent it accurately describes and predicts social outcomes given a bounded set of conditions. Future research is necessary to rigorously test the model. This testing will further validate the model and allow for the development of confidence in the generalizability of the framework.

The model provides public sector organizations a heuristic framework for fostering the co-production of public sector innovation. At a time when governments are simultaneously facing austere budgets and at the same time raising demands for public goods and services, this model provides a potential source of relief through innovation (Micheli et al. 2012). Facing these twin challenges, the model provides tangible guidance on what works when seeking to achieve public aims through innovation. In addition to the twin challenges of providing more with less, wicked challenges are not easily amenable to quick or easy solutions. The model has utility in seeking to solve wicked challenges as well through innovation. 
The second purpose of these recommendations was to support practitioners in the field of public administration and public policy by empowering public sector leaders in their efforts to co-produce public sector innovation. These recommendations represented an attempt to make the model accessible for everyday use in the public sector. The recommendations are practical and empirically verified. They serve to create the conditions that lead to the emergence of the antecedents that create the pathway to the coproduction of public sector innovation.

When executing the recommendations, the theoretic framework provides guidance on measuring the success of this effort as well. In this regard, it is possible to measure if the recommendations are successful in eliciting public sector innovation. This is possible by measuring the independent variable associated with the antecedent. A high measure, for example, indicates a successful effort. In other words, a high measure of the independent variable indicates the presence of the antecedent.

Policymaking involves dealing with a range of theoretical and practical policy issues at the local, state, regional, national, and international levels (Dunn 2012). While the themes for public sector innovation and special districts dominate this dissertation, the essential essence of this study is policy-making within the context of pubic governance. Good public governance, moreover, is only possible through an integrated framework of political, administrative, and policy processes. Public sector innovation represents a multi-disciplined approach to creating the socioeconomic and institutional frameworks within which it is possible to formulate, implement, administer, and evaluate public policy. Public sector innovation provides the mechanism thought which policymakers address complex social issues. 


\section{Future Research Opportunities}

What follows in this section is an exploration of future research opportunities. Three themes animate this section. The first is the need to meticulously test and refine the model. This dissertation represents an exercise in theory building using a case study approach to validate the model. Rigorous testing of the model, therefore, is still required. The second theme is the need to address potential limitations of the study. Each methodological approach to theory building and testing has strengths and weaknesses. Understanding the potential impact of these limitations on the results is necessary to put the theoretic framework in perspective. The third, and final, theme this section touches upon is the need to expand the boundaries of the model. Increasing the margins of the model serves to support the generalizability of the theoretic framework.

Regarding the first theme, replication of this study is desirable for a few reasons. The first reason is to test the model. Only through rigorous testing of the model is it possible to ensure the theoretic framework represents an accurate display of the social world (Dubin 1969). There is a difference between validating a theoretical model and proving or testing a theoretical model (Little 1991). The former is the subject of this dissertation, and the latter is outside its scope. Validation of a theoretic framework involves the process of verifying the accuracy and credibility of the model in explaining a social phenomenon (Popper 1959). Testing a theory is the deliberate attempt to falsify or refute the model's explanation of a social phenomenon (Popper 1959).

Testing also serves to refine the framework. The objective of any model is to provide a parsimonious explanation of social reality within the most expansive 
boundaries. Within these borders, there should exist a rich tapestry of social interactions tied to the phenomenon. Table 22 identifies some of these social connections and details the emergent themes or topical insights that manifest within this study. These themes or insights arose during the study of the cases. They are the result of the empirical observations and emerged during the analysis of the data. The themes or insights provide a number of jumping off points for the future study of the co-production of innovation in the public sector. As such, they represent promising avenues of future research. They also provide opportunities to build on this research to expand our understanding of the phenomenon.

The second theme for future research deals with addressing potential limitations of the study. Successful research into social phenomenon is dependent upon the selection and application of a methodological approach that meets the aims of the study. I argue for the use of methodological pluralism to study the co-production of public sector innovation (Little 1991). Each methodological approach, moreover, has unique strengths and weaknesses. This means that future research has the potential of compensating for any limitations inherent in this study's methodological approach. There is, for example, the danger that the qualitative study of one case or a small assortment of cases is particularistic in nature. This prevents generalizability beyond the sample.

It is possible to address the issue of particularism with a quantitatively structured approach. Little (1991) asserts that, "Statistical correlation is explanatory to the extent that it provides a credible causal process underlying the variables being analyzed" (159, emphasis in original). It is possible, therefore, to study a subset or sample of a population to draw inferences on the whole of the population (Kumar 2011). Statistical tools are, 


\section{Table 22. Emergent Themes or Topical Insights}

\begin{tabular}{|c|c|c|}
\hline Antecedent & Measurement & Theme or Insight (Case \#) \\
\hline \multirow{5}{*}{$\begin{array}{l}\text { Adaptive } \\
\text { Interest } \\
\text { Alignment }\end{array}$} & \multirow{4}{*}{$\begin{array}{l}\text { Existence of } \\
\text { feedback loops }\end{array}$} & Inherent complexity of feedback loops (1) \\
\hline & & $\begin{array}{l}\text { Interests adapt and align around the normative value of the } \\
\text { communities in which the special district embeds (2) }\end{array}$ \\
\hline & & $\begin{array}{l}\text { Ad Hoc and serendipitous nature of informal feedback loops } \\
\text { (1) }\end{array}$ \\
\hline & & Feedback loops amplify interest group demand signals (3) \\
\hline & Lead with vision & Creativity and innovation are intrinsically linked (1) (2) \\
\hline \multirow{5}{*}{$\begin{array}{l}\text { Client-Based } \\
\text { Prioritization }\end{array}$} & \multirow{4}{*}{$\begin{array}{l}\text { Existence of } \\
\text { resource } \\
\text { constraints }\end{array}$} & Innovation begets innovation (1) \\
\hline & & Serendipity plays a role in fostering innovation (1) \\
\hline & & Special districts often chase their own tails (2) \\
\hline & & $\begin{array}{l}\text { Sensitivity to the existence and effect of socio-economic } \\
\text { divisions in communities in which they reside (2) }\end{array}$ \\
\hline & $\begin{array}{l}\text { Co-production } \\
\text { gap awareness }\end{array}$ & $\begin{array}{l}\text { Existence of co-production awareness gap results in partner } \\
\text { negotiation (1) (3) }\end{array}$ \\
\hline \multirow[t]{2}{*}{$\begin{array}{l}\text { Co-Production } \\
\text { Readiness }\end{array}$} & $\begin{array}{l}\text { Sunset through } \\
\text { co-production }\end{array}$ & $\begin{array}{l}\text { Path dependencies play an important role in innovation (1) } \\
\text { (2) }\end{array}$ \\
\hline & $\begin{array}{l}\text { Information } \\
\text { symmetry }\end{array}$ & $\begin{array}{l}\text { Information symmetry was sought at project/program } \\
\text { decision points for phasing in/out (1) }\end{array}$ \\
\hline \multirow{4}{*}{$\begin{array}{l}\text { Organizational } \\
\text { Structure/ } \\
\text { Culture }\end{array}$} & \multirow{2}{*}{$\begin{array}{l}\text { Transformational } \\
\text { leadership }\end{array}$} & Culture of trust is necessary to foster innovation (1) \\
\hline & & Leaders foster a sense of community around innovation (1) \\
\hline & \multirow[t]{2}{*}{$\begin{array}{l}\text { Ability to } \\
\text { experiment }\end{array}$} & $\begin{array}{l}\text { Perception normative vales (public trust and stewardship) } \\
\text { could conflict with practice of innovation (2) }\end{array}$ \\
\hline & & $\begin{array}{l}\text { Level of systematic experimentation is an artifact of } \\
\text { organizational size/resource availability (2) }\end{array}$ \\
\hline \multirow{3}{*}{$\begin{array}{l}\text { Organizational } \\
\text { Incentives }\end{array}$} & \multirow{3}{*}{$\begin{array}{l}\text { Incentives for } \\
\text { improvement } \\
\text { activity }\end{array}$} & Creating a culture of sharing credit around innovation (1) \\
\hline & & Organizational culture provides incentives for innovation (1) \\
\hline & & $\begin{array}{l}\text { Gap exists between the perception of the intended an actual } \\
\text { level of intrinsic reward (1) (3) }\end{array}$ \\
\hline \multirow{5}{*}{$\begin{array}{l}\text { Risk-taking } \\
\text { Preferences }\end{array}$} & \multirow{5}{*}{$\begin{array}{l}\text { Individual risk- } \\
\text { taking } \\
\text { preference }\end{array}$} & Risk is ubiquitous and requires careful mitigation (1) \\
\hline & & Risk and its mitigation take a number of forms (1) (2) \\
\hline & & Perception of risk is temporal in nature (1) (2) \\
\hline & & $\begin{array}{l}\text { Risk preferences vary depending on circumstances and } \\
\text { conditions in the strategic environment (1) (2) }\end{array}$ \\
\hline & & Risk perception is multifaceted (2) \\
\hline
\end{tabular}


therefore, capable of empirically evaluating causal relationships, although statistical regularities are not themselves explanatory (Little 1991). Qualitative methodologies and statistical correlation, as such, can serve as the basis for empirically testing a theory (Little 1991).

This dissertation makes no claims to the sustainability or diffusion of innovation. This is a potential weakness of the study. Conducting a longitudinal study of the cases would support the determination of the sustainability of the co-produced innovations (Kumar 2011). A longitudinal study is, however, beyond the scope of the present study. While this dissertation touched upon the issue of quality, it was as an attribute of the independent variables and not of the dependent variable. Creating an index to measure the quality or robust nature of co-produced public sector innovation would also support an understanding of the sustainability and diffusion of innovation.

It is possible to use various research methodologies to view the phenomenon from differing perspectives. Future research into this phenomenon should vary the research design. A survey design, for example, could increase the quantitative profile of this research. Differing approaches could include future research that adjusts the method of data collection to include, for example, questionnaires. Follow-on research could also modify the questions in an attempt to gain greater fidelity of the dependent variable. It is also possible to vary the reference period and inject experimental components into the research design (Kumar 2011).

Regarding the third theme for future research, it is necessary to create theoretical and empirical boundaries in any research endeavor (Dubin 1969). Dubin (1969) asserts that, "In order that a model may represent an empirical system, it has to have boundaries 
corresponding to the empirical system" (126). In addition to testing the model and understanding the limitations of the model, future research can assess these boundaries. Further study is necessary, for example, to definitively determine if it is possible to generalize the model to other institutional forms in the public sector.

There is some risk in applying this model to general-purpose governments or forprofit private agencies and firms for the reasons outlined in the previous section. Empirical testing is necessary to determine with a high level of confidence that this study's findings are generalizable beyond public maritime ports in the Pacific Northwest. While this is probably the case, analysis of the model in these empirical settings is required to confirm this assertion. The same is true with respect to non-profit organizations. Future investigations hold the promise of expanding the boundaries to include these organizational types. 


\section{REFERENCES}

Acher, Wourer van, and Geert Bouckaert. 2017. "What makes public sector innovation survive? An exploratory study of the influence of feedback, accountability and learning." International Review of Administrative Sciences 0 (0): 1-20.

Adams, Richard, John Bessant and Robert Phelps. 2006. "Innovation management measurement: A review." International Journal of Management Reviews (n.d.): $21-47$.

Aguilar, Francis Joseph. 1988. General managers in action. New York: Oxford University Press.

Albury, David. 2005. "Fostering innovation in public services." Public Money and Management (January): 51-56.

Alford, John. 1998. "A public management road less traveled: Clients as co-producers of public services." Australian Journal of Public Administration 57 (4): 128-37.

Almond, Gabriel, and Stephen Genco. 1977. "Clouds, clocks, and the study of politics." World Politics 29 (July): 489-522

American Association of Public Ports (AAPA). 2012. Seaports of the America's annual directory. Alexandria, VA: AAPA

Arundel, Anthony, and Hugo Hollanders. 2011. "A taxonomy of innovation: How do public sector agencies innovate?" 2010 European Innobarometer Survey of Public Agencies Report. Maastricht: University of Maastricht.

Australian Public Service Commission (APSC). 2011. Measuring innovation in the public sector: A literature review, draft report. Canberra: Commonwealth of Australia.

Bason, Christian. 2010. Leading public sector innovation. Bristol, UK: The Policy Press.

Bass, Bernard M. 1985. Leadership and performance beyond expectations. New York: Free Press

Bauroth, Nicholas G. 2010. "The strange case of the disappearing special districts: Toward a theory of dissolution." The American Review of Public Administration 40.5, 568-592.

Bellante, Don, and A.N. Link. 1981. "Are public sector workers more risk averse than private sector workers?” Industrial and Labor Relations Review 34 (April): 
408-412.

Besley, Timothy, and Maitreesh Ghatak. 2005. "Competition and incentives with motivated agents.” American Economic Review 95 (3): 616-636.

Bessant, John. 2005. "Enabling continuous and discontinuous innovation: Learning from the private sector." Public Money and Management (January): 35-42.

Bessant, John, Tim Hughes and Sue Richards. N.d. "Beyond light bulbs and pipelines: Leading and nurturing innovation in the public sector." n.d. http://www.mindlab.dk/assets/399/BeyondLightbulbs.pdf (May 3, 1014).

Bessant, Joseph R., and Keith Pavitt. 1997. Managing innovation: Integrating technological market and organizational change, $2^{\text {nd }}$ ed. New York: John Wiley and Sons.

Birkinshaw, Julian, Gary Hamel, and Michael J. Mol. 2008. "Management innovation." Academy of Management Review 33 (October): 825-845

Bloch, Carter, and Markus M. Bugge. 2016. "Public sector innovation-from theory to measurement." Public Money and Management 36 (4): 281-288.

Bock, Edwin A. 1977. "Chapter 21, Improving the usefulnesses of the case study." Donald M. Freeman, ed. Foundations in Political Science. New York: Free Press, 674-697.

Bollens, Scot A. 1986. "Examining the link between state policy and the creation of local special districts." State and Local Government Review, 18.3, 117-124.

Booth, Wayne C., Gregory G. Colomb, and Joseph M. Williams. 2008. The craft of research, $3^{\text {rd }}$ ed. Chicago, IL: The University of Chicago Press.

Borins, Sandford. 2001. "Encouraging innovation in the public sector." Journal of Intellectual capital (n.d.): 310-319.

Borins, Sandford. 2000. "What border? Public management innovation in the United States and Canada." Journal of Policy Analysis and Management (Winter): 46-74.

Borins, Sandford, and Richard M. Walker. 2012. "Many are called but few are chosen: Modeling the selection process for the innovations in American government awards." December http://www.innovations.harvard.edu/showdoc.html?id=2638028 (May 26, 2014).

Bovaird, Tony. 2007. "Beyond engagement and participation: User and community coproduction of public services." Public Administration Review 67 (5): 846-860. 
Boyle, David, and Michael Harris. 2009. “The challenge of co-production.” London: Economics Foundation.

Brudney, Jeffrey L., and Robert E. England. 1983. "Toward a definition of the coproduction concept." Public Administration Review 43 (1): 59-65.

Bugge, Markus, and Carter Bloch. 2013. "Between bricolage and breakthroughs -framing the many faces of public sector innovation." Structural Change and Economic Dynamics 27 (July): 133-145.

Bundy, Wayne Milers. 2002. Innovation, creativity, and discovery in modern organizations. Westport, CT: Quorum Books.

Burns, James MacGregor. 1978. Leadership. New York: Harper and Row.

Burns, Nancy. 1994. The formation of American local governments: Private values in public institutions. New York: Oxford University Press.

Burns, Thom, and G.M. Stalker. 1994. The management of innovation, Rev. ed. Oxford: Oxford University Press.

Burpitt, William J., and William J. Bigoness. 1997. "Leadership and innovation among teams: the impact of empowerment." Small Group Research (n.d.): 414-423.

Buurman, Margaretha, Josse Delfgaauw, Robert Dur, and Seth Van den Bossche. 2012. "Public sector employees: Risk adverse or altruistic." Journal of Economic Behavior and Organization 83 (n.d.): 279-291.

Camisón-Zornoza, César, Rafael Lapiedra-Alcamí, Mercedes Segarra-Ciprés and Montserrat Boronat-Navarro. 2004. "A meta-analysis of innovation and organizational size.” Organization Studies (n.d.): 331-361.

Carr, Jered B. 2006. "Local government autonomy and state reliance on special district governments: A Reassessment." 59.3, 481-492

Chen, Chung-An, Evan M. Berman, Jonathan P. West, and Robert J. Eger III. 2013. "Community commitment in special districts." International Public Management Journal. 16, 113-140

Cohen, Michael, James G. March, and Johan P. Olsen. 1972. “A garbage can model of organizational choice.” Administrative Science Quarterly 17 (March): 1-25. JSTOR (March 24, 2012).

Collins, Jim. 2005. "Level 5 leadership: the triumph of humility and fierce resolve." 
Harvard Business Review (July): 136-149.

Cooper, Phillip J. 2003. Governing by contract. Washington, D.C.: CQ Press.

Cunningham, Paul, and Aikaterini Karakasidou. 2009.'Innovation in the public sector." Polity Brief N2. Manchester, UK: University of Manchester.

Dahl, Robert A. 1956. A preface to democratic theory. Chicago: University of Chicago Press.

Damanpour, Fariborz. 1987. "The adoption of technological, administrative, and ancillary innovations: Impact of organizational factors." Journal of management (n.d.): 675-688.

Damanpour, Fariborz. 1987. "The adoption of technological, administrative, and ancillary innovations: Impact of organizational factors." Journal of management (n.d.): $675-688$

Damanpour, Fariborz. 1992. "Organizational size and innovation." Organization Studies 13 (n.d.): 375 - 402

Davis, Christopher R., and Marcus Ingle. 2013. "Bridging EMERGE leadership and breakthrough innovation in public service: assessment of compatibility and sufficiency, and a new EMERGE tool." Presented at the Third International Conference on Government Performance, Tokyo.

Delfgaauw, Josse, and Robert Dur. 2010. "Managerial talent, motivation, and selfSelection into public management." Journal of Public Economics 94 (June): 654-660.

Deming, W. Edwards. 1986. Out of the crisis. Cambridge, MA: MIT

Denhardt, Rober B., Janet Denhardt, and Maria P. Aristigueta. Managing human behavior in public and private organizations, $3^{\text {rd }}$ ed. Los Angeles: Sage.

DiMaggio, Paul J., and Walter W. Powell. 1983. "The iron cage revisited: institutional isomorphism and collective rationality in organizational fields." American Sociological Review 48 (April): 147-160.

Downs, Anthony. 1957. An economic theory of democracy. Boston: Addison Wesley.

Dror, Yehezkel. 2001. The capacity to govern. New York: Routledge.

Drucker, Peter F. 1998. "The discipline of innovation." Harvard Business Review (November-December): 1-8. 
Dubin, Robert. 1969. Theory building. New York: Free Press.

Dunn, William N. 2012. Pubic policy analysis. $5^{\text {th }}$ ed. Boston: Pearson.

Edquist, Charles. 2001. "Innovation policy: A systematic approach.” Daniele Archibugi and Bengt-Åke Lundvall, ed. The globalizing learning economy. Oxford: Oxford University Press, 219-37.

Elenkov, Detelin S., William Judge and Peter Wright. 2005. "Strategic leadership and executive innovation influence: An international multi-cluster comparative study." Strategic Management Journal (July): 665-682.

Ericsson, K. Anders, Michael J. Prietula, and Edward T. Cokely. 2007. "The making of an expert." Harvard Business Review (July): 114-121.

Ettlie, John E., William P. Bridges and Robert D. O'Keefe. 1984. “Organization strategy and structural differences for radical versus incremental innovation." Management Science (June): 682-695.

European Commission (EU). 2013. European public sector innovation scoreboard 2013: A pilot exercise." Brussels: European Commission.

Farrington, John, and Conor Farrington. 2005. "Rural accessibility, social inclusion and social justice: towards conceptualization." Journal of Transport Geography 13 (n.d.): 1-12.

Ferris, James M. 1984. "Coprovision: Citizen time and money donations in public service provision." Public Administration Review 44 (4): 324-333.

Foster, Kathryn A. 1997. The political economy of special-purpose government. Washington, D.C.: Georgetown University Press.

Freeman, Christopher. 1987. Technology policy and economic performance: lessons from Japan. London: Pinter.

Freeman, Donald M. 1977. Foundations in Political Science. New York: Free Press.

French, John R. P., and Bertram Raven. 1959. "The basis of power." In Leading for innovation and organizing for results. ed. Frances Hesselbein, Marshall Goldsmith, and Iain Somerville. San Francisco: Jossey-Bass.

Fuglsang, Lars, and Flemming Sørensen. 2011. "The balance between bricolage and innovation: management dilemmas in sustainable public innovation." The Service industries journal (n.d.): 581-595. 
Fukuyama, Francis. 2014. Political order and political decay. New York: Farrar, Straus and Giroux.

Galvan, Sara C. 2007. "Wrestling with MUDS to pin down the truth about special districts." Fordham Law Review 75, 3041-3080

Gates, Scott, and Jeffery Hill. 1995. "Democratic accountability and governmental innovation in the use of nonprofit organizations." Policy Studies Review (February): 137-148.

Gault, Fred. 2010. Innovation strategies for a global economy. Cheltenham, UK: Edward Elgar Publishing, Inc.

Geetz, Clifford. 1971. The interpretation of cultures. New York: Basic Books.

George, Alexander L., and Andrew Bennett. 2005. Case-studies and theory development in the social sciences. Cambridge, MA: MIT Press.

Georges, Gigi, Tim Glynn-Burke, and Andrea McGrath. 2013. Improving the local landscape for innovation part 1: Mechanics, partnerships and clusters. December 4, 2013. Cambridge, MA: Harvard University http://www.innovations.harvard.edu/cache/documents/28494/2849471.pdf.

Georges, Gigi, Tim Glynn-Burke, and Andrea McGrath. 2013. Improving the local landscape for innovation part 2: Framework for an innovative jurisdiction. December 4, 2013. Cambridge, MA: Harvard University http://www.innovations.harvard.edu/cache/documents/28496/2849663.pdf.

Georges, Gigi, Tim Glynn-Burke, and Andrea McGrath. 2013. Improving the local landscape for innovation part 3: Assessment and implementation. December 4, 2013. Cambridge, MA: Harvard University http://www.innovations.harvard.edu/cache/documents/30420/3042090.pdf.

Gersick, Connie J. G. 1991. "Revolutionary change theories: A multilevel exploration of the punctuated equilibrium paradigm." Academy of Management Review 16 (January): 10-36. JSTOR (March 24, 2012).

Gibbs, Jack P. 1972. Sociological theory construction. Hinsdale, IL: Dryden Press

Godenhjelm, Sebastian, and Jan-Erik Johanson. 2017. "The effect of stakeholder Inclusion on public sector project innovation." International Review of Administrative Sciences 0 (0): 1-20.

Goldsmith, Stephen. 2002. "Innovation in government." In Leading for innovation and 
organizing for results. ed. Frances Hesselbein, Marshall Goldsmith, and Iain Somerville. San Francisco: Jossey-Bass.

Goleman, Daniel. 2000. "Leadership that gets results." Harvard Business Review (March): 78-93.

Goleman, Daniel. 2000a. Working with emotional intelligence. New York: Bantam Books

Goleman, Daniel, Richard Boyatzis, and Annie McKee. 2002. Primal leadership: realizing the power of emotional intelligence. Boston: Harvard Business School Press.

Gordon, George J., and Michael E. Milakovich. 1995. Public administration in America. $5^{\text {th }}$ ed. New York: St. Martin's Press.

Gow, James Iain. 2014. "Public sector innovation theory revisited" The Innovation Journal: The Public Sector Innovation Journal 19 (2): 2-22.

Green, Richard T. 2014. "Institutional history and new public governance." In New public governance, ed. Douglas F. Morgan and Briand J. Cook. Armonk, NY: M.E. Sharpe.

Gulick, Luther. 1937. "Notes on the theory of organization." In Classics of organization theory, 7th ed. ed. Jay M.Shafritz, J. Steven Ott, and Yong Suk Jang. New York: Wadsworth.

Gumusluoglu, Lale, and Arzu Ilsev. 2009. "Transformational leadership and organizational innovation: the roles of internal and external support for innovation." The Journal of Product Innovation Management (n.d.): 265-277.

Hage, Jerald. 1994. Formal theory in sociology: Opportunity or pitfall? Albany, NY: State University of New York

Hage, Jerald. 1980. Theories of organization: Form, process, and transformation. New York: Wiley.

Hartley, Jean. 2005. "Innovation in governance and public services: past and present." Public Money and Management (January): 27-34.

Hartley, Jean, Eva Sorensen, and Jacob Torfing. 2013. "Collaborative innovation: A viable alternative to market competition and organizational entrepreneurship." Public Administration Review (November/December): 821-830.

Heclo, Hugh. 2008. On thinking institutionally. Boulder: Paradigm Publishers 
Heifetz, Ronald, Alexander Grashow, and Marty Linsky. 2009. "Leadership in a (permanent) crisis." Harvard Business Review (July-August): 62-69.

Herzberg, Frederick. 1968. “One more time: how do you motivate employees?” Harvard Business Review (January/February): 53-62. Business Sources Premier (March 24, 2012).

Hesselbein, Frances. 2002. Hesselbein on leadership. San Francisco: Jossey-Bass

Holsti, Ole R. 1977. "Chapter 1, From past to present." Donald M Freeman, ed. Foundations in Political Science. New York: Free Press, 278-312.

Hull, Clyde Eiríkur, and Brian H. Lio. 2006. "Innovation in non-profit and for-profit organizations: visionary, strategic, and financial considerations." Journal of Change Management (March): 53-65.

Huntington, Samuel P. 1968. Political order in changing societies. New Haven: Yale University Press.

Ingle, Marcus. 2015. “Organizational innovation readiness tool: beta 2.0.” Presented at the Oregon Innovation Award Kick-off Event and Interactive Seminar, Portland, Oregon, on December 10.

Ingram, Helen, Anne L. Schneider, and Peter Deleon. 2007. "Social construction and policy design." In Theories of policy design, ed. Paul A. Sabatier. Boulder, CO.: Westview Press.

Jansen, Justin J.P., Frans A.J. Van Den Bosch, and Henk W. Volberda. 2006.

"Exploratory innovation, exploitative innovation, and performance: effects of organizational antecedents and environmental moderators." Management Science (November): 1661-1674.

Jaskyte, Kristina, and William W. Dressler. 2005. "Organizational culture and innovation in non-profit human service organizations." Administration in Social Work (n.d.): $23-41$.

Jaskyte, Kristina, and Audrone Kisieliene. 2006. "Organizational innovation: a comparison of nonprofit human-service organizations." International Social Work 49 (n.d.): $165-176$.

John, Peter. 2013. "Experimentation, behavior change and public policy." The Political Quarterly 84 (2): 238-246.

Johnson, Janet Buttolph, and Richard A. Joslyn. 1995. Political science research methods, $3^{\text {rd }}$ ed. Washington, DC: CQ Press. 
Jones, Russell A. 1985. Research methods in the social and behavioral sciences. Sunderland, MA.: Sinauer Associates Inc.

Joshi, Anuradha and Mick Moore. 2003. "Institutionalized co-production: Unorthodox public service delivery in challenging environments." Journal of Development Studies 40 (4): 31-49.

Jung, Dong I., Chee Chowb, and Anne Wu. 2003. "The role of transformational leadership in enhancing organizational innovation: hypotheses and some preliminary findings." The Leadership Quarterly (n.d.) 525-544.

Justice, Jonathan B., and Robert S. Goldsmith. 2006. "Private governments or public policy tools? The law and public policy of New Jersey's special improvement districts.” International Journal of Public Administration. 29, 107-136

Kantur, Deniz and Arzu Iseri-Say. 2012. "Organizational resilience: A conceptual integrative framework.” Journal of Management and Organization 18 (n.d.): 762-773.

Killian, Larita J. 2009. “Three reasons to revisit special districts.” Journal of Government Financial Management. 14-17.

King, Gary, Robert O. Keohane, and Sidney Verba. 1994. Designing social inquiry. Princeton: Princeton University Press.

King, James E. 1948. "The Origin of the Term 'Political Economy." The Journal of Modern History 20 (September): 230-231.

King, Nigel. 1990. "Innovation at work: the research literature.” In Innovation and creativity at work. ed. Michael A. West and James L. Farr. Chichester, UK: John Wiley and Sons.

King, Nigel, and Neil Anderson. 1995. Innovation and change in organizations. London: Routledge.

King, Nigel, and Neil Anderson. 1990. "Innovation and creativity in working groups." In Innovation and creativity at work: psychological and organizational strategies, ed. Michael A. West and James L. Farr. Chichester: UK: John Wiley and Sons.

Kirkpatrick, Evron M. 1977. “Chapter 1, From past to present.” Donald M Freeman, ed. Foundations in Political Science. New York: Free Press, 3-41.

Kohli, Jitiner, and Geoff Mulgan. 2010. Capital ideas: How to generate innovation in the public Sector. Washington DC: Center for American Progress. 
Koivisto, Juha, Pasi Pohjola, and Niina Pitkanen. 2015. "Systemic innovation model translated ion public sector innovation practice." The Innovation Journal: The Public Sector Innovation Journal 20 (1): 1-17.

Kopell, Jonathan G.S. 2001. "Hybrid Organizations and the alignment of interests: The case of Fannie Mae and Freddie Mac." Public Administration Review 61 (4): 468-482.

Kraft, Michael E. and Furlong, Scott, R. 2013. Public Policy: Politics, Analysis, and Alternatives, $3^{\text {rd }}$ ed. Thousand Oaks, CA: Sage.

Kumar, Ranjit. 2011. Research methodology: A step-by-step guide for beginners, $3^{\text {rd }}$ ed. Thousand Oaks, CA: Sage.

Lee, Sang M., Taewon Hwang, and Donghyun Cho. 2012. "Open innovation in the public sector of leading countries." Management Decision 50 (n.d.): 147-162.

Levine, Charles H. and Glenn Fisher. 1984. "Citizenship and service delivery: The promise of coproduction." Public Administration Review 44 (March): 178-189.

Levitt, Barbra, and James G. March. 1988. "Organizational learning." Academy of Management Review (n.d.): 319-340.

Lewis, Jenny M., Lykke Margot Richard, and Erik Hans Klijn. 2017. "How innovation drivers, networking and leadership shape public sector innovation capacity." International Review of Administrative Sciences 0 (0): 1-20.

Light, Paul C. 1998. Sustaining innovation: Creating nonprofit and government organizations that innovate naturally. San Francisco: Josey-Bass Publishers

Lindblom, Charles. 1959. "The science of muddling through." Public Administration Review 29 (Spring): 79-88.

Lipsky, Michael. 1973. "Street level bureaucracy and the analysis of urban reform." In Neighborhood control in the 1970s, ed. George Frederickson. New York: Chandler Publishing Co.

Little, Daniel. 1991. Varieties of social explanation. Boulder, CO: Westview.

Ljungholm, Doina Popescu. 2014. "Transformational leadership behavior in public sector organizations." Contemporary Readings in Law and Social Justice 6 (1): 76-81.

Lundvall, Bengt-Åke. 1988. "Innovation as an interactive process: from user-producer interaction to the national system of innovation." Giovanni Dosi, ed. Technical 
Change and Economic Theory. London: Pinter, 349-69.

Lunenburg, Fred C. 2012. "Organizational structure: Mintzberg's Framework.” International Journal of Scholarly, Academic, Intellectual Diversity 14 (n.d.)

Manin, Bernard, Adam Przeworski, and Susan C. Stokes. 1999. "Introduction.” In Democracy, accountability, and representation, ed. Adam Przeworski, Susan C. Stokes, and Bernard Manin. Cambridge: Cambridge University Press.

March, James G. and Johan Olsen. 2008. "Elaborating the new institutionalism." Oxford handbook of political institutions. Oxford Handbooks Online (September 2009).

Martin, Joanne. 2002. “Organizational cultures: mapping the terrain.” In Classics of organization theory, 7th ed. ed. Jay M.Shafritz, J. Steven Ott, and Yong Suk Jang. New York: Wadsworth.

Maslow, Abraham H. 1943. "A theory of human motivation." Psychological Review 50 (July): 370-396.

McDonald, Robert E. 2007. "An investigation of innovation in nonprofit organizations: The role of organizational mission." Nonprofit and Voluntary Sector Quarterly (June): 256-281.

McGregor, Douglas Murry. 1957. The human side of enterprise. New York: McGrawHill.

Merx-Chermin, Mireille, and Wim J. Nijhof. 2005. "Factors influencing knowledge creation and innovation in an organization." Journal of European Industrial Training (n.d.): 135-147.

Meyer, Alan D. 1985. "Hospital capital budgeting: Fusion of rationality, politics, and economy." Health Care Management Review 10 (n.d.): 17-27.

Meyer, Alan D., and James B. Goes. 1988. "Organizational assimilation of innovations: A multilevel contextual analysis. The Academy of Management Journal (December): 897-923.

Micheli, Pietro, Magnus Schoeman, David Baxter, and Keith Goffin. 2012. "New Business models for public-sector innovation." Research-Technology (September -October): 51-57.

Micklethwait, John, and Andrian Woodridge. 2014. The fourth revolution: the global race to reinvent the state. New York: Penguin Press.

Mills, John Stuart. [1843] 1974. A system of logic, ratiocinative and inductive. Toronto: 
University of Toronto

Mintzberg, Henry. 1983. Structure in fives: designing effective organizations. Englewood Cliffs, NJ.: Prentice-Hall, Inc.

Moore, Michele-Lee, and Frances Westley. 2011. "Surmountable chasms: networks and social innovation for resilient systems" Ecology and Society (March): 5-18.

Morgan, Douglas F., Richard T. Green, Craig W. Shinn, and Kent S. Robinson. 2013. Foundations of public service, $2^{\text {nd }}$ ed. Armonk, NY: M.E. Sharpe.

Morgan, Douglas F., and Craig W. Shinn. 2014. "The foundations of new public governance." In New public governance, ed. Douglas F. Morgan and Briand J. Cook. Armonk, NY: M.E. Sharpe.

Morgan, Gareth. 2006. Images of organizations, Updated ed. Thousand Oaks, CA: Sage Publications, Inc.

Mogren, Eric T. (Rick). 2014. "Agency in networks: Implications for theory and practice.” In New public governance, ed. Douglas F. Morgan and Briand J. Cook. Armonk, NY: M.E. Sharpe.

Myers, Jerome L. Arnold D. Well, and Robert F. Lorch, Jr. 2010. Research design and statistical analysis, $3^{\text {rd }}$ ed. New York: Routledge.

Nankervis, Alan. 2005. Managing services. Melbourne: Cambridge University Press.

Napier, Mark. 2009. "Innovation and leadership in the public sector." Public Sector Innovation Journal (n.d.): 30-32.

Natanson, Maurice. 1977. "Chapter 16, Philosophy and social science: a Phenomenological approach.” Donald M Freeman, ed. Foundations in Political Science. New York: Free Press, 3-41.

Nelson, Richard R., and Douglas Yates. 1978. Innovation and implementation in public organizations. Lexington, MA.: Lexington Books.

Nonaka, Ikujiro, and Hirotaka Takeuchi. 2011. "The wise leader." Harvard Business Review, May, 59-66.

Nooteboom, Bart. 2000. Learning and innovation in organizations and economies. New York: Oxford University Press.

Normann, Richard. 1984. Service management: Strategy and leadership in service businesses. Chichester, UK: Wiley. 
Northouse, Peter Guy. 2004. Leadership: theory and practice. Thousand Oaks: Sage

Nye, Joseph. 2015. Is the American century over. Cambridge, MA: Polity Press.

Nye, Joseph. 2008. The powers to lead. New York: Oxford University Press.

Olson, Mancur. 1971. The logic of collective action. Cambridge: Harvard University Press.

Orazi, Davide Christian, Alex Turrini, and Giovanni Valotti. 2013. "Public sector leadership: New perspectives for research and practice." International Review of Administrative Sciences 79 (3): 486-504.

Osborne, Stephen P. 2006. "Editorial: The new public governance." Public Management Review (8): 377-387.

Osborne, Stephen. 1998. Voluntary organizations and innovation in public services. New York: Routledge.

Osborne, Stephen P., and Kerry Brown. 2005. Managing change and innovation in public service organizations. New York: Routledge.

Osborne, Stephen P., and Louise Brown. 2013. "Introduction: Innovation in public services." In Handbook of innovation in public services, ed. Stephen P. Osborne and Louise Brown. Northampton, MA.: Edward Elgar.

Ostrom, Elinor. 1990. Governing the commons: the evolution of institutions for collective action. Cambridge New York: Cambridge University Press.

Ostrom, Elinor. 1996. "Crossing the great divide: Coproduction, synergy and development." World Development 24 (6): 1073-87.

Ostrom, Elinor, Roy Gardner, and James Walker. 1994. Rules, games, and common-pool resources. Ann Arbor: University of Michigan Press

Oyelaran-Oyeyinka, Banji. 2005. "Systems of innovation and underdevelopment: and institutional perspective.” Discussion Paper Series, no 2005-1. Maastricht: United Nations University.

Parks, Roger B., Paula C. Baker, Larry Kiser, Ronald Oakerson, Elinor Ostrom, Vincent Ostrom, Stephen L. Percy, Martha B. Vandivort, Gordon P. Whitaker, and Rick Wilson. 1981. "Consumers as coproducers of public services: Some economic and Institutional considerations.” Policy Studies Journal 9 (7): 1001-11. 
Patton, Michael Quinn. 2002. Qualitative research and evaluation methods. Thousand Oaks, CA: Sage Publications.

Pennings, Paul, Hans Keman, and Jan Kleinnijenhuis. 1999. Doing research in political science. Thousand Oaks, CA.: Sage Publishing.

Percy, Stephen L. 1984. "Citizen participation in the coproduction of urban services." Urban Affairs Review 19 (4): 431-446.

Perri 6. 1993. "Innovation by nonprofit organizations: Policy and research issues." Nonprofit management and leadership: (n.d.): 397-414.

Perrow, Charles. 1986. Complex organizations: A critical essay, $5^{\text {th }}$ ed. New York: Random House.

Pfeffer, Jeffrey. 1981. "Understanding the role of power in decision making." In Classics of organization theory, 7th ed. ed. Jay M. Shafritz, J. Steven Ott, and Yong Suk Jang. New York: Wadsworth.

Pierson, Paul. 2000. "Increasing returns, path dependence, and the study of politics." American Political Science Review 94 (June): 251-267.

Popper, Karl. 1959. The logic of scientific discovery. London: Routledge.

Porter, Douglas R., Ben C. Lin, and Richard B. Peiser. 1987. Special districts: A useful technique for financing infrastructure. Washington, D.C.: Urban Land Institute.

Porter, Michael E. 1990. The competitive advantage of nations. New York: Free Press.

Potts, Jason, and Tim Kastelle. 2010. "Public sector innovation research: What's next?" Innovation: Management, policy and practice 12 (2): 122-137.

Putnam, Robert D. 2000. Bowling alone: The collapse and revival of American community. New York: Simon and Schuster.

Quick, Kathryn S., and Martha S. Feldman. 2011. "Distinguishing participation and inclusion." Journal of Planning Education and Research 31(3): 272-90.

Ragin, Charles C. 2014. The comparative method: moving beyond qualitative and quantitative strategies. Berkeley: University of California Press.

Rainey, Hal G. 1997. Understanding and managing public organizations, $2^{\text {nd }}$ ed. San Francisco: Jossey-Bass Publishers.

Ramirez, R. 1999. "Value co-production: Intellectual origins and implications for 
practice and research.” Strategic Management Journal 20 (1): 49-65.

Reese, William L. 1996. Dictionary of philosophy and religion. Amherst: NJ,: Prometheus Books.

Reuvers, Mark, Marloes L. Van Engen, Claartje J. Vinkenburg, and Elisabeth Wilson-Evered. 2008. "Transformational leadership and innovative work behavior: Exploring the relevance of gender differences." Creativity and Innovation Management (n.d.): 227-244.

Reynolds, Paul D. 1971. A Primer in theory construction. New York: Bobbs-Merrill

Roberts, Nancy. 2004. "Public deliberation in an age of direct citizen participation." The American Review of Public Administration 34 (4): 315-353.

Roethlisberger, Fritz J. 1941. "The Hawthrone experiments.” In Classics of organization theory, 7th ed. ed. Jay M. Shafritz, J. Steven Ott, and Yong Suk Jang. New York: Wadsworth.

Rogers, Everett M. 2002. Diffusion of innovation, $5^{\text {th }}$ ed. New York: Free Press.

Rosenfeld, Robert, and Jenny C. Servo. 1990. "Facilitating innovation in large organizations." In Innovation and creativity at work. ed. Michael A. West and James L. Farr. Chichester, UK: John Wiley and Sons.

Rowe, Lloyd A., ed. 1973. Organizational and managerial innovation: a Reader. Pacific Palisades, CA: Goodyear Publishing Company, Inc.

Sahni, Nikhil R., Maxwell Wessel, and Clayton M. Christensen. 2013. "Unleashing breakthrough innovation in government." Stanford Social Innovation Review (Summer): 27-31.

Salamon, Lester M. 2003. The resilient sector. Washington, DC: Brookings Institute Press.

Salge, Torsten O. 2011. "A behavioral model of innovation search: Evidence from public hospital services." Journal of Public Administration Research and Theory (January): $181-210$

Salge, Torstern, and Antonio Vera. 2012. "Benefitting from public sector innovation: The moderating role of customer and learning orientation." Public Administration Review 72 (4): 550-560.

Schein, Edgar H. 2004. “Organizational culture and leadership.” In Classics of organization theory, 7th ed. ed. Jay M. Shafritz, J. Steven Ott, and Yong Suk 
Jang. New York: Wadsworth.

Scott, Richard W. 2003. Organizations: Rational, natural, and open systems. $5^{\text {th }}$ ed. Upper Saddle River, NJ.: Prentice Hall.

Scott, Susanne G., and Reginald A. Bruce. 1994. "Determinants of innovative behavior: A path model of individual innovation in the workplace." The Academy of Management Journal (June): 580-607.

Scutelnicu, Gina, and Sukumar Ganapati. 2012. "Community development districts: An innovative institutional framework for financing and managing infrastructure in Florida." Economic Development Quarterly. 26, 361-372

Sen, Amartya. 1977. "Rational fools: a critique of the behavioral foundations of economic theory." Philosophy and Public Affairs 6 (Summer): 317-344.

Senge, Peter M. 2006. The fifth discipline: the art and practice of the learning organization. New York: Doubleday.

Shafritz, Jay M., Steven Ott, and Yong Suk Jang. 2011. Classics of organizational theory. New York: Wadsworth.

Shin, Janseob, and George E. McClomb. 1998. "Top executive leadership and organizational innovation: an empirical investigation of nonprofit human services organizations (HSO's)." Administration in Social Work 22 (n.d.): 1-21.

Shove, Elizabeth, Mika Pantzar, and Matt Watson. 2012. The dynamics of social practice: Everyday life and how it changes. Thousand Oaks: Sage

Singleton, Royce A. Jr., and Bruce C. Straits. 2010. Approaches to social research, $5^{\text {th }}$ Ed. New York: Oxford University Press.

Smith, Martin J. 2011. "Tsars, leadership and innovation in the public sector." Policy and Politics (n.d.): 343-359.

Sorensen, Eva, and Jacob Torfing. 2012. "Enhancing collaborative innovation in the public sector." Administration and Society (January): 842-868.

Special District Insurance Services Underwriting Department [SADO]. (2013, October 1). 2013. Best practices self-assessment checklist instructions [Memorandum]. Salem, OR: Special District Insurance Services.

Srivastava, Nivedita, and Shreekumar K. Nair. 2010. "Emotional intelligence and managerial effectiveness: role of rational emotive behavior." Indian Journal of Industrial Relations (October): 313-327. 
Stack, Martin and Myles Gartland. 2003. "Path Creation, Path Dependency, and Alternative Theories of the Firm. Journal of Economic Issues (2): 487-494.

Stein, Beverly. 2001. “Start with a blank sheet...” Public Management (April): 21-22.

Stiehm, Judith Hicks, and Nicholas W. Townsend. 2002. The US Army War College: Military education in a democracy. Philadelphia, PA: Temple University Press.

Szkuta, Katarzyna, Roberto Pizzicannella, and David Osimo. 2014. "Collaborative approaches to public sector innovation: A scoping study." Telecommunications Policy (38) (May): 558-567.

Thomas, Gary. 2011. Case study. Thousand Oaks, CA.: Sage Publishing.

Thompson, Victor A. 1973. "Bureaucracy and innovation." In Organizational and managerial innovation: a Reader. ed. Lloyd A. Rowe. Pacific Palisades, CA: Goodyear Publishing Company, Inc.

Tichy, Noel M. and Mary Anne Devanna. 1990. The transformational leader. New York: John Wiley and Sons.

Tushman, Michael, and Charles A O'Reilly. 1997. "Winning through innovation: a practical guide to leading organizational change and renewal." Boston: Harvard Business School Press.

Underwriting Department [SADO]. (2013, October 1). 2013 Best practices selfassessment checklist instructions [Memorandum]. Salem, OR: Special District Insurance Services.

U.S. Census Bureau [Census]. 2002. 2002 Census of Governments, Vol 1, Number 1, Government Organization, GC02(1)-1. Washington, DC: U.S. Government Printing Office. pp. vii-viii.

Vaccaro, Ingacio G., Justin J.P. Jansen, Frans A.J. Van Den Bosch and Henk W. Volberda. 2012. "Management innovation and leadership: The moderating role of organizational size.” Journal of Management Studies 49 (January): 28-51.

Van de Ven, Andrew H. 1986. "Central problems in the management of innovation." Organization Design (May): 590-607.

Van de Ven, Andrew H., Harold L. Angle, and Marshal Scott Poole. 1989a. Research on the management of innovation. New York: Harper and Row.

Van de Ven, Andrew H., and Marshall Scott Poole. 1989. "Toward a general theory of 
innovation processes." In Research on the Management of Innovation. ed. Andrew H. Van de Ven, Harold L. Angle, and Marshal Scott Poole. New York: Harper and Row.

Van der Voet, Joris. 2014. "The effectiveness and specificity of change management in a public organization: Transformational leadership and a bureaucratic organizational structure.” European Management Journal 32 (n/d): 373-382

Veiseh, Seidmehdi, Esfandyar Mohammadi, Mohsen Pirzadian, and Vahid Sharafi. 2014. "The relation between transformational leadership and organizational culture." Journal of Business Studies Quarterly 5 (n.d.): 113-124.

Walker, Richard M. 2006. "Innovation types and diffusion: An empirical analysis of local government." Public Administration 84 (2): 311-335.

Walker, Richard M. 2008. "An empirical evaluation of innovation types and organizational and environmental characteristics: towards a configuration framework." Journal of public administration research and theory (n.d.): 591-615.

Walker, Richard M. 2010. "Innovation." In Public service improvement: theories and evidence. ed. Rachel Ashworth, George Boyne and Tom Entwistle. Oxford: Oxford University Press.

Walker, Richard M. 2013. "Internal and external antecedents of process innovation: A review and extension." Public Management Review 16 (1): 21-44.

Walker, Richard M., Fariborz Damanpour, and Carlos A. Devece. 2011. "Management innovation and organizational performance: The mediating effect of performance management." Journal of Public Administration Research and Theory 21 (April): 367-386.

Wallerstein, Immanuel. 1974. "The rise and future demise of the world capitalist system concepts for comparative analysis." Comparative studies in society and history 16 (September): 387-415.

Walsh, Annmarie Hauck. 1978. The public's business: The politics and practices of government corporations. Cambridge, MA: The MIT Press.

Weber, Max. 1964. "Bureaucracy." In Classics of organization theory, 7th ed. ed. Jay M. Shafritz, J. Steven Ott, and Yong Suk Jang. New York: Wadsworth.

Weerawardena, Jay. 2003. "The role of marketing capability in innovation-based competitive strategy." Journal of Strategic Marketing (n.d.): 15-35. 
West, Michael A. 2002. "Sparkling fountains or stagnant ponds: An integrative model of creativity and innovation implementation in work groups." Applied psychology: An international review (July): 355-387.

West, Michael A., and James L. Farr. 1990. Innovation and creativity at work. Chichester, UK: John Wiley and Sons.

West, Michael A., Carol S. Borrill, Jeremy F. Dawson, Felix Brodbeck, David A. Shapiro, and Bob Haward. 2003. "Leadership clarity and team innovation in health care.” The Leadership Quarterly (n.d.) 393-410.

Whitaker, Gordon P. 1980. "Coproduction: citizen participation in service delivery." Public Administration Review 40 (3): 240-246.

Wright, Bradley E. and Sanjay K. Pandey. 2009. "Transformational leadership in the public sector: Does structure matter?" Journal of Public Administration Research and Theory 20 (April): 75-89.

Yin, Robert K. 1994. Case study Research: Design and methods. $2^{\text {nd }}$ Ed. Thousand Oaks, CA.: Sage Publishing.

Zaltman, Gerald, Robert Duncan, and Jonny Holbek. 1973. Innovations and organizations. New York: John Wiley and Sons.

Zimmermann, Horst. 1999. "Innovation in nonprofit organizations." Annals of Public and Cooperative Economics (n.d.): 589-619. 


\section{Appendix A: Data Collection Instrument (Interview Guide)}

Introduction: Hello [Subject's Name], my name is [Interviewer's Name] and I am a doctoral student at Portland State University. I am conducting research on innovation in Ports in Oregon and Washington.

Informed Consent: I would like to take a few minutes to briefly explain the interview process and get your consent before we proceed with any questions.

[Review the Informed Consent Form with the participant; obtain signature on Form from the participant; and provide a copy of the Form to the participant before proceeding]

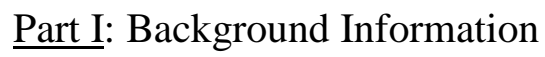

I am going to start by asking you some basic questions about your background and professional work role as it relates to innovation in ports

1) What is your official job title?

2) What role do you play in [name of organization]?

3) What is your professional background?

\section{Part II: Your Story}

* Are there any stories that exemplify organizational innovation or [insert organization's name] role in that process that you are willing to share?

Response:

Part III: Leader-Client Co-production

Keeping in mind your story, I'm going to ask you some open-ended questions and statements about how the Port leadership and their customers and clients interact

Questions related to adaptive interest alignment

1) Did leaders responsible for major innovations receive continuous and real time performance information (e.g., resilience, quality, accessibility, and/or productivity) related to those innovations? (existence of feedback loops)

a) Response:

b) Probe:

2) Did your organization have procedures for securing buy-in from externally impacted customers and clients for legacy processes and technologies when innovations are 
being considered and implemented? (ability to sunset obsolescent technology and processes)
a) Response:
b) Probe:

3) Did your organization's policies (including the vision, mission, values, and strategies) explicitly value organizational innovation? (leading with a vision)
a) Response:
b) Probe:

Part IV: Client-Organization Co-production

Keeping in mind your story, I'm going to ask you some open-ended questions and statements about how the Port as an organization and its customers and clients interact

Questions related to client-based prioritization

1) Were specific demands of customers or clients for innovation clearly reflected in organizational budget priorities? (existence of resource constraints)
a) Response:
b) Probe:

2) Did your organization actively co-produce operational budgets that embrace needed public service innovations with customers and clients? (co-production awareness gaps)
a) Response:
b) Probe:

Questions related to co-production readiness

1) Did your organization examine and effectively phase out a current processes, technology, or human resource in relation to a proposed innovation? (sunset through co-production)
a) Response:
b) Probe: 
2) Did your organization give operational priority to communicating the benefits/value of your innovations with internal and external customers and clients? (information symmetry)
a) Response:
b) Probe:

\section{Part V: Organizational-Leader Co-production}

Keeping in mind your story, I'm going to ask you some open-ended questions and statements about how the Port as an organization and its leadership interact

Questions related to organization's structure/culture

1) Was there latitude to provide leadership to your team in whatever way best suited or motivated them to achieve their goals? (transformational leadership)
a) Response:
b) Probe:

2) Did your organization encourage experimentation and pilot efforts that are "out of the box" in order to foster resilience, quality, accessibility, and/or productivity? (ability to experiment)
a) Response:
b) Probe:

Questions related to organizational incentives

1) Did your organization make consistent and effective uses of both intrinsic (e.g. inspirational motivation, individual autonomy, intellectual stimulation, etc...) and extrinsic (e.g. contingent financial rewards, etc....) incentives related to innovation? (incentives for improvement activity)
a) Response:
b) Probe:

Questions related to risk-taking preferences

1) Did your organization explicitly give priority to risk-taking in its policies, procedures, and everyday practices? (individual risk-taking preference)

a) Response: 
b) Probe:

Part VI: Conclusion

Thank you for your participation. Is there anything you would like to add?

\section{Potential Probe Questions:}

* Do you have the freedom to determine how best to balance the organization's shortterm and long-term needs?

* Do you and individuals in your organization manifest an entrepreneurial spirit by embracing responsible risk-taking in yourself and others?

* How do you help to gain agreement with others on goals and objectives?

* Do you have the freedom to set ambitious targets for yourself that redefine your job?

How do you work with others to ensure that everyone has a stake in success?

* Do your organization's policies and values support an open learning culture that embraces generative learning from both successes and failures?

How do you work with others to coordinate the various components of innovation? 


\section{Appendix B: Case Content Analysis Coding Book (Codebook)}

\begin{tabular}{|c|c|c|c|c|c|}
\hline \multirow{2}{*}{$\begin{array}{l}\text { Domain } \\
\text { (Core } \\
\text { Theoretical } \\
\text { Constraint) }\end{array}$} & \multirow{2}{*}{$\begin{array}{l}\text { Antecedent } \\
\text { (Concept) }\end{array}$} & \multirow[t]{2}{*}{ Indicator } & \multicolumn{3}{|c|}{ Measurement } \\
\hline & & & $\begin{array}{c}\text { Independent Variable (Theme } \\
\text { / Working Definition / } \\
\text { Decision Level) i.e. Evidence } \\
\text { of... } \\
\end{array}$ & $\begin{array}{l}\text { Ordinal } \\
\text { Ranking } \\
\text { Scale }\end{array}$ & Value \\
\hline \multirow[t]{6}{*}{$\begin{array}{l}\text { Leader-Client } \\
\text { Co-Production }\end{array}$} & \multirow[t]{6}{*}{$\begin{array}{l}\text { Adaptive } \\
\text { Interest } \\
\text { Alignment }\end{array}$} & \multirow[t]{3}{*}{$\begin{array}{l}\text { Existence of } \\
\text { feedback } \\
\text { loops }\end{array}$} & $\begin{array}{l}\text { Routinized communications } \\
\text { regarding innovation process } \\
\text { and projects (i.e. regular } \\
\text { meetings, reports, } \\
\text { correspondences, etc.) }\end{array}$ & High & +1 \\
\hline & & & $\begin{array}{l}\text { Random or haphazard } \\
\text { communications regarding } \\
\text { innovation process or projects } \\
\text { (i.e. sporadic meetings, } \\
\text { reports, correspondences, etc.) }\end{array}$ & Medium & 0 \\
\hline & & & $\begin{array}{l}\text { No communications regarding } \\
\text { innovation (i.e. no regular } \\
\text { meetings, reports, } \\
\text { correspondences, etc.) }\end{array}$ & Low & -1 \\
\hline & & \multirow[t]{3}{*}{$\begin{array}{l}\text { Lead with a } \\
\text { vision }\end{array}$} & $\begin{array}{l}\text { Ability to articulate innovation } \\
\text { in relation to institution's } \\
\text { vision or mission statement } \\
\text { (i.e. innovation mentioned in } \\
\text { vision/mission statement) }\end{array}$ & High & +1 \\
\hline & & & $\begin{array}{l}\text { Innovation not directly linked } \\
\text { to institutional vision or } \\
\text { mission statement. }\end{array}$ & Medium & 0 \\
\hline & & & $\begin{array}{l}\text { Not able to articulate } \\
\text { innovation in relation to } \\
\text { institutional vision or mission } \\
\text { statement }\end{array}$ & Low & -1 \\
\hline \multirow[t]{3}{*}{$\begin{array}{l}\text { Client- } \\
\text { Organization } \\
\text { Co-Production }\end{array}$} & \multirow[t]{3}{*}{$\begin{array}{l}\text { Client-Based } \\
\text { Prioritization }\end{array}$} & \multirow[t]{3}{*}{$\begin{array}{l}\text { Existence of } \\
\text { resource } \\
\text { constraints }\end{array}$} & $\begin{array}{l}\text { Evidence of specific demands } \\
\text { of customers or clients for } \\
\text { innovation clearly reflected in } \\
\text { organizational budget priorities }\end{array}$ & High & +1 \\
\hline & & & $\begin{array}{l}\text { Little evidence of customer or } \\
\text { client demands for innovation } \\
\text { given budget priorities }\end{array}$ & Medium & 0 \\
\hline & & & $\begin{array}{l}\text { No evidence of specific } \\
\text { demands of customers or } \\
\text { clients for innovation clearly } \\
\text { reflected in organizational } \\
\text { budget priorities }\end{array}$ & Low & -1 \\
\hline
\end{tabular}




\begin{tabular}{|c|c|c|c|c|c|}
\hline \multirow[t]{9}{*}{$\begin{array}{l}\text { Client- } \\
\text { Organization } \\
\text { Co-Production } \\
\text { (Continued) }\end{array}$} & \multirow[t]{3}{*}{$\begin{array}{l}\text { Client-Based } \\
\text { Prioritization } \\
\text { (Continued) }\end{array}$} & \multirow[t]{3}{*}{$\begin{array}{l}\text { Co- } \\
\text { production } \\
\text { gap } \\
\text { awareness }\end{array}$} & $\begin{array}{l}\text { Evidence organization actively } \\
\text { co-produced operational } \\
\text { budgets that embraced needed } \\
\text { public service innovations with } \\
\text { customers and clients }\end{array}$ & High & +1 \\
\hline & & & $\begin{array}{l}\text { Some evidence organization } \\
\text { actively co-produced } \\
\text { operational budgets with } \\
\text { customers and clients }\end{array}$ & Medium & 0 \\
\hline & & & $\begin{array}{l}\text { No evidence organization } \\
\text { actively co-produced } \\
\text { operational budgets that } \\
\text { embraced needed public } \\
\text { service innovations with } \\
\text { customers and clients }\end{array}$ & Low & -1 \\
\hline & \multirow[t]{6}{*}{$\begin{array}{l}\text { Co-Production } \\
\text { Readiness }\end{array}$} & \multirow[t]{3}{*}{$\begin{array}{l}\text { Sunset } \\
\text { through co- } \\
\text { production }\end{array}$} & $\begin{array}{l}\text { Evidence of systematically } \\
\text { examining and phasing out } \\
\text { current processes, technology } \\
\text { or human resources in relation } \\
\text { to innovation }\end{array}$ & High & +1 \\
\hline & & & $\begin{array}{l}\text { Evidence of minimal efforts to } \\
\text { examine or phase out current } \\
\text { processes, technology, or } \\
\text { human resources in relation to } \\
\text { innovation }\end{array}$ & Medium & 0 \\
\hline & & & $\begin{array}{l}\text { No evidence of examining and } \\
\text { phasing out current processes, } \\
\text { technology, or human } \\
\text { resources in relation to } \\
\text { innovation }\end{array}$ & Low & -1 \\
\hline & & \multirow[t]{3}{*}{$\begin{array}{l}\text { Information } \\
\text { symmetry }\end{array}$} & $\begin{array}{l}\text { Routinized information sharing } \\
\text { and resources dedication to } \\
\text { information sharing (i.e. } \\
\text { priority communicating with } \\
\text { internal and external } \\
\text { customers to share innovation } \\
\text { benefits and value) }\end{array}$ & High & +1 \\
\hline & & & $\begin{array}{l}\text { Random or haphazard sharing } \\
\text { of information and limited } \\
\text { organizational resources } \\
\text { dedicated to information } \\
\text { sharing or articulating benefits } \\
\text { and value to customers }\end{array}$ & Medium & 0 \\
\hline & & & $\begin{array}{l}\text { No communications regarding } \\
\text { innovation (i.e. no regular } \\
\text { meetings, reports, } \\
\text { correspondences, etc.) }\end{array}$ & Low & -1 \\
\hline
\end{tabular}




\begin{tabular}{|c|c|c|c|c|c|}
\hline \multirow[t]{12}{*}{$\begin{array}{l}\text { Organizational } \\
\text {-Leader Co- } \\
\text { Production }\end{array}$} & \multirow[t]{6}{*}{$\begin{array}{l}\text { Organizational } \\
\text { Structure / } \\
\text { Culture }\end{array}$} & \multirow[t]{3}{*}{$\begin{array}{l}\text { Transform- } \\
\text { ational } \\
\text { leadership }\end{array}$} & $\begin{array}{l}\text { Identifies three or more } \\
\text { characteristics of } \\
\text { transformational leadership } \\
\text { (Bass 1985): } \\
\text { a. Idealized Influence } \\
\text { b. Inspirational Motivation } \\
\text { c. Intellectual Stimulation } \\
\text { d. Individualized Consideration }\end{array}$ & High & +1 \\
\hline & & & $\begin{array}{l}\text { Identifies mix of three or more } \\
\text { characteristics of } \\
\text { transformational and } \\
\text { transactional leadership } \\
\text { (Bass 1985) }\end{array}$ & Medium & 0 \\
\hline & & & $\begin{array}{l}\text { Identifies three or more } \\
\text { characteristics of transactional } \\
\text { leadership (i.e. own-interest, } \\
\text { rewards focus, etc.) (Bass } \\
\text { 1985) }\end{array}$ & Low & -1 \\
\hline & & \multirow[t]{3}{*}{$\begin{array}{l}\text { Ability to } \\
\text { experiment }\end{array}$} & $\begin{array}{l}\text { Evidence of three or more } \\
\text { systemic, time-phased } \\
\text { experiments tied to one or } \\
\text { more innovation initiative }\end{array}$ & High & +1 \\
\hline & & & $\begin{array}{l}\text { Evidence of non-systemic or } \\
\text { random experimentation not } \\
\text { tied to specific innovation } \\
\text { initiative }\end{array}$ & Medium & 0 \\
\hline & & & $\begin{array}{l}\text { No evidence of } \\
\text { experimentation exists }\end{array}$ & Low & -1 \\
\hline & \multirow[t]{3}{*}{$\begin{array}{l}\text { Organizational } \\
\text { Incentives }\end{array}$} & \multirow{3}{*}{$\begin{array}{l}\text { Incentives } \\
\text { for improve- } \\
\text { ment } \\
\text { activity }\end{array}$} & $\begin{array}{l}\text { Both intrinsic AND extrinsic } \\
\text { incentives linked to supporting } \\
\text { or participating in innovation }\end{array}$ & High & +1 \\
\hline & & & $\begin{array}{l}\text { Evidence of EITHER intrinsic OR } \\
\text { extrinsic incentives linked to } \\
\text { innovation (but not both) }\end{array}$ & Medium & 0 \\
\hline & & & $\begin{array}{l}\text { No evidence of intrinsic OR } \\
\text { extrinsic incentives }\end{array}$ & Low & -1 \\
\hline & \multirow[t]{3}{*}{$\begin{array}{l}\text { Risk-taking } \\
\text { Preferences }\end{array}$} & \multirow[t]{3}{*}{$\begin{array}{l}\text { Individual } \\
\text { risk-taking } \\
\text { preference }\end{array}$} & $\begin{array}{l}\text { Expressed willingness to take } \\
\text { risks when reward is equal or } \\
\text { greater than the risk involved }\end{array}$ & High & +1 \\
\hline & & & $\begin{array}{l}\text { Expressed willing to take some } \\
\text { risks, but significant reward } \\
\text { must result and risk is } \\
\text { mitigated }\end{array}$ & Medium & 0 \\
\hline & & & $\begin{array}{l}\text { Expressed desire to avoid risk } \\
\text { or statements of extensive risk } \\
\text { mitigation activity around } \\
\text { innovation }\end{array}$ & Low & -1 \\
\hline
\end{tabular}

